\title{
THE VERTICAL STRUCTURE OF THE BOTTOM BOUNDARY LAYER ON THE SOUTHERN FLANK OF GEORGES BANK \\ DURING LATE WINTER
}

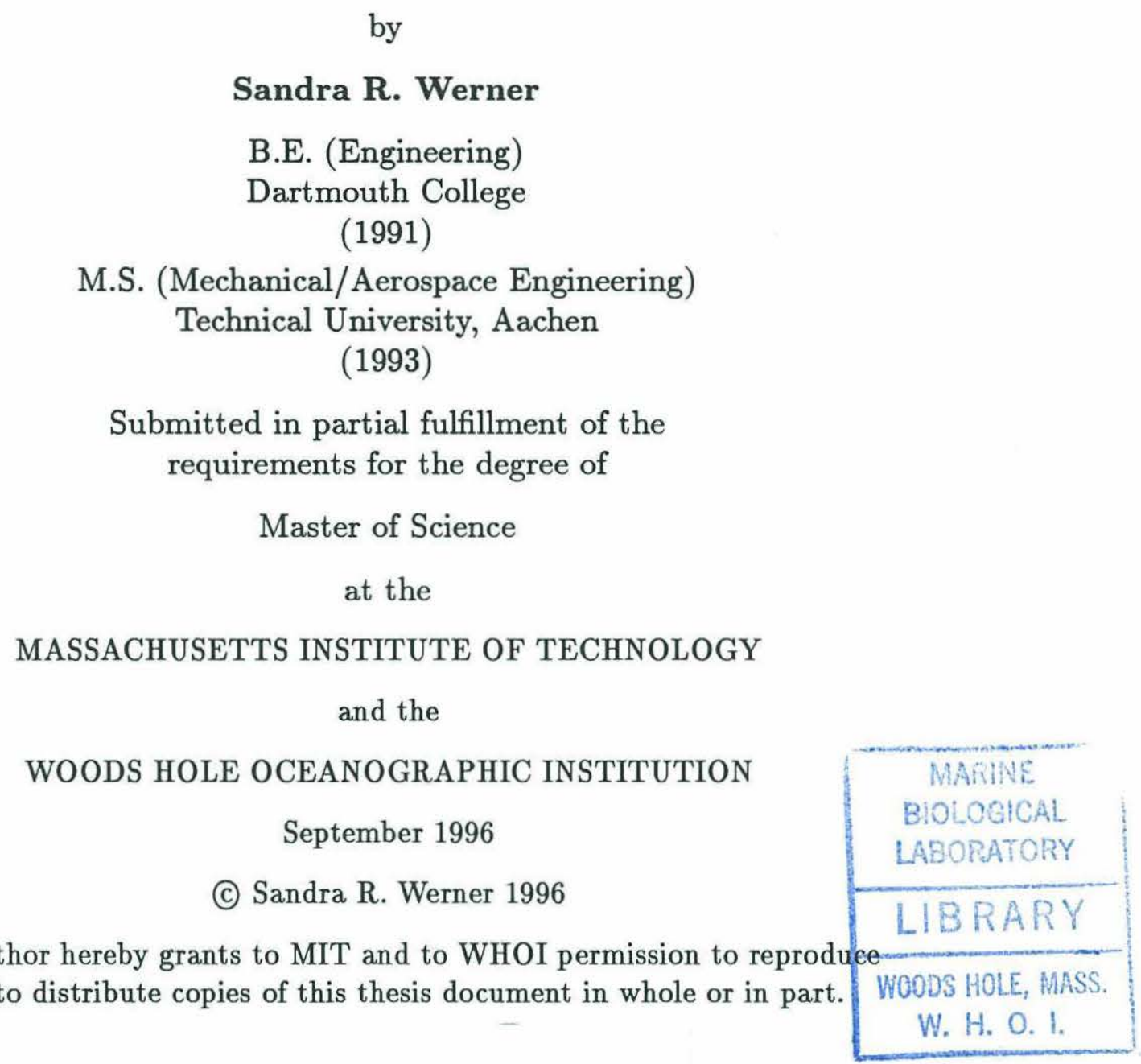

Signature of Author .

Joint Program in Physical Oceanography

Massachusetts Institute of Technology

Woods Hole Oceanographic Institution

August 29, 1996

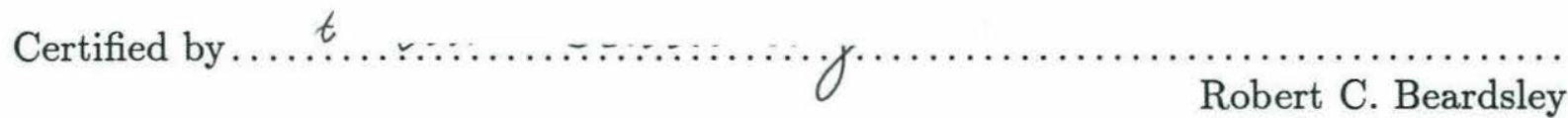

Senior Scientist

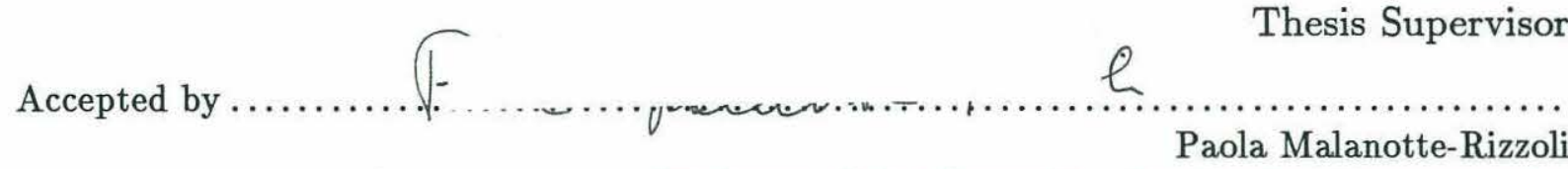

Chairman, Joint Committee for Physical Oceanography

Massachusetts Institute of Technology

Woods Hole Oceanographic Institution 


\title{
The Vertical Structure of the Bottom Boundary Layer on the Southern Flank of Georges Bank During Late Winter
}

\author{
by
}

Sandra R. Werner

Submitted in partial fulfillment of the requirements for the degree of Master of Science at the Massachusetts Institute of Technology and the Woods Hole Oceanographic Institution

\begin{abstract}
The bottom boundary layer structure on the southern flank of Georges Bank was investigated for the period February 19 to March 20, 1995. During this time, vertical stratification was weak and the near bottom waters were almost homogeneous. Georges Bank is a region of strong tidal currents, with the dominant constituent, the $M_{2}$, carrying most of the tidal energy. A clockwise around-bank circulation is present throughout the year, with flow directions being to the southwest along the southern flank, and to the northeast along the northern flank. Main generation mechanisms of the mean circulation are tidal rectification over the sloping bottom, horizontal stratification, and, along the continental shelf, flow associated with upstream sources including the Labrador Current. Due to the effects of winter cooling, cross-bank density gradients are weak during winter, and the clockwise mean flow is smallest during the cold seasons.

Velocity, temperature, and salinity data were taken at Stratification Site $1\left(40^{\circ} 51^{\prime} \mathrm{N}\right.$, $67^{\circ} 33^{\prime} \mathrm{W}$ ) located on the $76 \mathrm{~m}$ isobath $30 \mathrm{~km}$ upslope of the shelf slope front. The $M_{2}$ pressure gradient was almost rectilinear and across-bank, forcing a current ellipse with eccentricity $e \approx 0.62$ and depth-averaged current amplitudes of $\approx 40 \mathrm{~cm} / \mathrm{s}$. The magnitude of the depth-averaged mean flow is $9 \pm 5 \mathrm{~cm} / \mathrm{s}$ for the period investigated and small compared to the tidal currents. Estimates of friction velocities and bottom roughness were obtained from best-fit logarithmic profiles to velocity measurements taken between 0.28 and $2.5 \mathrm{~m}$ above the sea floor.

Boundary layer heights for the tidal and subtidal flows were derived from profiles of the $M_{2}$ and low-pass filtered currents. Meaningful parameterizations of these heights were found from the equations of motion assuming a logarithmic velocity region in the vicinity of the sea floor as suggested by the observations. Scaling arguments show that the mean and tidal flows interact through the effects of bottom friction, with the largest part of the turbulent fluctuations being set by the $M_{2}$ tide. Scaling arguments
\end{abstract}


for the tidal boundary layer height were discussed with respect to earlier results by Soulsby (1990). Integrated velocity defects were computed for the $M_{2}$ and low-pass filtered flow, and compared to boundary layer transports predicted by the estimated bottom stress. Closer investigation of subtidal velocity defects reveals the importance of advective terms in the time-averaged along-bank momentum equation, in agreement with previous studies (Zimmermann, 1980; Loder, 1980; Huthnance, 1981) discussing the nonlinear character of tidal rectification.

In a numerical modeling study, the performance of a simple one-dimensional, two-layer model was examined. Numerical mixing coefficients were parameterized according to $K=\kappa \bar{u}_{*} z$ in the sublayer $z \leq l$, and $K=\kappa \bar{u}_{*} l$ in the rest of the water column, where $\bar{u}_{*}$ is the mean friction velocity during one tidal cycle. Based on the comparison of model solutions to observations, a characteristic parameterization for the sublayer thickness was derived, suggesting optimal values for $l$ to be similar to the observed logarithmic layer height $l \approx 5 \mathrm{~m}$. Numerical predictions using the Mellor-Yamada level 2.5 turbulence closure were also investigated. The performance of this advanced closure scheme was found to be less convincing than results from the much simpler two-layer model.

Thesis Advisor:

Robert C. Beardsley, Senior Scientist

Physical Oceanography Department

Woods Hole Oceanographic Institution 


\section{Table of Contents}

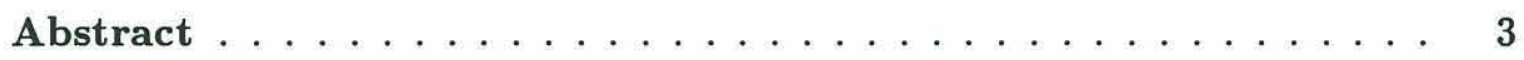

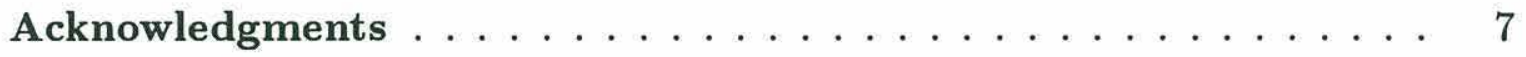

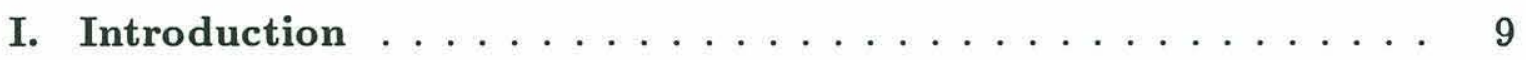

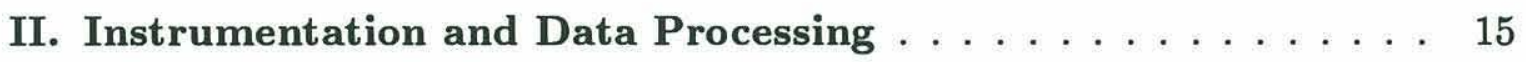

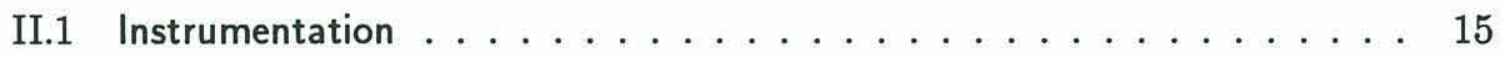

II.1.1 Mooring Site . . . . . . . . . . . . . . . . 15

II.1.2 Moored Array . . . . . . . . . . . . . . . . . 15

II.1.3 Surface and Subsurface Mooring . . . . . . . . . . . . . 16

II.1.4 Benthic Acoustic Stress Sensor (BASS) Tripod . . . . . . . . . 17

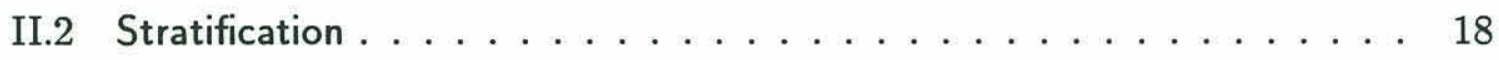

II.3 Processing BASS Velocity Data . . . . . . . . . . . . . . . . 22

II.4 Estimating Bottom Stress . . . . . . . . . . . . . . . . . . 25

II.4.1 Friction Velocity . . . . . . . . . . . . . . 25

II.4.2 Bottom Roughness $\ldots \ldots \ldots \ldots \ldots$

II.4.3 Bottom Stress Vector . . . . . . . . . . . . . . . . . 29

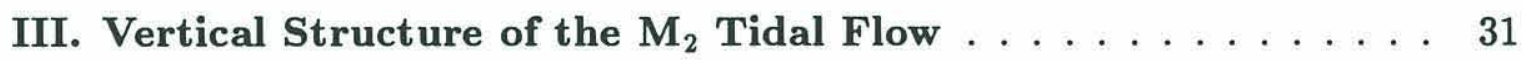

III.1 Introduction . . . . . . . . . . . . . . . . . . . . 31

III.2 Data Analysis . . . . . . . . . . . . . . . . . . . . . . 31

III.2.1 Tidal Current Analysis . . . . . . . . . . . . . . . . . 31

III.2.2 The $\mathrm{M}_{2}$ Current Ellipse $\ldots \ldots \ldots \ldots \ldots \ldots \ldots$

III.2.3 Boundary Layer Height Scaling . . . . . . . . . . . . . 36

III.2.4 The $\mathrm{M}_{2}$ Stress Ellipse $\ldots \ldots \ldots \ldots \ldots$. . . . . . . . 41

III.2.5 Boundary Layer Transports . . . . . . . . . . . . . . 44

III.2.6 Eddy Viscosity Profiles . . . . . . . . . . . . . . . . . 46 
III.3 Numerical Modeling . . . . . . . . . . . . . . . . . . . . . . 49

III.3.1 Model Description . . . . . . . . . . . . . . . . . . . . 49

III.3.1.1 The $K$-Profile Model . . . . . . . . . . . . . . . . . . 49

III.3.1.2 The Mellor-Yamada Level 2.5 Closure . . . . . . . . . . 52

III.3.2 Model Performance . . . . . . . . . . . . . . . . . . . 54

III.3.2.1 Performance of the $K$-Profile Model $\ldots \ldots \ldots \ldots$

III.3.2.2 Performance of the MY 2.5 . . . . . . . . . . . . . 61

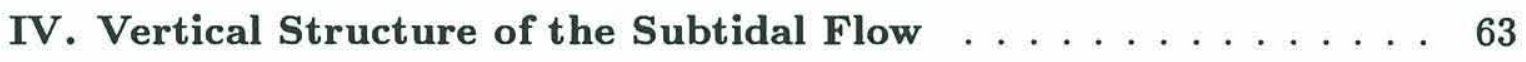

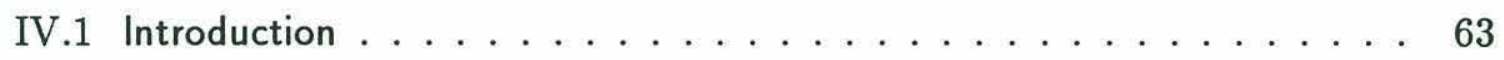

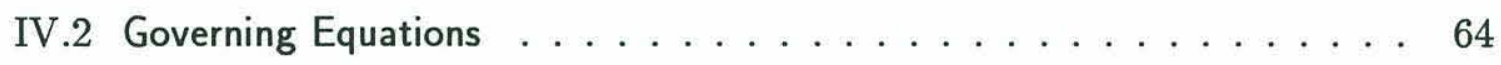

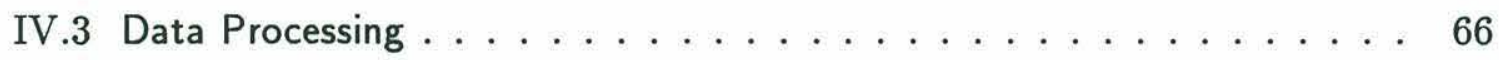

IV.4 Interior Flow Regime $\ldots \ldots \ldots \ldots \ldots \ldots \ldots$

IV.5 Scaling of the Subtidal Boundary Layer Height $\ldots \ldots \ldots$

IV.6 Subtidal Boundary Layer Transports . . . . . . . . . . . . . . 70

V. Summary and Conclusions $\ldots \ldots \ldots \ldots 73$

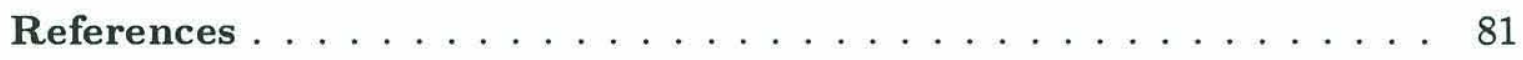

List of Figures $\ldots \ldots \ldots \ldots \ldots \ldots \ldots \ldots$

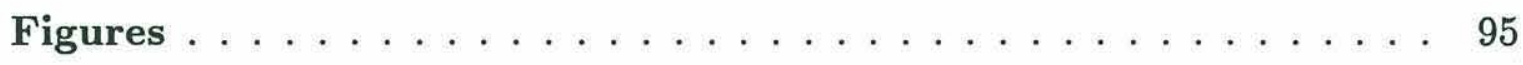




\section{Acknowledgments}

First, I would like to thank my academic advisor, Robert Beardsley, and my coadvisors John Trowbridge and Steve Lentz for their guidance and support throughout this work, and for always making their time available when advice was needed. Helpful discussions with Jim Price and Glen Gawarkiewicz pointed out several interesting aspects which were incorporated in this thesis. Special thanks are also due to Sandy Williams, Jim Churchill, and Tom Gross (Skidaway Institute of Oceanography) for their assistance and experience in analyzing BASS data. The tidal analysis was carried out with the numerical code of M.G.G. Foreman (Institute of Ocean Sciences, Sidney, B.C.), which was kindly provided by Julio Candela. Robert Beardsley provided the numerical version of the Mellor-Yamada level 2.5 turbulence closure scheme used in this study.

Anne-Marie Michael's help was invaluable for bringing this thesis into its final form and keeping the stress-level close to the deadline within bearable limits. Carol Alessi was always available to assist with the data-set as well as computer problems of any kind.

Finally, I would like to thank my fellow Joint-Program students, in particular Melissa Bowen, Deborah Hassler, and Dan Goldner, for their encouragement during the past year. Without their moral support the successful completion of this thesis would have been difficult.

This work was supported by the National Science Foundation under grant OCE 9313671 as part of the U.S. GLOBEC/Georges Bank Program. 


\section{Introduction}

Georges Bank is a shallow area of $\approx 46,000 \mathrm{~km}^{2}$ located between the Gulf of Maine and the continental slope (Figure 1). The bank is a region of strong tides, with the dominant constituent, the $\mathrm{M}_{2}$, carrying most of the tidal energy. In addition, a clockwise mean circulation is present, with current directions being to the southwest along the southern flank, and to the northeast along the northern flank of the bank. Main contributions to the around-bank circulation are thermal wind currents, tidally rectified currents, and upstream sources. Their presence is explained by the following physical processes:

Thermal wind currents are caused by density gradients at the northern and southern flanks of the bank. In summer, the competing effects of friction-induced turbulent mixing and increasing stratification manifest as density fronts near the $50 \mathrm{~m}$ isobath, separating the well-mixed, homogeneous waters on the crest of the bank from the vertically stratified deeper regions. Below the pycnocline, the resulting density gradients are off-bank, thus driving a clockwise mean flow. Since the $\mathrm{M}_{2}$ tide carries most of the kinetic energy during the warmer seasons and the top of the bank is sufficiently shallow, the transition from well-mixed to vertically-stratified regions is commonly refered to as a tidal mixing front. On the southern flank, the clockwise circulation is intensified by thermal wind currents connected to the shelf slope front near the $100 \mathrm{~m}$ isobath (Flagg, 1987, Butman et al., 1987). The shelf slope front marks the transition from relatively cool, fresh shelf water to warm and saline slope water. Salinity outweights the effects of temperature, leading to a density gradient in the off-bank direction. In late fall and winter, decreasing vertical stratification causes the tidal mixing fronts to dissapear and the shelf slope front to weaken. Scaling arguments based on CTD cross-sections reveal that depth-averaged values of thermal wind currents on the southern flank of Georges Bank are $\approx 2 \mathrm{~cm} / \mathrm{s}$ in winter. Seasonal fluctuations are believed to be $\approx 6 \mathrm{~cm} / \mathrm{s}$ (Butman et al., 1986), with largest values occuring in August and September, and smallest values occuring in January and February. 
Tidal rectification is caused by the presence of a bottom slope together with the effects of friction (Huthnance, 1973; Zimmermann, 1980; Loder, 1980; Butman et al., 1986). The tidally rectified flow is along-isobath and clockwise around the bank, thus adding to the thermal wind currents discussed above. Its magnitude and vertical structure are not easily determined, since they depend on the unknown timeaveraged advective terms as well as on the friction coefficient relating the mean current to the bottom stress. Loder's (1980) analytic theory and Chen's (1992) numerical model solutions suggest rectified velocities of order $2-3 \mathrm{~cm} / \mathrm{s}$ near the $75 \mathrm{~m}$ isobath, if the water column is homogeneous. According to Chen (1992), the presence of vertical stratification may intensify the rectified flow, leading to surface velocities that approach $\approx 5 \mathrm{~cm} / \mathrm{s}$ at similar depths for $N^{2} \approx 1 \cdot 10^{-5} \mathrm{~s}^{-2}$.

The main upstream supply of water to the southern flank of Georges Bank is provided by the Labrador Current and the major coastal rivers, especially the St. Laurence River (Chapman et al., 1989). After passing the Grand Banks of Newfoundland, the Labrador Current flows southward along the continental shelf and is at times augmented by intermittend intrusions of Scotian Shelf water (Bisagni, 1996).

Due to the shallowness of Georges Bank, bottom friction has a significant impact on the vertical structure of the tidal and subtidal flow. Friction induced turbulence is sustained by flow shear, which is strongest near the bottom where turbulent fluctuations are large. The vertical extent and structure of the resulting bottom boundary layer are important factors in setting the vertical stratification on the shelf. On the southern flank of Georges Bank, intermittend intrusions of slope water may modify the near bottom waters, leading to augmented or weakened stratification during events of on-bank or off-bank advection, respectively. Such events are associated with shear instabilities in the shelf slope front or the advection of denser slope water up- or down slope by offbank Gulf Stream warm core rings.

At times of large surface winds, a surface mixed layer of considerable depth may develop, partly overlapping with the bottom boundary layer caused by bottom friction. At such times, surface and bottom stresses can be in near balance, resulting in flow transports that are closely aligned with the wind. This behaviour is observed during 
large winter storms, where strong off-bank winds drive significant quantities of water off-bank towards the open ocean.

Since over wide areas of the bank, the $\mathrm{M}_{2}$ currents represent the dominant flow component, they also account for most of the shear in the vicinity of the sea floor. Therefore, the magnitude of the bottom stress is largely determined by the $\mathrm{M}_{2}$ tide, and the bottom boundary layer is mainly tidally driven. However, the turbulent fluctuations near the sea floor affect the subtidal flow as well as the tidal flow, thus causing the $\mathrm{M}_{2}$ currents to interact with the mean around-bank circulation.

In order to obtain a better understanding of the physics involved in setting the vertical stratification on the bank, the subtidal along- and across-bank transports, and the dominant momentum balance at times of strong and weak surface winds, it is essential to gain insight into the physical mechanisms determining vertical mixing. Since bottom friction has a significant impact on the flow structure in shallow regions, a first step was taken by investigating the bottom boundary layer under vertically homogeneous conditions. The investigation focuses on the southern flank of Georges Bank, a region where subtidal flow speeds are small compared to the $\mathrm{M}_{2}$ velocities, except during winter storm events. Depth averaged tidal amplitudes are of order $40 \mathrm{~cm} / \mathrm{s}$ between the 60 and $100 \mathrm{~m}$ isobaths, and are related to a $\mathrm{M}_{2}$ pressure gradient which is almost rectilinear and cross-bank. In comparison, mean flow speeds are characteristically between 5 and $15 \mathrm{~cm} / \mathrm{s}$, if surface winds are moderate.

Previous research on tidally driven boundary layers includes work by Maas and van Haren (1987), and Soulsby (1990). Their studies discuss observations of the bottom boundary layer under vertically homogeneous and stratified conditions. However, none or only limited current measurements were available near the sea floor, making it difficult to derive estimates for the friction velocities and corresponding bottom stresses. Modeling studies of tidal boundary layers frequently implement the effects of friction by making use of advanced turbulence closure schemes based on the turbulent kinetic energy equation and prognostic or diagnostic expressions for the turbulent mixing length (Davies and Jones, 1990; Chen, 1992). The performance of such models is believed to be good, but comparisons of model results to estimated bottom stresses 
and measured data in the lower part of the water column are a widely underexplored topic. More detailed research has been carried out on rectilinear oscillatory flows (Jonsson and Carlsen, 1976; Trowbridge et al., 1984). Trowbridge et al. (1984) used a one-dimensional, two-layer eddy viscosity model to predict the vertical structure of a homogeneous, rectilinear flow, concluding that model predictions agree well with laboratory results from Jonsson and Carlsen (1976).

In this study the vertical structure of the tidal and subtidal bottom boundary layer is investigated for the period from February 19 to March 20, 1995. Velocity, temperature, and salinity data were taken at Stratification Site 1 (ST1), located at $40^{\circ} 51^{\prime} \mathrm{N}, 67^{\circ} 33^{\prime} \mathrm{W}$ on the $76 \mathrm{~m}$ isobath of the southern flank of Georges Bank (Figures 1 and 2). Vertical stratification was weak during the period investigated $\left(\approx 1 \cdot 10^{-5} \mathrm{~s}^{-2}\right)$, and the lowest $30 \mathrm{~m}$ of the water column were nearly homogeneous. The mooring site is approximately $30 \mathrm{~km}$ to the northwest of the shelf slope front, and $20 \mathrm{~km}$ to the southeast of the tidal mixing front. The bottom slope at this location is $\approx 8 \cdot 10^{-4}$. ST1 was part of the U.S. GLOBEC Stratification Study array and consisted of a surface and a subsurface mooring, each supporting several temperature and conductivity sensors as well as vector-measuring current meters. In addition, a tripod was deployed on the seafloor to provide temperature and acoustic current meter measurements. Estimates of friction velocities, $u_{*}$, and bottom roughness, $z_{0}$, were obtained from best-fit logarithmic profiles to benthic acoustic stress sensor (BASS) data taken at heights 0.22 , $0.58,1.18,2.53$, and $4.43 \mathrm{~m}$ above the bottom. VMCM measurements were available throughout most of the water column, allowing the height of the turbulent layer to be deduced from current profiles. Depth-averaged $\mathrm{M}_{2}$ amplitudes were about $40.3 \mathrm{~cm} / \mathrm{s}$ and $25.8 \mathrm{~cm} / \mathrm{s}$ in the cross- and along-bank directions, respectively. Similar to all other locations on the southern flank of Georges Bank, the $\mathrm{M}_{2}$ current ellipse at ST1 rotates clockwise. Depth averaged subtidal velocities were of order $9 \pm 5 \mathrm{~cm} / \mathrm{s}$ and small compared to the semidiurnal tidal flow.

The thesis is divided into the following sections: Chapter II gives an overview about the mooring site, instrumentation, and data processing. Chapter III presents the results from current meter data and describes the vertical structure of the $\mathrm{M}_{2}$ current 
ellipse. The discussion focuses on the scaling of tidal boundary layer heights, computation of tidal stresses and boundary layer transports, and evaluation of vertical mixing coefficients. Observations are compared to results from a one-dimensional numerical model, making use of a two-layer eddy viscosity with $K=\kappa \bar{u}_{*} z$ in the sublayer $z \leq l$, and $K=\kappa \bar{u}_{*} l$ in the rest of the water column. In this parameterization, $K$ is an eddy viscosity, $\bar{u}_{*}$ is the mean friction velocity during one tidal cycle, and $z$ is the height above the bottom. Numerical model results using the Mellor-Yamada level 2.5 turbulence closure are also investigated and compared to results of the much simpler eddy-viscosity model. In chapter IV, the vertical structure of the subtidal flow is discussed, and the importance of nonlinear advective terms in the subtidal momentum balance is examined. Chapter V summarizes the results. 


\section{Instrumentation and Data Processing}

\section{II.1 Instrumentation}

\section{II.1.1 Mooring Site}

The data used in this study were taken at Stratification Site 1 (ST1) located at $40^{\circ} 51^{\prime} \mathrm{N}, 67^{\circ} 33^{\prime} \mathrm{W}$ on the southern flank of Georges Bank. The water depth at ST1 is $76 \mathrm{~m}$, and the bottom slope is approximately $8 \cdot 10^{-4}$. Bottom sediments are mediumto-coarse sand, with median grain size of 0.25 to $1 \mathrm{~mm}$. ST1 is approximately $30 \mathrm{~km}$ to the northwest of the shelf slope front, and $20 \mathrm{~km}$ to the southeast of the tidal mixing front. The water column is mostly homogeneous during late fall and winter, but vertical and horizontal stratification are present during spring and summer. Depth averaged values of the $M_{2}$ current ellipse are $40.3 \mathrm{~cm} / \mathrm{s}$ and $25.8 \mathrm{~cm} / \mathrm{s}$ for the major and minor axes, respectively. The inclination of the major axis is approximately $62^{\circ}$ clockwise from east and thus roughly across isobath. The depth-averaged subtidal flow is of order $9 \pm 5 \mathrm{~cm} / \mathrm{s}$ and small compared to the semidiurnal tidal flow. According to observations at ST1, these values are representative of summer as well as winter subtidal flows, as long as major storm events are excluded.

\section{II.1.2 Moored Array}

The mooring array at ST1 consisted of a surface and a subsurface mooring, each supporting several vector measuring current meters (VMCMs), temperature, and conductivity recorders. A Benthic Acoustic Stress Sensor (BASS) tripod is deployed on the seafloor to provide measurements of the near-bottom velocities and temperatures. A schematic picture of the mooring array at ST1 is given in Figure 2.

Both the surface and subsurface moorings were deployed from February 3 to August 23, 1995. Measurements from two successive BASS tripod deployments are available for February 3 to April 4, 1995 (BASS1), and June 11 to August 23, 1995 
(BASS2). In the following, time will be referred to as yearday, where the yearday for January 1, 1995 at noon Greenwich Mean Time (GMT) is taken as 0.5.

\section{II.1.3 Surface and Subsurface Mooring}

The surface mooring was equipped with eight VMCMs at heights $70.85,68.50$, $66.05,62.00,57.00,51.00,45.00$, and $39.00 \mathrm{~m}$ above the bottom, recording velocity and temperature at a sample rate of $1.5 \mathrm{~min}$, four internally recording temperature/conductivity instruments (Seacats) at heights 74.50, 69.75, 64.95 and $49.90 \mathrm{~m}$ above the bottom, recording temperature and conductivity at a sample rate of $1.5 \mathrm{~min}$, six single-channel temperature loggers (TPODS) at heights 63.25, 59.00, 53.50, 47.25, 41.50 , and $35.25 \mathrm{~m}$ above the bottom, recording temperature at a sample rate of $30 \mathrm{~min}$, and one miniature temperature recorder (MTR) at height $72.50 \mathrm{~m}$ above the bottom, recording temperature at a sample rate of $30 \mathrm{~min}$. Wind speed and direction, air temperature, air pressure, relative humidity, long- and shortwave radiation, and water temperature were measured by a vector averaging wind recorder (VAWR) and an Improved Meteorological Recorder (IMET), both mounted on top of the $3 \mathrm{~m}$ discus buoy supporting the subsurface instruments. Wind data were taken approximately $3.5 \mathrm{~m}$ above sea level at sample rates 7.5 and $15 \mathrm{~min}$ for the IMET and the VAWR, respectively.

The subsurface mooring was equipped with five VMCMs at heights 30.50, 24.00, $18.00,12.00$, and $6.00 \mathrm{~m}$ above the bottom, two Seacats at heights 29.40 and $10.90 \mathrm{~m}$ above the bottom, and two TPODs at heights 14.50 and $8.50 \mathrm{~m}$ above the bottom. Sample rates were the same as for the surface mooring. More detailed information on instrumentation can be found in Cruise Report EN260 (1995).

The VMCMs at heights 24.00 and $18.00 \mathrm{~m}$ returned noisy or no velocity and temperature data and were not used in this study. Velocity data taken $51.00 \mathrm{~m}$ above the bottom were also excluded from the analysis due to instrumentation problems. Temperature data from the TPODs at heights 59.00 , and $41.50 \mathrm{~m}$, as well as from the VMCM at height $6.00 \mathrm{~m}$ could not be recovered. 
The VMCM velocity data used in this study are hourly averaged, with centerpoints being on the full hour. It is assumed that measurement errors of both the northward and eastward velocity components may be as large as $\pm 2 \mathrm{~cm} / \mathrm{s}$. The orientation of true north as infered from the compass of each VMCM unit is believed to be correct up to $\pm 5^{\circ}$ (Lentz, personal communication).

\section{II.1.4 Benthic Acoustic Stress Sensor (BASS) Tripod}

The BASS tripod supports eight thermistors at heights $0.24,0.62,1.22,1.90$, $2.53,3.24,4.43$, and $5.72 \mathrm{~m}$ above the bottom (thermistors 1-8), five optical backscatter sensors (OBS) at heights $0.11,0.67,1.27,2.45$, and $4.45 \mathrm{~m}$ above the bottom, and five acoustic current meters (benthic acoustic stress sensors) at heights $0.24,0.60,1.20$, 2.55 , and $4.45 \mathrm{~m}$ above the deck (pods 1-5). It wass assumed that upon deployment on the ocean floor, the tripod sank into the ground by approximately $2 \mathrm{~cm}$, yielding instrumentation heights above the sea floor that are $2 \mathrm{~cm}$ below the above-deck values given above. A schematic drawing of the tripod is given in Figure 3. Detailed information about the geometry and function of the benthic acoustic stress sensors can be found in Williams et al. (1987). Although the term BASS applies to the acoustic current meters, it will also be used in the following to refer to the entire tripod and its instrumentation.

Temperature, OBS, and velocity data were recorded at a sample rate of $2 \mathrm{~Hz}$ during bursts of 7.5-min length. One burst occurs every half hour, with centerpoints being 3.25 min after the half and the full hour. In this study, only burst-averaged data will be analyzed.

As indicated in Figure 3, the first BASS tripod (BASS1) was equipped with a camera taking one picture of the seafloor every eight hours. No camera is mounted on the second tripod (BASS2). Although photographs of the seafloor may reveal important information about bottom topography, sand ripples, and sediment resuspension, the camera and light strobe must be expected to obstruct the flow to some degree and affect the velocity measurements. 
In the course of this study, only data from the BASS1 deployment have been analyzed. Data were recovered from all instruments mounted on BASS1, with the exception of thermistor 1. The possible error of the tripod compass was $\pm 15^{\circ}$. The precise measurement error of the acoustic current sensors is not known, but is expected to be significantly smaller than that of the VMCMs in the absence of flow distortion (Lentz et al., 1995). For the purpose of error analysis of the burst-averaged velocity data, the standard deviation within each burst will be used as a representative uncertainty.

\section{II.2 Stratification}

Since the purpose of this study is to examine the bottom boundary layer structure under homogeneous conditions, a time period needs to be found where the lower part of the water column is unstratified. Unfortunately, no salinity data are available over the BASS height, and conclusions about the homogeneity of the near-bottom waters must be drawn from temperature data alone. The accuracy of the tripod thermistors is $\pm 0.001^{\circ} \mathrm{C}$ and lies within the noise level of the calibration, which is about $\pm 0.01^{\circ} \mathrm{C}$. Since during late winter, temperature differences over the bottom $6 \mathrm{~m}$ are generally less than $0.005^{\circ} \mathrm{C}$, it is difficult to obtain quantitative statements about the vertical temperature distribution near the seafloor. An attempt to correct the measurements for the calibration error of the instruments was made by choosing a time period when temperature is believed to be homogeneous over the tripod height, and by adjusting all BASS thermistors such that their mean value during this period equals the mean value measured at thermistor 2 . Figure 4 displays the temperature difference between thermistors 8 and 2 at 5.72 and $0.62 \mathrm{~m}$ above the bottom as a function of time after the adjustment. Mean temperatures over the entire time period were around $5.38^{\circ} \mathrm{C}$. The time period chosen for the adjustment was yearday 62 to 70 .

It can be seen from Figure 4 that events of large thermal stratification occur at different times during the BASS1 deployment. For example, warm water overlies cold water between yearday 37 and 42 , and on yearday 122, while cold water overlies warm water between yeardays 92 and 98 . During these times, buoyancy frequencies squared 
computed from temperature differences, but ignoring vertical changes in salinity, are of order $(1-2) \cdot 10^{-5} \mathrm{~s}^{-2}$ over the tripod height, and they are negative if temperatures decrease upward. Events of significant thermal stratification near the seafloor might be explained by the fact that the shelf slope front to the south east of ST1 is not stationary, and moves at times upslope or downslope. Such motion could for example be caused by strong surface winds, or advection due to Gulf Stream rings. Since velocities are strongly sheared near the bottom, on- or offbank motion of slope water may account for the vertical stratification observed in the temperature data. Satellite imagery shows that during the first half of 1995 , several warm core rings moved along the continental slope, advecting warm slope water upslope, or fresh shelf water offshelf. Wind data taken by the IMET during the BASS1 deployment are shown in Figure 5. It can be seen from the figure that wind speeds exceeded $15 \mathrm{~m} / \mathrm{s}$ at all stratification events described above and were large compared to the time-mean value of $7 \mathrm{~m} / \mathrm{s}$. However, there is no apparent relation between the orientation of the wind vector and the occurrence of either positive or negative thermal stratification at these times. This result is to be expected, since surface- and bottom-mixed layers can overlap when wind stresses are large, so that simple Ekman dynamics do not apply and flow directions near the bottom cannot be easily predicted. The vertical flow structure during times of strong winds is complicated and shall not be investigated here.

During the second half of the BASS1 deployment, Figure 4 shows a gradual increase of less than $0.01^{\circ} \mathrm{C}$ in the temperature difference between thermistors 8 and 2 , particularly visible towards the end of the time series. Since similar behavior was found to occur at other BASS thermistors, as well as at the TPODS at 14.5 and $8.5 \mathrm{~m}$ above the bottom, it can be concluded that the observed drift is most probably due to a general increase in vertical stratification as time progresses toward late spring.

It can be concluded from Figure 4 that between yearday 50 and 80, temperature is vertically homogeneous over the BASS height to less than $\pm 0.01^{\circ} \mathrm{C}$. Most of the temperature signal observed during these 30 days is associated with the $M_{2}$ tide advecting warm slope water upslope, or cool shelf water downslope depending on the direction of the tidal flow. Since shears are large in the vicinity of the seafloor, tidal 
excursion scales increase away from the bottom. The result is a weak vertical temperature gradient with temporal variations on the timescale of one tidal cycle. Photographs of the seafloor reveal that the near-bottom waters are clear, suggesting that vertical stratification due to sediment transport is not a factor. Assuming that salinity does not vary over the tripod height, it can be concluded from Figure 4 that the lowest part of the water column is to lowest order homogeneous during this time period. Based on this conclusion, the time period from yearday 50 to 80 will be analyzed in this study.

Time series of temperature, salinity, and $\sigma_{\theta}$ from Seacat data for yearday 50 to 80 are presented in Figure 6. It can be seen from the figure as well as from the temperature-salinity (T-S) characteristics in Figure 7 that temperature and salinity show similar behavior at all depths, with low-salinity waters playing a more important role closer to the surface. Time series of temperature, salinity, and potential density can be divided into three main segments: The first segment covers the period from yearday 50 to 60 , where large salinity values are combined with high, variable values for temperature. In the T-S characteristics (Figure 7), segment one manifests as a cloud in the upper right of the diagrams, with temperatures from $5.2-5.7^{\circ} \mathrm{C}$, and salinities around $33.2 \mathrm{psu}$. The second segment extends from yearday 60 to 69 . It is described by salinities of approximately $33 \mathrm{psu}$, and temperatures ranging from $5.2-5.5^{\circ} \mathrm{C}$ at heights 10.9 and $29.4 \mathrm{~m}$, and from $5.2-5.7^{\circ} \mathrm{C}$ at all other heights. Corresponding $\mathrm{T}-\mathrm{S}$ signatures can be found just underneath the cloud representing segment one and are still in the upper half of the diagrams. In both segments one and two, temperature displays a much larger variability than salinity, particularly in the lower part of the water column. This behavior leads to the conclusion that vertical stratification is small as long as temperature is homogeneous to lowest order. With mean temperature differences at heights 10.9 and $29.4 \mathrm{~m}$ being less than $\pm 0.01^{\circ} \mathrm{C}$ for both time segments, vertical stratification is negligible at these heights, indicating the presence of a bottom mixed layer of approximately $30 \mathrm{~m}$ thickness. The last time segment is from yearday 70 to 80 and displays salinities and temperatures smaller than those of segments one and two. Both salinity and temperature data show a decreasing trend toward the end of the time series, thus forming the tail in the lower left of the T-S characteristics, where a linear increase of salinity with temperature is found, especially at heights 10.9 and 
$29.4 \mathrm{~m}$. Therefore, salinity varies proportionally to temperature, again yielding the conclusion that potential density is vertically homogeneous as long as this is true for temperature. As before, temperature differences at 10.9 and $29.4 \mathrm{~m}$ above the bottom are smaller than $\pm 0.01^{\circ} \mathrm{C}$ in the time mean, so that vertical stratification is to lowest order negligible at these heights. At heights 49.9 to $74.5 \mathrm{~m}$, the signature of cold, fresh water with temperatures and salinities less than $4.7^{\circ} \mathrm{C}$ and $32.5 \mathrm{psu}$, respectively, is clearly visible in Figure 7. This suggests the intrusion of Scotian Shelf water (Bisagni, 1996), probably occurring towards the very end of the 30-day time series depicted in Figure 6. The significant decreases in temperatures and salinities at all depths between segments one and two as well as between segments two and three will not be investigated here, but might be associated with around-bank flow and intermittent intrusions of Scotian Shelf water.

Potential-density differences between adjacent Seacats for yearday 50 to 80 indicate that potential density is nearly homogeneous over the top $10 \mathrm{~m}$ of the water column, with the exception of some isolated events that may possibly be caused by the edges of Gulf Stream rings. If these events are excluded, mean buoyancy frequencies squared at 72.1 and $67.4 \mathrm{~m}$ above the bottom are $N^{2}<10^{-6} \mathrm{~s}^{-2}$ and $\approx 10^{-6} \mathrm{~s}^{-2}$, respectively. These values are representative for $83 \%$ of the 30 -day time period investigated in this study. Underneath the wind mixed layer, in the middle of the water column, potential-density differences in the vertical are more significant, and stratification of order $N^{2} \approx 1.7 \cdot 10^{-5} \mathrm{~s}^{-2}$ is observed. Approximately $20.1 \mathrm{~m}$ above the bottom, $N^{2}$ decreases to $10^{-6} \mathrm{~s}^{-2}$ indicating the presence of a bottom mixed layer and supporting the statements derived earlier from temperature and salinity data. Figure 8 shows profiles of mean temperature, salinity, and $\sigma_{\theta}$ over the time period from yearday 50 to 80 . The dashed line represents mean values obtained after isolated events of high stratification near the surface were excluded.

Richardson numbers computed from hourly averaged Seacat and VMCM data are smaller than 0.25 about $97 \%, 47 \%, 13 \%$, and $95 \%$ of the time period discussed above at the approximate heights $67.4,57.4,39.7$, and $20.1 \mathrm{~m}$, respectively. These results 
support the conclusion that a well-mixed bottom boundary layer exists, and that it extends to a height of at least $20 \mathrm{~m}$ above the seafloor.

In conclusion, the water column can be divided into three parts: A well-mixed, bottom boundary layer with a thickness of 20 to $30 \mathrm{~m}$, a weakly stratified interior with stratification $N^{2} \approx 1.7 \cdot 10^{-5} \mathrm{~s}^{-2}$, and a homogeneous surface-mixed layer of approximately $10-\mathrm{m}$ depth.

\section{II.3 Processing BASS Velocity Data}

In order to obtain good measurements of the veering and phase angles of the near-bed velocities, it is essential that the BASS sensors be mounted along the tripod axis with their transducers aligned in the vertical. However, this was not found to be perfectly true for the BASS1 deployment. Transducers of pods 1-3 in particular showed an offset of about $13.5^{\circ}$ counterclockwise from those of pods 4 and 5. Even with this error being corrected, inaccuracies in sensor alignment of $\pm 5^{\circ}$ or more must be expected, especially at pods 4 and 5 .

Before any further adjustments were made, differences in speed between adjacent acoustic current meters were examined as a function of flow direction for yearday 50 to 80 (Figure 9). Results of this investigation reveal that particularly at pods 2 and 3 , speeds decrease frequently with height when directions are between $-40^{\circ}$ and $125^{\circ}$ from east. Here, the minus sign denotes clockwise from east, and positive angles are counterclockwise from east. It can furthermore be seen that well-defined sectors of several degrees width exist where events of decreasing speeds between adjacent BASS sensors are frequent. Such sections are, for example, around $-165^{\circ},-90^{\circ}$, and $150^{\circ}$ in Figure 9c, and $-125^{\circ}$ in Figure 9d. Possible explanations for these events include tidal acceleration and flow obstruction due to instrumentation or bottom topography.

To investigate the effects of tidal acceleration on the bottom boundary layer, current profiles taken at times of increasing and decreasing speeds $U$ of the dominant $\mathrm{M}_{2}$ tidal constituent are analyzed. Tidal analysis shows that near the seafloor, the inclination of the $\mathrm{M}_{2}$ ellipse is about $-65^{\circ}$ from east, and the ratio of minor and 
major axes is approximately 0.62 . With $\frac{\partial U}{\partial t}$ being zero if the flow is along the major or minor axis, and with the rotation of the tidal ellipse being clockwise, $\frac{\partial U}{\partial t}$ is greatest around $-20^{\circ}$ and $160^{\circ}$ from east, and smallest around $-110^{\circ}$ and $70^{\circ}$ from east. Figure 10 shows current profiles taken at these angles, where a semi-logarithmic scale is chosen to depict speed as a function of height. Velocity data shown in the figure are averaged data, with the average being taken over events where the flow is within $\pm 5^{\circ}$ from the directions given above. The dashed line represents results from a leastsquares fit to the logarithmic velocity profile, given by

$$
U=\frac{u_{*}}{\kappa} \ln \left(\frac{z}{z_{0}}\right),
$$

where

$$
u_{*}=\sqrt{\frac{\tau_{b}}{\rho_{0}}} .
$$

In (1) and (2), $u_{*}$ is friction velocity, $\kappa$ is von Karman's constant taken as 0.4, $z$ is height above the bottom, $z_{0}$ is bottom roughness, and $\tau_{b}$ is the magnitude of the kinematic bottom stress. The least-squares fit is made to speeds measured at pods 1-4 after applying the averaging described above. Velocities measured at pod 5 are not included in the fit, since it is questionable whether the logarithmic layer extends to that height. According to Soulsby et al. (1981), in a rectilinear tidal flow, speeds near the sea floor are smaller than the logarithmic value given above during times of acceleration, and they are larger than this value during times of deceleration. The deviation from the logarithmic profile increases with height. This behavior is explained by the fact that the relative importance of friction is greater near the bed than away from the bed, forcing velocities to be more in phase with the pressure gradient as the boundary is approached. Although these results were derived for rectilinear flows, similar behavior can be expected for rotational flows as long as the veering of the major axis is small over the boundary layer height, and the tidal ellipse is not close to circular. Both statements are true for the data discussed in this study, but none of the effects of time-varying flow on the logarithmic velocity profile are evident in Figure 10. Thus, it can be concluded that the relative importance of acceleration and deceleration for the logarithmic layer is negligible at heights less than $4.5 \mathrm{~m}$. It is therefore unlikely that the 
events of vertically decreasing speeds observed in Figure 9 are related to accelerating flow, especially since many of these events do not occur at angles where acceleration is large. With this information, it is now possible to apply further adjustment to the velocity data measured by the BASS.

Even with the best pre-deployment dock calibration to determine sensor velocity zeros, profiles of speeds measured by BASS show consistent offsets relative to a best fit logarithmic profile (Gross, personal communication). In the absence of other sources of error, and if deviations from the logarithmic profile due to the tidal oscillation as well as stratification are negligible, these residuals represent the final error of each velocity sensor and occur as a function of direction. In order to correct for the offset residuals, a method developed by Gross is applied to the BASS1 velocity data taken from yearday 50 to 80 . In this procedure, the mean horizontal residual from the logarithmic fit is found as a function of direction for all pods, and a sine wave is fitted to the average residuals. Since it can be concluded from Figure 10 that deviations from the best fit logarithmic profile are small at pod 5 , it is reasonable that pod 5 be included in the fit for the purpose of finding the offset residuals. The magnitude of the sine wave and its direction describe the residual vector, which is then removed from the original data. Residuals for pods $1-5$ are of order $1 \mathrm{~cm} / \mathrm{s}$ or less, with measured flow speeds being between 5 and $40 \mathrm{~cm} / \mathrm{s}$. The largest residuals are computed for pod 2. This is in accordance with the fact that vertical velocities measured at pod 2 are of order $1 \mathrm{~cm} / \mathrm{s}$, a value that is high compared to other pods and indicates insufficient or faulty dock calibration. In Figure 11, current profiles similar to those in Figure 10 are presented, but with the data being adjusted according to the method described above. It can be seen from the figure that the adjustment does not affect the validity of statements about unsteady tidal flow made earlier, and that the logarithmic fit has been improved. The latter is especially a result of the correction made at pod 2. The impact of this correction is particularly evident from investigation of the subtidal flow, where long time periods of decreasing speeds between pods 1 and 2 are observed in the original data, but disappear after the adjustment is made. Overall, the number of events where speeds decrease with height is reduced by $40 \%$ if the offset residuals are removed, mostly due to the correction at pod 2. However, other such events, much as shown in Figure 9, are 
still present and remain almost insensitive to the adjustment. Attempts were made to relate these directions to flow obstructions caused by tripod legs and instrumentation, but no conclusions can be drawn at this point. In the following analysis, only the adjusted data were used. However, tests have been carried out to ensure that results are not sensitive to the correction applied to the BASS velocity data described above.

\section{II.4 Estimating Bottom Stress}

\section{II.4.1 Friction Velocity}

According to (1), the friction velocity $u_{*}$ is estimated by taking the instantaneous best fit logarithmic profile to the burst-averaged speeds measured at pods $1-4$. The fit is done for events where speeds at all four velocity sensors increase away from the bottom, since the logarithmic law of the wall is not valid if speeds decrease with height. This limits the data to be used for estimating bottom stress to approximately $80 \%$ of the complete data set for yearday 50 to 80 . As time gaps between logarithmic fits are in general no longer than $0.5-1$ hour, it is reasonable to find missing values for $u_{*}$ by interpolation. Although this is not an issue for the statistics discussed in this paragraph, interpolation will be carried out at a later point to produce a complete time series of bottom stress for tidal analysis. However, tests show that even if all data are included in the logarithmic fit, none of the statistics discussed in this paragraph, or the results presented in subsequent chapters will be subject to noticeable changes.

The time-averaged linear correlation coefficient of all logarithmic fits is $0.97 \pm 0.03$, where the first value represents the time mean, and the second value is the standard deviation from the mean. Corresponding results for $u_{*}$ are $1.2 \pm 0.4 \mathrm{~cm} / \mathrm{s}$. The standard deviation of the friction velocity is mainly caused by the $M_{2}$ tide, which leads to large $u_{*}$ if speeds are high, and small $u_{*}$ if speeds are low. Figure 12 presents $u_{*}$ as a function of time. Scatterplots of $u_{*}$ and $\frac{\Delta u_{*}^{95}}{u_{*}}$ versus speed at pod 3 are shown in Figure 13, where $\Delta u_{*}^{95}$ is the $95 \%$ confidence interval of $u_{*}$. It can be seen from Figure 13a that $u_{*}$ shows a linear increase with speed, very much 
as suggested by the quadratic drag law

$$
\frac{1}{\rho_{0}} \tau_{b}=c_{D} U^{2}
$$

where

$$
c_{D}=\left[\frac{\kappa}{\ln \left(\frac{z}{z_{0}}\right)}\right]^{2} .
$$

in agreement with the logarithmic law of the wall. In (3) and (4), $c_{D}$ is the drag coefficient. Least-squares fits of $u_{*}^{2}$ versus $U^{2}$ at $0.22,0.58,1.18$ and $2.53 \mathrm{~m}$ height above the bottom give $c_{D}=(4.34 \pm 0.07) \cdot 10^{-3}, c_{D}=(3.57 \pm 0.06) \cdot 10^{-3}, c_{D}=$ $(2.92 \pm 0.05) \cdot 10^{-3}$, and $c_{D}=(2.39 \pm 0.04) \cdot 10^{-3}$, respectively, where the second value denotes the error of the fit. Standard deviations from the fits are $4.91 \cdot 10^{-3}, 2.27 \cdot 10^{-3}$, $1.83 \cdot 10^{-3}$, and $1.17 \cdot 10^{-3}$ at the same heights, respectively.

\section{II.4.2 Bottom Roughness}

The second parameter computed from the best fit logarithmic profiles is the bottom roughness, $z_{0}$. Values for $z_{0}$ obtained from the fit range between $7 \cdot 10^{-7} \mathrm{~cm}$ to $9.2 \mathrm{~cm}$. Scatterplots of $\log _{10}\left(z_{0}\right), \frac{\Delta z_{0}^{95+}}{z_{0}}$, and $\frac{\Delta z_{0}^{95-}}{z_{0}}$ versus $u_{*}$ are shown in Figure 14 . Here, $\Delta z_{0}^{95+}$ and $\Delta z_{0}^{95-}$ are the upper and lower $95 \%$ confidence intervals for $z_{0}$, respectively. It is obvious from Figure 14a that unrealistically low estimates of $z_{0}$ may result if values for $u_{*}$ are small. A physical lower limit for $z_{0}$ is given by the height of the viscous sublayer, in general taken as

$$
\delta_{\nu} \approx \frac{5 \cdot \nu}{u_{*}}
$$

where $\nu$ is molecular viscosity. With $\nu$ being approximately $10^{-2} \frac{\mathrm{cm}^{2}}{\mathrm{~s}}$, and with $u_{*} \approx \bar{u}_{*} \approx 1.2 \mathrm{~cm} / \mathrm{s}$ as given above, the viscous sublayer thickness is of order $0.4 \mathrm{~mm}$. Pictures of the seafloor reveal the presence of sand ripples during the entire time period from yearday 50 to 80 (Figure 15). Ripples are always in the same direction, with their crest aligned northeastward, and do seem to be of comparable height at all times. 
According to Grant et al. (1982), the bottom roughness over a hydrodynamically fully rough boundary is given by

$$
z_{0}=\frac{k_{s}}{30},
$$

where $k_{s}$ is the equivalent sand roughness approximated by

$$
k_{s}=30 \cdot h \cdot \frac{h}{\lambda} .
$$

In (7), $h$ is the height of the sand ripples, and $\lambda$ is their separation distance. Based on Figure 15, a reasonable estimate for $\lambda$ is between 15 and $20 \mathrm{~cm}$. The height of the crests is more difficult to determine, but needs to be of order 1-2 cm for ripples to be clearly visible on photographs. According to (6) and (7), these values yield bottom roughness lengths between 0.7 and $2.7 \mathrm{~mm}$, indicating once more that estimates much less than this order of magnitude are not realistic.

Large estimates of $z_{0}$ are found for current directions between -35 and $-75^{\circ}$ from east and may be associated with flow distortion due to the battery powering the camera and light. The battery case is attached to the first BASS platform, between the camera and light, and represents an obstruction of considerable size to northwestward flow (Figure 3). As a result, increased turbulence may occur, particularly affecting the measurements taken at pods $1-3$. Although this does not seem to show significant influence on estimates of $u_{*}$, estimates of $z_{0}$ are more sensitive, and uncharacteristically large for southeasterly flow. Histograms of $\log _{10}\left(z_{0}\right)$ are presented in Figure 16, where current directions believed to be affected by the battery are included in 15a, and excluded in 15b. Comparison of Figure 16a and b reveals that more than $60 \%$ of the estimates for $z_{0}$ of order $1.2 \mathrm{~mm}$ and larger occur when the flow is from directions excluded in $b$, while the number of estimates smaller than this value is changed by less than $5 \%$. According to Figure $16 \mathrm{~b}$, a reasonable value for the physical bottom roughness is around $0.6-0.8 \mathrm{~mm}$ if estimates smaller than $0.4 \mathrm{~mm}$ are ignored based on the arguments given above. However, it is difficult to infer an accurate estimate of bottom roughness from Figure $16 \mathrm{~b}$ due to the wide range of $z_{0}$ obtained from the best-fit logarithmic profiles. A representative value for $z_{0}$ can be derived from (4), 
with $c_{D}$ being determined by fitting $U^{2}$ to $u_{*}^{2}$ in a least-squares sense. If current speeds measured at pods 1-4 are used to compute the fit, results for $z_{0}$ are $0.5,0.7$, 0.7 , and $0.7 \mathrm{~mm}$ corresponding to the drag coefficients at heights $0.22,0.58,1.18$, and $2.53 \mathrm{~m}$ above the bottom, respectively. The average bottom roughness according to these values is $z_{0} \approx 0.7 \mathrm{~mm}$, and will be implemented as a characteristic value in the numerical modeling study discussed later.

It was first believed that the presence of sand ripples, possibly migrating with the flow, may account for the observed increase of $z_{0}$ if the flow is on-bank. However, as described earlier, ripples are always aligned to the northeast, and roughly perpendicular to the major axis of the tidal ellipse. They do not rotate with the tidal current, so that the flow is at right angles to the crests twice during one tidal cycle. Therefore, any variations in $z_{0}$ introduced by the relative direction of current velocities to sand ripples should occur twice within one tidal cycle. This is in contradiction with the actual observations, which show that $z_{0}$ increases when the flow is on-bank, but not when it is off-bank. Thus, flow distortion due to the battery case is more likely an explanation for the observed periodical variation of estimated bottom roughness.

According to Grant et al. $(1979,1986)$, estimates of $u_{*}$ and $z_{0}$ might be affected by surface waves at times where bottom wave velocities are high. If near-bottom orbital velocities obtained from linear wave theory are large compared to current speeds, a wave-induced boundary layer of several centimeters height can build up just above the seafloor. This wave boundary layer represents a region of wave-induced turbulence and increases the apparent bottom roughness experienced by the current above it. In order to examine the role of the wave boundary layer as a possible cause for high estimates of $z_{0}$, bottom roughness length is plotted versus the ratio of bottom orbital velocity, $u_{b}$, to the pod 1 current speed, $U_{1}$, (Figure 17). Bottom orbital velocities were derived from hourly wave amplitude and period data taken at the NDBC environmental buoy 44011 located at $41^{\circ} 6^{\prime} \mathrm{N}, 66^{\circ} 36^{\prime} \mathrm{W}$. The water depth at the buoy location is approximately $86 \mathrm{~m}$. Wave amplitudes at the shallower ST1-site are computed assuming energy conservation with no dissipation, and are almost identical to those measured by the buoy. It is apparent from Figure 17 that only a few events of 
significantly large near-bottom orbital velocities were observed. In fact, $\frac{u_{b}}{U_{1}}$ is greater than 0.1 approximately $10 \%$ of the time, and greater than 0.2 approximately $3 \%$ of the time. Events with $\frac{u_{b}}{U_{1}}>0.1$ do not coincide with high estimates of $z_{0}$ to a greater extent than those where $\frac{u_{b}}{U_{1}}$ is small. Thus, no correlation between surface waves and apparent bottom roughness was found.

\section{II.4.3 Bottom Stress Vector}

Based on the results from best-fit logarithmic profiles to current speeds at pods 14 , the magnitude of the bottom stress vector may be computed from values $u_{*}^{2}$ according to (2). Bottom stress estimates can also be obtained from the quadratic drag law (3), if characteristic values for $c_{D}$ are available. This method requires that assumptions be made concerning the specification of a constant drag coefficient, and the height at which the drag law is to be applied.

In the present case, the periodical variation of estimated bottom roughness associated with the battery case indicates that bottom stress estimates from best-fit logarithmic profiles may be subject to similar, physically unrealistic fluctuations. Investigation shows that application of either (2) or (3) to compute the bottom stress magnitudes leads to results that differ by less than $5 \%$ at the frequency of the $\mathrm{M}_{2}$ tide. On the other hand, differences in both magnitude and direction are substantial when computing the subtidal bottom stress, since the periodical variation occuring once during a $\mathrm{M}_{2}$ cycle introduces a subtidal component which is physically unrealistic. In order to avoid the implemention of additional assumptions associated with the quadratic drag law to the greatest extent possible, bottom stress magnitudes $\frac{1}{\rho_{0}} \tau_{b}=u_{*}^{2}$ were used in the analysis of the total and $\mathrm{M}_{2}$ currents, while values $\frac{1}{\rho_{0}} \tau_{b}=c_{D} U^{2}$ were computed for the analysis of the subtidal flow. The quadratic drag law was applied to pods 1-3, and the results from the three pods were averaged to obtain a time series of bottom stress estimeates. Drag coefficients were obtained from least-squares fits of $U^{2}$ to $u_{*}^{2}$ as described above. 
The direction of the bottom stress vector was taken as the vertical average over the flow directions measured at pods 1-3. Although possible uncertainties in sensor alignment render quantitative statements about the rotation of the velocity vector with height difficult, data indicates that in the time mean, flow orientations between pods 1 and 3 differ by less than $3^{\circ}$. Thus, it is reasonable to conclude that the bottom stress is parallel to the instantaneous flow at these heights within measurement error. 


\section{Vertical Structure of the $\mathrm{M}_{2}$ Tidal Flow}

\section{III.1 Introduction}

In this chapter, the current and stress ellipses of the dominant tidal constituent, the $\mathrm{M}_{2}$, will be analyzed and discussed. Other tidal constituents are small compared to the $\mathrm{M}_{2}$ and will not be mentioned in great detail. The chapter focuses on scaling arguments for boundary layer heights and transports associated with the $M_{2}$ tidal flow, bottom stresses at the $M_{2}$ frequency, and eddy viscosity profiles. Two sections are included: one presenting results from data analysis, and one comparing these results to one-dimensional numerical model predictions.

\section{III.2 Data Analysis}

\section{III.2.1 Tidal Current Analysis}

A tidal current analysis was carried out on the hourly averaged data obtained from BASS and VMCM measurements for the time period from yearday 50 to 80 using the Foreman (1978) code. This code computes the complex rotary components of each resolvable tidal constituent, the magnitudes of the major and minor axes of the corresponding tidal ellipses, and the phase and inclination angles of the major axes. Results are obtained by fitting the sum of all constituents that can be resolved for the given time period to the measured data in a least-squares sense, according to

$$
u+i \cdot v=\sum_{j=1}^{M}\left(\mathbf{R}^{+} e^{i \sigma_{j} t}+\mathbf{R}^{-} e^{-i \sigma_{j} t}\right) .
$$

In (8), $u$ and $v$ are the measured eastward and northward velocities, respectively, $M$ is the number of resolvable tidal constituents, $\sigma_{j}$ are the frequencies of the constituents, $t$ is time, and $\mathbf{R}^{+}$and $\mathbf{R}^{-}$are the complex anti-clockwise and clockwise rotating components, respectively. The rotary components may also be written in the 
form

$$
\mathbf{R}^{ \pm}=R^{ \pm} e^{i \phi^{ \pm}}
$$

where $R^{ \pm}$is magnitude, and $\phi^{ \pm}$is phase angle of the respective component. Major and minor axes of the tidal ellipses can be computed from

$$
U_{M a j}=R^{+}+R^{-}
$$

and

$$
U_{M i n}=R^{+}-R^{-}
$$

The current ellipse rotates anti-clockwise for $U_{M i n}>0$ and clockwise for $U_{M i n}<0$. Inclination and phase of the major axis are given by

$$
\theta=0.5 \cdot\left(\phi^{+}+\phi^{-}\right)
$$

and

$$
\phi=0.5 \cdot\left(\phi^{+}-\phi^{-}\right)
$$

respectively. Since both $\phi^{+}$and $\phi^{-}$can be altered by $360^{\circ}$ without changing the representation of the original current, an ambiguity of $180^{\circ}$ for inclination and phase is introduced in (11a) and (11b). In the following, this ambiguity is avoided by choosing the off-bank major axis as the reference axis. The time that has elapsed since the current vector passed this reference axis is given by $t=\frac{\phi}{\sigma}$, where $\sigma$ is the frequency of the respective constituent. A more detailed description of rotary components and tidal ellipse parameters is given in Soulsby $(1983,1990)$.

The tidal analysis code was used in its original form, with some minor alterations: First, nodal corrections to the ellipse parameters are negligible and were omitted. Second, phases are given with respect to the beginning of the time series, here midnight on yearday 49. Third, the off-bank major axis is chosen as the reference axis. And fourth, uncertainties of the ellipse parameters are computed from the spectrum of the current residuals summed over a given frequency band, and divided by the band width. For the semidiurnal band, the band width was taken as $\pm 0.11 \cdot 10^{-4} \frac{1}{\mathrm{~s}}$ centered around the 
$\mathrm{M}_{2}$ frequency, $\sigma_{M_{2}}=1.41 \cdot 10^{-4} \frac{1}{\mathrm{~s}}$. Uncertainties computed in this manner account for errors due to the least-squares fit of the tidal constituents to random errors in the measured flow, but do not take into consideration systematic measurement errors. It is unknown to what extent measurement uncertainties affect estimated current magnitudes in the different frequency bands. However, tests show that the results presented here are insensitive to a random error of $\pm 2 \mathrm{~cm} / \mathrm{s}$ in the hourly data, a value representative of the uncertainty of the VMCM (Lentz, personal communication). Because compass errors of BASS and VMCMs may be as large as $\pm 15^{\circ}$ and $\pm 5^{\circ}$, respectively, it must be assumed that uncertainties of ellipse phases and inclinations cannot be smaller than these values. The compass error of BASS applies similarly to all acoustic current meters.

Nineteen distinct frequencies can be resolved for the time period from yearday 50 to 80 , those being the frequencies of the $Z_{0}, M S f$ (spring-neaps cycle), $Q_{1}, O_{1}$, $K_{1}, J_{1}, N_{2}, M_{2}, S_{2}, M O_{3}, M_{3}, M K_{3}, M N_{4}, M_{4}, M S_{4}, S_{4}, M K_{5}, M_{6}$, and $2 M S_{6}$ constituents. From all constituents, the $\mathrm{M}_{2}$ is, by far, the most energetic and contains about $88 \%$ of the total kinetic energy. Since all other constituents are small, it must be expected that they are not resolved with great accuracy. This is true for the $M S f$ in particular, since it evolves on a time scale long enough for fluctuations caused by surface winds, or advection associated with Gulf Stream rings to be captured in the same frequency band. The combined effects of all constituents with frequencies less than diurnal will therefore be considered subtidal, and account for approximately $6 \%$ of the total kinetic energy. The next largest contributions are from the $S_{2}, N_{2}$, and $K_{1}$ constituents, containing $\approx 3 \%, 2.5 \%$, and $0.5 \%$ of the kinetic energy, respectively.

\section{III.2.2 The $\mathrm{M}_{2}$ Current Ellipse}

The vertical structure of the $M_{2}$ tidal velocities at different times during the tidal cycle is shown on a semi-logarithmic scale in Figure 18. Profiles are from averaged data, where the average was taken of all current measurements with flow directions less than $\pm 5^{\circ}$ from angles of maximum acceleration and deceleration of the near-surface tidal speeds. Dotted lines in Figure 18 represent the total current speeds averaged in the 
same manner, and dashed lines are results from a least-squares logarithmic fit to velocity data taken at pods $1-4$. Velocity measurements at $30.5 \mathrm{~m}$ above the bottom exceed those taken at greater heights by more than $2 \mathrm{~cm} / \mathrm{s}$ during times of flow acceleration (Figure 18a,c). It is believed that this behavior is caused by an instrumentation offset, resulting in current measurements that are consistently too large. The convex curvature of the $\mathrm{M}_{2}$ velocity profiles during times of accelerating speeds (Figure 18a,c), and the concave curvature during times of decelerating speeds (Figure 18b,d) suggest that surface tidal currents lag the near-bottom velocities. As described in Chapter II, this behavior is explained by the fact that the relative importance of friction increases with decreasing distance from the seafloor, causing the flow to be more in phase with the pressure gradient and less in phase with inertia as it approaches the bottom. Since the flow field is dominated by the $\mathrm{M}_{2}$ tide, similar conclusions can be drawn from velocity profiles of the total flow. Low-pass-filtered velocities obtained from the PL64 (Beardsley et al., 1983) are predominantly to the southwest (Figure 19), where the low pass applies to frequencies smaller than diurnal. Thus, the total flow is in general greater than the tidal flow at angles $-103^{\circ}$ and $167^{\circ}$ (Figure $18 \mathrm{~b}, \mathrm{c}$ ), and smaller than the tidal flow at angles $-14^{\circ}$ and $77^{\circ}$ (Figure 18a,d). Subtidal flow speeds typically increase towards the surface, causing the effects of tidal acceleration to be less apparent if their direction is at acute angles with the tidal current (Figure 18c).

Magnitude and phases of the $\mathrm{M}_{2}$ rotary components, major and minor axes of the tidal ellipse, and inclination and phase of the major axis are shown as a function of height in Figure 20. It is evident from Figure 20a that $R^{-}$by far exceeds $R^{+}$at all depths, causing the current ellipse to rotate clockwise. The eccentricity of the depthaveraged tidal ellipse is $e=\frac{U_{M i n}}{U_{M a j}}=0.64$. Current shears are greatest near the bottom, where friction due to bottom stresses is large. This is apparent from the magnitudes of rotary components and current amplitudes, as well as from the phase angles $\phi^{+}$and $\phi^{-}$. Since $\mathbf{R}^{+}$rotates anticlockwise and $\mathbf{R}^{-}$clockwise, associated boundary layer transports are to the left of $\mathbf{R}^{+}$and to the right of $\mathbf{R}^{-}$, resulting in anticlockwise and clockwise veering of $\phi^{+}$and $\phi^{-}$away from the bottom, respectively. This behavior can clearly be observed in Figures $20 \mathrm{~b}$ and c, with the veering being strongest in the vicinity of the sea floor. The vertical structure of both $\phi^{+}$and $\phi^{-}$combines 
to give the vertical structure of inclination and phase angles of the current ellipse, shown in Figures 20e and f. According to Figure 20d, the major axis of the tidal ellipse rotates clockwise as the bottom is approached. Overall, changes in inclination between the surface and the bottom are small $\left(<10^{\circ}\right)$. Due to the large compass error and possibility of inaccurate sensor alignment of BASS, the orientation of veering near the seafloor cannot be concluded with certainty. However, Figure 20e suggests that inclination does not change significantly within the lowest five meters. Phases of the current ellipse near the bottom lead those at the surface by approximately $20^{\circ}$, as shown in Figure 20f. This is consistent with the results from $M_{2}$ velocity profiles discussed earlier.

In the upper part of the water column at heights larger than 40 to $50 \mathrm{~m}$, current amplitudes, phase angles, and inclination approach constant values and no longer display significant changes in the vertical. At these heights friction is negligible, since bottom stresses are balanced in the lower part of the water column. Surface stresses have no contribution in the semidiurnal and diurnal frequency bands, and therefore do not affect the $M_{2}$ tidal currents. This is obvious from the kinetic energy density spectrum of the wind shown in Figure 21. In the absence of friction and under the assumption that nonlinear advective terms are small, the momentum equations for homogeneous flow, written in rotary components, are given by

$$
\begin{aligned}
i(f+\sigma) \mathbf{R}_{\infty}^{+} & =-\mathbf{P}^{+} \\
i(f-\sigma) \mathbf{R}_{\infty}^{-} & =-\mathbf{P}^{-} .
\end{aligned}
$$

In (12), $\quad \mathbf{R}_{\infty}^{ \pm}$denote the rotary components of the free-stream velocities, $\sigma=$ $1.41 \cdot 10^{-4} \frac{1}{\mathrm{~s}}$ is the frequency of the $\mathrm{M}_{2}$ tide, $f=9.5 \cdot 10^{-4} \frac{1}{\mathrm{~s}}$ is the Coriolis parameter, and $\mathbf{P}^{ \pm}$are the rotary components of the pressure gradient divided by density. The assumption that density be considered vertically homogeneous was shown to be reasonable in Chapter II. Horizontal density gradients may be present, but evolve on time scales comparable to seasonal variations. They do not contribute to pressure gradients with semidiurnal and diurnal frequencies, so that in (12), $\mathbf{P}^{ \pm}$represents the barotropic pressure forcing of the $M_{2}$ tide. Rossby numbers based on $M_{2}$ current amplitudes are small, justifying that advective terms be neglected. With depth-averaged 
current amplitudes of $40 \mathrm{~cm} / \mathrm{s}$ and a characteristic horizontal length scale $L \approx 40 \mathrm{~km}$, Rossby numbers for the depth averaged flow are of order $\epsilon=\frac{U}{f L} \approx 0.1$. The horizontal length scale is set by bottom topography, and is taken as the across-bank distance over which the bottom slope remains approximately constant (Figure 2). Taking $\mathbf{R}_{\infty}^{ \pm}$as the average value of all rotary components computed at heights greater than $45 \mathrm{~m}$, the major and minor axes of the pressure ellipse, $P_{M a j}$ and $P_{M i n}$, are $3.40 \cdot 10^{-1} \mathrm{~cm}^{2} \mathrm{~s}^{-2}$ and $0.13 \cdot 10^{-1} \mathrm{~cm}^{2} \mathrm{~s}^{-2}$, respectively. The inclination of $P_{M a j}$ is approximately $-59^{\circ}$ from east, corresponding to the inclination of the current ellipse near the surface (Figure 20e). With the eccentricity $e=\frac{P_{M i n}}{P_{M a j}}$ of the pressure ellipse being approximately 0.04 , the $\mathrm{M}_{2}$ pressure forcing is almost rectilinear and oriented across isobath.

Results concerning current amplitudes, phase, and inclination of the $\mathrm{M}_{2}$ tidal ellipse are consistent with those obtained for other semidiurnal and diurnal constituents (Figures 22-24). In all cases, the tidal ellipse rotates clockwise, with $R^{-}$by far exceeding $R^{+}$. Although the orientation of the $\mathrm{N}_{2}$ current ellipse does not display significant changes in the vertical (Figure 22e), the inclination of the $\mathrm{S}_{2}$ ellipse indicates anticlockwise veering as the surface is approached (Figure 23e). If error bars are neglected, the rotation between seafloor and surface amounts to approximately $15^{\circ}$, a value comparable to that observed for the $\mathrm{M}_{2}$ tide. Again ignoring error bars, phase angles of both the $N_{2}$ and the $S_{2}$ constituents lead those near the bottom by roughly $20^{\circ}$, similar to the behavior of the $\mathrm{M}_{2}$ currents. Inclination and phase of the $\mathrm{K}_{1}$ constituent display large uncertainties and shall not be discussed here.

\section{III.2.3 Boundary Layer Height Scaling}

It is evident from Figures 20a-c that the vertical structure of the bottom boundary layer is described by two distinct boundary layer heights, $\delta^{+}$and $\delta^{-}$, set by the anticlockwise and clockwise rotating components, respectively. Magnitudes as well as phase angles of $\mathbf{R}^{+}$and $\mathbf{R}^{-}$indicate that the clockwise component occupies a much thicker boundary layer than the anticlockwise component. However, it is difficult to give an unambiguous definition of the corresponding boundary layer heights, since they 
depend on the physical parameter examined as well as on specifications concerning the nearness to free-stream values. For example, magnitudes $R^{+}$and $R^{-}$have reached approximately $95 \%$ of their free-stream values at heights $\delta^{+} \approx 8 \mathrm{~m}$ and $\delta^{-} \approx 40 \mathrm{~m}$, respectively (Figure 20a). In determining these values, it was taken into account that velocity measurements at $30.5 \mathrm{~m}$ above the bottom are believed to over-estimate the actual current speeds by $2-3 \mathrm{~cm} / \mathrm{s}$. From Figures $20 \mathrm{~b}$ and c, it can be seen that the veering of the corresponding phase angles $\phi^{+}$and $\phi^{-}$occurs almost entirely at heights smaller than 50 and $10 \mathrm{~m}$, respectively. The significant difference between the two boundary layer thicknesses $\delta^{+}$and $\delta^{-}$is explained by the opposite-direction rotation of $\mathbf{R}^{+}$and $\mathbf{R}^{-}$. While the cyclonic rotation of $\mathbf{R}^{+}$assists the Coriolis acceleration in balancing the bottom stresses, the anticyclonic rotation of $\mathbf{R}^{-}$opposes it. Thus, effects of friction extend to greater heights for $\mathbf{R}^{-}$than for $\mathbf{R}^{+}$, a fact that manifests itself in $\delta^{-}>\delta^{+}$.

Scaling arguments for the boundary layer thicknesses of the rotary components can be derived from the momentum equations

$$
\begin{aligned}
& i(f+\sigma) \mathbf{R}^{+}=-\mathbf{P}^{+}+\frac{\partial \tau^{+}}{\partial z}, \\
& i(f-\sigma) \mathbf{R}^{-}=-\mathbf{P}^{-}+\frac{\partial \tau^{-}}{\partial z} .
\end{aligned}
$$

In (13), $\quad \boldsymbol{\tau}^{ \pm}$are the rotary components of the stress vector as a function of $z$ divided by density. Substituting $\tau^{ \pm}=K \cdot \frac{\partial \mathbf{R}^{ \pm}}{\partial z}$, where $K$ is an eddy viscosity, yields

$$
\begin{aligned}
& i(f+\sigma) \mathbf{R}^{+}=-\mathbf{P}^{+}+\frac{\partial}{\partial z}\left(K \frac{\partial \mathbf{R}^{+}}{\partial z}\right), \\
& i(f-\sigma) \mathbf{R}^{-}=-\mathbf{P}^{-}+\frac{\partial}{\partial z}\left(K \frac{\partial \mathbf{R}^{-}}{\partial z}\right) .
\end{aligned}
$$

Solutions to (14) depend on assumptions concerning the magnitude, vertical structure, and temporal variation of $K$. For the simplest case, $K=$ constant, and if the no-slip condition is applied at the sea floor, $\mathbf{R}^{ \pm}$are given by (Sverdrup, 1927; Fjeldstad, 
1929; Mofjeld, 1980; Kundu et al., 1981; Fang et al., 1983)

$$
\begin{aligned}
& \mathbf{R}^{+}=\mathbf{R}_{\infty}^{+}\left(1-e^{-\frac{(1+i)}{\sqrt{2}}\left(\frac{\sigma+f}{K}\right)^{1 / 2} z}\right), \\
& \mathbf{R}^{-}= \begin{cases}\mathbf{R}_{\infty}^{-}\left(1-e^{-\frac{(1+i)}{\sqrt{2}}\left(\frac{\sigma-f}{K}\right)^{1 / 2} z}\right) & \text { for } \sigma>f, \\
\mathbf{R}_{\infty}^{-}\left(1-e^{-\frac{(1-i)}{\sqrt{2}}\left(\frac{f-\sigma}{K}\right)^{1 / 2} z}\right) & \text { for } \sigma<f .\end{cases}
\end{aligned}
$$

Equation (15) implies that boundary layer heights scale as

$$
\delta^{ \pm}=c \cdot\left|\frac{K}{\sigma_{ \pm} f}\right|^{1 / 2} .
$$

where $c$ is a constant which depends on the specifications concerning the nearness to free-stream conditions. In principle, (16) represents nothing else than the classic Ekman scaling height applied to rotary components, where the Coriolis parameter $f$ is replaced by $\sigma+f$ and $|\sigma-f|$ for the anticlockwise and clockwise component, respectively.

The fact that turbulent motions are restricted by the bottom boundary can be modeled by allowing the eddy viscosity to increase linearly away from the seafloor. According to Tennekes (1973),

$$
K=\frac{u_{*}^{2}}{\frac{\partial u}{\partial z}}=\kappa u_{*} z
$$

is consistent with the logarithmic law of the wall, and is valid near the boundary. Under the simplifying assumptions that (17) holds throughout the entire water column, and that $u_{*}$ can be represented by a constant value such as the maximum friction velocity during one tidal cycle, $\hat{u}_{*}$, solutions to (14) are (Prandle, 1982; Soulsby, 1983; Ostendorf, 1984)

$$
\mathbf{R}^{ \pm}=\mathbf{R}_{\infty}^{ \pm}\left[\left(1-\frac{\operatorname{ker} \xi \operatorname{ker} \xi_{0}+\operatorname{kei} \xi \operatorname{kei} \xi_{0}}{\operatorname{ker}^{2} \xi_{0}+\operatorname{kei}^{2} \xi_{0}}\right) \pm i\left(\frac{\operatorname{ker} \xi \operatorname{kei} \xi_{0}-\operatorname{kei} \xi \operatorname{ker} \xi_{0}}{\operatorname{ker}^{2} \xi_{0}+\operatorname{kei}^{2} \xi_{0}}\right)\right]
$$


where $\xi=2 \cdot\left|\frac{(f \pm \sigma) z}{\kappa \hat{u}_{*}}\right|^{1 / 2}, \xi_{0}=2 \cdot\left|\frac{(f \pm \sigma) z_{0}}{\kappa \hat{u}_{*}}\right|^{1 / 2}$, and ker and kei are Kelvin functions of zeroeth order. In deriving (18), the no-slip condition has been applied at the bottom. Equation (18) suggests meaningful scale heights for the boundary layer thicknesses to be

$$
\delta^{ \pm}=c \cdot \frac{\kappa \bar{u}_{*}}{|\sigma \pm f|}
$$

where again the constant $c$ is ambiguous, and a characteristic value for the friction velocity is given by its mean value during one tidal cycle, $\bar{u}_{*}$. Equation (19) is in agreement with results for planetary boundary layers and oscillatory flows, if the denominator is replaced by the corresponding frequencies, the Coriolis parameter, $f$, and the frequency of the rectilinear flow, $\omega$, respectively. Limited observational evidence for neutral planetary boundary layers gives the boundary layer height $\delta \approx \frac{\kappa \cdot u_{*}}{f}$ (Grant et al., 1986). Laboratory results for non-rotating, steady flows (Clauser, 1956) indicate that the logarithmic layer occupies about $10 \%$ of the total boundary layer thickness. Similar results were obtained from numerical and experimental investigation of purely oscillatory flows (Grant, 1977; Grant et al., 1986; Johnson et al., 1976). The neutral boundary layer height of such flows was found to scale as $\delta \approx \frac{\kappa \cdot \hat{u}_{*}}{\omega}$, where $\omega$ is the frequency of the oscillation. The logarithmic-layer thickness is approximately $0.1 \delta$, in agreement with Clauser's (1956) results. Grant et al. (1986) point out that the eddy viscosity cannot increase throughout the entire water column, as implied in the derivation of (18). More realistic eddy viscosity models employ parameterizations such as $K=$ constant in the outer boundary layer (Kajura, 1967; Brevic, 1981), and show better agreement with observed phases of oscillatory flows. Results from a time-dependent eddy viscosity model can be found in Trowbridge et al. (1984) and demonstrate that the lowest order solution is almost insensitive to temporal variations in $K$.

¿From (19), it can be derived that $\frac{\delta^{-}}{\delta^{+}}=\frac{\sigma+f}{\sigma-f} \approx 5.2$ for the $\mathrm{M}_{2}$ constituent, while (16) suggests $\frac{\delta^{-}}{\delta^{+}}=\left[\frac{\sigma+f}{\sigma-f}\right]^{1 / 2} \approx 2.3$. With boundary layer heights $\delta^{+} \approx 8 \mathrm{~m}$ and $\delta^{-} \approx 40 \mathrm{~m}$ based on current amplitudes as discussed above, or with $\delta^{+} \approx 10 \mathrm{~m}$ and $\delta^{-} \approx 50 \mathrm{~m}$ if the veering of $\phi^{+}$and $\phi^{-}$is considered instead, the ratio $\frac{\delta^{-}}{\delta^{+}} \approx 5$ can be computed in good agreement with (19). Taking $\bar{u}_{*}=1.2 \mathrm{~cm} / \mathrm{s}$ derived 
from BASS data, the constant $c$ is appoximatley 0.39 and 0.48 for boundary layer heights based on current amplitudes and phase veering, respectively. The observed ratio $\frac{\delta^{-}}{\delta^{+}} \approx 5$ demonstrates that boundary layer thicknesses for $\mathbf{R}^{+}$and $\mathbf{R}^{-}$scale similarly to those quoted for planetary boundary layers and oscillatory flows, if the appropriate frequencies, $\sigma+f$ and $\sigma-f$, are used to describe the rotation of the respective rotary component. Since observations support (19) rather than (16), it is reasonable to conclude that the assumption of $K$ increasing linearly away from the seafloor, at least in some limited part of the boundary layer, is crucial to obtain a meaningful scaling of the boundary layer thickness. This statement is in partial agreement with earlier results from Soulsby (1990), which showed that $\left[\frac{\sigma+f}{\sigma-f}\right]^{1 / 2}<\frac{\delta^{-}}{\delta^{+}}<\frac{\sigma+f}{\sigma-f}$ for Celtic Sea data.

Results from analysis for the $\mathrm{S}_{2}$ and $\mathrm{N}_{2}$ semidiurnal constituents support the scaling arguments derived from the $\mathrm{M}_{2}$ currents. Since current amplitudes are small, it is difficult to estimate boundary layer thicknesses associated with the $\mathrm{N}_{2}$ and $\mathrm{S}_{2}$ constituents from magnitudes of their rotary components alone (Figures 22a and 23a). However, Figures 22b and 23b and c suggest that similar to the $M_{2}$ tide, phase angles $\phi^{+}$and $\phi^{-}$display strongest veering at heights less than approximately 10 and $50 \mathrm{~m}$, respectively. According to (19), boundary layer scaling heights vary depending on the frequency of the tidal constituent that is investigated. In the cases discussed above, all frequencies were in the semidiurnal range and therefore similar. Comparison of boundary layer thicknesses for the semidiurnal and diurnal components yields

$$
\frac{\delta_{d}^{ \pm}}{\delta_{s d}^{ \pm}}=\left|\frac{\sigma_{s d \pm} f}{\sigma_{d \pm} f}\right|
$$

where the indices $s d$ and $d$ refer to semidiurnal and diurnal, respectively. With $\sigma_{s d} \approx \sigma_{M_{2}}=1.41 \cdot 10^{-4} \frac{1}{\mathrm{~s}}$ and $\sigma_{d} \approx \sigma_{K_{1}}=0.73 \cdot 10^{-4} \frac{1}{\mathrm{~s}}$, the ratios $\frac{\delta_{f}^{+}}{\delta_{s d}^{+}} \approx 1.4$, and $\frac{\delta_{d}^{-}}{\delta_{s d}^{-}} \approx 2.1$ are obtained. Thus, estimated boundary layer heights for $\mathbf{R}^{+}$and $\mathbf{R}^{-}$are larger if the frequency is diurnal than if it is semidiurnal. Taking $\delta_{s d}^{-} \approx 40 \mathrm{~m}$ based on current amplitudes for the $\mathrm{M}_{2}$ tide, and $\delta_{s d}^{-} \approx 50 \mathrm{~m}$ based on the veering of $\phi^{-}$, the corresponding values for $\delta^{-}$of the $\mathrm{K}_{1}$ constituent according to (20) are approximately 84 and $105 \mathrm{~m}$, respectively. These values are larger than the actual 
water depth, suggesting that free-stream conditions of $R^{-}$and $\phi^{-}$are barely reached within the water column. Results for the $\mathrm{K}_{1}$ tide support these scaling arguments, as can be infered from the magnitudes $R^{-}$displayed in Figure 24a and from phase angles $\phi^{-}$shown in Figure 24c. The magnitudes of $R^{+}$for the $\mathrm{K}_{1}$ currents is too small to allow similar conclusions.

\section{III.2.4 The $\mathrm{M}_{2}$ Stress Ellipse}

The stress ellipse associated with the $M_{2}$ tide was investigated for two reasons. First, to determine whether the boundary layer height scaling introduced in the previous section applies similarly to the stress vector. Second, to compare bottom stresses derived from BASS data with those obtained from integration of the velocity defect in the bottom boundary layer. In order to draw conclusions about the vertical structure of the $M_{2}$ stresses, the rotary components of the stress ellipse needed to be found as a function of height. This was accomplished by integrating the momentum equations (13) from the sea floor to a given height $z$, where endpoints of the integration are between adjacent current meters. With the surface stresses being negligible in the semidiurnal frequency band, and with the $\mathrm{M}_{2}$ pressure forcing being barotropic, vertical integration of (13) yields

$$
\begin{aligned}
& \tau^{+}(z)=i(f+\sigma)\left[\int_{0}^{z} \boldsymbol{R}^{+} d z-\frac{z}{D} \int_{0}^{D} \boldsymbol{R}^{+} d z\right]+\left(1-\frac{z}{D}\right) \boldsymbol{\tau}_{b}^{+} \\
& \boldsymbol{\tau}^{-}(z)=i(f-\sigma)\left[\int_{0}^{z} \mathbf{R}^{-} d z-\frac{z}{D} \int_{0}^{D} \mathbf{R}^{-} d z\right]+\left(1-\frac{z}{D}\right) \boldsymbol{\tau}_{b}^{-} .
\end{aligned}
$$

where $z=0$ at the seafloor, $D$ is water depth, and $\tau_{b}^{ \pm}$are the rotary components of the bottom stress divided by density as obtained from tidal analysis of the hourly averaged stress estimates derived from best-fit logarithmic profiles to BASS velocity data according to (1) and (2). Tidal analysis reveals that the major contribution to the bottom stress occurs at the $\mathrm{M}_{2}$ frequency, with the major and minor axes of the stress ellipse, $\tau_{b_{M a j}}$ and $\tau_{b_{M i n}}$, being 1.90 and $-0.96 \mathrm{~cm}^{2} / \mathrm{s}^{2}$, respectively. Next largest 
contributions are from subtidal stresses and the $\mathrm{N}_{2}$ and $\mathrm{S}_{2}$ constituentsx, and lie between $22 \%$ and $26 \%$ of the $\mathrm{M}_{2}$ stress amplitudes.

Integrating (13) from bottom to surface, the pressure forcing is given by

$$
\begin{aligned}
& \mathbf{P}^{+}=-\frac{1}{D}\left[i(f+\sigma) \int_{0}^{D} \mathbf{R}^{+} d z+\tau_{b}^{+}\right], \\
& \mathbf{P}^{-}=-\frac{1}{D}\left[i(f-\sigma) \int_{0}^{D} \mathbf{R}^{-} d z+\boldsymbol{\tau}_{b}^{-}\right] .
\end{aligned}
$$

Results using (22) are in good agreement with those computed from free-stream values of the $\mathrm{M}_{2}$ currents according to (12). With $P_{M a j}=3.30 \mathrm{~cm}^{2} \mathrm{~s}^{-2}$ and $P_{\text {Min }}=0.19 \mathrm{~cm}^{2} \mathrm{~s}^{-2}$, differences between both methods are approximately 0.10 . $10^{-1} \mathrm{~cm}^{2} \mathrm{~s}^{-2}$ and $0.06 \cdot 10^{-1} \mathrm{~cm}^{2} \mathrm{~s}^{-2}$ for the major and minor axis, respectively. Since the $M_{2}$ pressure forcing is almost rectilinear, it is not surprising that relative changes of $P_{M i n}$ are larger than those of $P_{M a j}$. Inclinations for pressure ellipses computed from (12) and (22) differ by less than $2^{\circ}$.

The vertical structure of the rotary components, major and minor axes, phase, and inclination of the $\mathrm{M}_{2}$ stress ellipse are shown in Figure 25. Error bars are based on uncertainties of the $\mathrm{M}_{2}$ currents and are obtained from linear error propagation. Assumptions concerning uncertainties of phase and inclination angles are the same as described previously for the $\mathrm{M}_{2}$ current ellipse. Since measurement errors other than those of the BASS and VMCM compasses were not accounted for in the tidal current analysis, it follows that they are also not considered in the analysis of the stress vector. However, it has been mentioned before that current ellipses are insensitive to random errors corresponding to the uncertainty of the VMCMs. Since stress ellipses are computed from vertical integration of the tidal currents, this conclusion similarly applies to the results discussed in this paragraph. The contribution of uncertainties for $u_{*}$ from the logarithmic fit to bottom stresses at the $M_{2}$ frequency is not known and did not enter the error analysis.

It can be seen from Figures 25a and d that stress amplitudes approach zero in the flow interior at heights larger than approximately $40 \mathrm{~m}$. Because uncertainties of 
$\tau^{+}$and $\tau^{-}$increase as the surface is approached, it is difficult to infer boundary layer thicknesses from the magnitudes of the rotary components. Correspondingly, associated phase angles $\phi_{\tau}^{+}$and $\phi_{\tau}^{-}$are less determined with increasing distance from the seafloor. Nevertheless, their vertical structure strongly supports earlier results for the $M_{2}$ currents, suggesting that phase veering is strongest at heights less than approximately $10 \mathrm{~m}$ and $50 \mathrm{~m}$, respectively.

In order to compare bottom stress estimates from BASS data to those obtained from integration of the velocity defect, a height $z_{\infty}$ needs to be specified at which the free-stream conditions are reached. Due to the temporal variability of the subtidal flow, whose vertical structure depends on processes such as surface winds and horizontal density gradients, it is impossible to define free-stream conditions and corresponding heights for the total flow. Therefore, only bottom stresses in the $\mathrm{M}_{2}$ frequency band were used for this comparison. If a height $\mathrm{g} z_{\infty}$ is chosen such that $\mathrm{M}_{2}$ currents approach their free-stream values in the region above, velocity shears are negligible at heights larger than $z_{\infty}$. According to (13), rotary components of the bottom stress can then be computed from

$$
\begin{aligned}
& \boldsymbol{\tau}^{+}(z)=-i(f+\sigma) \int_{z}^{z_{\infty}} \mathbf{R}^{+} d z-\left(z_{\infty}-z\right) \mathbf{P}^{+} \\
& \boldsymbol{\tau}^{-}(z)=-i(f-\sigma) \int_{z}^{z_{\infty}} \mathbf{R}^{-} d z-\left(z_{\infty}-z\right) \mathbf{P}^{-}
\end{aligned}
$$

if the lower limit of integration $z$ is set to zero. Taking $\mathbf{P}^{ \pm}$as the pressure forcing derived from (12), no prior assumptions concerning the magnitude or orientation of the bottom stress vector are made.

Figure 25 shows that $z_{\infty} \approx 35 \mathrm{~m}$ is a reasonable value to describe the transition to free-stream conditions. Results from (23) are shown in Figure 26, where dotted lines correspond to stress values previously obtained from (14). Magnitudes $\tau_{b}^{+}$and $\tau_{b}^{-}$as well as $\tau_{b_{M a j}}$ and $\tau_{b_{M i n}}$ computed from tidal analysis of BASS stress estimates and from the velocity defect show reasonable agreement. Differences of $\tau_{b}^{+}, \tau_{b}^{-}$, $\tau_{b_{M a j}}$ and $\tau_{b_{M i n}}$ obtained from both methods are less than $0.09,0.38,0.29$ and 
$0.47 \mathrm{~cm}^{2} / \mathrm{s}^{2}$, respectively. Inclinations of the stress ellipse at $z=0$ are also similar and deviate by approximately $8^{\circ}$. Phases at the seafloor are off by $38^{\circ}$. The observation that inclination angles at some height above the bottom display significant differences depending on the method used is not surprising. An explanation for this behavior is given by the fact that the phase angles $\phi_{\tau}^{+}$and $\phi_{\tau}^{-}$are less determined the further the distance from the bottom due to decreases in the magnitudes $\tau^{+}$and $\tau^{-}$. Thus, relatively small deviations in stress amplitudes obtained from both methods may result in large discrepancies between associated phase angles. In summary, the reasonably close agreement of bottom stress amplitudes and inclination angles computed from two entirely different methods supports the stress estimates derived from BASS data.

\section{III.2.5 Boundary Layer Transports}

In the previous section, it was shown that bottom stresses obtained from leastsquares logarithmic fits to BASS velocity data and from integration of the velocity defect in the boundary layer show reasonable agreement at the frequency of the $M_{2}$ tide. This result is partly due to the fact that a well-defined interior flow regime exists, allowing the velocity defect to be determined with considerable accuracy. It also implies that bottom-stress estimates based on BASS data are accurate to within $20 \%$ and $27 \%$ for the clockwise and anticlockwise component of the stress ellipse, respectively. Both statements suggest that boundary layer transports may be computed from integration of the velocity defect as well as from bottom stress estimates obtained from BASS velocity measurements, according to

$$
\begin{aligned}
& \int_{0}^{z_{\infty}^{+}}\left(\mathbf{R}^{+}-\mathbf{R}_{\infty}^{+}\right) d z=\frac{i \boldsymbol{\tau}_{b}^{+}}{f+\sigma}, \\
& \int_{0}^{z_{\infty}^{-}}\left(\mathbf{R}^{-}-\mathbf{R}_{\infty}^{-}\right) d z=\frac{i \boldsymbol{\tau}_{b}^{-}}{f-\sigma},
\end{aligned}
$$

In (24), $z_{\infty}^{+}$and $z_{\infty}^{-}$denote the heights at which $\mathbf{R}^{+}$and $\mathbf{R}^{-}$reach their free-stream values, respectively. $\tau_{b}^{ \pm}$are obtained from tidal analysis of the stress values derived from BASS data according to (1) and (2). Figure 27 depicts results from the left- and 
right-hand sides of (24) for their magnitudes $T^{+}$and $T^{-}$, and corresponding phase angles $\phi_{T}^{+}$and $\phi_{T}^{-}$. The left-hand sides of (24) are depicted as solid lines, where the upper limit of integration has been varied from zero to the total water depth $D$. The right-hand sides are presented as dashed lines. Current measurements taken at height $30.5 \mathrm{~m}$ above the bottom were not used to integrate the velocity defect, since they are believed to over-estimate the actual current speeds (Figures 18 and 20). Including these velocities would particularly affect the vertical structure of $\int_{0}^{z}\left(\mathbf{R}^{+}-\mathbf{R}_{\infty}^{+}\right) d z$ in the upper part of the water column, since magnitudes $R^{+}$are small and their vertical integral is sensitive to measurement errors. The profile of $\int_{0}^{z}\left(\mathbf{R}^{-}-\mathbf{R}_{\infty}^{-}\right) d z$ proved to be insensitive to inclusion of velocities measured at $30.5 \mathrm{~m}$ above the bottom, because magnitudes $R^{-}$are large. Error bars of the left-hand sides of (24) were obtained from linear error propagation, with the lower limit of the uncertainties for $\phi_{T}^{+}$and $\phi_{T}$ being set by VMCM and BASS compass errors. Uncertainties of bottom stresses from the logarithmic fit are not known at the $\mathrm{M}_{2}$ frequency, so that no error estimates can be given for the right-hand sides of (24).

It can be seen from Figure 27 that integrals $\int_{0}^{z}\left(\mathbf{R}^{+}-\mathbf{R}_{\infty}^{+}\right) d z$ and $\int_{0}^{z}\left(\mathbf{R}^{-}-\right.$ $\left.\mathbf{R}_{\infty}^{-}\right) d z$ approach constant values at some height away from the sea floor. Defining boundary layer heights $\delta^{+}$and $\delta^{-}$such that the magnitudes of the integrated velocity defects have approximately reached approximately $95 \%$ of their free-stream values yields $\delta^{+} \approx 8 \mathrm{~m}$ and $\delta^{-} \approx 40 \mathrm{~m}$. These values are in agreement with earlier results for boundary layer thicknesses of the rotary components of the $\mathrm{M}_{2}$ current ellipse. Near the surface, magnitudes $T^{+}$and $T^{-}$computed from velocity defects are $\approx 0.15 \mathrm{~m}^{2} \mathrm{~s}^{-1}$ and $\approx 4.60 \mathrm{~m}^{2} \mathrm{~s}^{-1}$, respectively, while corresponding magnitudes based on bottom stress estimates are $\approx 0.20 \mathrm{~m}^{2} \mathrm{~s}^{-1}$ and $\approx 3.15 \mathrm{~m}^{2} \mathrm{~s}^{-1}$, respectively. Phases $\phi_{T}^{-}$obtained from the right and left hand side of $(24 \mathrm{~b})$ differ by roughly $10^{\circ}$. Phase angles $\phi_{T}^{+}$show less good agreement, their difference being about $50^{\circ}$. This behavior is explained by the fact that corresponding magnitudes $T^{+}$are small. The difference $\Delta T^{-} \approx 1.45 \mathrm{~m}^{2} \mathrm{~s}^{-1}$ between the right and left-hand side of (24b) may suggest that near surface current amplitudes have not reached their free stream values over the given water depth, but exceed these values by approximately $2 \mathrm{~cm} / \mathrm{s}$. This explanation is supported by the vertical structure of $T^{-}$(Figure $27 \mathrm{~b}$ ), which, 
unlike $T^{+}$, does not display an oscillation around an asymptotic value, as suggested by the analytical solutions (15) and (18). The behaviour of $T^{-}$indicates that due to $\delta^{-}>>\delta^{+}$, the asymptotic approach of a constant value $R_{\infty}^{-}$is completed to a lesser degree than that of $R_{\infty}^{+}$. Another reason for the observed difference between boundary layer transports based on bottom stress estimates and velocity defects may be associated with insufficient data coverage in the lower part of the water column. In particular, the data gap between 12 and $30.5 \mathrm{~m}$ above the bottom could account for considerable inaccuracies in the vertical integration of measured velocities.

\section{III.2.6 Eddy Viscosity Profiles}

In III.2.3, a meaningful scaling of tidal boundary layer heights was shown to depend on the assumption that a constant stress layer exists in the vicinity of the sea floor, thus implying that (17) is valid near the boundary. In order to investigate the extent to which this statement is true, eddy viscosities were computed from measured velocity shears and stress distributions in the entire water column. Stresses were obtained from the vertical integral of the horizontal momentum equations, which for the total flow are given by

$$
\begin{aligned}
& \frac{\partial u}{\partial t}-f v=-\frac{1}{\rho_{0}} \frac{\partial p}{\partial x}+\frac{1}{\rho_{0}} \frac{\partial \tau^{x}}{\partial z} \\
& \frac{\partial v}{\partial t}+f u=-\frac{1}{\rho_{0}} \frac{\partial p}{\partial y}+\frac{1}{\rho_{0}} \frac{\partial \tau^{y}}{\partial z} .
\end{aligned}
$$

In (25), $\mathrm{x}$ is along-bank gand $\mathrm{y}$ is across-bank distance in the off-bank direction, where along-bank is at $-148^{\circ}$ from east and perpendicular to the $M_{2}$ pressure forcing, $\rho_{0}$ is mean density, $p$ is pressure, and $\tau^{x}$ and $\tau^{y}$ are the along- and acrossbank components of the stress vector, respectively. With total flow magnitudes being similar to current amplitudes of the $\mathrm{M}_{2}$ tide, Rossby numbers are small as shown in II.2.2, and advective terms are negligible at lowest order. CTD cross-sections taken in February and March 1995 reveal that horizontal density gradients at ST1 were of order $0.05-0.1 \sigma_{t}$ units over $20-\mathrm{km}$ distance in the offbank direction, corresponding 
to a thermal wind of $\frac{\Delta u}{\Delta z} \approx(2.5-5) \cdot 10^{-4} \mathrm{~s}^{-1}$. With the water depth being $76 \mathrm{~m}$, the total velocity shear between bottom and surface caused by the thermal wind is approximately $(2-4) \mathrm{cm} / \mathrm{s}$, which is small compared to the measured current speeds. Thus, $(25 \mathrm{a}, \mathrm{b})$ are to lowest order barotropic.

Under these assumptions, the vertical integral of (25) yields

$$
\begin{aligned}
& \frac{1}{\rho_{0}} \tau^{x}(z)=\int_{0}^{z}\left(\frac{\partial u}{\partial t}-f v\right) d z-\frac{z}{D} \int_{0}^{D}\left(\frac{\partial u}{\partial t}-f v\right) d z+\left(1-\frac{z}{D}\right) \frac{\tau_{b}^{x}}{\rho_{0}}+\frac{z}{D} \frac{\tau_{w}^{x}}{\rho_{0}}, \\
& \frac{1}{\rho_{0}} \tau^{y}(z)=\int_{0}^{z}\left(\frac{\partial v}{\partial t}+f u\right) d z-\frac{z}{D} \int_{0}^{D}\left(\frac{\partial v}{\partial t}+f u\right) d z+\left(1-\frac{z}{D}\right) \frac{\tau_{b}^{y}}{\rho_{0}}+\frac{z}{D} \frac{\tau_{w}^{y}}{\rho_{0}},
\end{aligned}
$$

where $\tau_{b}^{x}$ and $\tau_{b}^{y}$ are the along- and cross-bank components of the bottom stress, and $\tau_{w}^{x}$ and $\tau_{w}^{y}$ the corresponding components of the wind stress, respectively. Bottom-stress estimates were taken from BASS data, and wind stresses were computed from IMET wind measurements using the Large and Pond algorithm (Large and Pond, 1981). Endpoints of the integration are between adjacent current meters. According to $\frac{1}{\rho_{0}}\left(\tau^{x}, \tau^{y}\right)=K \frac{\partial(u, v)}{\partial z}$, the eddy viscosity is given by

$$
K=\frac{1}{\rho_{0}} \frac{\tau}{\left[\left(\frac{\partial u}{\partial z}\right)^{2}+\left(\frac{\partial v}{\partial z}\right)^{2}\right]^{1 / 2}}
$$

where $\tau$ is the magnitude of the stress vector. Application of (27) yields the eddy viscosity profiles shown in Figure 28. Figure 28a displays the hourly variation in $K$ during one $\mathrm{M}_{2}$ tidal cycle, and Figure 28b represents the vertical structure of the time mean $K$. The dashed line in Figure 28b depicts the slope $\kappa \bar{u}_{*} z$, with $\bar{u}_{*}$ being the time-mean friction velocity of $1.2 \mathrm{~cm} / \mathrm{s}$. Figure $28 \mathrm{c}$ is the same as Figure 28a but with the vertical axis being restricted to the bottom $5 \mathrm{~m}$. Error bars were obtained from linear error propagation, where the $95 \%$ confidence intervals of $u_{*}$ have been used to compute uncertainties of the bottom stress. It can be seen from Figures $28 \mathrm{a}$ and $\mathrm{b}$ that the vertical structure of $K$ can best be described as parabolical. Eddy viscosities approach zero near the surface and bottom, where turbulent motion is restricted by the upper and 
lower boundaries. Computation of $K$ from $\mathrm{M}_{2}$ currents alone yields results almost identical to those shown in Figure 28 for the lowest $25-30 \mathrm{~m}$. Above these heights, tidal current shears are small (Figure 20), and turbulence is largely sustained by velocity shears in the subtidal frequency range. Uncertainties of $K$ are greatest in the middle of the water column, which represents a region of small current shear located between the surface and bottom turbulent layers.

Comparison of eddy viscosity profiles to the slope $\kappa \bar{u}_{*} z$ in Figures $28 \mathrm{~b}$ and c suggests that (17) holds only in the near-vicinity of the sea floor, i.e., at heights smaller than 1.5-2.0 m above the bottom. On the other hand, the thickness of the logarithmic layer extends to greater heights as shown in Figure 29. Velocity profiles shown in the figure are for total current speed and correspond to those depicted in Figure 18. Dashed lines are results from a least-squares logarithmic fit to current speeds measured at pods 1-4. Visible differences between measured speeds and best-fit logarithmic profiles occur between pods 4 and 5, indicating the thickness of the logarithmic layer to be of order $2.5-4.5 \mathrm{~m}$. At these heights, time-averaged deviations from the bestfit logarithmic profiles are less than $5.5 \%$ and $3 \%$ for $z<4.5$ and $z<2.5 \mathrm{~m}$, respectively. Based on these results, the vertical extent to which (17) is valid is, at most, one-half the height of the logarithmic layer. This behavior is in agreement with earlier results from Grant et al. (1986). Their conclusions, based on boundary layer equations and scaling arguments, state that the logarithmic velocity profile in planetary boundary layers is accurate to a greater height than the approximation of a constant stress layer. Similar evidence can be found in Figure 25, which suggests that over the tripod height, stress amplitudes have decreased by more than $20 \%$, and inclination as well as gphase angles have changed by approximately $15^{\circ}$. Since (17) is derived under the assumptions that stresses are constant and the velocity profile is logarithmic, it follows that $K=\kappa \bar{u}_{*} z$ can hold only at heights where both assumptions are valid to lowest order.

In summary, two conclusions can be drawn from the eddy viscosity profiles shown in Figure 28. First, $K$ increases with height between the seafloor and approximately $40 \mathrm{~m}$ above the bottom, and does not approach a constant value anywhere 
in the bottom boundary layer. Therefore, boundary layer height scaling according to $\delta^{ \pm} \approx\left|\frac{K}{\sigma \pm f}\right|^{1 / 2}$ is not realistic, a statement that is supported by observational evidence. Second, $K=\kappa \bar{u}_{*} z$ is valid in some limited region of the logarithmic layer, supporting scaling arguments that $\delta^{ \pm} \approx \frac{\kappa \bar{u}_{*}}{|\sigma \pm f|}$ which is in better agreement with the observations.

\section{III.3 Numerical Modeling}

\section{III.3.1 Model Description}

\section{III.3.1.1 The K-Profile Model}

The $K$-profile model makes use of prognostic equations for the vertical structure of the mixing coefficient, $K$. It is a one-dimensional finite-difference code that integrates the linear momentum equations forward in time, and solves for velocities and eddy viscosities after each time step. With $\frac{1}{\rho_{0}}\left(\tau^{x}, \tau^{y}\right)=K\left(\frac{\partial u}{\partial z}, \frac{\partial v}{\partial z}\right)$, the linear momentum equations for a homogeneous flow are given by

$$
\begin{aligned}
\frac{\partial u}{\partial t}-f v & =-\frac{1}{\rho_{0}} \frac{\partial p}{\partial x}+\frac{\partial}{\partial z}\left(K \frac{\partial u}{\partial z}\right), \\
\frac{\partial v}{\partial t}-f u & =-\frac{1}{\rho_{0}} \frac{\partial p}{\partial y}+\frac{\partial}{\partial z}\left(K \frac{\partial v}{\partial z}\right), \\
g & =-\frac{1}{\rho_{0}} \frac{\partial p}{\partial z} .
\end{aligned}
$$

Equation (28c) states the hydrostatic balance and does not enter the numerical model.

A parameterization for $K$ is found based on (17), which is valid in some limited region near the boundary. Since it is the structure of the tidally driven bottom boundary layer that is investigated here, a simple two-layer model is believed sufficient, and 
$K$ is specified according to

$$
K=\left\{\begin{array}{lll}
\kappa \bar{u}_{*} z & \text { for } z \leq l \\
\kappa \bar{u}_{*} l & \text { for } z>l
\end{array}\right.
$$

In (29), the time-mean friction velocity $\bar{u}_{*}$ during one tidal cycle is taken as a characteristic value for turbulent fluctuations near the boundary. This is in agreement with analytical results from Trowbridge et al. (1984), suggesting that temporal variations in $K$ for a parameterization similar to (29) do not affect the flow structure to lowest order. The sublayer height to which $K=\kappa \bar{u}_{*} z$ may be taken as $l=A \delta$, where the constant $A$ is empirical, and $\delta$ is a scaling height for the total boundary layer-thickness. According to results for planetary boundary layers and rectilinear flows with frequency $\omega$ quoted earlier, appropriate scaling heights $\delta$ for such flows are $\frac{\kappa u_{*}}{f}$ and $\frac{\kappa \hat{u}_{*}}{\omega}$, respectively. In the present case, a meaningful parameterization may be given by $\delta=\frac{\kappa \bar{u}_{*}}{\sigma-f}$, since it is the clockwise rotary component that dominates the vertical structure of the bottom boundary layer. The empirical constant $A$ is not well known, and its specification is somewhat arbitrary. With (29a) being derived from the logarithmic law of the wall, it is to be expected that reasonable values for $l$ are of the order of the logarithmic layer thickness known to be $\approx 0.1 \delta$ for steady and rectilinear flows (Clauser, 1956; Grant, 1977; Grant et al., 1986). Trowbridge et al. (1984) suggest $l=\frac{1}{6} \delta$ for rectilinear flows, since model results using this parameterization are in good agreement with laboratory experiments carried out by Jonsson and Carlsen (1976). Similar scaling was applied by Beardsley et al. (1995) in the investigation of rectilinear tidal currents in the Amazon River. However, no extensive study is known in which the sensitivity of model results to alterations in $A$ has been investigated.

Since the model is homogeneous and surface heat fluxes are neglected, (28) and (29) fully describe the involved physics. In the absence of wind stress, surface and bottom boundary conditions can be expressed as 


$$
\begin{array}{cc}
\frac{\partial u}{\partial z}=\frac{\partial v}{\partial z}=0 & \text { at } z=D \\
K\left(\frac{\partial u}{\partial z}, \frac{\partial v}{\partial z}\right)=\frac{1}{\rho_{0}}\left(\tau_{b}^{x}, \tau_{b}^{y}\right) & \text { at } z=z_{0} .
\end{array}
$$

Bottom stress is computed according to

$$
\frac{1}{\rho_{0}}\left(\tau_{b}^{x}, \tau_{b}^{y}\right)=c_{D} \cdot \sqrt{u^{2}+v^{2}}(u, v),
$$

where

$$
c_{D}=\left[\frac{\kappa}{\ln \left(\frac{z_{b}}{z_{0}}\right)}\right]^{2} .
$$

In (32), $z_{b}$ is the height of the lowest grid point at which velocities are computed. With $z_{b}=0.25 \mathrm{~m}$ and $z_{0}=0.7 \mathrm{~mm}$, the drag coefficient is given by $c_{D}=4.63 \cdot 10^{-3}$. Bottom roughness was specified based on results from least-squares fits of $U^{2}$ to $u_{*}^{2}$ discussed in II.4.2. Bottom stress is computed at each time step $n$ in order to provide the boundary condition at $z=z_{0}$ for $n+1$. Friction velocities are obtained from $u_{*}=\sqrt{\frac{\tau_{b}}{\rho_{0}}}$. After the completion of each tidal cycle, friction velocities are averaged, and mixing coefficients for the following cycle are computed according to (29). The vertical grid spacing used in all model runs is $\Delta z=0.5 \mathrm{~m}$, and time steps are $\Delta t=\frac{T_{M_{2}}}{500}$, where $T_{M_{2}}=12.42$ hours is the period of one $\mathrm{M}_{2}$ tidal cycle. The model uses a control volume approach, with velocities being computed in the center of the constant volume, and eddy viscosities being evaluated at the upper and lower surfaces. Vertical diffusion is incorporated applying the Crank-Nicholson time-space integration scheme.

The model is forced by the pressure gradient $\frac{1}{\rho_{0}}\left(\frac{\partial p}{\partial x}, \frac{\partial p}{\partial y}\right)$, which is specified as a function of time. Initial conditions are those of no flow, $u=v=0$, and $K=1 \cdot 10^{-6} \mathrm{~m}^{2} / \mathrm{s}$ corresponding to the molecular viscosity of water. In order to keep inertial oscillations as small as possible, the pressure forcing is linearly increased to its final amplitude during the period $2 \cdot \frac{2 \pi}{f}$. For the investigation of $M_{2}$ currents, integration over 12 tidal cycles is necessary to bring solutions to a steady state. 


\section{III.3.1.2 The Mellor-Yamada Level 2.5 Closure}

A one-dimensional version of the Blumberg and Mellor (1987) model was also used to compare model results with actual data. The numerical code incorporates Mellor and Yamada's $(1974,1982)$ level 2.5 turbulence closure scheme (MY 2.5). With assumptions concerning linearity and homogeneity of the flow being the same as in III.3.1.1, momentum equations are given by (28). The MY 2.5 makes use of two additional equations, the turbulent kinetic energy and the turbulent macroscale equations, to provide a parameterization of vertical mixing. For a one-dimensional, homogeneous flow, these equations can be written as

$$
\begin{aligned}
\frac{\partial q^{2}}{\partial t} & =\frac{\partial}{\partial z}\left(K_{q} \frac{\partial q^{2}}{\partial z}\right)+P_{S}-\epsilon, \\
\frac{\partial}{\partial t}\left(q^{2} l_{m}\right) & =\frac{\partial}{\partial z}\left(K_{q} \frac{\partial\left(q^{2} l_{m}\right)}{\partial z}\right)+l_{m} E_{1}\left(P_{S}-\epsilon W\right),
\end{aligned}
$$

respectively. In (33) and (34), $q^{2}$ is the turbulent kinetic energy, $K_{q}$ the eddy diffusion coefficient of turbulent kinetic energy, $l_{m}$ the turbulent macroscale, $P_{S}$ the shear production of turbulent energy defined as

$$
P_{S}=K\left[\left(\frac{\partial u}{\partial z}\right)^{2}+\left(\frac{\partial v}{\partial z}\right)^{2}\right],
$$

and $\epsilon$ the turbulent energy dissipation given by

$$
\epsilon=\frac{q^{3}}{B_{1} l_{m}} .
$$

$\mathrm{W}$ is a wall proximity function described by

$$
W=1+E_{2}\left(\frac{l_{m}}{\kappa z}\right)^{2}+E_{3}\left(\frac{l_{m}}{\kappa(D-z)}\right)^{2} .
$$

The constants $E_{1}$ in (34), $\quad B_{1}$ in (36), and $E_{2}$ and $E_{3}$ in (37) are empirical. Equations (28), (33), and (34) are closed by defining

$$
K=l_{m} S, K_{q}=l_{m} S_{q}
$$


where $S$ and $S_{q}$ are stability functions satisfying

$$
\begin{aligned}
S\left(6 A_{1} A_{2} G_{M}\right) & =A_{2} \\
S\left(1+6 A_{1}^{2} G_{M}\right) & =A_{1}\left(1-3 C_{1}\right) \\
S_{q} & =0.20
\end{aligned}
$$

and where

$$
G_{M}=\frac{l_{m}^{2}}{q^{2}}\left[\left(\frac{\partial u}{\partial z}\right)^{2}+\left(\frac{\partial v}{\partial z}\right)^{2}\right]
$$

With the empirical constants

$$
\left(A_{1}, A_{2}, B_{1}, C_{1}, E_{1}, E_{2}, E_{3},\right)=(0.92,0.74,16.6,0.08,1.33,0.25)
$$

derived from laboratory experiments (Mellor and Yamada, 1974, 1982), $S$ can be determined according to (39) as a function of vertical flow shear.

In the absence of momentum and heat flux at the surface and bottom, boundary conditions can be expressed as

$$
\left.\begin{array}{rl}
\frac{\partial u}{\partial z} & =\frac{\partial v}{\partial z}=0 \\
q^{2} & =q^{2} l_{m}=0
\end{array}\right\} \quad \begin{aligned}
& \text { at } z=D \\
&
\end{aligned}
$$


and

$$
\left.\begin{array}{rl}
K\left(\frac{\partial u}{\partial z}, \frac{\partial v}{\partial z}\right) & =\frac{1}{\rho_{0}}\left(\tau_{b}^{x}, \tau_{b}^{y}\right) \\
q^{2} & =B_{1}^{2 / 3} U^{* 2} \\
q^{2} l_{m} & =0
\end{array}\right\} \quad \text { at } z=z_{0}
$$

Bottom stress is computed according to (31) and (32).

The resolution of the vertical grid $\Delta z$, the size of the time step $\Delta t$, and the specification of the bottom roughness $z_{0}$ are the same as in III.3.1.1. Similar to the $K$-profile model, the numerical code uses the control volume approach to solve for the variables $u, v, K, K_{q}, q^{2}$, and $l_{m}$. Velocities are computed at the center of the control volume, while mixing coefficients, turbulent kinetic energy, and turbulent macroscale are evaluated at the upper and lower surfaces. Pressure forcing and bottom roughness are the same as for the $K$-profile model.

\section{III.3.2 Model Performance}

\section{III.3.2.1 Performance of the K-Profile Model}

According to (29), the performance of the $K$-profile model is dependent on specifications concerning the parameterization $l=A \delta$, where $\delta$ is given by $\frac{\kappa u_{*}}{f}$ and $\frac{\kappa \hat{u}_{*}}{\omega}$ for steady planetary boundary layers and rectilinear oscillating flows, respectively. However, the $\mathrm{M}_{2}$ tidal current is subject to the Earth's rotation as well as to semidiurnal pressure forcing, and can therefore neither be described as rectilinear and oscillating, nor as steady and rotating. An alternative scaling for the total boundary layer thickness $\delta$ can be derived if the fact $R^{-} \approx 5 R^{+}$is taken into account (Figure 20a). With values of $R^{-}$significantly exceeding those of $R^{+}$at all depths, it is reasonable to suspect that the bottom boundary layer structure is dominated by the clockwise rotating component. This statement is supported by profiles of current amplitudes 
shown in Figure 20d, suggesting that both $U_{M a j}$ and $U_{M i n}$ approach free-stream values at heights similar to those where magnitudes $R^{-}$reach some constant magnitude. An appropriate scaling for the total boundary layer height might therefore be given by $\delta=\frac{\kappa \bar{u}_{*}}{\sigma-f}$, where the maximum amplitude of friction velocity during one tidal cycle, $\hat{u}_{*}$, has been replaced by its mean value $\bar{u}_{*}$. Taking $A=\frac{1}{6}$ as suggested by Trowbridge et al. (1984) yields the sublayer height $l=\frac{1}{6} \cdot \frac{\kappa \bar{u}_{0}}{\sigma-f}$, which will be used as a first parameterization to examine the performance of the $K$-profile model. Other parameterizations based on different values $A$ will also be investigated.

The numerical model was forced by a semidiurnal pressure gradient derived from the $\mathrm{M}_{2}$ currents according to (22). No mean pressure gradient was included in the forcing, since the weak subtidal flow at ST1 is not expected to affect the vertical structure of the tidal velocities. Based on drag coefficients discussed in II.4, the bottom roughness was taken as $z_{0}=0.7 \mathrm{~mm}$.

Figure 30 shows model results for the vertical structure of the current ellipse using $l=\frac{1}{6} \cdot \frac{\kappa \bar{u}_{*}}{\sigma-f}$. Circles represent results from tidal analysis of BASS and VMCM data and are the same as Figure 20. Dotted lines depict uncertainties associated with the data. For easier comparison of observations and model results, values for phase angles $\phi^{+}, \phi^{-}$, and $\phi$, and inclination $\theta$ have been replaced by their relative veering away from the surface. It can be seen from Figures $30 \mathrm{a}$ and $\mathrm{d}$ that observed magnitudes $R^{+}$and $R^{-}$, and current amplitudes $U_{M a j}$ and $U_{M i n}$ are predicted well in the upper part of the water column, while the model over-estimates measured current speeds at heights less than $\approx 25 \mathrm{~m}$ above the bottom. Phases of the clockwise component (Figure 30c) as well as phases of the current ellipse (Figure 30e) obtained from the model might be taken to under-predict the actual veering of these angles according to observations, but are overall within the limits of data uncertainties. Model results for inclination display anticlockwise rotation as the bottom is approached, while data suggest clockwise rotation of the major axis (Figure 30d). However, total changes in inclination predicted by the model are small $\left(\approx 3^{\circ}\right)$ and mostly within the margins of error bars associated with observations. 
Eddy viscosity profiles derived from numerical results are shown in Figure 31. In this figure, circles correspond to mixing coefficients computed from actual data, and dotted lines are the associated uncertainties. Comparison of observational and model results demonstrates that eddy viscosities predicted by the model are large compared to those computed from velocity measurements. Already at heights greater than $1.5 \mathrm{~m}$, numerical values for $K$ exceed those based on measured current shears, and they are about three times as large in most of the outer boundary layer. Vertical averages of time-mean mixing coefficients between the seafloor and $25 \mathrm{~m}$ above the bottom are 640 and $162 \mathrm{~cm}^{2} / \mathrm{s}$ for model results and observational data, respectively. This discrepancy is explained by the fact that according to $l=\frac{1}{6} \cdot \frac{\kappa \bar{u}_{*}}{\sigma-f}$, the sublayer thickness is approximately $20 \mathrm{~m}$, a value too large for numerical mixing coefficients to be similar to those computed from actual data. As a consequence of extending the region where $K=\kappa \bar{u}_{*} z$ to such a great height, model results significantly overestimate the logarithmic layer thickness observed in measured velocity profiles. Using model speeds between 0.25 and $2.75 \mathrm{~m}$ above the bottom to compute the logarithmic fit, numerical data predict less than a $1 \%$ deviation from the best-fit profile at heights smaller than $8.5 \mathrm{~m}$, and less than $5 \%$ everywhere else in the water column. This is in contrast with results from observations indicating that already at $\approx 4.5 \mathrm{~m}$ above the bottom, measured current speeds deviate by $\approx 5 \%$ from their logarithmic values, and that deviations are of order $30 \%$ near the free surface. From these results it must be concluded that the parameterization $l=\frac{1}{6} \cdot \frac{\kappa \bar{u}_{*}}{\sigma-f}$ leads to over-estimation of the observed logarithmic layer thickness as well as to over-prediction of actual eddy viscosity magnitudes.

Ellipse parameters for the model stresses are shown as a function of height in Figure 32, with circles and dotted lines representing data results according to Figure 25. Numerical predictions for bottom stresses are within $30 \%$ of those obtained from BASS data, with the model results over-estimating stress values derived from best-fit logarithmic profiles to measured current speeds. Time-averaged friction velocities during one tidal cycle are $\bar{u}_{*}=1.35 \mathrm{~cm} / \mathrm{s}$, as opposed to $\bar{u}_{*} \approx 1.2 \mathrm{~cm} / \mathrm{s}$ based on observations. It can be seen from Figure 32 that the model fails to reproduce the observed region of small stress magnitudes at heights larger than $\approx 40 \mathrm{~m}$. Reasons for this behavior 
are that numerical eddy viscosities at these heights are at least three times as large as those obtained from observations, and that some velocity shear, although small, is present in the model results for the upper part of the water column (Figure 30).

Boundary layer transports computed from numerical data are shown in Figure 33. Solid and dashed lines depicted in the figure correspond to similar lines in Figure 27, but are based on model results instead of observations. Circles and error margins are the same as Figure 27, and straight solid lines display boundary layer transports computed from bottom stress estimates using BASS data. The velocity defect was integrated taking the surface values of $\mathbf{R}^{ \pm}$, denoted as $\mathbf{R}_{S}^{ \pm}$, as $\mathbf{R}_{\infty}^{ \pm}$. It is important to realize that these values are not representative of the free stream, since numerical results do not exactly reach free-stream conditions in the given water depth. Closer investigation shows that magnitudes $R_{S}^{ \pm}$are within $5 \%$ of those given for $R_{\infty}^{ \pm}$by (22), while phases $\phi_{S}^{ \pm}$differ up to $8^{\circ}$ from corresponding values $\phi_{\infty}^{ \pm}$. Integrated over the entire water depth, these differences are large enough to explain the alleged imbalance between the left and right-hand sides of (24) evident in the figure. Since free-stream conditions are not reached inside the water column, it is the integral $\int_{0}^{D}\left(\mathbf{R}^{ \pm}-\mathbf{R}_{s}^{ \pm}\right) d z$ that correctly describes the total velocity defect predicted by the model. It can be seen from Figure 33 that boundary layer transports associated with the clockwise rotating component are significantly smaller than those obtained from observational data. The under-estimation of observed boundary layer transports is very likely due to the overprediction of actual current speeds in the lower part of the water column (Figure 30a,d). This behavior can be attributed to the scaling of the sublayer height, $l$, which, as described earlier, represents too large a thickness for $K$ to be specified according to (29). Because of the large value of $l$, numerical eddy viscosities are significantly greater than those derived from measured velocity shears, and the effects of turbulence are overemphasized. Thus, the model produces too much mixing and consequently exaggerates the convex shape of current amplitudes versus height in the bottom boundary layer.

It is therefore reasonable to test a different scaling for the thickness of the logarithmic layer, where parameterization of the sublayer height according to $l=A \delta$ requires specification of the constant $A$ as well as the total boundary layer thickness $\delta$. Al- 
though limited observational evidence for steady planetary boundary layers and experimental as well as numerical investigation of rectilinear flows with fequency $\omega$ suggest $\delta$ to be of order $\frac{\kappa u_{*}}{f}$ and $\frac{\kappa \hat{u}_{*}}{\omega}$, respectively, it is important to realize that the above expressions represent no more than scale heights, and cannot be expected to exactly predict the location at which free-stream conditions are reached. Similar conclusions apply to the present case of a rotary tide, especially since it was shown in III.2.3 that $\delta^{-} \approx 0.39 \frac{\kappa \bar{u}_{*}}{(\sigma-f)}$ based on current amplitudes $R^{-}$, as opposed to $\delta=\frac{\kappa \bar{u}_{*}}{(\sigma-f)}$ used in (29). With $\delta \approx \delta^{-}$, application of $l=\frac{1}{6} \delta=\frac{1}{6} \cdot 0.39 \frac{\kappa \bar{x}_{*}}{(\sigma-f)}$ will result in a sublayer thickness approximately 0.39 times its former value and eddy viscosities that are significantly smaller than those obtained from previous model results.

Numerical results using $l=\frac{1}{6} \cdot 0.39 \frac{\kappa \bar{u}_{*}}{(\sigma-f)}$ are shown in Figures 34-37 and are presented according to previous results depicted in Figures 30-33, respectively. Pressure forcing and specified bottom roughness are the same as before. It can be seen from Figure 34 that magnitudes $R^{+}$and $R^{-}$as well as current amplitudes $U_{M a j}$ and $U_{M i n}$ agree much better with measured current speeds in the lower part of the water column. Furthermore, phase angles now display greater veering than in Figure 30f, and behave very much as suggested by the observations. The vertical structure of the inclination of the current ellipse is hardly changed, with the major axis rotating clockwise by $\approx 2^{\circ}$ between the surface and $30 \mathrm{~m}$ above the seafloor, and anticlockwise by $\approx 4^{\circ}$ in the rest of the water column. With $\bar{u}_{*}=1.25 \mathrm{~cm} / \mathrm{s}$ based on model results, the height of the region in which $K$ increases linearly away from the bottom is $l \approx 7.0 \mathrm{~m}$. According to Figure 35 , the vertical average of numerical eddy viscosities at heights less than $25 \mathrm{~m}$ can be computed to $300 \mathrm{~cm}^{2} / \mathrm{s}$, a value much closer to observed magnitudes than previous model results. Deviations from the best-fit logarithmic profile to numerical current speeds between the seafloor and $2.75 \mathrm{~m}$ above the bottom are less than $1 \%$ for $z<7.25 \mathrm{~m}$, and approach $10 \%$ near the surface. Stress ellipse parameters obtained from numerical data display good agreement with those computed from measured velocities in the vicinity of the sea floor, with predicted bottom stress magnitudes being within $10 \%$ of those derived from BASS data (Figure 36). In addition, there is more evidence of a region of small stress magnitudes at heights $>40 \mathrm{~m}$ than it was the case in the first modeling study. Velocity 
defects computed from $\int_{0}^{z}\left(\mathbf{R}^{ \pm}-\mathbf{R}_{s}^{ \pm}\right) d z$ are shown in Figure 37 . It can be seen from the figure that predicted velocity defects are closer to their measured values than in Figure 33, confirming that it is the under-estimation of current speeds in the lower part of the water column which accounts for the largest part of observed discrepancies with previous model results.

Despite major improvements of predicted current amplitudes, bottom stresses, and velocity defects using $l=\frac{1}{6} \cdot 0.39 \frac{\kappa \bar{u}_{*}}{\sigma-f}$, estimates for the logarithmic-layer thickness as well as magnitudes of numerical eddy viscosities are still large compared to values infered from the observations. Numerical investigation shows that model results can be further improved if the sublayer height is reduced to $l=\frac{1}{10} \cdot 0.39 \frac{\kappa \bar{u}_{*}}{\sigma-f}$. With $\delta=0.39 \frac{\kappa \bar{u}_{*}}{\sigma-f}$ being the approximate location where the transition to free-stream conditions occurs, the above parameterization yields $l=0.1 \delta$ and is representative of the scaling height known to describe the logarithmic-layer-thickness for steady and rectilinear flows. This is in agreement with results from measured data discussed in III.2.6., suggesting the observed thickness of the logarithmic layer to be of order $4 \mathrm{~m}$, and thus approximately one-tenth the height of $\delta \approx \delta^{-} \approx 40 \mathrm{~m}$ derived from current amplitudes $R^{-}$.

Model results applying $l=\frac{1}{10} \cdot 0.39 \frac{\kappa \bar{u}_{*}}{\sigma-f}$ are shown together with data in Figures 38-41 for the current ellipse, vertical mixing coefficient, stress ellipse, and boundary layer transports, respectively. It can be seen from Figures 38a and e that current amplitudes in the lower part of the water column are predicted with even greater accuracy than in Figure 34. Similar statements apply to the phase angles $\Delta \phi^{+}, \Delta \phi^{-}$, and $\Delta \phi$, and the inclination of the major axis, $\Delta \theta$. With numerical friction velocities $\bar{u}_{*}=1.19 \mathrm{~cm} / \mathrm{s}$, the sublayer extends to $l=4 \mathrm{~m}$, a value very similar to the height of the logarithmic region observed in actual current profiles. Model results suggest deviations from best-fit logarithmic profiles to current speeds between 0.25 and $2.75 \mathrm{~m}$ above the bottom to be less than $1 \%$ at heights $z<5.75 \mathrm{~m}$, and to reach $5 \%$ and $16 \%$ at $z \approx 12 \mathrm{~m}$ and $z \approx 40 \mathrm{~m}$, respectively. Thus, the model still over-estimates the extent of the logarithmic region, but in contrast to earlier results, deviations from the logarithmic fit are significant in at least two-thirds of the water column. Depth- 
averaged numerical eddy viscosities between the bottom and $z=25 \mathrm{~m}$ are $174 \mathrm{~cm}^{2} / \mathrm{s}$, in close agreement with $162 \mathrm{~cm}^{2} / \mathrm{s}$ computed from the observations (Figure 39). Stress ellipse parameters obtained from numerical data are shown in Figure 40 and display good agreement with those computed from measured velocities. This is particularly true in the lowest $12 \mathrm{~m}$ of the water column, where similarities of their vertical structure are more obvious than in Figures 32 and 36. Numerical bottom stress estimates are within $1 \%$ of the stress magnitudes derived from BASS data. Predicted velocity defects $\int_{0}^{z}\left(\mathbf{R}^{ \pm}-\mathbf{R}_{s}^{ \pm}\right) d z$ reproduce results obtained from observations better than any of the previously discussed model runs (Figure 41).

Based on the above numerical model results, it can be concluded that from all parameterizations discussed in this section, the $K$-profile model with $l=\frac{1}{10}$. $0.39 \frac{\kappa \bar{u}_{*}}{(\sigma-f)}$ reproduces the observations most closely. With $\delta=0.39 \frac{\kappa \bar{u}_{*}}{(\sigma-f)}$ as a representative thickness of the total boundary layer, this behavior suggests that a meaningful scaling for the vertical extent of the sublayer is given by the height of the logarithmic layer shown to be of order $0.1 \delta$. Although observations indicate that the approximation $K=\kappa \bar{u}_{*} z$ is valid to a lesser degree than the assumption of a logarithmic flow region, specifying $l=0.1 \delta$ is necessary in order to account for the fact that actual eddy viscosities increase throughout the entire boundary layer (Figure 28), and to predict depth-averaged values for $K$ similar to those obtained from measured data. However, it is not only the close agreement in magnitudes of depth-averaged mixing coefficients that is responsible for the good performance of the $K$-profile model with $l=0.1 \delta$. Numerical investigation shows it to be essential that $K=\kappa \bar{u}_{*} z$ is applied in at least some limited region near the bottom, in accordance with the logarithmic law of the wall and the assumption of a constant stress layer. For example, using $K=\frac{1}{3} \kappa \bar{u}_{*} z$ in the entire boundary layer, in qualitative agreement with Figure 28b, leads to model results which under-estimate actual bottom stresses by more than $50 \%$, and velocity profiles that do not resemble measured data in the lower part of the water column. 


\section{III.3.2.2 Performance of the MY 2.5}

Model results using the MY 2.5 turbulence closure scheme are shown together with results from actual data in Figures $42-45$ for the vertical structure of the current ellipse, eddy viscosity, stress ellipse, and boundary layer transports, respectively. Solid lines in Figure 43 represent numerical mixing coefficients according to their lowest and highest values during one tidal cycle. For comparison, the dashed line depicts the slope $\kappa \bar{u}_{*} z$, where $\bar{u}_{*}=1.27 \mathrm{~cm} / \mathrm{s}$ is taken from model results. It can be seen from Figure 42 that profiles of current ellipse parameters resemble closely those displayed in Figure 30. Consequently, deficiencies with respect to actual data are similar to the $K$-profile model using $l=\frac{1}{6} \cdot \frac{\kappa \bar{u}_{*}}{\sigma-f}$, with current speeds being over-estimated at heights less than $\approx 25 \mathrm{~m}$ above the bottom (Figures $42 \mathrm{a}$ and $\mathrm{d}$ ), and velocity defects $\int_{0}^{z}\left(\mathbf{R}^{-}-\mathbf{R}_{s}^{-}\right) d z$ that significantly under-predict the observed values (Figure 45 ). The reason for this behavior is that similar to previous results, eddy viscosities obtained from the MY 2.5 are noticeably larger than those computed from velocity measurements in the lower part of the water column (Figure 43). Their time-mean vertical average in the lowest $25 \mathrm{~m}$ of the water column is $353 \mathrm{~cm}^{2} / \mathrm{s}$, a value more than twice as large as $162 \mathrm{~cm}^{2} / \mathrm{s}$ based on observation. As a consequence, the MY 2.5 over-emphasizes the importance of vertical mixing in the lower part of the water column, resulting in model speeds that are too large in comparison with actual data (Figures 42a and d), and velocity defects that do not agree with those computed from measurements (Figure 45). Similar to results of the $K$-profile model for $l=\frac{1}{6} \cdot \frac{\kappa \bar{u}_{*}}{\sigma-f}$, deviations from the best-fit logarithmic profiles to numerical data between 0.25 and $2.75 \mathrm{~m}$ above the bottom are less than $6 \%$ over the entire water depth. However, they are smaller than $1 \%$ only in the lowest $5.25 \mathrm{~m}$ of the water column as opposed to $10 \mathrm{~m}$ for the two-layer model quoted above. This behavior might explain why, despite deficiencies in the prediction of current amplitudes and boundary layer transports, MY 2.5 does perform reasonably well in the estimation of bottom stress magnitudes (Figure 44). Predicted bottom stress estimates are within $12 \%$ of the magnitudes derived from BASS data.

In conclusion, it can be said that the MY 2.5 predicts the observed bottom stresses with considearble accuracy, but does not compare significantly better with observations 
of current ellipse parameters or velocity defects than the $K$-Profile model using $l=\frac{1}{6}$. $\frac{\kappa \bar{u}_{*}}{\sigma-f}$. Model results applying the $K$-profile closure with $l=0.1 \delta$, where $\delta=0.39 \frac{k \bar{u}_{*}}{(\sigma-f)}$ according to the observed height at which free-stream conditions are reached, show better agreement with actual data than any other numerical model discussed in this study. 


\section{Vertical Structure of the Subtidal Flow}

\section{IV.1 Introduction}

In this chapter, the bottom boundary layer structure of the subtidal flow will be investigated. The term subtidal refers to frequencies less than diurnal, with higher frequencies being excluded by the PL64 (Beardsley et al., 1983) low-pass-filter. Generation mechanisms for the subtidal flow on the southern flank of Georges Bank are the mean density field (Flagg et al., 1982), tidal rectification (Huthnance, 1973; Zimmermann, 1980, Loder, 1980), and wind forcing (Brink et al., 1987). In addition, water is provided to the southern flank by the Labrador Current moving southward along the continental slope after passing the Grand Banks of Newfoundland. Mean density gradients at ST1 are mostly across-bank and associated with the tidal mixing front to the northwest, and the shelf slope front to the southeast. During winter, the tidal mixing front is not present, and horizontal stratification is set by the gradual transition from fresh cool shelf water to salty warm slope water in the offbank direction. Salinity outweighs the effects of temperature, resulting in a slight off-bank increase of density. CTD cross-sections taken in February and March 1995 reveal density gradients to be of order $\Delta \sigma_{t} \approx 0.1$ over a $20-\mathrm{km}$ distance centered at ST1, resulting in a thermal wind of $\frac{\Delta u}{\Delta z} \approx 5 \cdot 10^{-4} \mathrm{~s}^{-1}$. With the water depth at ST1 being $76 \mathrm{~m}$ and assuming no flow at the bottom, the associated depth-averaged flow speeds are of order $2 \mathrm{~cm} / \mathrm{s}$ and clockwise around the bank. Tidal rectification similarly leads to a clockwise mean current, thus adding to the density-driven flow. The magnitude of the rectified current is not well known, but Loder's (1980) idealized depth independent model suggests mean flow

speeds to be of order $2 \mathrm{~cm} / \mathrm{s}$ along the $76 \mathrm{~m}$ isobath. According to numerical results by Chen (1992), subtidal along-bank velocities are around $2.4 \mathrm{~cm} / \mathrm{s}$ near the surface, if the water column is unstratified. For weakly stratified flow $\left(N^{2} \approx 10^{-5} \mathrm{~s}^{-2}\right)$, Chen (1992) computes surface velocities of $\approx 5 \mathrm{~cm} / \mathrm{s}$ at the same water depth, largely associated with the horizontal density gradient in the bottom mixed layer over the flank of the 
bank. Since no major wind events occurred between yearday 50 and 80 (Figure 5), the wind-driven flow is mostly confined to the upper part of the water column.

In the following investigation, it was attempted to exclude periods of significant mean flow caused by horizontal density gradients. This was done in order to avoid the effects of thermal wind currents on the vertical structure of the bottom boundary layer, since the presence of a well-defined interior flow with little vertical shear greatly simplifies the scaling of the boundary layer thickness. The study furthermore focuses on steady boundary layers, i.e., on times where the acceleration of the mean flow is weak.

\section{IV.2 Governing Equations}

Making use of the Boussinesq approximation $\rho \approx \rho_{0}$, but without applying further simplifications, the horizontal momentum equations governing the subtidal flow can be written as

$$
\begin{aligned}
& \frac{\partial \bar{u}}{\partial t}+\overline{u \frac{\partial u}{\partial x}}+\overline{v \frac{\partial u}{\partial y}}-f \bar{v}=-\frac{1}{\rho_{0}} \frac{\partial \bar{p}}{\partial x}+\frac{1}{\rho_{0}} \frac{\partial \bar{\tau}^{x}}{\partial z} \\
& \frac{\partial \bar{v}}{\partial t}+\overline{u \frac{\partial v}{\partial x}}+\overline{v \frac{\partial v}{\partial y}}+f \bar{u}=-\frac{1}{\rho_{0}} \frac{\partial \bar{p}}{\partial y}+\frac{1}{\rho_{0}} \frac{\partial \bar{\tau}^{y}}{\partial z}
\end{aligned}
$$

In (42), overbars denote the time average, and $x$ and $y$ are along- and off-bank directions, respectively. The along-bank direction was taken to be perpendicular to the orientation of the $\mathrm{M}_{2}$ tidal pressure forcing and is $-149^{\circ}$ from east. Unlike the case of the total flow given in (26), nonlinear advective terms cannot be neglected in the description of the subtidal currents. This is due to the fact that advective terms on the left-hand sides of (42) contain the contribution of tidal currents, while Coriolis terms describe the effects of rotation on the time averaged flow. The ratio of advective to Coriolis terms is given by $\frac{U^{2}}{\overline{U f L}}$, where $U$ and $\bar{U}$ scale as the magnitudes of the tidal and subtidal flow, respectively. With $U \approx 40 \mathrm{~cm} / \mathrm{s}$ and $L \approx 40 \mathrm{~km}$ used in III.2.2, and with $\bar{U} \approx 10 \mathrm{~cm} / \mathrm{s}$ according to Figure $2, \frac{U^{2}}{\overline{U f L}} \approx \kappa$. Thus, advective 
terms are significant and cannot be omitted a priori in agreement with the presence of a tidally rectified current as described by Huthnance (1973). This is particularly true in (42a), since subtidal velocities on the southern flank of Georges Bank are clockwise and around-bank in the absence of strong wind forcing or advective processes associated with motion of the shelf-slope front. Consequently, $\bar{v}$ is generally much less than $\bar{u}$, and along-bank pressure gradients are generally small compared to subtidal pressure forcing in the cross-bank direction.

Under the assumption of steady flow, vertical integration of (42) yields

$$
\begin{gathered}
-\int_{0}^{D} f \bar{v} d z=-\int_{0}^{D}\left(\frac{1}{\rho_{0}} \frac{\partial \bar{p}}{\partial x}+\overline{u \frac{\partial u}{\partial x}}+\overline{v \frac{\partial u}{\partial y}}\right) d z+\frac{1}{\rho_{0}} \bar{\tau}_{w}^{x}-\frac{1}{\rho_{0}} \bar{\tau}_{b}^{x} \\
\int_{0}^{D} f \bar{u} d z=-\int_{0}^{D}\left(\frac{1}{\rho_{0}} \frac{\partial \bar{p}}{\partial y}+\overline{u \frac{\partial u}{\partial x}}+\overline{v \frac{\partial u}{\partial y}}\right) d z+\frac{1}{\rho_{0}} \bar{\tau}_{w}^{x}-\frac{1}{\rho_{0}} \bar{\tau}_{b}^{x}
\end{gathered}
$$

Defining the depth integral of the total pressure gradient to be

$$
\begin{aligned}
& \frac{1}{\rho_{0}} \int_{0}^{D} \frac{\partial \bar{p}^{T}}{\partial x} d z=\int_{0}^{D}\left(\frac{1}{\rho_{0}} \frac{\partial \bar{p}}{\partial x}+\overline{u \frac{\partial u}{\partial x}}+\overline{v \frac{\partial u}{\partial y}}\right) d z, \\
& \frac{1}{\rho_{0}} \int_{0}^{D} \frac{\partial \bar{p}^{T}}{\partial y} d z=\int_{0}^{D}\left(\frac{1}{\rho_{0}} \frac{\partial \bar{p}}{\partial y}+\overline{u \frac{\partial v}{\partial x}}+\overline{v \frac{\partial v}{\partial y}}\right) d z,
\end{aligned}
$$

$\frac{\partial \bar{p}^{T}}{\partial x}$ and $\frac{\partial \bar{p}^{T}}{\partial y}$ contain the barotropic and baroclinic contributions to horizontal pressure variations as well as the nonlinear advective terms. Equations $43 \mathrm{a}, \mathrm{b}$ can then be written as

$$
\begin{gathered}
-\int_{0}^{D} f \bar{v} d z=-\frac{1}{\rho_{0}} \int_{0}^{D} \frac{\partial \bar{p}^{T}}{\partial x} d z+\frac{1}{\rho_{0}} \bar{\tau}_{w}^{x}-\frac{1}{\rho_{0}} \bar{\tau}_{b}^{x}, \\
\int_{0}^{D} f \bar{u} d z=-\frac{1}{\rho_{0}} \int_{0}^{D} \frac{\partial \bar{p}^{T}}{\partial y} d z+\frac{1}{\rho_{0}} \bar{\tau}_{w}^{y}-\frac{1}{\rho_{0}} \bar{\tau}_{b}^{y},
\end{gathered}
$$


This representation was chosen since the magnitude and vertical structure of the advective terms are unknown, rendering the application of (43) to data analysis difficult. On the other hand, it is possible to compute $\frac{1}{\rho_{0}} \int_{0}^{D}\left(\frac{\partial \bar{p}^{T}}{\partial x}, \frac{\partial \bar{p}^{T}}{\partial y}\right) d z$ from (45) based on low-pass-filtered flow speeds and surface stresses obtained from current meter and IMET data and bottom stress estimates derived from BASS data. Subtidal bottom stresses were obtained by low-pass-filtering

$$
\frac{1}{\rho_{0}}\left(\tau_{b}^{x}, \tau_{b}^{y}\right)=c_{D} \sqrt{u^{2}+v^{2}}(u, v),
$$

where $c_{D}$ is a drag coefficient, and $u$ and $v$ are the corresponding total along- and cross-bank velocities, respectively. Equation 46 was used, since it is believed that the periodical variation of bottom roughness estimates associated with the battery case significantly affects the low-passed version of bottom stress values derived from best-fit logarithmic profiles. The quadratic drag law was applied to pods 1-3, and results were averaged to obtain a time series of bottom stress estimates. Drag coefficients at the respective heights were obtained from least-squares fits of $U^{2}$ to $u_{*}^{2}$.

\section{IV.3 Data Processing}

Since the bottom boundary layer structure of the subtidal flow is to be examined under steady, barotropic conditions, events of large acceleration $\frac{\partial \bar{u}}{\partial t}$ and $\frac{\partial \bar{v}}{\partial t}$ as well as times where horizontal density gradients were significant need to be excluded from the analysis. In order to find such events, subtidal velocities, surface, and bottom stresses were averaged in 12.42-hour (one $M_{2}$ period) bins, according to one $\mathrm{M}_{2}$ period. For each bin, time mean ratios $\frac{\partial \bar{u}}{\partial t} / f \bar{v}$ and $\frac{\partial \bar{v}}{\partial t} / f \bar{u}$ were computed. Events where the magnitude of either expression exceeded 0.1 at any height between the seafloor and $40 \mathrm{~m}$ above the bottom were excluded from the analysis. Next, the baroclinic pressure gradient in cross-bank direction was estimated according to

$$
\frac{\partial \bar{p}^{b c}}{\partial y}=g \frac{\partial \bar{\rho}}{\partial y}(D-z) \approx g \frac{\Delta \sigma_{\theta}}{l_{\mathrm{M}_{2}}}(D-z),
$$


where $\Delta \sigma_{\theta}$ is the variation of $\sigma_{\theta}$ during one tidal cycle, and $l_{\mathrm{M}_{2}}$ is the tidal excursion length scale of the $\mathrm{M}_{2}$ component. Taking $\Delta \sigma_{\theta}$ from Seacat data (Figure 6), and $l_{\mathrm{M}_{2}}=\frac{2 V}{\sigma}$ where $V$ is the amplitude of the cross-bank tidal flow, and $\sigma$ is the frequency of the $M_{2}$ tide, the baroclinic pressure gradient can be computed as a function of $z$. A measure for the relative importance of baroclinicity is given by

$$
r_{b c}=\frac{\int_{0}^{D} \frac{\partial \bar{p}^{b c} d z}{\partial y}, \int_{0}^{D} \frac{\partial \bar{p} T}{\partial y} d z}{,}
$$

which relates the depth integrated total pressure gradient, defined in (44), to the baroclinic contribution due to cross-bank density gradients. In order for effects of horizontal stratification to be negligible, it was specified that magnitudes of $r_{b c}$ obtained from bin-averaged values for $\frac{\partial \vec{p}^{b c}}{\partial y}$ and $\frac{\partial \bar{p}^{T}}{\partial y}$ should not exceed 0.1. In addition, it was required that $\left|\int_{0}^{D} \frac{\partial \bar{p}^{T}}{\partial x} d z / \int_{0}^{D} \frac{\partial \bar{p}^{T}}{\partial y} d z\right|<0.2$ in order to ensure that advective processes associated with significant cross-bank flow leading to enhanced magnitudes of the nonlinear terms on the left-hand sides of (42) are excluded from the analysis. In most cases, such events coincide with times of large $r_{b c}$ ratios, since they occur together with significant horizontal stratification related to the on-bank motion of the shelf slope front.

\section{IV.4 Interior Flow Regime}

Nine distinct events representing time averages of 12.42 hours each met the requirements of negligible acceleration and thermal wind. All events have in common that an interior flow regime exists between the bottom and the surface mixed layers at heights 12 to $45 \mathrm{~m}$ above the bottom. In this region, vertical shears are small, suggesting that the interior flow is to lowest order barotropic. Defining

$$
U_{0}=-\frac{1}{\rho_{0} f D} \int_{0}^{D} \frac{\partial \bar{p}^{T}}{\partial y} d z
$$




$$
V_{0}=\frac{1}{\rho_{0} f D} \int_{0}^{D} \frac{\partial \bar{p}^{T}}{\partial x} d z
$$

$U_{0}$ and $v_{0}$ represent subtidal velocities that might be expected in the free stream, if vertical variations of $\frac{\partial \bar{p}^{T}}{\partial x}$ and $\frac{\partial \bar{p}}{\partial y}$ were small. With times of significant horizontal stratification being excluded from the analysis, validity of (49) in the free stream requires that advective terms in (44) are either small computed to the barotropic pressure forcing, or that they are only weak functions of $z$. The latter is not to be expected, since magnitudes of advective terms are largely determined by the phase veering of the $\mathrm{M}_{2}$ velocities with respect to their free stream values, and by increasing tidal amplitudes as the surface is approached. Taking $U_{\infty}$ and $V_{\infty}$ as characteristic interior velocities derived from subtidal current profiles for each of the nine events mentioned above, and rotating the coordinate system such that the $\mathrm{x}$-axis is aligned with the vector $\left(U_{0}, V_{0}\right)$ computed from (49), corresponding velocities $\tilde{U}_{\infty}, \tilde{V}_{\infty}$, and $\tilde{U}_{0}$ are obtained. In the new coordinate system, $\tilde{V}_{0}=0$ by definition. A measure for the extent to which (49) applies is given by $\frac{\tilde{U}_{\infty}}{\tilde{U}_{0}}$, and $\frac{\tilde{V}_{\infty}}{\tilde{U}_{0}}$. The closer these ratios are to 1 and 0 , respectively, the better is the agreement of interior flow velocities with (49). Figure 46 shows the results of this calculation, with dotted lines representing the outer margins of the corresponding error bars. Uncertainties were obtained from linear error propagation. Since uncertainties of estimated bottom stresses associated with the least-squares logarithmic fit are not known for the subtidal frequency band, it was assumed that they cannot be larger than those compared for the total stress vector. It can be seen from the figure that measured velocities $\left(\tilde{U}_{\infty}, \tilde{V}_{\infty}\right)$ are within an arc of $\approx 24^{\circ}$ width from $\left(\tilde{U}_{0}, 0\right)$, with their magnitudes being similar to $\tilde{U}_{0}$. It can therefore be concluded that (49) represents the interior flow reasonably well, if acceleration of the subtidal currents and horizontal density gradients are weak. For all nine events depicted in Figure 46, the interior flow is roughly along-isobath at angles $\approx-149^{\circ}$ from east. The reason for this behavior is that significant cross-bank flow is either wind-driven, or due to advection at the shelf slope front. Both processes are 
likely to violate the requirement of quasi-steady flow. In addition, major advective events usually coincide with times of large horizontal stratification.

\section{IV.5 Scaling of the Subtidal Boundary Layer Height}

Velocity profiles of the subtidal flow representative of times where effects of acceleration and horizontal density gradients are small are shown in Figure 47. Velocities depicted in Figures $47 \mathrm{a}$ and $\mathrm{b}$ are averaged data, where the average was taken of all nine events meeting the requirements of quasi-steadiness and small baroclinic pressure forcing. Figures $47 \mathrm{c}$ and $\mathrm{d}$ display standard variations from the mean. It can be seen from Figures $47 \mathrm{a}$ and $\mathrm{b}$ that the vertical structure of subtidal currents in the lower part of the water column greatly resembles an Ekman spiral, with the interior flow being along-isobath. In the 20-30 m nearest to the surface, the flow is mainly wind-driven, and strong veering of the subtidal flow vector occurs. At approximately $12 \mathrm{~m}$ above the bottom, the mean value $\bar{u}$ approaches an interior value of $12-13 \mathrm{~cm} / \mathrm{s}$, and the mean of the cross-bank component $\bar{v}$ indicates that a half-turn of the bottom Ekman spiral has been completed. Defining

$$
\delta^{S T}=c \frac{\kappa \bar{u}_{*}}{f}
$$

where $\delta^{S T} \approx 12 \mathrm{~m}$ is the bottom boundary layer height based on subtidal current speeds from BASS and VMCM data, and taking $\bar{u}_{*} \approx 1.2 \mathrm{~cm} / \mathrm{s}$ from best-fit logarithmic profiles discussed in II.4.1, the constant $c \approx 0.24$. This is in reasonable agreement with results derived in II.2.3 suggesting $c \approx 0.39$ for $\delta^{ \pm}=c \frac{\kappa \bar{u}_{*}}{\sigma \pm f}$ according to $\mathrm{M}_{2}$ current amplitudes. It might therefore be suspected that the approximations

$$
\begin{aligned}
& \frac{\delta^{S T}}{\delta^{+}} \approx \frac{\sigma+f}{f}, \\
& \frac{\delta^{S T}}{\delta^{-}} \approx \frac{\sigma-f}{f},
\end{aligned}
$$


are valid to some limited degree. With $\delta^{S T} \approx 12 \mathrm{~m}, \delta^{+} \approx 8 \mathrm{~m}$, and $\delta^{-} \approx 40 \mathrm{~m}$, the left-hand sides of (51) are within $40 \%$ of their corresponding values on the righthand sides. This result indicates that the boundary layer thicknesses for tidal and subtidal flows are subject to similar parameterization, if the appropriate time scales $\frac{1}{f}, \frac{1}{(\sigma+f)}$, and $\frac{1}{(\sigma-f)}$ are used for the scaling of $\delta^{S T}, \delta^{+}$, and $\delta^{-}$, respectively. Since the subtidal flow is not influenced by temporal variations at tidal frequencies, bottom friction is balanced by the Coriolis force, and to some unknown extent by the nonlinear advective terms. A meaningful scale height over which effects of rotation oppose those of friction is presented in (50). Effects of tidal currents on the vertical structure of the subtidal flow enter (50) through the time mean friction velocity $\bar{u}_{*}$, which to lowest order is set by the amplitude of the $\mathrm{M}_{2}$ velocities. It is impossible to decouple the subtidal from the tidal flow, a statement that applies to the time-mean momentum equations (42) as well as to the scaling of the low-frequency boundary layer thickness given in (50).

\section{IV.6 Subtidal Boundary Layer Transports}

It was shown in IV.4 that the geostrophic velocities $U_{0}$ and $V_{0}$ defined in (49) represent the interior flow well, if acceleration of the subtidal currents and horizontal density gradients are weak. Based on this result, it shall now be examined whether subtidal velocity defects can to lowest order be expressed as

$$
\begin{aligned}
& \bar{T}^{x}=\int_{0}^{z}\left(\bar{u}-U_{0}\right) d z \\
& \bar{T}^{y}=\int_{0}^{z}\left(\bar{v}-V_{0}\right) d z
\end{aligned}
$$

where $U_{0}$ is along-bank and $-149^{\circ}$ from east, and $V_{0}$ is across-bank. If nonlinear advection terms are negligible, or if they are only weak functions of $z,(49)$ almost exactly describes the free stream. In that case, vertical integration of (42) yields for 
steady flow

$$
\begin{gathered}
\int_{0}^{z}\left(\bar{v}-V_{0}\right) d z=-\frac{1}{\rho_{0} f}\left[\bar{\tau}^{x}(z)-\bar{\tau}_{b}^{x}\right] \\
\int_{0}^{z}\left(\bar{u}-U_{0}\right) d z=\frac{1}{\rho_{0} f}\left[\bar{\tau}^{y}(z)-\bar{\tau}_{b}^{y}\right] .
\end{gathered}
$$

Assuming that in the flow interior $\bar{\tau}^{x}=\bar{\tau}^{y}=0,(53)$ reduces to

$$
\begin{gathered}
\int_{0}^{\delta^{S T}}\left(\bar{v}-V_{0}\right) d z=\frac{1}{\rho_{0} f} \bar{\tau}_{b}^{x}, \\
\int_{0}^{\delta^{S T}}\left(\bar{u}-U_{0}\right) d z=-\frac{1}{\rho_{0} f} \bar{\tau}_{b}^{y},
\end{gathered}
$$

where $\delta^{S T}$ is the height at which subtidal free-stream conditions are reached. In order to investigate the validity of (54), velocity defects were computed from (52), and results were compared to the right-hand sides of (54). If (54) applies to some degree, profiles $\bar{T}^{x}(z)$ and $\bar{T}^{y}(z)$ must be similar to values $\frac{\bar{\tau}_{b}^{x}}{\rho_{0} f}$ and $-\frac{\bar{\tau}_{b}^{y}}{\rho_{0} f}$ in some limited region in the interior flow regime.

Figure 48 displays the integrated velocity defects according to (52) (solid lines) in comparison to values from the left-hand sides of (54) (dashed lines). Results represent the average of the velocity defects and boundary layer transport computed for each of the nine events when acceleration and horizontal stratification were small. Dotted lines depict the standard deviation from the mean. It can be seen from Figure 48a that subtidal velocity defects $\bar{T}^{x}=\int_{0}^{z}\left(\bar{u}-U_{0}\right) d z$ intersect the line $-\frac{\bar{\tau}_{b}^{y}}{\rho_{0} f}$ at $z \approx 12 \mathrm{~m}$, and remain almost constant for $12 \mathrm{~m}<z<45 \mathrm{~m}$. This is in good agreement with earlier results indicating the height of the subtidal boundary layer to be $\delta^{S T} \approx 12 \mathrm{~m}$, and the interior flow regime to extend to $\approx 45 \mathrm{~m}$ above the bottom. Figure 48a presents evidence that (54b) is valid to lowest order, thus suggesting that the nonlinear terms $\overline{u \frac{\partial v}{\partial x}}$ and $\overline{v \frac{\partial v}{\partial y}}$ are either small or only weak functions of $z$. As mentioned before, the latter is not likely to be true, leaving the possibility that advected terms might not be significant in the across-bank momentum equation. An explanation for 
this behavior can be found if it is assumed that $\frac{\partial(u, v)}{\partial x}<<\frac{\partial(u, v)}{\partial y}$ in accordance with characteristic along- and cross-bank length scales and with $u=O(v)$. Making the rigid-lid approximation, the continuity equation can then be expressed as

$$
D \frac{\partial v}{\partial y}+v \frac{\partial D}{\partial y}=0
$$

and leads to the scaling argument

$$
\frac{\Delta v}{\Delta y} \approx \frac{v}{D} \frac{\Delta D}{\Delta y}
$$

With typical values $v \approx 40 \mathrm{~cm} / \mathrm{s}$ and $\frac{\Delta D}{\Delta y} \approx 8 \cdot 10^{-4}$ at ST1 and $\frac{\Delta v}{\Delta y} \approx 4$. $10^{-6} \frac{1}{s}$. Comparing the magnitudes of the advective and Coriolis term in the cross-bank momentum equation yields $v \frac{\Delta v}{\Delta y} / f \bar{u} \approx 0.14$, where $\bar{u}$ was taken as $\approx 12 \mathrm{~cm} / \mathrm{s}$. On the other hand, Figure 48b reveals a large imbalance between $\bar{T}^{y}=\int_{0}^{z}\left(\bar{v}-V_{0}\right) d z$ and $\frac{\bar{\tau}_{b}^{x}}{\rho_{0} f}$, with integrated velocity defects being less than half the magnitude of the expected boundary layer transports everywhere in the water column. This behavior suggests that the nonlinear terms $\overline{u \frac{\partial u}{\partial x}}+\overline{v \frac{\partial u}{\partial y}}$ are significant, particularly since cross-bank velocities $\bar{v}$ are small. Neglecting their vertical structure in the computation of interior velocities as suggested by (49b) leads to an under-estimation of the actual integrated bottom boundary layer transports in the cross-bank direction. In order to exactly determine the influence of the advective terms on the velocity defect, their magnitudes and vertical structure need to be known.

In summary, the investigation of subtidal boundary layer transports supports the scaling of the boundary layer height $\delta^{S T}$ discussed in the preceding paragraph. It is also shown that advective terms play only a minor role in the time-averaged acrossbank momentum equation (42b), while their contribution to the time-mean along-bank momentum balance $(42 \mathrm{a})$ is significant. 


\section{Summary and Conclusions}

The vertical structure of the bottom boundary layer at ST1 $\left(40^{\circ} 51^{\prime} \mathrm{N}, 67^{\circ} 33^{\prime} \mathrm{W}\right)$ on the southern flank of Georges Bank was investigated for the time period from March 19, 1995 to February 20, 1995. During this time, vertical stratification was weak $\left(\approx 10^{-5} \mathrm{~s}^{-2}\right)$, and the lowest $30 \mathrm{~m}$ of the water column were nearly homogeneous. Tidal analysis of acoustic current meter (BASS) and VMCM data reveals that the largest contribution to the total flow is from the $\mathrm{M}_{2}$ tide, which contains $\approx 88 \%$ of the kinetic energy and reaches current amplitudes of $\approx 40 \mathrm{~cm} / \mathrm{s}$ in the upper part of the water column. Free-stream values of measured velocities as well as results from the depth integrated momentum equations show the $M_{2}$ pressure forcing to be nearly rectilinear and across-bank.

Bottom stress estimates were obtained from best-fit logarithmic profiles to BASS data taken at $0.22-2.53 \mathrm{~m}$ above the seafloor. A representative value for the bottom roughness was found to be $z_{0} \approx 0.7 \mathrm{~mm}$, and a time-average characteristic friction velocity is $u_{*}=(1.2 \pm \kappa) \mathrm{cm} / \mathrm{s}$. About $66 \%$ of the bottom stress variance is caused by the $M_{2}$ currents, which represent the major mechanism for turbulence production at the lower boundary. Bottom stress values at the $\mathrm{M}_{2}$ frequency are in reasonable agreement with results from the downward integrated velocity defect assuming an interior flow regime with zero stress divergence at heights larger than $35 \mathrm{~m}$ (Figure 26).

Investigation of the $\mathrm{M}_{2}$ currents shows that the clockwise rotating component, $\mathbf{R}^{-}$, is about five times the magnitude of the anticlockwise rotating component, $\mathbf{R}^{+}$(Figure 20a). Boundary layer heights $\delta^{-}$and $\delta^{+}$are $\approx 40 \mathrm{~m}$ and $8 \mathrm{~m}$ based on current amplitudes $R^{-}$and $R^{+}$, and $\approx 50 \mathrm{~m}$ and $10 \mathrm{~m}$ based on veering angles $\Delta \phi^{-}$and $\Delta \phi^{+}$(Figure 20b,c), respectively. The ratio $\frac{\delta^{-}}{\delta^{+}} \approx 5$ is consistent with the scaling argument $\delta^{ \pm}=c \frac{\kappa \bar{u}_{*}}{\sigma \pm f}$, which with $\sigma=1.41 \cdot 10^{-4} \frac{1}{\mathrm{~s}}$ and $f=0.95 \cdot 10^{-4} \frac{1}{\mathrm{~s}}$ yields $\frac{\delta^{-}}{\delta^{+}}=\frac{\sigma+f}{\sigma-f}$. This behavior is in partial agreement with earlier results from Soulsby (1990), suggesting $\left(\frac{\sigma+f}{\sigma-f}\right)^{1 / 2}<\frac{\delta^{-}}{\delta^{+}}<\frac{\sigma+f}{\sigma-f}$ for Celtic Sea data. Soulsby (1990) concluded that the observed ratio of tidal boundary layer thicknesses is associated with the vertical structure of the eddy viscosity. He found that profiles 
of vertical mixing coefficients are approximately linear in some limited region near the sea floor, and constant in the outer part of the boundary layer, thus leading to boundary layer heights which lie in between the corresponding scale heights $\delta^{ \pm} \approx \frac{\kappa \bar{u}_{*}}{\sigma \pm f}$ for $K=\kappa \bar{u}_{*} z$, and $\delta^{ \pm} \approx\left(\frac{K}{\sigma \pm f}\right)^{1 / 2}$ for $K=$ constant. In the present case, it is the first expression which best describes the observations, suggesting that a meaningful parameterization of $\delta^{+}$and $\delta^{-}$can be derived under the assumption that $K$ increases linearly with height. However, vertical mixing coefficients computed from measured velocity shears indicate that $K=\kappa \bar{u}_{*} z$ is valid only in the lowest $1.5-2.0 \mathrm{~m}$ of the water column, while eddy viscosities increase at about one-third that rate in the rest of the boundary layer (Figure 28). Observed values $\delta^{ \pm} \approx(0.39-\kappa 8) \frac{\kappa \bar{u}_{*}}{\sigma \pm f}$ therefore suggest that tidal boundary layer heights are determined by the magnitude of turbulent fluctuations in the immediate vicinity of the seafloor, leading to scaling arguments which are based on the logarithmic law of the wall. The vertical extent of the logarithmic layer is of order $4 \mathrm{~m}$, a value approximately one tenth of the total boundary layer thickness $\delta^{-} \approx 40-50 \mathrm{~m}$ (Figure 29). This result is in agreement with earlier studies on rectilinear and steady flows (Clauser, 1956; Grant et al., 1986; Grant, 1977; Jonsson et al., 1976), suggesting that the logarithmic region of such flows covers about $10 \%$ of the entire boundary layer.

Numerical results from a one-dimensional two-layer eddy viscosity model using the parameterization $K=\kappa \bar{u}_{*} z$ at heights $z \leq l$, and $K=\kappa \bar{u}_{*} l$ for $z>l$ indicate that measured current amplitudes, ellipse inclinations, and phase veering, as well as boundary layer transports and bottom stresses, can be reproduced closely if the sublayer thickness is taken as $l=0.1 \delta$, where $\delta$ is the total boundary layer height. Since the $M_{2}$ tide is dominated by the clockwise rotating component, the boundary layer thickness is given by $\delta \approx \delta^{-}$and can be parameterized according to $\delta=c \frac{\kappa \bar{u}_{*}}{\sigma-f}$, with $c \approx 0.39$ based on the observed current amplitudes $R^{+}$and $R^{-}$. Model results applying this parameterization are in good agreement with current ellipse parameters and boundary layer transports computed from measurements (Figures 38 and 41). Predicted bottom stresses are within $1 \%$ of estimates obtained from BASS data, if the numerical bottom roughness is taken as $z_{0} \approx 0.7 \mathrm{~mm}$ according to the value suggested by the observations (Figure 40 ). With $\bar{u}_{*}=1.19 \mathrm{~cm} / \mathrm{s}$ from model 
data, numerical friction velocities are almost identical to those computed from best-fit logarithmic profiles to measured current speeds. The sublayer height is $l=4 \mathrm{~m}$, a value representative of the logarithmic layer thickness deduced from measured velocity profiles. Closer investigation reveals that model results are sensitive to the specification of $l$, especially in the lower part of the boundary layer. For example, using $l=\frac{1}{6} \frac{\kappa \bar{u}_{*}}{\sigma-f}$ in agreement with the scaling $l=\frac{1}{6} \frac{\kappa \bar{u}_{*}}{\omega}$ for rectlinear flow introduced by Trowbridge et al. (1984) but taking the oscillation frequency $\omega=\sigma-f$ yields current amplitudes that over-estimate the measured speeds at heights smaller than $\approx 25 \mathrm{~m}$ above the bottom (Figure 30), and boundary layer transports that are too small in comparison with actual data (Figure 33). This behaviour is explained by the large values of numerical eddy viscosities, associated with the sublayer height extending to $l=20 \mathrm{~m}$ (Figure 31). Furthermore, numerical friction velocities exceed the estimates from best-fit logarithmic profiles by $\approx 13 \%$, leading to bottom stresses that are about 1.3 times the magnitude derived from BASS data (Figure 32). Results can be improved substantially, if $l=\frac{1}{6} \delta$ is applied, with $\delta=0.39 \frac{\kappa \bar{u}_{*}}{\sigma-f}$ being a realistic parameterization of the observed boundary layer thickness. However, measured velocities in the lower part of the water column are still slightly over-predicted (Figure 34), and numerical velocity defects do not match those computed from the observations very well (Figure 37).

Based on these results, it can be concluded that the performance of the eddy viscosity model is optimized, if the sublayer is scaled according to the observed thickness of the logarithmic layer. Extending the sublayer to greater heights implies that $K=$ $\kappa \bar{u}_{*} z$ is no longer a good approximation, since it is valid only in the limits of a logarithmic velocity profile and constant stress layer. Thus, taking $l>0.1 \delta$, where $\delta \approx \delta^{-}$is the observed height of the bottom boundary layer, results in numerical eddy viscosities which are too large in most of the water column, and overemphasizes the relative importance of turbulent mixing.

It is not clear at this point how the logarithmic layer thickness can best be parameterized if its vertical extent is not known. Observations suggest that the logarithmic layer covers about $10 \%$ of the total boundary layer height $\delta \approx \delta^{-} \approx c \frac{\kappa \bar{u}_{*}}{\sigma-f}$, so that 
an appropriate scaling can be derived if estimates of $c$ are available. Alternative parameterizations may be given by $\delta_{\sigma}=\frac{\kappa \bar{u}_{*}}{\sigma}$ and $\delta_{f}=\frac{\kappa \bar{u}_{*}}{f}$. With $c \approx 0.39$ deduced from current measurements, and $\bar{u}_{*}=1.2 \mathrm{~cm} / \mathrm{s}$ derived from BASS data, the boundary layer height $\delta=c \frac{\kappa \bar{u}_{*}}{\sigma-f}, \approx 40 \mathrm{~m}$ is within $18 \%$ and $20 \%$ of the scale heights $\delta_{\sigma} \approx 34 \mathrm{~m}$ and $\delta_{f} \approx 50 \mathrm{~m}$, respectively. According to earlier studies, $\delta_{\sigma}$ is a representative value for the boundary layer thickness of rectilinear flows (Trowbridge et al., 1984; Grant, 1977; Grant, 1986) if the oscillation is at the $\mathrm{M}_{2}$ frequency, and $\delta_{f}$ describes the approximate height of the planetary boundary layer. Taking $l=0.1 \delta_{\sigma}$ or $l=0.1 \delta_{f}$ yields the sublayer heights $3.4 \mathrm{~m}$ and $5.0 \mathrm{~m}$, respectively. Both values are reasonably close to $l=0.1 \delta=4.0 \mathrm{~m}$, and in good agreement with the observed logarithmic layer thickness. Numerical investigation shows that results using $l=0.1 \delta, l=0.1 \delta_{\sigma}$, and $l=0.1 \delta_{f}$ are very similar, making it impossible to conclude which of the above expressions is more appropriate. Thus, the fundamental question of how to parameterize the logarithmic layer thickness without prior knowledge of its vertical extent cannot be answered with certainty. An argument in favor of $l=0.1 \delta$ is that similar scaling was found to be true for rectilinear and steady flows quoted earlier, if $\delta$ is taken as the approximate height where free-stream conditions are reached. If, on the other hand, it is considered that the structure of the logarithmic layer is determined by current speeds, and does to lowest order remain unaffected by the rotation of the velocity vector, the physical picture is that of a mean current superimposed by an oscillation at the $\mathrm{M}_{2}$ frequency. This problem has been solved analytically in the limit of high-frequency oscillations $\omega$ (Grant et al., 1979), suggesting a wave boundary layer of height $\delta_{\omega}=\frac{\kappa \bar{u}_{*}}{\omega}$ to build up above the seafloor, which is then merged with the much larger planetary boundary layer of order $\delta_{f}=\frac{\kappa \bar{u}_{*}}{f}$. The vertical extent of the logarithmic layer might then be expected to scale according to $0.1 \delta_{f}$, with the sole influence of the waves lying in their contribution to the friction velocity $u_{*}$, and the increase of physical bottom roughness felt by the mean flow. In the present case, the frequency of the tidal oscillation is comparable to the value of $f$, so that $\delta_{f} \approx 50 \mathrm{~m}$, and $\delta_{\sigma} \approx 34 \mathrm{~m}$ are of the same order. It might therefore be concluded that the vertical extent of the logarithmic layer is limited by either of the two scale heights and is given by $\approx 0.1 \delta_{f}$ or $\approx 0.1 \delta_{\sigma}$. 
Comparison of the observations to model results using the Mellor-Yamada level 2.5 (MY 2.5) turbulence closure scheme shows that the numerical solutions display deficiencies similar to those observed for the two-layer model with $l>0.1 \delta$. Although bottom stresses and friction velocities are predicted with considerable accuracy (Figure 44), model velocities in the lower part of the water column are too large (Figure 42), and velocity defects are small compared to results obtained from actual data (Figure 45). Analogous to results from the two-layer model using sublayer heights larger than the observed thickness of the logarithmic layer, numerical eddy coefficients exceed those computed from measured current shears in most of the boundary layer (Figure 43), and the effects of turbulent mixing are over-predicted. Overall, the performance of the MY 2.5 is less convincing than that of the much simpler eddy-viscosity model with $l=0.1 \delta$.

Investigation of the subtidal flow reveals mean velocities to be along-bank and to the southwest, if cross-bank density gradients and acceleration of the subtidal currents are small. During these times, an interior flow regime exists at heights $\approx 12$ to $45 \mathrm{~m}$ above the seafloor, surrounded by the bottom and surface turbulent layers (Figure 47). Along-bank currents in the free stream are of order $12-13 \mathrm{~cm} / \mathrm{s}$ (Figures 46 and $47 \mathrm{a}$ ). Bottom boundary layers associated with the subtidal flow extend to $\delta^{S T} \approx 12 \mathrm{~m}$, corresponding to the height were the interior flow regime is approached. This value supports earlier results for the $\mathrm{M}_{2}$ constituent, suggesting that approximations $\frac{\delta^{S T}}{\delta^{-}} \approx$ $\frac{\sigma-f}{f}$ and $\frac{\delta^{S T}}{\delta^{+}} \approx \frac{\sigma+f}{f}$ are valid to some limited degree.

According to $\delta^{ \pm} \approx c \frac{\kappa \bar{u}_{*}}{\sigma \pm f}$ and $\delta^{S T} \approx c \frac{\kappa \bar{u}_{*}}{f}$ based on current speeds, tidal and subtidal boundary layer thicknesses are subject to similar scaling, and are set by the magnitude of turbulent fluctuations near the sea floor together with the time scale on which temporal variations occur. Since the $M_{2}$ tide represents the major mechanism by which bottom stresses and friction velocities are generated, the vertical extent of the subtidal boundary layer is largely determined by the amplitude of the tidal currents.

Examination of subtidal boundary layer transports reveals that integrated velocity defects for along-bank flow are similar to the value $-\frac{\bar{\tau}_{b}^{y}}{\rho_{0} f}$ at heights where free-stream conditions are reached (Figure 48a). This result indicates that nonlin- 
ear advective terms in the time-averaged cross-bank momentum balance (42b) are not significant, in agreement with the fact that along-bank velocities are to lowest order barotropic in the interior flow regime. An explanation for this behavior may be found by taking into account that the bottom slope is small at the mooring location, and that spatial variations of total current speeds are likely to be negligible in the along-bank direction. Under these assumptions, scaling of the continuity and subtidal cross-bank momentum equations suggests advection to be negligible compared to the Coriolis term. However, this is not true for the along-bank momentum balance (43a), since the low-passed cross-bank flow is weak. Integrated velocity defects of subtidal cross-bank currents do not approach the value $\frac{\bar{\tau}_{b}^{x}}{\rho_{0} f}$ anywhere in the water column (Figure 48b), supporting the statement that advective terms containing the time-mean tidal contributions are significant. It is the relative importance of advective and friction terms that provides the mechanism for tidal rectification, leading to an along-bank mean flow very much as suggested by Huthnance (1973), Zimmermann (1980), Loder (1980).

Future work might concentrate on the meaningful parameterization of the logarithmic layer thickness for application in the numerical two-layer model described above. In order to be able to predict the logarithmic layer height with greater certainty, data may be analyzed from locations where the ratio $\frac{R^{-}}{R^{+}}$differs from the present value. In particular, $R^{+} \gg R^{-}$would force a tidal boundary layer of height $\delta \approx \delta^{+} \approx \frac{\kappa \bar{u}_{*}}{\sigma+f}$, a thickness significantly smaller than for the reverse case $R^{-}>R^{+}$. Measurements of near-bottom velocities might clarify whether the vertical extent of the logarithmic region is described by any of the parameterizations $0.1 \delta$, $0.1 \delta_{f}$, or $0.1 \delta_{\sigma}$ suggested above, and whether its specification as the sublayer height gives numerical solutions that resemble the observations.

In addition, it is intended to investigate the bottom boundary layer structure under vertically stratified conditions. BASS and VMCM measurements are available for the period from June 11, 1995 to August 23, 1995, when solar heating causes vertical stratification to increase, and horizontal density gradients associated with the shelf slope and tidal mixing fronts are significant. Comparison of the tidal and subtidal boundary layer heights for stably stratified and vertically homogeneous flow may reveal 
information about the inhibiting effects of vertical stratification on turbulent mixing. Depending on the relative importance of such effects, the simple two-layer model suggested for the homogeneous case may no longer be sufficient, and the parameterization of vertical mixing may need to be modified. 


\section{References}

Beardsley, R. C., R. Limeburner, and L. K. Rosenfeld, 1985. Introduction to the CODE-2 moored array and large-scale data report. $\mathrm{R}$. Limeburner, Editor, Woods Hole Oceanographic Institution Technical Report WHOI-85-35.

Beardsley, R. C., J. Candela, R. Limeburner, W. R. Geyer, S. J. Lentz, B. M. Castro, D. Cacchione, and N. Carneiro, 1995. The $\mathrm{M}_{2}$ tide on the Amazon shelf. Journal of Geophysical Research, 100, 2283-2319.

Bisagni, J. J., D. J. Gifford, C. M. Ruhsam, 1996. The spatial and temporal distribution of the Maine Coastal Current during 1982. Continental Shelf Research, 16, 1.

Blumberg, A. F., and G. L. Mellor, 1987. A description of a three-dimensional coastal ocean circulation model. Coastal and Estuarine Science, 4, 1-16.

Brevik, I., 1981. Oscillatory rough turbulent boundary layers, Journal of Waterway, Port, Coastal Ocean Division of the American Society of Civil Engineers, 107(WW3), 175-188.

Brink, K. H., B. A. Magnell, and M. A. Noble, 1987. Low-frequency current and bottom pressure variability. In: Georges Bank, R. H. Backus, editor, The MIT Press, pp. 140-146.

Butman, B., J. W. Loder, and R. C. Beardsley, 1987. The seasonal mean circulation: Observation and theory. In: Georges Bank, R. H. Backus, editor, The MIT Press, pp. 125-138.

Chapman, D. C. and R. C. Beardsley, 1989. On the origin of shelf water in the Middle Atlantic Bight. Journal of Physical Oceanography, 19, 384-390.

Chen, C., 1992. Variability of currents in Great South Channel and over Georges Bank: Observation and Modeling. Ph.D. Thesis, Joint Program in Oceanography/ Applied Ocean Science and Engineering, Woods Hole Oceanographic Institution, Woods Hole, Mass, Massachusetts Institute of Technology, Cambridge, Mass.

Clauser, F. H., 1956. The turbulent boundary layer. Advances in Applied Mechanics, $4,1-51$.

Davies, A. M., and J. E. Jones, 1990. Application of a three-dimensional turbulence energy model to the determination of tidal currents on the northwest European shelf. Journal of Geophysical Research, 95, 18,143-18,162. 
Fang, G. and T. Ichiye, 1983. On the vertical structure of tidal currents in a homogeneous sea. Geophysical Journal of the Royal Astronomy Society, 73, 65-82.

Flagg, C. N., 1987. Hydrographic structure and variability. In: Georges Bank, R. H. Backus, editor. The MIT Press, pp. 108-124.

Fjeldstad, J. E., 1929. Contribution to the dynamics of free progressive tidal waves. Sci. Res. Norweg. North Polar Exped. "Maud" 1918-1925, Vol.4 (3). Geofysiks Institut, Bergen, pp. 1-80.

Grant, W. D, 1977. Bottom friction under waves in the presence of a weak current: Its relationship to coastal sediment transport. Sc.D. Thesis, Massachusetts Institute of Technology, Cambridge, Mass.

Grant, W. D., and O. S. Madsen, 1979. Combined wave and current interaction with a rough bottom. Journal of Geophysical Research, 84 (C4), 1797-1808.

Grant, W. D., and O. S. Madsen, 1986. The continental-shelf bottom boundary layer. Annual Reviews of Fluid Mechanics, 18, 265-305.

Huthnance, J. M., 1973. Tidal current asymmetries over the Norfolk Sandbank. Estuarine and Coastal Marine Science, 1, 89-99.

Jonsson, I. G., and N. A. Carlsen, 1976. Experimental and theoretical investigations in an oscillatory turbulent boundary layer. J. Hydraul. Res., 14, 45-60.

Kajiura, K., 1968. A model of the bottom boundary layer in water waves. Bulletin of the Earthquake Research Institute, 46, 75-123.

Kundu, P. K., J. O. Blanton, and M. M. Janopaul, 1981. Analysis of current observations on the Georgian shelf. Journal of Physical Oceanography, 11, 1139-1149.

Lentz, S. J., and B. Butman, 1995. Comparison of BASS and VACM current measurements during STRESS. Journal of Atmospheric and Oceanic Technology, 12, 1328-1337.

Loder, J. W., 1980. Topographic rectification of tidal currents on the sides of Georges Bank. Journal of Physical Oceanography, 10, 1399-1416.

Maas, L. R. M., and J. J. M. van Haren, 1987. Observations on the vertical structure of tidal and inertial currents in the central North Sea. Journal of Marine Research, 45, 293-318.

Mellor, G. L., and T. Yamada, 1974. A hierarchy of turbulence closure models for planetary boundary layers. Journal of the Atmospheric Sciences, 33, 1791-1896. 
Mellor, G. L., and T. Yamada, 1982. Development of a turbulence closure model for geophysical fluid problems. Reviews of Geophysical and Space Physics, 20, 851-875.

Mofjeld, H. O., 1980. Effects of vertical viscosity on Kelvin waves. Journal of Physical Oceanography, 10, 1039-1050.

Ostendorf, D. W., 1984. The rotary bottom boundary layer. Journal of Geophysical Research, 89 (C6), 10461-10467.

Prandle, D., 1982a. The vertical structure of tidal currents. Geophysical and Astrophysical Fluid Dynamics, 22, 29-49.

Prandle, D., 1982b. The vertical structure of tidal currents and other oscillatory flows. Continental Shelf Research, 1 (2), 191-207.

Soulsby. R. L., and K. R. Dyer, 1981. The form of the near-bed velocity profile in a tidally accelerating flow. Journal of Geophysical Research, 86, 8067-8074.

Soulsby, R. L., 1983. The bottom boundary layer of shelf seas. Physical Oceanography of Coastal and Shelf Seas, B. Johns, editor, Elsevier, Amsterdam, pp. 189-266.

Soulsby, R. L., 1990. Tidal-current boundary layers. The Sea, 9, 523-566.

Sverdrup, H. U., 1927. Dynamics of tides on the north Siberian shelf. Geofys. Publ. Norske Videns Akad., 4, Oslo, Norway.

Tennekes, H., 1973. The logarithmic wind profile. Journal of the Atmospheric Sciences, 30, 234-238.

Trowbridge, J., and O. S. Madsen, 1984. Turbulent wave boundary layers 1. Model formulation and first-order solution. Journal of Geophysical Research, bf 89, 7989-7997.

Uchupi, E., and J. A. Austin, 1987. Morphology. In: Georges Bank, R. H. Backus, editor, The MIT Press, pp. 25-29.

Williams, A. J., 3rd, J. S. Tochko, R.L. Koehler, W. D. Grant, T. F. Gross, and C. V. R. Dunn, 1987. Measurement of turbulence in the oceanic bottom boundary layer with an acoustic current meter array. Journal of Atmospheric and Oceanic Technology, 4(2), 312-327.

Zimmermann, J.T.F., 1980. Vorticity transfer by tidal currents over an irregular topography. Journal of Marine Research, bf 38, 601-630. 


\section{List of Figures}

Figure 1: Bathymetry (in meters) of the southern New England continental margin (Uchupi and Austin, 1987), and geographic location of Stratification Site 1 (ST1).

Figure 2: GLOBEC array (Cruise Report EN260, 1995).

Figure 3: Schematic of the WHOI BASS tripod deployed at ST1 (Cruise Report EN260, 1995).

Figure 4: Temperature difference $\Delta T$ between BASS thermistors 8 and 2 at 5.72 and $0.62 \mathrm{~m}$ above the bottom.

Figure 5: Time series of wind speeds (upper) and wind directions (lower) from IMET data taken at $3.5 \mathrm{~m}$ above sea level. Winds are to the east at $0^{\circ}$, and to the north at $90^{\circ}$.

Figure 6a: Time series of temperature $T$ from Seacat data taken at $10.90 \mathrm{~m}$ (i), $29.40 \mathrm{~m}$ (ii), $49.90 \mathrm{~m}$ (iii), $64.95 \mathrm{~m}$ (iv), $69.75 \mathrm{~m}$ (v), and $74.50 \mathrm{~m}$ (vi) above the bottom.

Figure 6b: Time series of salinity $S$ from Seacat data taken at $10.90 \mathrm{~m}$ (i),

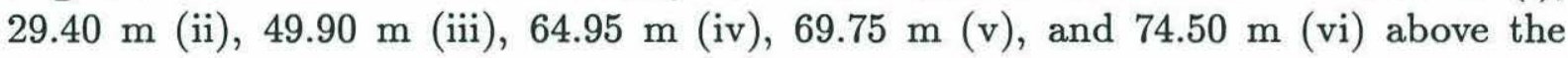
bottom.

Figure 6c: Time series of potential density $\sigma_{\theta}$ from Seacat data taken at $10.90 \mathrm{~m}$ (i), $29.40 \mathrm{~m}$ (ii), $49.90 \mathrm{~m}$ (iii), $64.95 \mathrm{~m}$ (iv), $69.75 \mathrm{~m}$ (v), and $74.50 \mathrm{~m}$ (vi) above the bottom.

Figure 7: Temperature-salinity (T-S) characteristics from Seacat data taken at $10.90 \mathrm{~m}$ (i), $29.40 \mathrm{~m}$ (ii), $49.90 \mathrm{~m}$ (iii), $64.95 \mathrm{~m}$ (iv), $69.75 \mathrm{~m} \mathrm{(v),} \mathrm{and} 74.50 \mathrm{~m}$ (vi) above the bottom.

Figure 8: Profiles of mean temperature $\mathrm{T}$ (a), salinity $\mathrm{S}$ (b), and potential density $\sigma_{\theta}$ (c) from Seacat data for the time period from yearday 50 to 80 . Dashed lines represent mean values obtained after isolated events of high stratification near the surface have been excluded.

Figure 9: Differences in measured speeds $\Delta U$ between pods 5 and 4 (a), 4 and 3 (b), 3 and 2 (c), and 2 and 1 (d) as a function of mean flow direction at pods 1-5. Flow orientations are eastward at $0^{\circ}$, and northward at $90^{\circ}$. Solid lines represent directions 
where flow obstruction due to the tripod legs is possible. Dashed and dotted lines mark the orientation of the major and minor axes of the $\mathrm{M}_{2}$ current ellipse, respectively.

Figure 10: Averaged BASS velocity profiles taken between yearday 50-80 within $\pm 5^{\circ}$ from angles of largest increase and decrease of tidal current speeds, $U$. Profiles are displayed on a semi-logarithmic (base 10) scale. Dashed lines represent results from a least-squares logarithmic fit to measured current speeds at pods $1-4 . \frac{\partial U}{\partial t}$ is largest around $-20^{\circ}$ (a) and $160^{\circ}(\mathrm{c})$, and smallest around $-110^{\circ}(\mathrm{b})$ and $70^{\circ}(\mathrm{d})$, where minus signs denote clockwise from east.

Figure 11: Averaged BASS velocity profiles taken between yearday 50-80 within $\pm 5^{\circ}$ from angles of largest increase and decrease of tidal current speeds, $U$, with their offset residuals being removed. Profiles are displayed on a semi-logarithmic (base 10) scale. Dashed lines represent results from a least-squares logarithmic fit to measured current speeds at pods $1-4 . \frac{\partial U}{\partial t}$ is largest around $-20^{\circ}$ (a) and $160^{\circ}(\mathrm{c})$, and smallest around $-110^{\circ}(\mathrm{b})$ and $70^{\circ}(\mathrm{d})$, where minus signs denote clockwise from east.

Figure 12: Time series of friction velocities $u_{*}$ obtained from best-fit logarithmic profiles to BASS velocity data.

Figure 13: Estimated friction velocities $u_{*}$ (a), and ratios $\frac{\Delta u_{*}^{95}}{u_{*}^{9}}$ (b) versus speed at pod 3, where $\Delta u_{*}^{95}$ is the $95 \%$ confidence interval of $u_{*}$.

Figure 14: Estimated bottom roughness $z_{0}$ (a), ratios $\frac{\Delta z_{0}^{95+}}{z_{0}}$ (b), and ratios $\frac{\Delta z_{0}^{95}}{z_{0}}$ (c) versus $u_{*}$, where $\Delta z_{0}^{95+}$ and $\Delta z_{0}^{95-}$ are the upper and lower $95 \%$ confidence intervals of $z_{0}$, respectively. Bottom roughness estimates (a) are displayed on a semi-logarithmic (base 10) scale.

Figure 15: Picture of the sea floor displaying the presence of sand ripples. Cables in the lower left corner are connected to the camera, and are $\approx 40^{\circ}$ counterclockwise from east. Sand ripples are aligned north eastward.

Figure 16: Histograms of bottom roughness $\log _{10}\left(z_{0}\right)$, where $z_{0}$ is in millimeters. All flow directions are included in (a), while directions believed to be affected by the battery case $\left(35^{\circ}-75^{\circ}\right.$ clockwise from east) are excluded in (b).

Figure 17: Estimates of bottom roughness $z_{0}$ versus ratios $\frac{u_{b}}{U_{1}}$ on a logarithmic (base 10) scale. $U_{1}$ is the measured current speed at pod 1, and $u_{b}$ is the orbital wave velocity near the bottom. 
Figure 18: Vertical structure of the $M_{2}$ tidal velocities (solid lines) and total current speeds (dotted lines) from BASS and VMCM data taken between yearday 50-80. Profiles are displayed on a semi-logarithmic (base 10) scale. Dashed lines represent results from a least-squares logarithmic fit to measured current speeds at pods 1-4. Profiles are from averaged data, where the average was taken of current measurements with flow directions less than $\pm 5^{\circ}$ from angles of maximum acceleration and deceleration of the near surface tidal speeds $U . \frac{\partial U}{\partial t}$ is largest around $-14^{\circ}$ (a) and $166^{\circ}$ (c), and smallest around $-104^{\circ}(\mathrm{b})$ and $76^{\circ}(\mathrm{d})$, where minus signs denote clockwise from east.

Figure 19: Time series of depth-averaged, low-pass filtered flow speeds (upper) and flow directions (lower). The low-pass filter applies to frequencies smaller than diurnal. Flow orientations are eastward at $0^{\circ}$, and northward at $90^{\circ}$.

Figure 20: Vertical structure of the $M_{2}$ current ellipse obtained from tidal analysis of BASS and VMCM data for yearday 50-80. Profiles show the magnitudes of the rotary components $R^{+}$(solid line) and $R^{-}$(dashed line) (a), corresponding phase angles $\phi^{+}$(b) and $\phi^{-}$(c), current amplitudes $U_{M a j}$ and $U_{M i n}(\mathrm{~d})$, inclination of the major axis $\theta$ (e), and phase of the velocity vector $\phi(f)$. Dotted lines represent error bars. Inclination and phase angles of the rotary components are given with respect to east, where negative values are clockwise from east. Phase angles describe the orientation of the $M_{2}$ velocity vector at the beginning of the time series.

Figure 21: Kinetic energy spectrum of wind speeds measured by the IMET between yearday $50-80$.

Figure 22: Vertical structure of the $\mathrm{N}_{2}$ current ellipse obtained from tidal analysis of BASS and VMCM data for yearday 50-80. Profiles show the magnitudes of the rotary components $R^{+}$(solid line) and $R^{-}$(dashed line) (a), corresponding phase angles $\phi^{+}$(b) and $\phi^{-}$(c), current amplitudes $U_{M a j}$ and $U_{M i n}(\mathrm{~d})$, inclination of the major axis $\theta$ (e), and phase of the velocity vector $\phi(f)$. Dotted lines represent error bars. Inclination and phase angles of the rotary components are given with respect to east, where negative values are clockwise from east. Phase angles describe the orientation of the $\mathrm{N}_{2}$ velocity vector at the beginning of the time series.

Figure 23: Vertical structure of the $S_{2}$ current ellipse obtained from tidal analysis of BASS and VMCM data for yearday 50-80. Profiles show the magnitudes of the rotary components $R^{+}$(solid line) and $R^{-}$(dashed line) (a), corresponding phase angles $\phi^{+}$(b) and $\phi^{-}$(c), current amplitudes $U_{M a j}$ and $U_{M i n}(\mathrm{~d})$, inclination of the major axis $\theta(\mathrm{e})$, and phase of the velocity vector $\phi(\mathrm{f})$. Dotted lines represent error bars. Inclination and phase angles of the rotary components are given with respect to east, 
where negative values are clockwise from east. Phase angles describe the orientation of the $S_{2}$ velocity vector at the beginning of the time series.

Figure 24: Vertical structure of the $K_{1}$ current ellipse obtained from tidal analysis of BASS and VMCM data for yearday 50-80. Profiles show the magnitudes of the rotary components $R^{+}$(solid line) and $R^{-}$(dashed line) (a), corresponding phase angles $\phi^{+}(\mathrm{b})$ and $\phi^{-}(\mathrm{c})$, current amplitudes $U_{M a j}$ and $U_{M i n}(\mathrm{~d})$, inclination of the major axis $\theta$ (e), and phase of the velocity vector $\phi(f)$. Dotted lines represent error bars. Inclination and phase angles of the rotary components are given with respect to east, where negative values are clockwise from east. Phase angles describe the orientation of the $K_{1}$ velocity vector at the beginning of the time series.

Figure 25: Profiles of the $M_{2}$ stress ellipse obtained from vertical integration of the momentum equations, using the BASS bottom stress estimates as a lower boundary condition. Profiles show the magnitudes of the rotary components $\tau^{+}$(solid line) and $\tau^{-}$(dashed line) (a), phase angles $\phi_{\tau}^{+}(\mathrm{b})$ and $\phi_{\tau}^{-}(\mathrm{c})$, current amplitudes $\tau_{M a j}$ and $\tau_{\text {Min }}(\mathrm{d})$, inclination of the major axis $\theta_{\tau}(\mathrm{e})$, and phase of the stress vector $\phi_{\tau}(\mathrm{f})$. Dotted lines represent error bars. Inclination and phase angles of the rotary components are given with respect to east, where negative values are clockwise from east. Phase angles describe the orientation of the $\mathrm{M}_{2}$ stress vector at the beginning of the time series.

Figure 26: Profiles of the $M_{2}$ stress ellipse parameters obtained from vertical integration of the velocity defect, assuming a region of zero stress divergence at heights z $35 \mathrm{~m}$. Dash-dotted lines correspond to results displayed in Figure 25. Profiles show the magnitudes of the rotary components $\tau^{+}$(solid line) and $\tau^{-}$(dashed line) (a), phase angles $\phi_{\tau}^{+}(\mathrm{b})$ and $\phi_{\tau}^{-}(\mathrm{c})$, current amplitudes $\tau_{M a j}$ and $\tau_{M i n}(\mathrm{~d})$, inclination of the major axis $\theta_{\tau}(\mathrm{e})$, and phase of the stress vector $\phi_{\tau}(\mathrm{f})$. Dotted lines represent error bars associated with the velocity defect method. Inclination and phase angles of the rotary components are given with respect to east, where negative values are clockwise from east. Phase angles describe the orientation of the $M_{2}$ stress vector at the beginning of the time series.

Figure 27: Vertical structure of the integrated velocity defect for the $M_{2}$ constituent (solid lines), and boundary layer transports predicted by $\mathrm{M}_{2}$ bottom stress estimates obtained from BASS data (dashed lines). Profiles show transport magnitudes $T^{+}$(a) and $T^{-}$(b), and corresponding phase angles $\phi_{T}^{+}$(c) and $\phi_{T}^{-}$(d). Dotted lines are error bars. Phase angles describe the orientation of the $\mathrm{M}_{2}$ transport vector at the beginning of the time series and are given with respect to east, where negative values are clockwise from east. 
Figure 28: Vertical structure of mean eddy viscosities $K$ calculated for yearday 5080. Profiles show the average hourly variation of $K$ during one tidal cycle (a), and the vertical structure of the time mean (b and c). Dotted lines in ( $b$ and $c$ ) are error bars. Dashed lines in (b and c) depict the slope $\kappa \bar{u}_{*} z$, where $\bar{u}_{*}=1.2 \mathrm{~cm} / \mathrm{s}$ according to estimates derived from BASS data.

Figure 29: Vertical structure of the total current speeds $U$ from BASS and VMCM data taken between yearday 50-80. Dashed lines represent results from a least-squares logarithmic fit to velocity data at pods $1-4$. Profiles are from averaged data, where the average was taken of current measurements with flow directions less than $\pm 5^{\circ}$ from angles of maximum acceleration and deceleration of the near surface tidal speeds. $\frac{\partial U}{\partial t}$ is largest around $-14^{\circ}$ (a) and $166^{\circ}(\mathrm{c})$, and smallest around $-104^{\circ}(\mathrm{b})$ and $76^{\circ}(\mathrm{d})$, where minus signs denote clockwise from east.

Figure 30: Model results for the vertical structure of the $M_{2}$ current ellipse obtained from the K-profile model with sublayer height $l=\frac{1}{6} \cdot \frac{0.4 \bar{u}_{*}}{\sigma-f}$. Circles represent results obtained from BASS and VMCM measurements, and dotted lines depict the associated uncertainties (Figure 20). Profiles show the magnitudes of the rotary components $R^{+}$(solid line) and $R^{-}$(dashed line) (a), relative veering of the corresponding phase angles $\Delta \phi^{+}$(c) and $\Delta \phi^{-}$(d), current amplitudes $U_{M a j}$ and $U_{M i n}$ (d), relative veering of the inclination of the major axis $\Delta \theta$ (e) and of the phase of the velocity vector $\Delta \phi(f)$. Decreasing angles $\Delta \theta$ and $\Delta \phi^{ \pm}$depict clockwise rotation of the major axis $U_{M a j}$ and rotary components $R^{ \pm}$, respectively. Decreasing angles $\Delta \phi$ represent phase lag.

Figure 31: Numerical eddy viscosities $K$ obtained from the $\mathrm{K}$-profile model with sublayer height $l=\frac{1}{6} \cdot \frac{0.4 \bar{u}_{*}}{\sigma-f}$ (solid line). Circles represent results from measured data, and dotted lines are the corresponding uncertainties (Figure 28). In the sublayer, $K=\kappa \bar{u}_{*} z$, where $\bar{u}_{*}=1.45 \mathrm{~cm} / \mathrm{s}$ according to model results.

Figure 32: Model results for the vertical structure of the $\mathrm{M}_{2}$ stress ellipse obtained from the K-profile model with sublayer height $l=\frac{1}{6} \cdot \frac{0.4 \bar{u}_{*}}{\sigma-f}$. Circles represent results from actual data, and dotted lines are the associated uncertainties (Figure 25). Profiles show the magnitudes of the rotary components $\tau^{+}$(solid line) and $\tau^{-}$(dashed line) (a), corresponding phase angles $\phi_{\tau}^{+}(\mathrm{c})$ and $\phi_{\tau}^{-}(\mathrm{d})$, current amplitudes $\tau_{M a j}$ and $\tau_{\text {Min }}(\mathrm{d})$, inclination of the major axis $\theta_{\tau}(\mathrm{e})$, and phase of the stress vector $\phi_{\tau}(\mathrm{f})$. Inclination and phase angles of the rotary components are given with respect to east, where negative values are clockwise from east. Phase angles describe the orientation of the $M_{2}$ stress vector at the beginning of the time series. 
Figure 33: Model results for the integrated velocity defect of the $M_{2}$ constituent (solid lines), and tidal boundary layer transports predicted by numerical bottom stresses (dotdashed lines) obtained from the K-profile model with sublayer height $l=\frac{1}{6} \cdot \frac{0.4 \bar{u}_{*}}{\sigma-f}$. Circles represent results from velocity defects of actual data, and dashed lines are boundary layer transports predicted by BASS bottom stress estimates (Figure 27). Dotted lines are uncertainties associated with the data. Profiles show transport magnitudes $T^{+}$(a) and $T^{-}$(b), and corresponding phase angles $\phi_{T}^{+}$(c) and $\phi_{T}^{-}$(d). Phase angles describe the orientation of the $\mathrm{M}_{2}$ transport vector at the beginning of the time series and are given with respect to east, where negative values are clockwise from east.

Figure 34: Model results for the vertical structure of the $M_{2}$ current ellipse obtained from the K-profile model with sublayer height $l=\frac{1}{6} \cdot 0.39 \frac{\kappa \bar{u}_{*}}{\sigma-f}$. Circles represent results obtained from BASS and VMCM measurements, and dotted lines depict the associated uncertainties (Figure 20). Profiles show the magnitudes of the rotary components $R^{+}$(solid line) and $R^{-}$(dashed line) (a), relative veering of the corresponding phase angles $\Delta \phi^{+}$(c) and $\Delta \phi^{-}$(d), current amplitudes $U_{M a j}$ and $U_{M i n}$ (d), relative veering of the inclination of the major axis $\Delta \theta$ (e) and of the phase of the velocity vector $\Delta \phi(f)$. Decreasing angles $\Delta \theta$ and $\Delta \phi^{ \pm}$depict clockwise rotation of the major axis $U_{M a j}$ and rotary components $R^{ \pm}$, respectively. Decreasing angles $\Delta \phi$ represent phase lag.

Figure 35: Numerical eddy viscosities $K$ obtained from the $\mathrm{K}$-profile model with sublayer height $l=\frac{1}{6} \cdot 0.39 \frac{\kappa \bar{u}_{*}}{\sigma-f}$ (solid line). Circles represent results from measured data, and dotted lines are the corresponding uncertainties (Figure 28). In the sublayer, $K=\kappa \bar{u}_{*} z$, where $\bar{u}_{*}=1.45 \mathrm{~cm} / \mathrm{s}$ according to model results.

Figure 36: Model results for the vertical structure of the $M_{2}$ stress ellipse obtained from the K-profile model with sublayer height $l=\frac{1}{6} \cdot 0.39 \frac{\kappa \bar{u}_{*}}{\sigma-f}$. Circles represent results from actual data, and dotted lines are the associated uncertainties (Figure 25). Profiles show the magnitudes of the rotary components $\tau^{+}$(solid line) and $\tau^{-}$(dashed line) (a), corresponding phase angles $\phi_{\tau}^{+}(\mathrm{c})$ and $\phi_{\tau}^{-}(\mathrm{d})$, current amplitudes $\tau_{M a j}$ and $\tau_{\text {Min }}(\mathrm{d})$, inclination of the major axis $\theta_{\tau}(\mathrm{e})$, and phase of the stress vector $\phi_{\tau}(\mathrm{f})$. Inclination and phase angles of the rotary components are given with respect to east, where negative values are clockwise from east. Phase angles describe the orientation of the $M_{2}$ stress vector at the beginning of the time series.

Figure 37: Model results for the integrated velocity defect of the $M_{2}$ constituent (solid lines), and tidal boundary layer transports predicted by numerical bottom stresses (dotdashed lines) obtained from the K-profile model with sublayer height $l=\frac{1}{6} \cdot 0.39 \frac{\kappa \bar{u}_{s}}{\sigma-f}$. 
Circles represent results from velocity defects of actual data, and dashed lines are boundary layer transports predicted by BASS bottom stress estimates (Figure 27). Dotted lines are uncertainties associated with the data. Profiles show transport magnitudes $T^{+}$(a) and $T^{-}$(b), and corresponding phase angles $\phi_{T}^{+}$(c) and $\phi_{T}^{-}$(d). Phase angles describe the orientation of the $\mathrm{M}_{2}$ transport vector at the beginning of the time series and are given with respect to east, where negative values are clockwise from east.

Figure 38: Model results for the vertical structure of the $M_{2}$ current ellipse obtained from the K-profile model with sublayer height $l=\frac{1}{10} \cdot 0.39 \frac{\kappa \bar{u}_{*}}{\sigma-f}$. Circles represent results obtained from BASS and VMCM measurements, and dotted lines depict the associated uncertainties (Figure 20). Profiles show the magnitudes of the rotary components $R^{+}$(solid line) and $R^{-}$(dashed line) (a), relative veering of the corresponding phase angles $\Delta \phi^{+}$(c) and $\Delta \phi^{-}$(d), current amplitudes $U_{M a j}$ and $U_{M i n}$ (d), relative veering of the inclination of the major axis $\Delta \theta$ (e) and of the phase of the velocity vector $\Delta \phi(f)$. Decreasing angles $\Delta \theta$ and $\Delta \phi^{ \pm}$depict clockwise rotation of the major axis $U_{M a j}$ and rotary components $R^{ \pm}$, respectively. Decreasing angles $\Delta \phi$ represent phase lag.

Figure 39: Numerical eddy viscosities $K$ obtained from the $\mathrm{K}$-profile model with sublayer height $l=\frac{1}{10} \cdot 0.39 \frac{\kappa \bar{u}_{*}}{\sigma-f}$ (solid line). Circles represent results from measured data, and dotted lines are the corresponding uncertainties (Figure 28). In the sublayer, $K=\kappa \bar{u}_{*} z$, where $\bar{u}_{*}=1.45 \mathrm{~cm} / \mathrm{s}$ according to model results.

Figure 40: Model results for the vertical structure of the $\mathrm{M}_{2}$ stress ellipse obtained from the K-profile model with sublayer height $l=\frac{1}{10} \cdot 0.39 \frac{\kappa \bar{u}_{*}}{\sigma-f}$. Circles represent results from actual data, and dotted lines are the associated uncertainties (Figure 25). Profiles show the magnitudes of the rotary components $\tau^{+}$(solid line) and $\tau^{-}$(dashed line) (a), corresponding phase angles $\phi_{\tau}^{+}(\mathrm{c})$ and $\phi_{\tau}^{-}(\mathrm{d})$, current amplitudes $\tau_{M a j}$ and $\tau_{\text {Min }}(\mathrm{d})$, inclination of the major axis $\theta_{\tau}(\mathrm{e})$, and phase of the stress vector $\phi_{\tau}(\mathrm{f})$. Inclination and phase angles of the rotary components are given with respect to east, where negative values are clockwise from east. Phase angles describe the orientation of the $\mathrm{M}_{2}$ stress vector at the beginning of the time series.

Figure 41: Model results for the integrated velocity defect of the $M_{2}$ constituent (solid lines), and tidal boundary layer transports predicted by numerical bottom stresses (dotdashed lines) obtained from the K-profile model with sublayer height $l=\frac{1}{10} \cdot 0.39 \frac{\kappa \bar{u}_{*}}{\sigma-f}$. Circles represent results from velocity defects of actual data, and dashed lines are boundary layer transports predicted by BASS bottom stress estimates (Figure 27). Dotted lines are uncertainties associated with the data. Profiles show transport mag- 
nitudes $T^{+}$(a) and $T^{-}$(b), and corresponding phase angles $\phi_{T}^{+}$(c) and $\phi_{T}^{-}$(d). Phase angles describe the orientation of the $\mathrm{M}_{2}$ transport vector at the beginning of the time series and are given with respect to east, where negative values are clockwise from east.

Figure 42: Model results for the vertical structure of the $M_{2}$ current ellipse obtained from the MY 2.5. Circles represent results obtained from BASS and VMCM measurements, and dotted lines depict the associated uncertainties (Figure 20). Profiles show the magnitudes of the rotary components $R^{+}$(solid line) and $R^{-}$(dashed line) (a), relative veering of the corresponding phase angles $\Delta \phi^{+}$(c) and $\Delta \phi^{-}(\mathrm{d})$, current amplitudes $U_{M a j}$ and $U_{M i n}(\mathrm{~d})$, relative veering of the inclination of the major axis $\Delta \theta$ (e) and of the phase of the velocity vector $\Delta \phi$ (f). Decreasing angles $\Delta \theta$ and $\Delta \phi^{ \pm}$depict clockwise rotation of the major axis $U_{M a j}$ and rotary components $R^{ \pm}$, respectively. Decreasing angles $\Delta \phi$ represent phase lag.

Figure 43: Numerical eddy viscosities $K$ obtained from the MY 2.5. Circles represent results from measured data, and dotted lines are the corresponding uncertainties (Figure 28). In the sublayer, $K=\kappa \bar{u}_{*} z$, where $\bar{u}_{*}=1.4 \mathrm{~cm} / \mathrm{s}$ according to model results.

Figure 44: Model results for the vertical structure of the $M_{2}$ stress ellipse obtained from the MY 2.5. Circles represent results from actual data, and dotted lines are the associated uncertainties (Figure 25). Profiles show the magnitudes of the rotary components $\tau^{+}$(solid line) and $\tau^{-}$(dashed line) (a), corresponding phase angles $\phi_{\tau}^{+}$(c) and $\phi_{\tau}^{-}(\mathrm{d})$, current amplitudes $\tau_{M a j}$ and $\tau_{M i n}(\mathrm{~d})$, inclination of the major axis $\theta_{\tau}(\mathrm{e})$, and phase of the stress vector $\phi_{\tau}(\mathrm{f})$. Inclination and phase angles of the rotary components are given with respect to east, where negative values are clockwise from east. Phase angles describe the orientation of the $M_{2}$ stress vector at the beginning of the time series.

Figure 45: Model results for the integrated velocity defect of the $M_{2}$ constituent (solid lines), and tidal boundary layer transports predicted by numerical bottom stresses (dotdashed lines) obtained from the MY 2.5. Circles represent results from velocity defects of actual data, and dashed lines are boundary layer transports predicted by BASS bottom stress estimates (Figure 27). Dotted lines are uncertainties associated with the data. Profiles show transport magnitudes $T^{+}$(a) and $T^{-}$(b), and corresponding phase angles $\phi_{T}^{+}$(c) and $\phi_{T}^{-}$(d). Phase angles describe the orientation of the $\mathrm{M}_{2}$ transport vector at the beginning of the time series and are given with respect to east, where negative values are clockwise from east. 
Figure 46: $\frac{\bar{V}_{\infty}}{\tilde{U}_{0}}$ versus $\frac{\bar{U}_{\infty}}{\tilde{U}_{0}}$, where $\tilde{U}_{\infty}$ and $\tilde{V}_{\infty}$ are characteristic interior subtidal velocities deduced from observations, and representative of times where acceleration of the mean flow as well as horizontal density gradients are small. $\tilde{U}_{0}$ is a characteristic free stream velocity computed under the assumption of barotropic flow and is approximately along-isobath. Dotted lines depict the outer margins of associated error bars.

Figure 47: Velocity profiles of the subtidal current representative of times where acceleration of the mean flow and horizontal density gradients are small. The components $\bar{u}$ (a) and $\bar{v}$ (b) are along-bank and cross-bank, respectively, where the along-bank direction is at $-148^{\circ}$ from east. Profiles are from averaged data, where the average was taken of all nine $12.42 \mathrm{hrs}$-bin events meeting the requirements of quasi-steadiness and small baroclinic pressure forcing. Standard deviations from the mean are shown (c) and (d).

Figure 48: Integrated velocity defects $\bar{T}^{x}$ and $\bar{T}^{y}$ for the along-bank (a) and acrossbank (b) components of the subtidal flow (solid lines), and predicted boundary layer transports - $\frac{\bar{\tau}_{b}^{y}}{\rho_{0} f}$ and $\frac{\bar{\tau}_{o}^{x}}{\rho_{0} f}$ from low-pass filtered bottom stress estimates (dashed lines). Profiles are from averaged data, where the average was taken of all nine $12.42 \mathrm{hrs}-$ bin events meeting the requirements of quasi-steadiness and small baroclinic pressure forcing. Dotted lines depict the standard deviation from the mean. 
FIGURES 


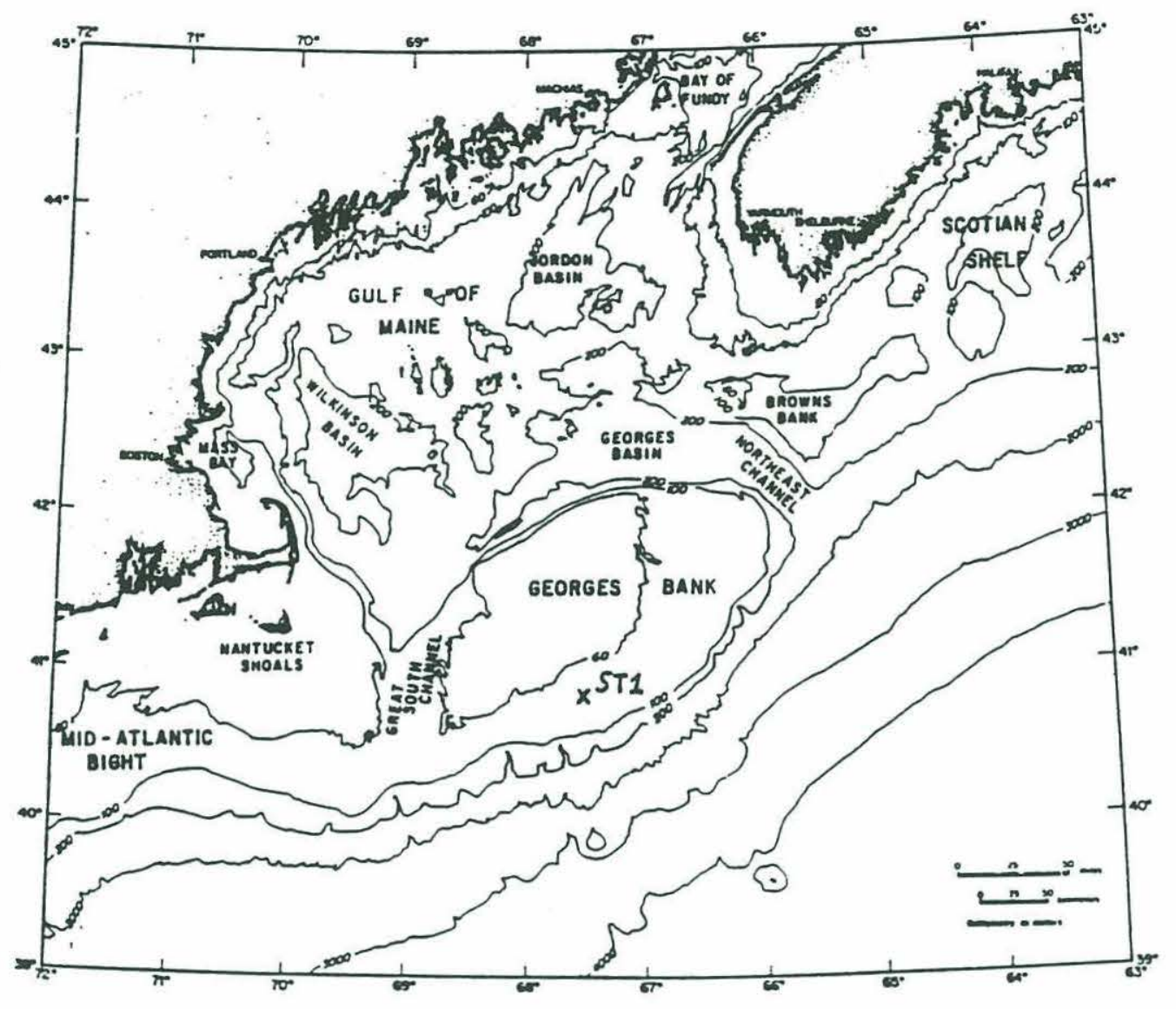

Figure 1: Bathymetry (in meters) of the southern New England continental margin (Uchupi and Austin, 1987), and geographic location of Stratification Site 1 (ST1). 


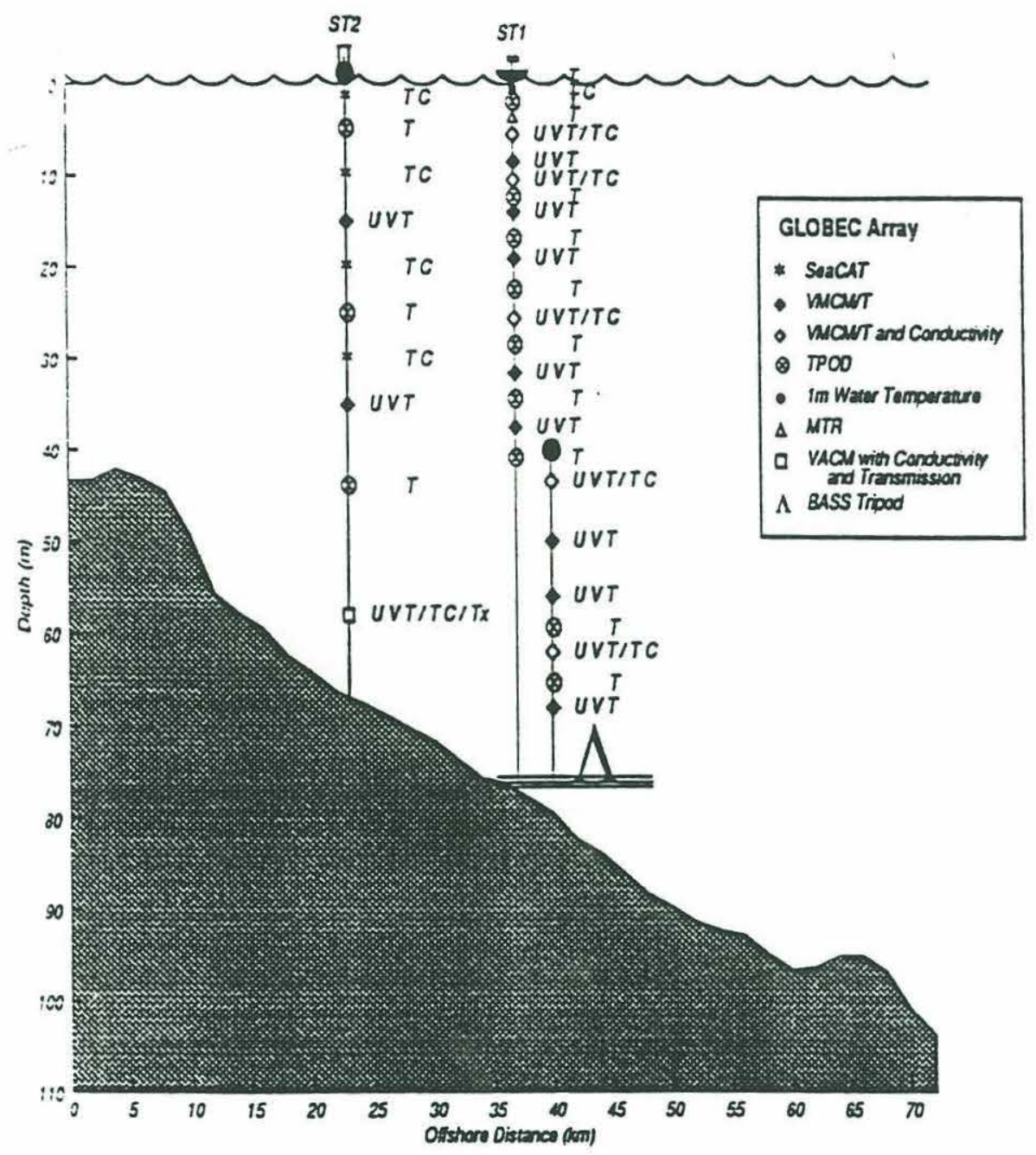

Figure 2: GLOBEC array (Cruise Report EN260, 1995). 


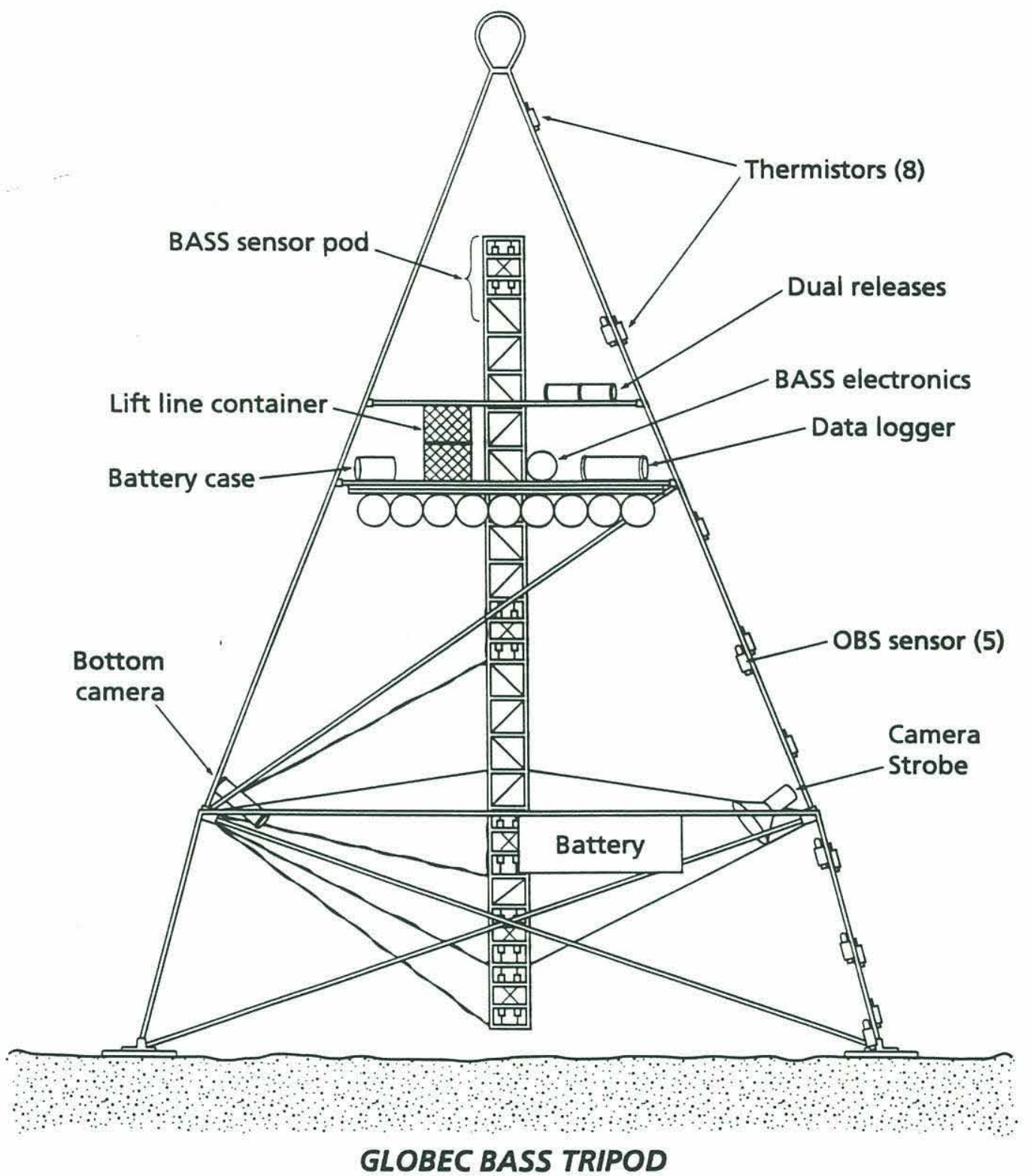

Figure 3: Schematic of the WHOI BASS tripod deployed at ST1 (Cruise Report EN260, 1995). 


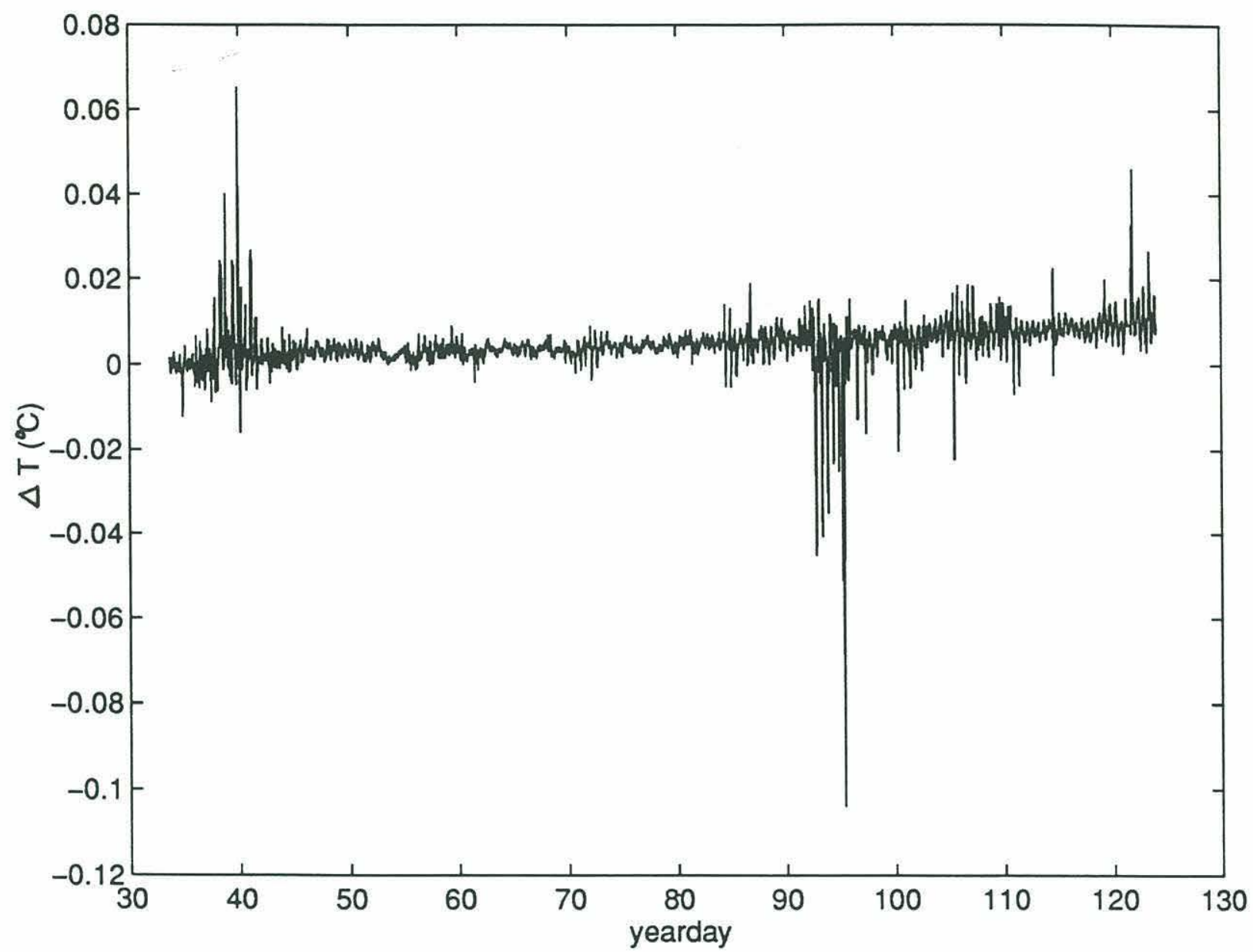

Figure 4: Temperature difference $\Delta T$ between BASS thermistors 8 and 2 at 5.72 and $0.62 \mathrm{~m}$ above the bottom. 

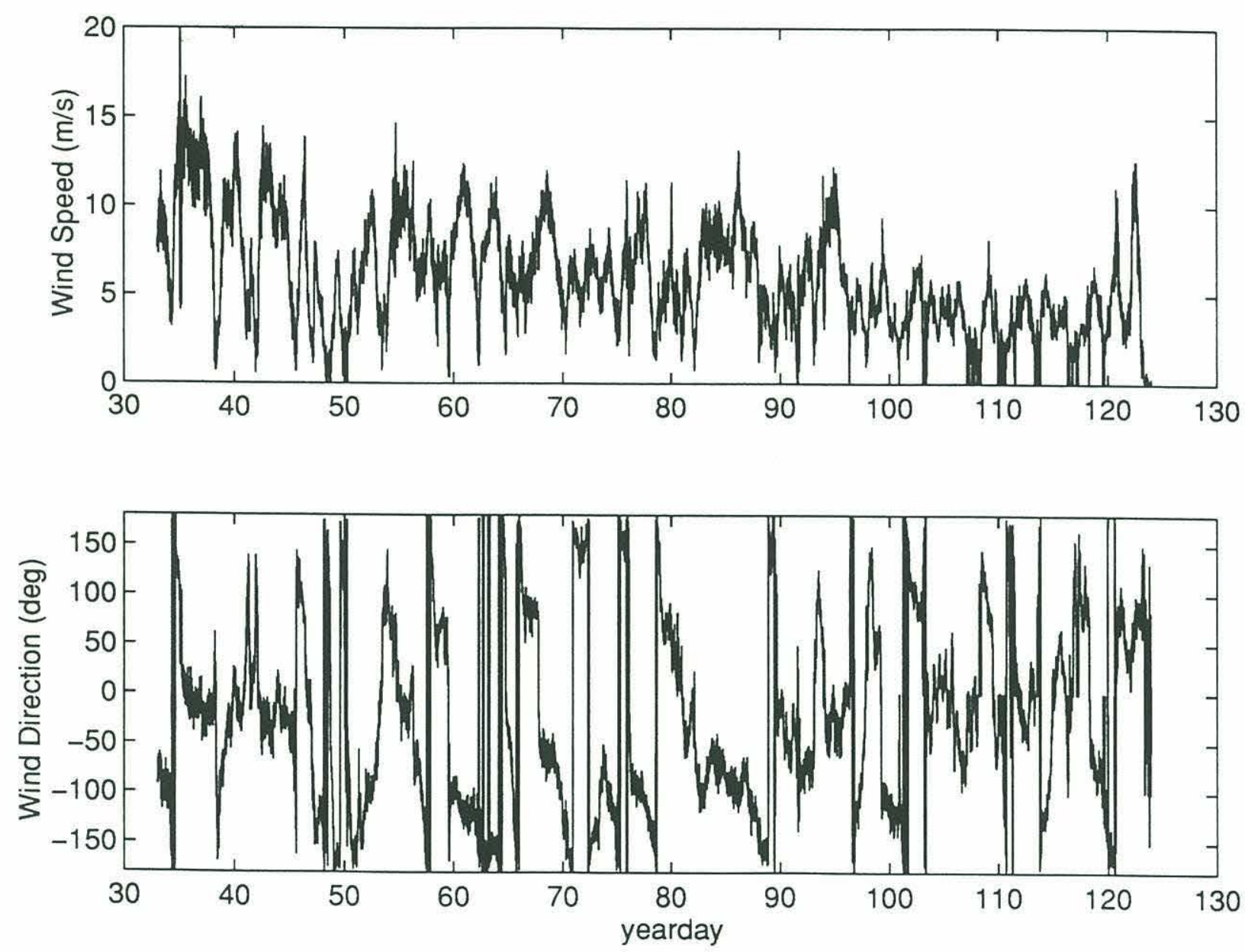

Figure 5: Time series of wind speeds (upper) and wind directions (lower) from IMET data taken at $3.5 \mathrm{~m}$ above sea level. Winds are to the east at $0^{\circ}$, and to the north at $90^{\circ}$. 

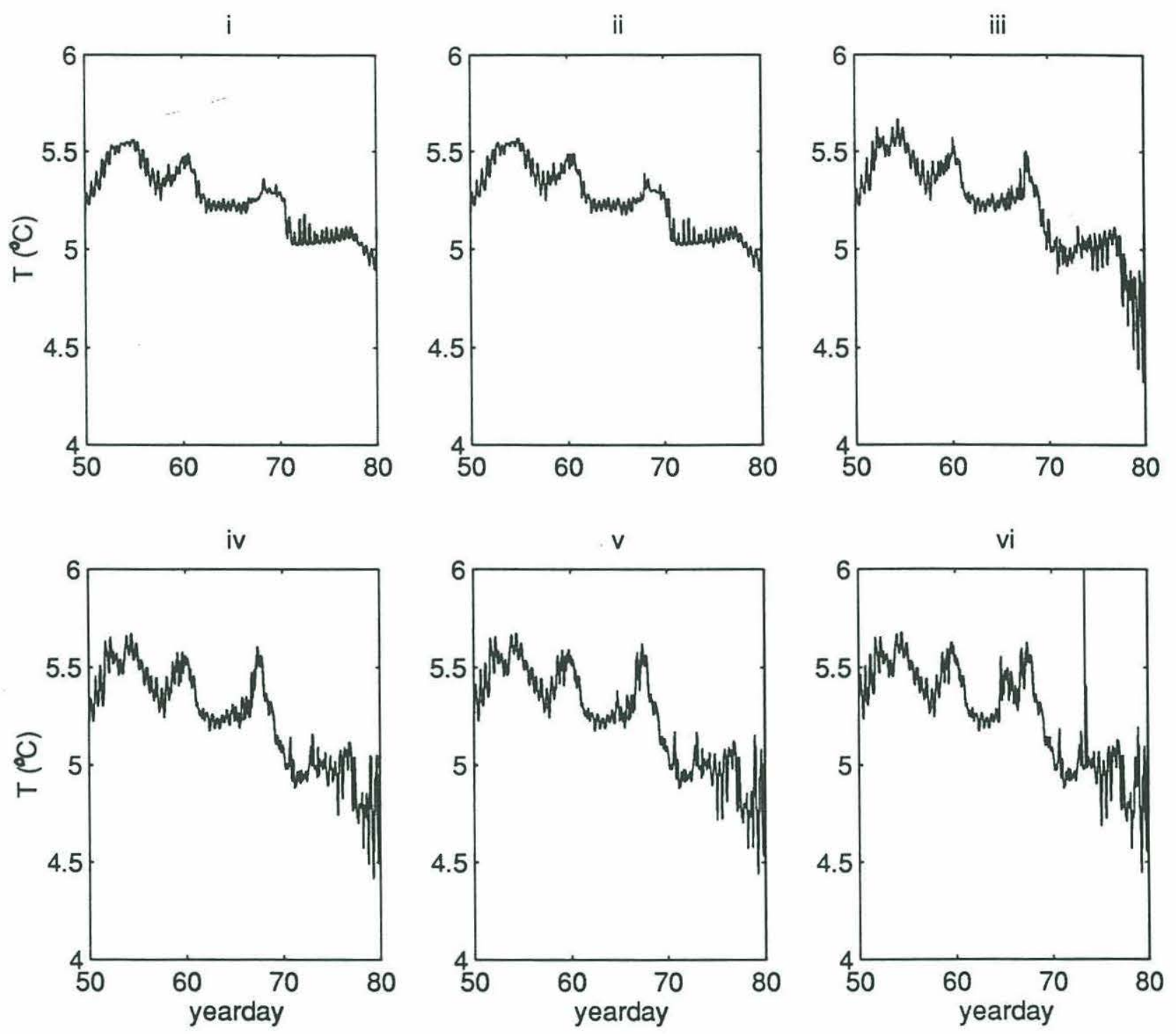

Figure 6a: Time series of temperature $T$ from Seacat data taken at $10.90 \mathrm{~m}$ (i), $29.40 \mathrm{~m}$ (ii), $49.90 \mathrm{~m}$ (iii), $64.95 \mathrm{~m}$ (iv), $69.75 \mathrm{~m}$ (v), and $74.50 \mathrm{~m}$ (vi) above the bottom. 

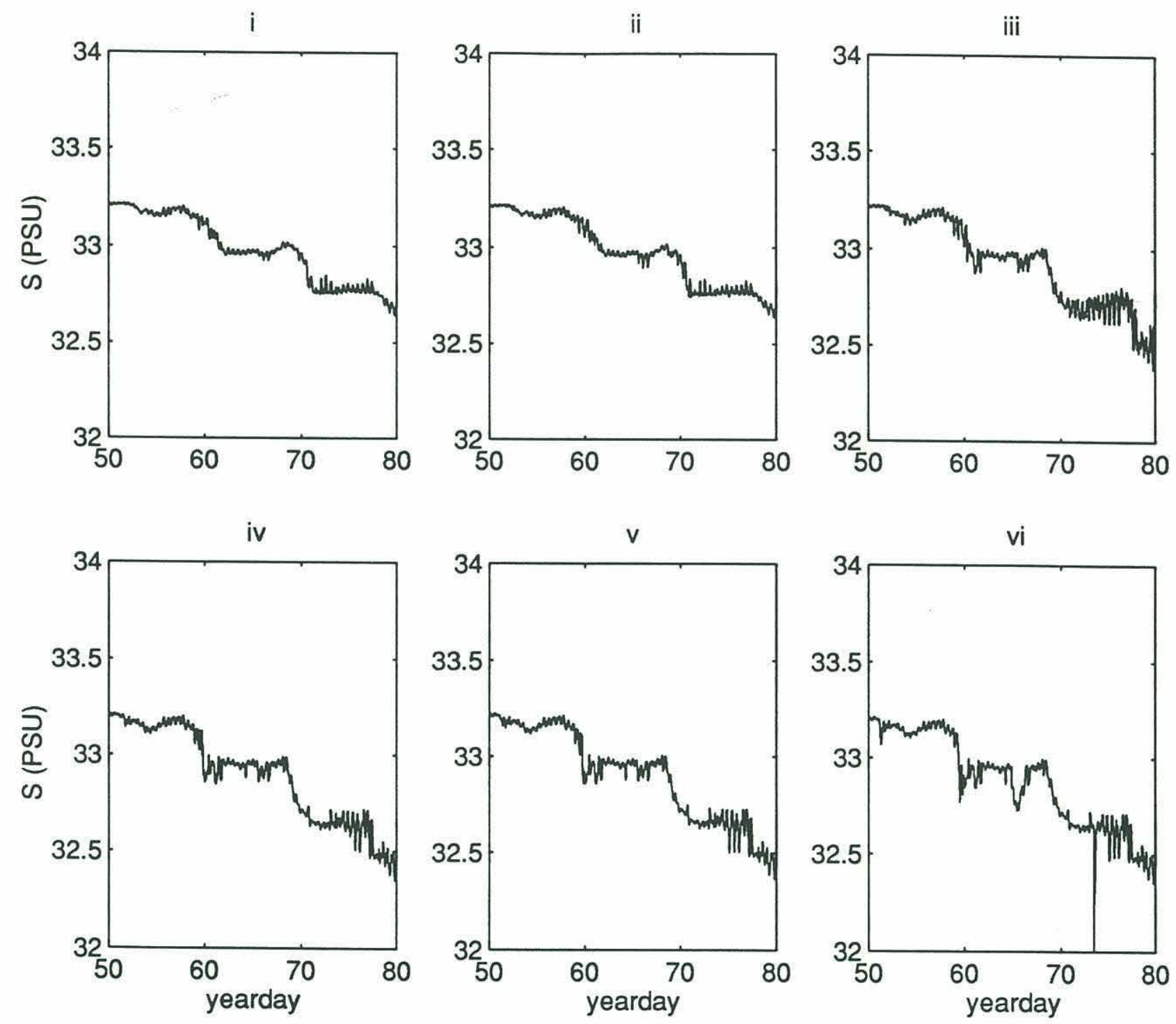

Figure 6b: Time series of salinity $S$ from Seacat data taken at $10.90 \mathrm{~m}$ (i), $29.40 \mathrm{~m}$ (ii), $49.90 \mathrm{~m}$ (iii), $64.95 \mathrm{~m}$ (iv), $69.75 \mathrm{~m}$ (v), and $74.50 \mathrm{~m}$ (vi) above the bottom. 

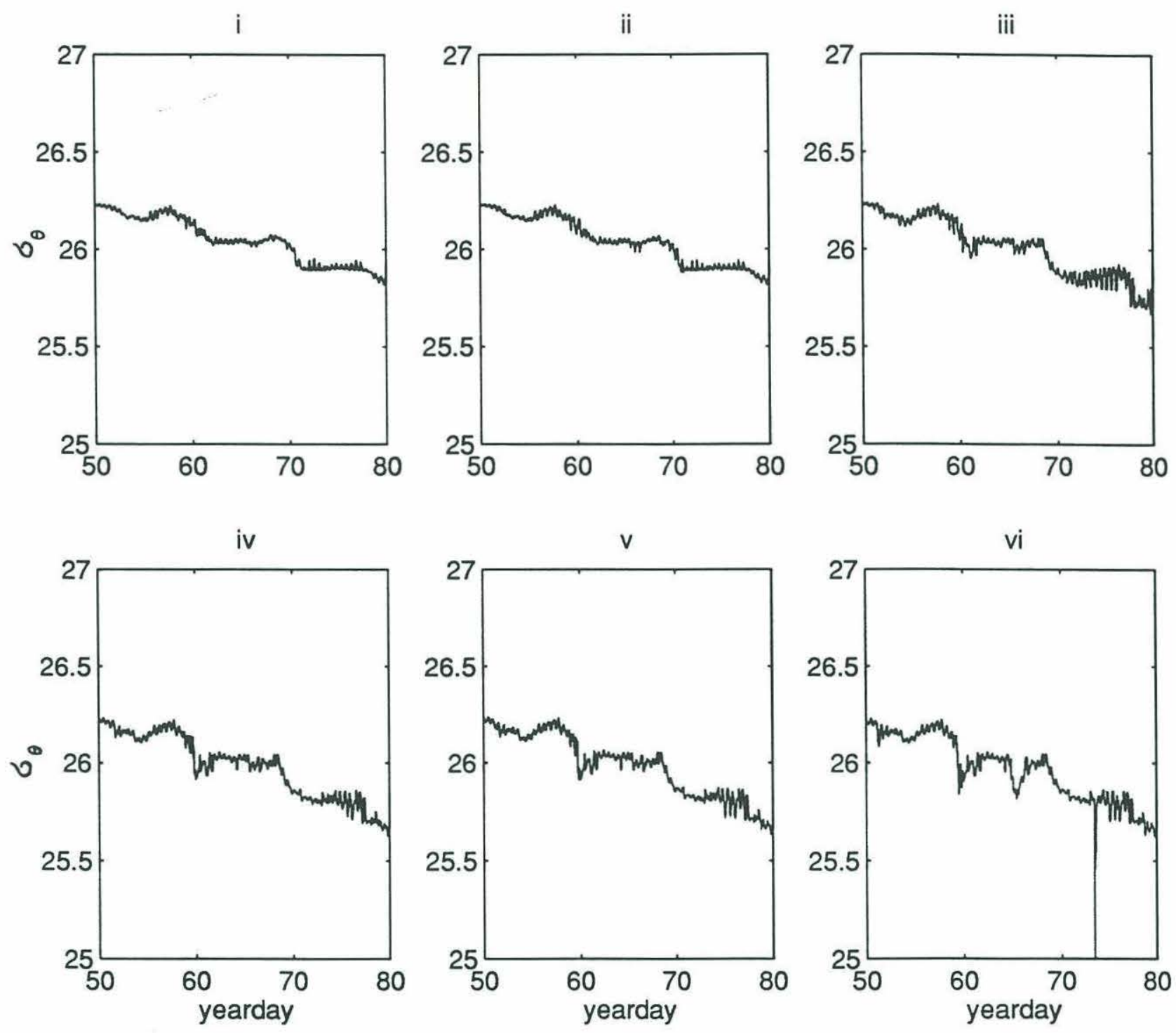

Figure 6c: Time series of potential density $\sigma_{\theta}$ from Seacat data taken at $10.90 \mathrm{~m}$ (i), $29.40 \mathrm{~m}$ (ii), $49.90 \mathrm{~m}$ (iii), $64.95 \mathrm{~m}$ (iv), $69.75 \mathrm{~m}$ (v), and $74.50 \mathrm{~m}$ (vi) above the bottom. 

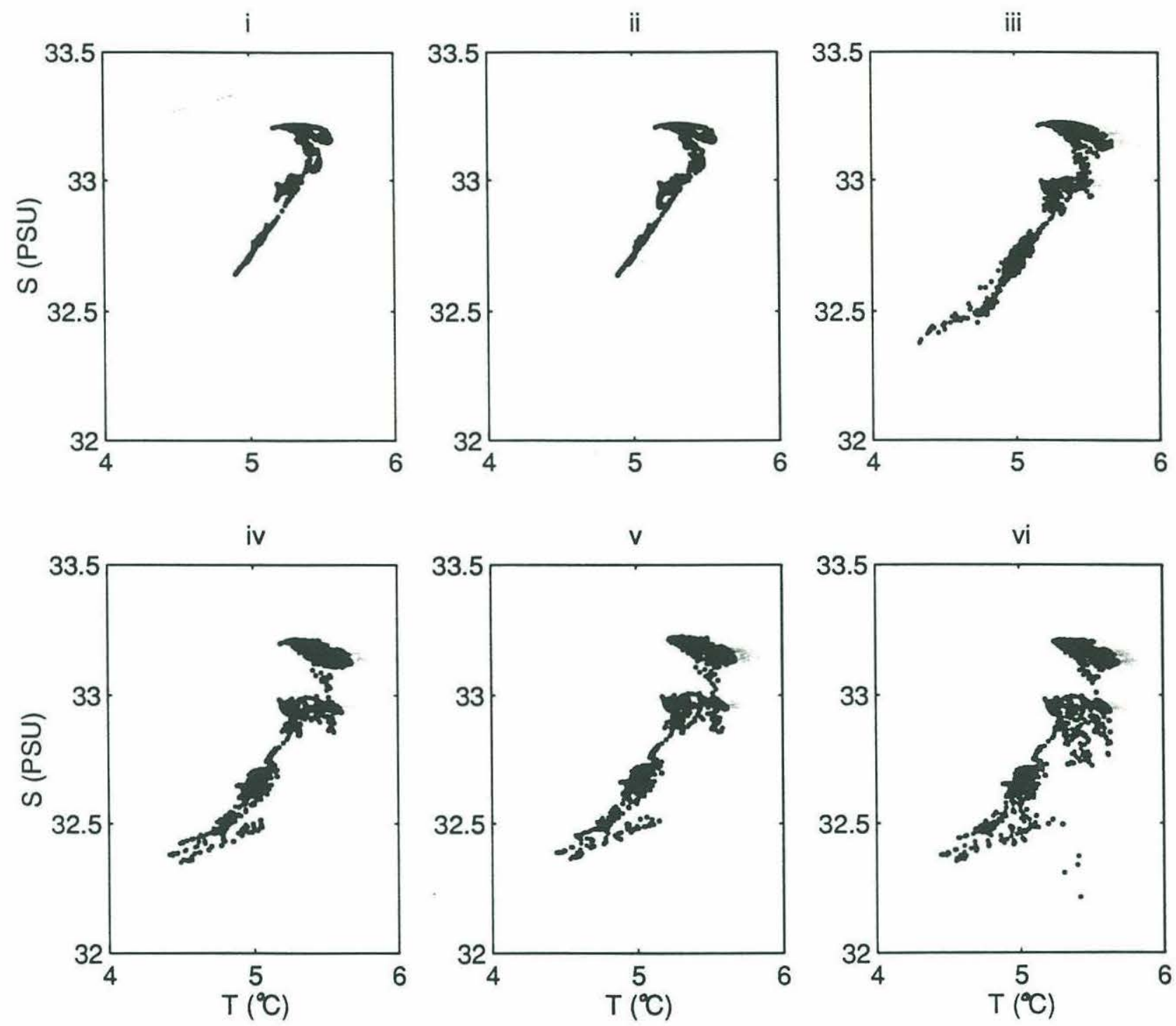

Figure 7: Temperature-salinity (T-S) characteristics from Seacat data taken at $10.90 \mathrm{~m}(\mathrm{i})$, $29.40 \mathrm{~m}$ (ii), $49.90 \mathrm{~m}$ (iii), $64.95 \mathrm{~m}$ (iv), $69.75 \mathrm{~m}$ (v), and $74.50 \mathrm{~m}$ (vi) above the bottom. 

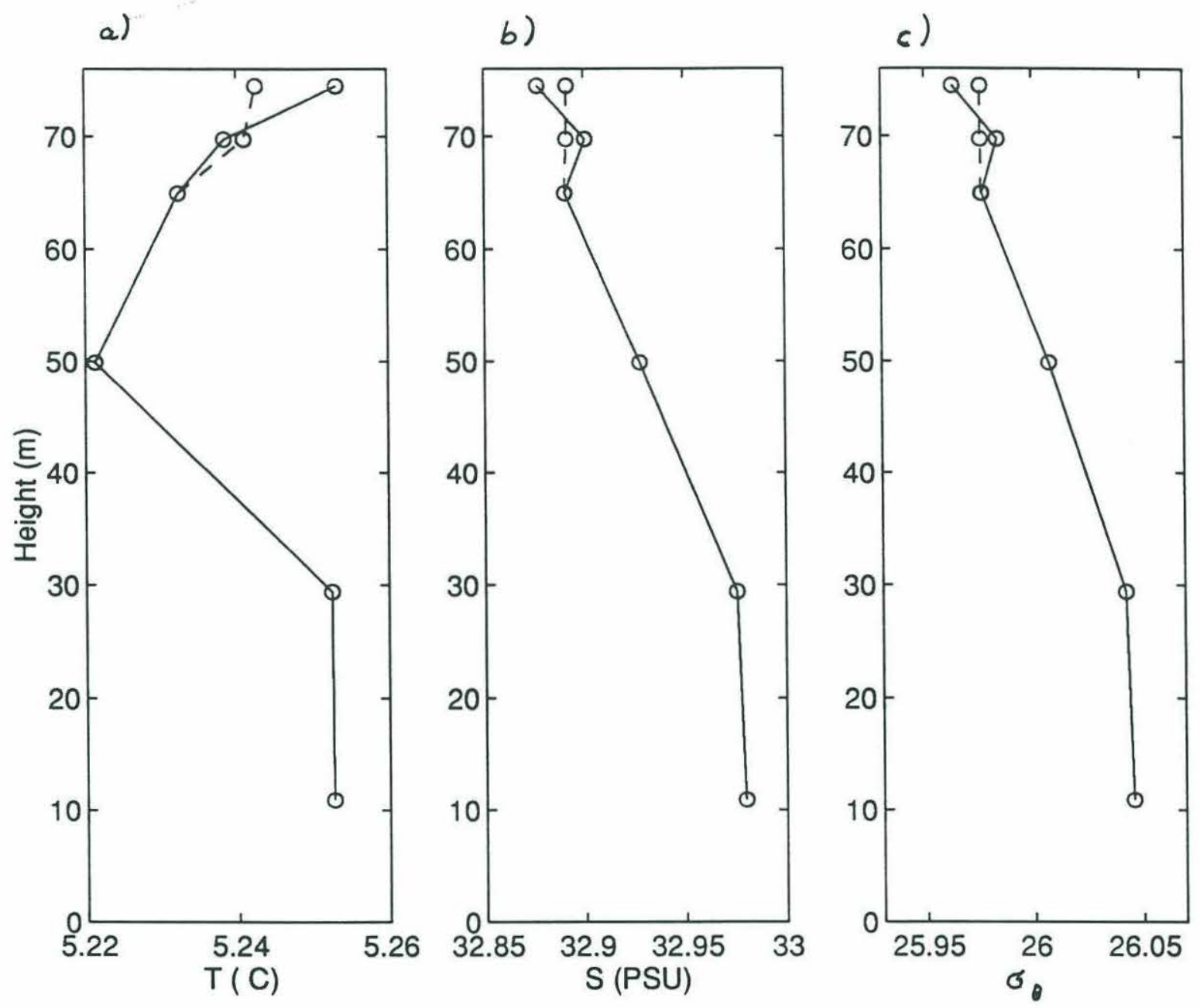

Figure 8: Profiles of mean temperature T (a), salinity S (b), and potential density $\sigma_{\theta}(\mathrm{c})$ from Seacat data for the time period from yearday 50 to 80 . Dashed lines represent mean values obtained after isolated events of high stratification near the surface have been excluded. 

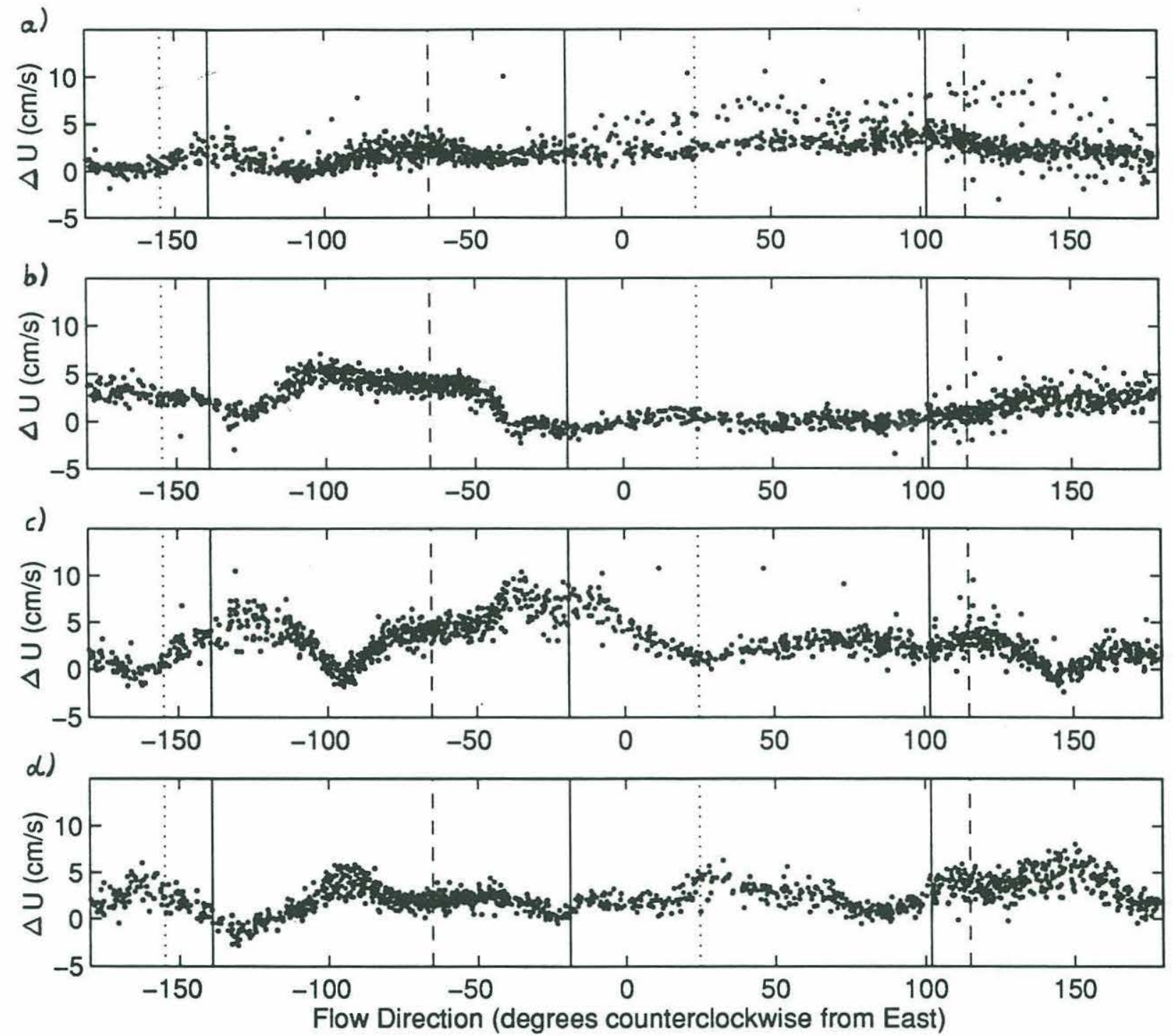

Figure 9: Differences in measured speeds $\Delta U$ between pods 5 and 4 (a), 4 and 3 (b), 3 and 2 (c), and 2 and 1 (d) as a function of mean flow direction at pods 1-5. Flow orientations are eastward at $0^{\circ}$, and northward at $90^{\circ}$. Solid lines represent directions where flow obstruction due to the tripod legs is possible. Dashed and dotted lines mark the orientation of the major and minor axes of the $\mathrm{M}_{2}$ current ellipse, respectively. 

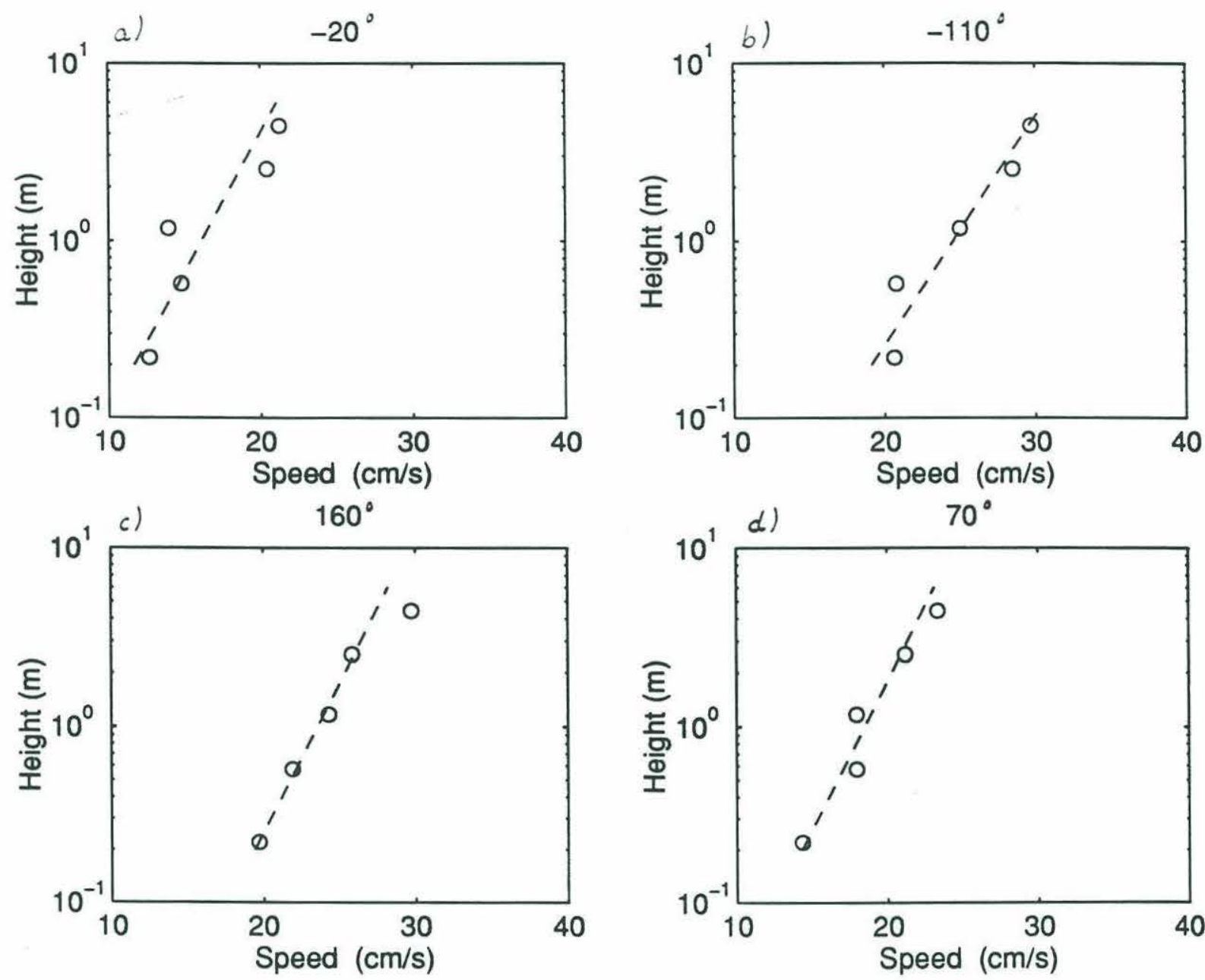

Figure 10: Averaged BASS velocity profiles taken between yearday 50-80 within $\pm 5^{\circ}$ from angles of largest increase and decrease of tidal current speeds, $U$. Profiles are displayed on a semi-logarithmic (base 10) scale. Dashed lines represent results from a least-squares logarithmic fit to measured current speeds at pods $1-4$. $\frac{\partial U}{\partial t}$ is largest around $-20^{\circ}(\mathrm{a})$ and $160^{\circ}$ (c), and smallest around $-110^{\circ}(\mathrm{b})$ and $70^{\circ}(\mathrm{d})$, where minus signs denote clockwise from east. 

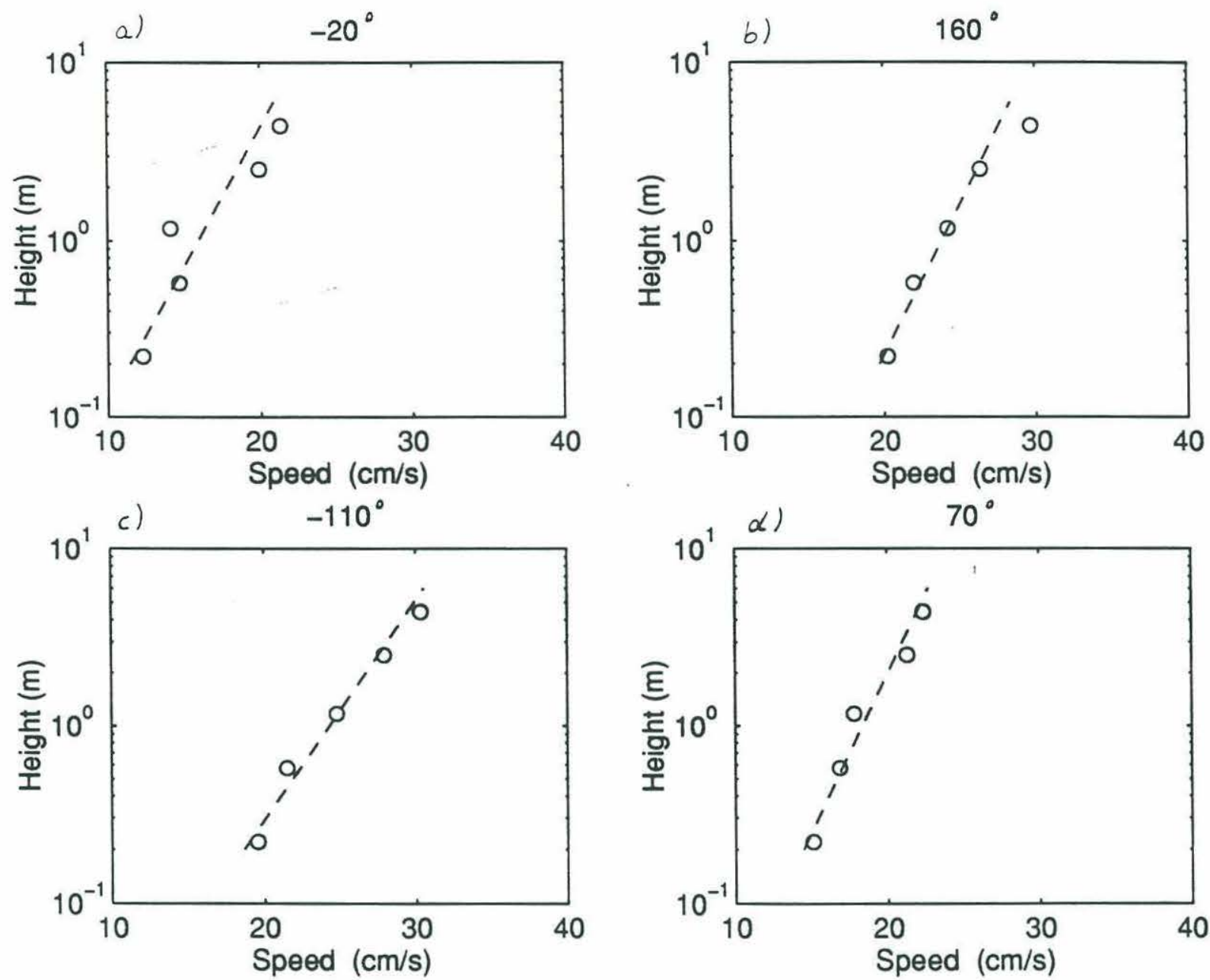

Figure 11: Averaged BASS velocity profiles taken between yearday 50-80 within $\pm 5^{\circ}$ from angles of largest increase and decrease of tidal current speeds, $U$, with their offset residuals being removed. Profiles are displayed on. a semi-logarithmic (base 10) scale. Dashed lines represent results from a least-squares logarithmic fit to measured current speeds at pods 1-4. $\frac{\partial U}{\partial t}$ is largest around $-20^{\circ}$ (a) and $160^{\circ}$ (c), and smallest around $-110^{\circ}$ (b) and $70^{\circ}$ (d), where minus signs denote clockwise from east. 


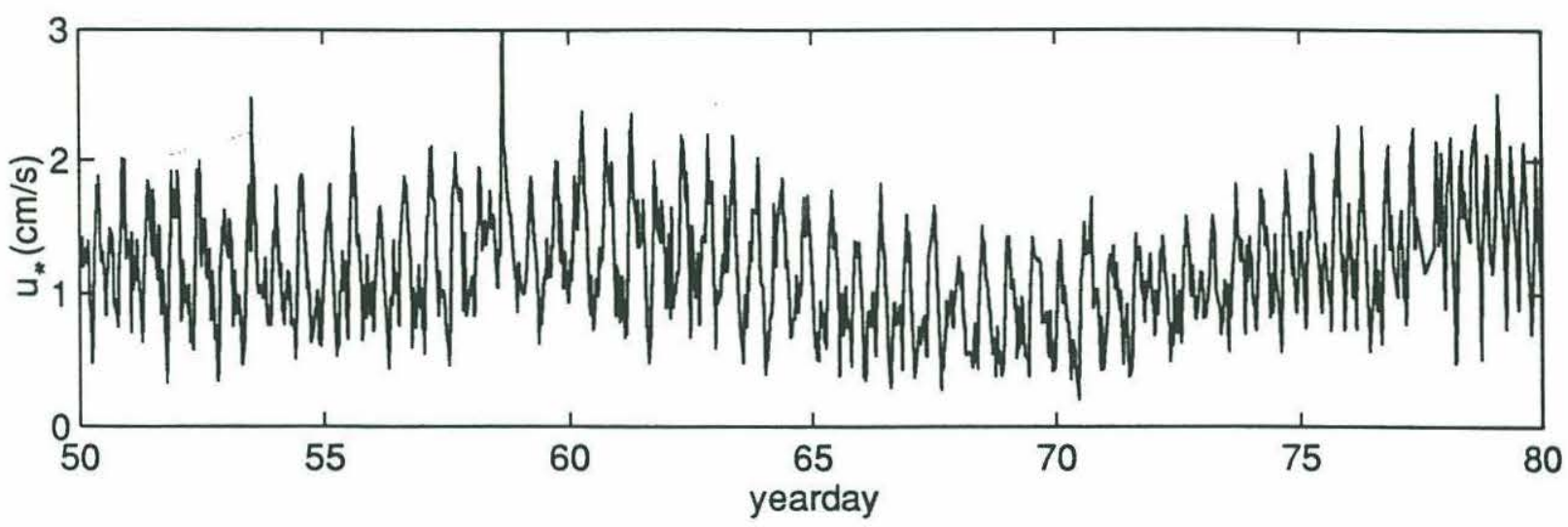

Figure 12: Time series of friction velocities $u_{*}$ obtained from best-fit logarithmic profiles to BASS velocity data.
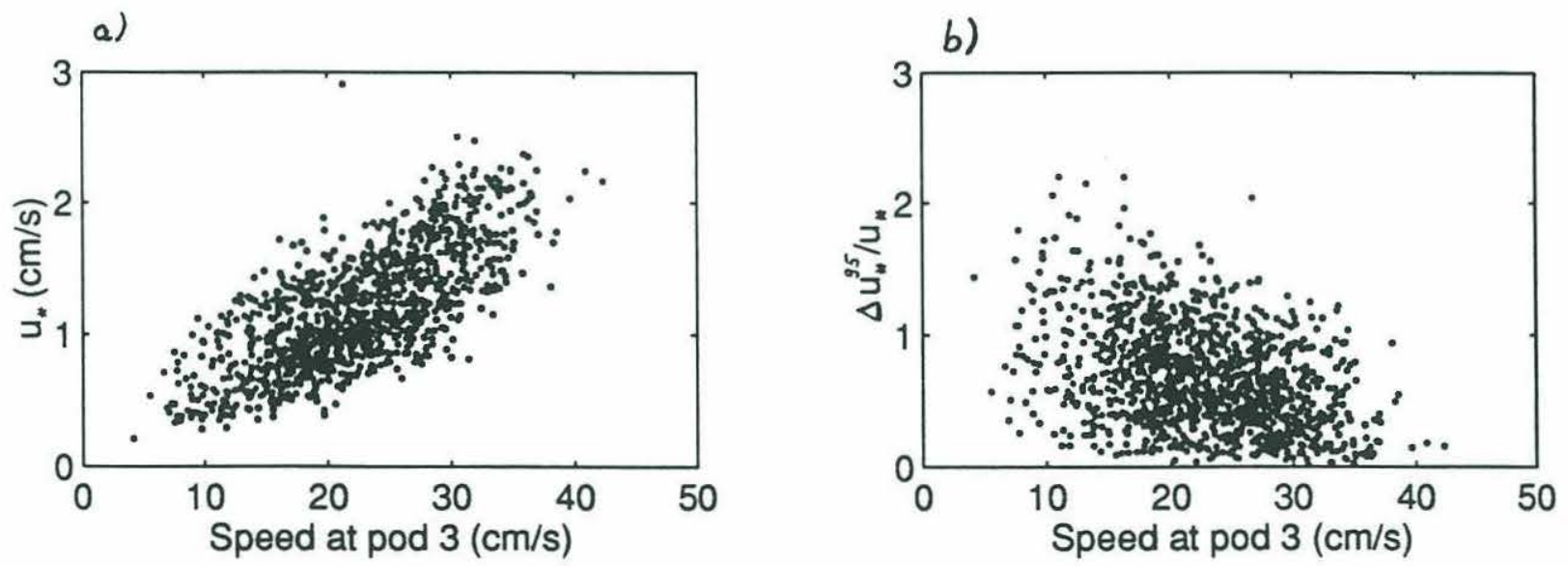

Figure 13: Estimated friction velocities $u_{*}(a)$, and ratios $\frac{\Delta u_{*}^{95}}{u_{*}}$ (b) versus speed at pod 3, where $\Delta u_{*}^{95}$ is the $95 \%$ confidence interval of $u_{*}$. 

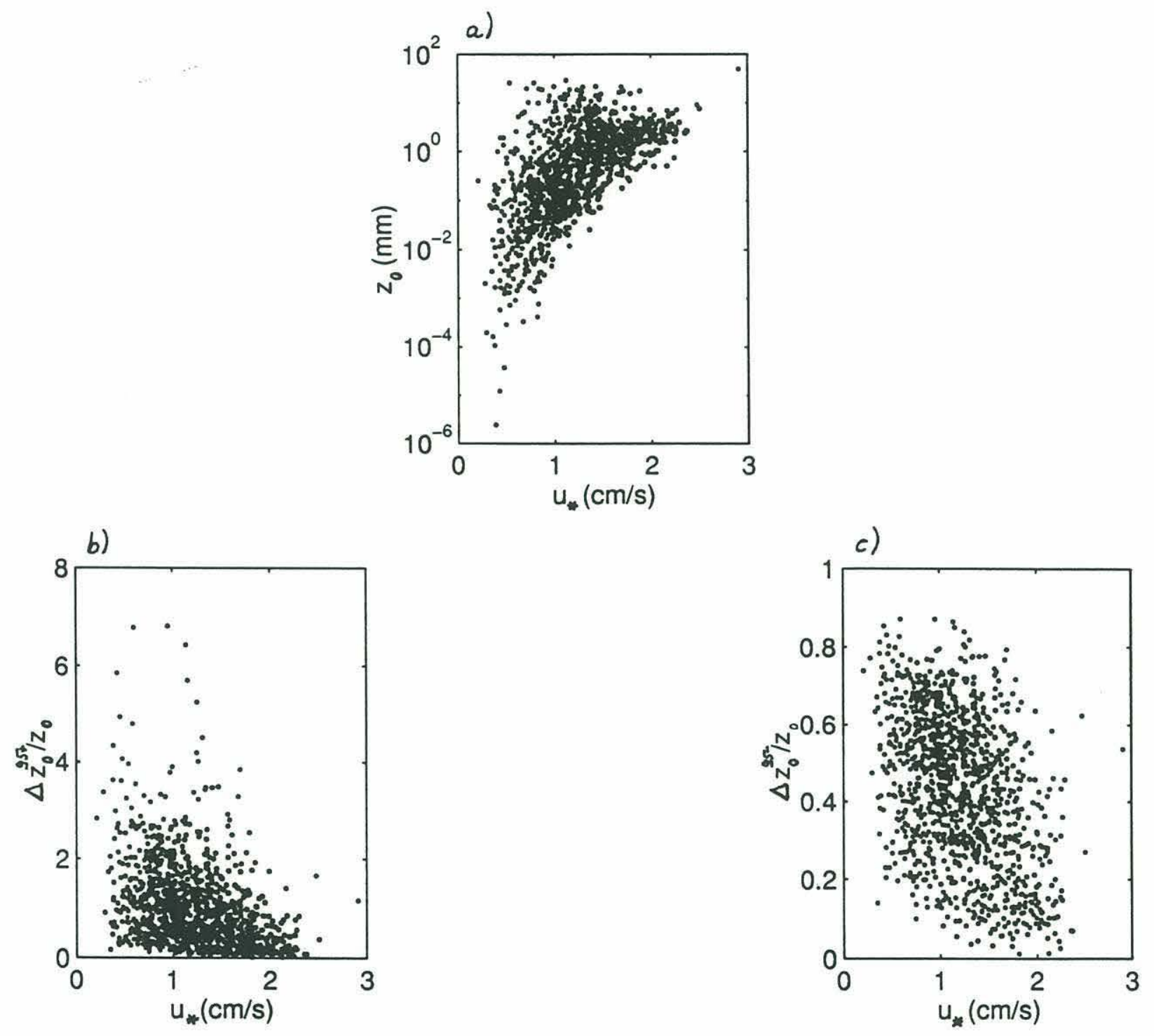

Figure 14: Estimated bottom roughness $z_{0}(\mathrm{a})$, ratios $\frac{\Delta z_{0}^{95+}}{z_{0}}(\mathrm{~b})$, and ratios $\frac{\Delta z_{0}^{95-}}{z_{0}}$ (c) versus $u_{*}$, where $\Delta z_{0}^{95+}$ and $\Delta z_{0}^{95-}$ are the upper and lower $95 \%$ confidence intervals of $z_{0}$, respectively. Bottom roughness estimates (a) are displayed on a semilogarithmic (base 10) scale. 


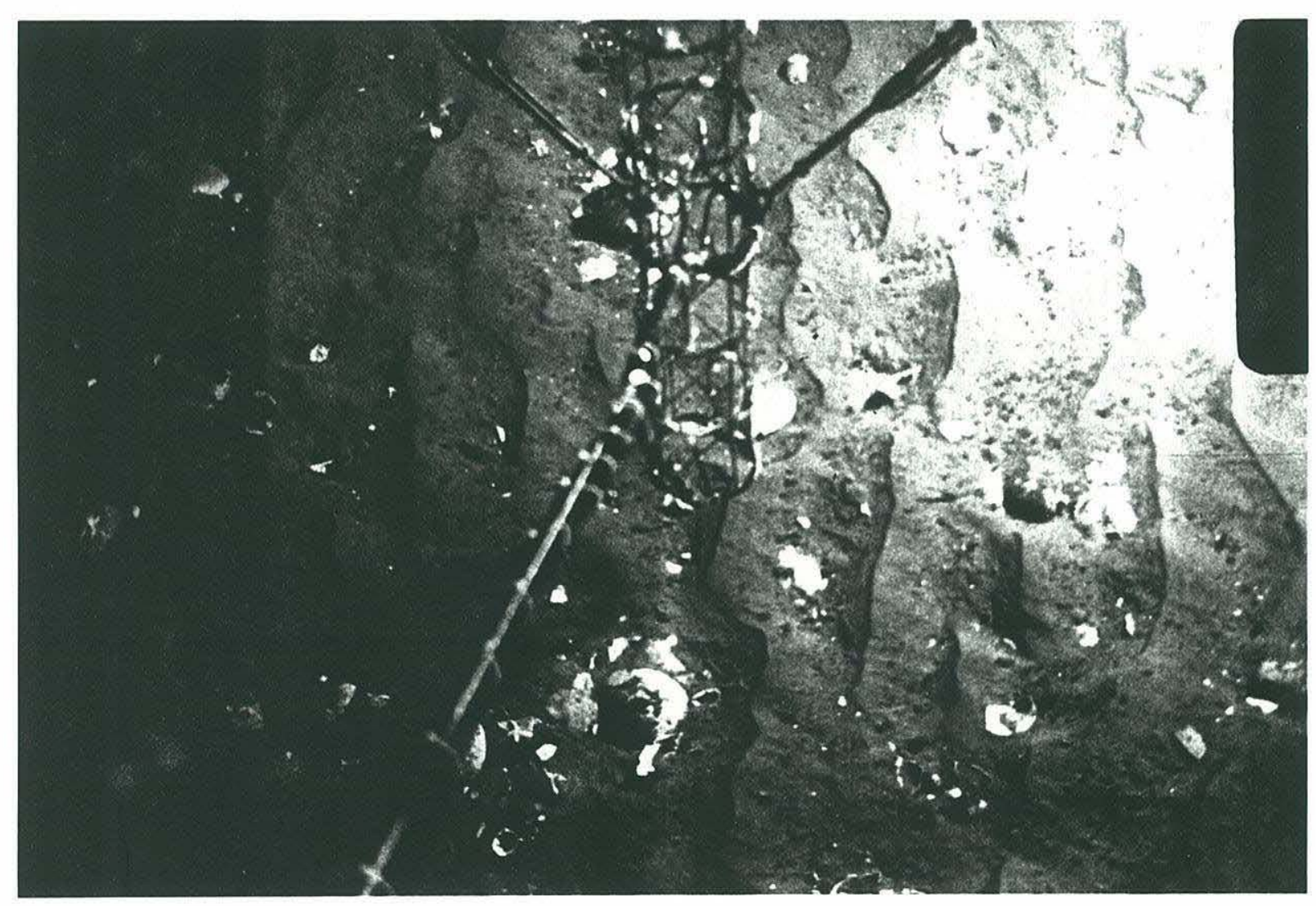

Figure 15: Picture of the sea floor displaying the presence of sand ripples. Cables in the lower left corner are connected to the camera and are $\approx 40^{\circ}$ counterclockwise from east. Sand ripples are aligned north eastward. 

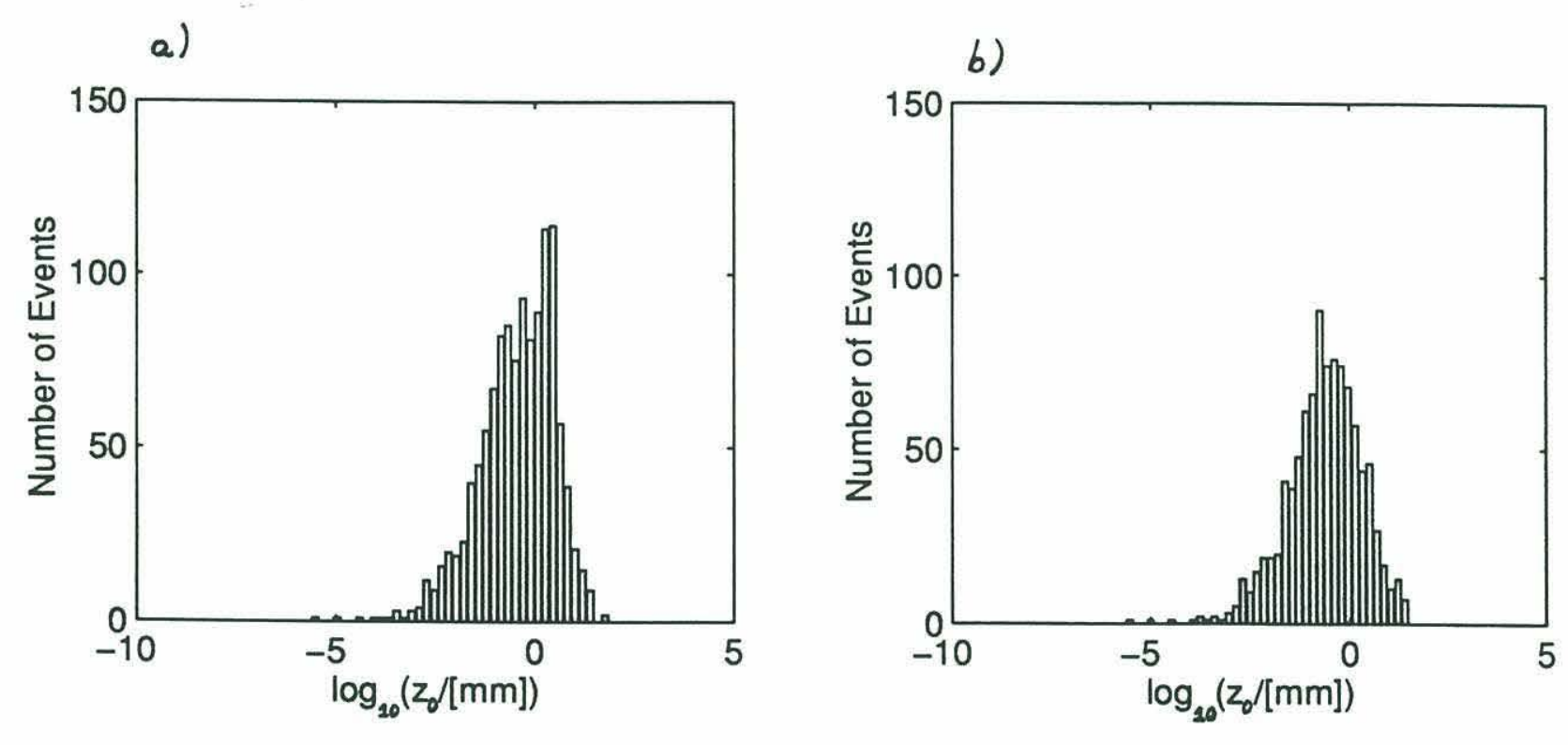

Figure 16: Histograms of bottom roughness $\log _{10}\left(z_{0}\right)$, where $z_{0}$ is in millimeters. All flow directions are included in (a), while directions believed to be affected by the battery case $\left(35^{\circ}-75^{\circ}\right.$ clockwise from east) are excluded in (b). 


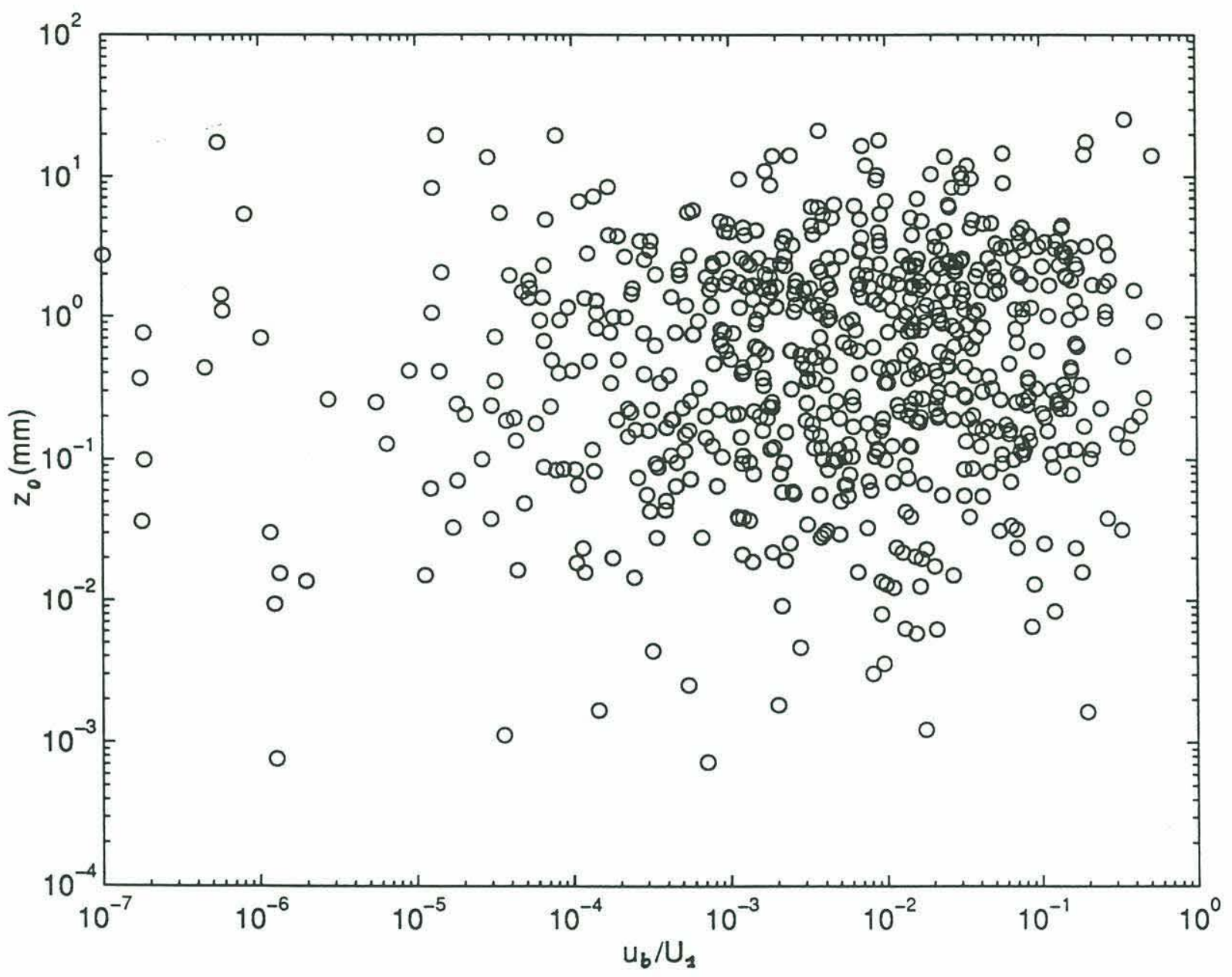

Figure 17: Estimates of bottom roughness $z_{0}$ versus ratios $\frac{u_{b}}{U_{1}}$ on a logarithmic (base 10) scale. $U_{1}$ is the measured current speed at pod 1 , and $u_{b}$ is the orbital wave velocity near the bottom. 

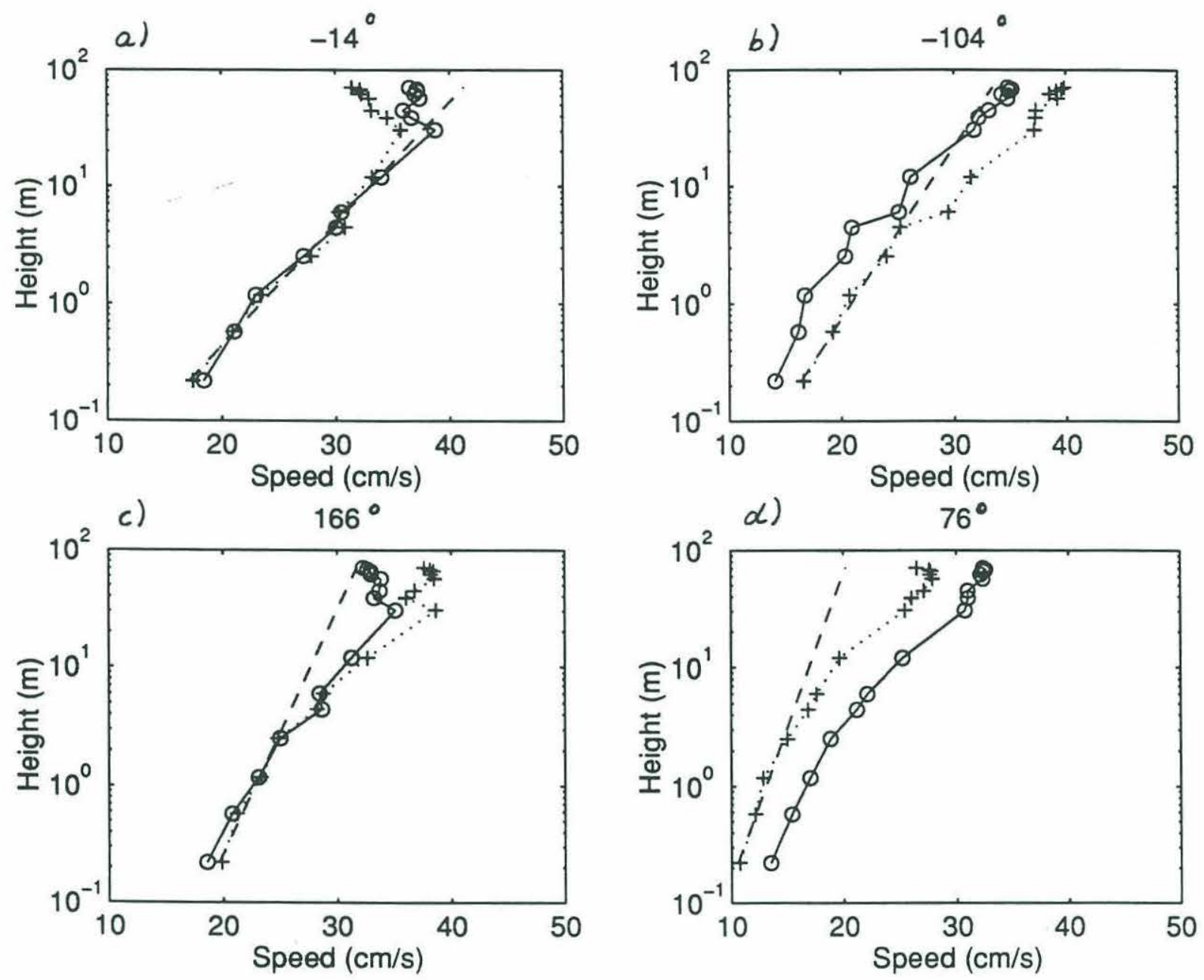

Figure 18: Vertical structure of the $\mathrm{M}_{2}$ tidal velocities (solid lines) and total current speeds (dotted lines) from BASS and VMCM data taken between yearday 50-80. Profiles are displayed on a semilogarithmic (base 10) scale. Dashed lines represent results from a least-squares logarithmic fit to measured current speeds at pods 1-4. Profiles are from averaged data, where the average was taken of current measurements with flow directions less than $\pm 5^{\circ}$ from angles of maximum acceleration and deceleration of the near surface tidal speeds $U . \frac{\partial U}{\partial t}$ is largest around $-14^{\circ}(\mathrm{a})$ and $166^{\circ}(\mathrm{c})$, and smallest around $-104^{\circ}$ (b) and $76^{\circ}(\mathrm{d})$, where minus signs denote clockwise from east. 

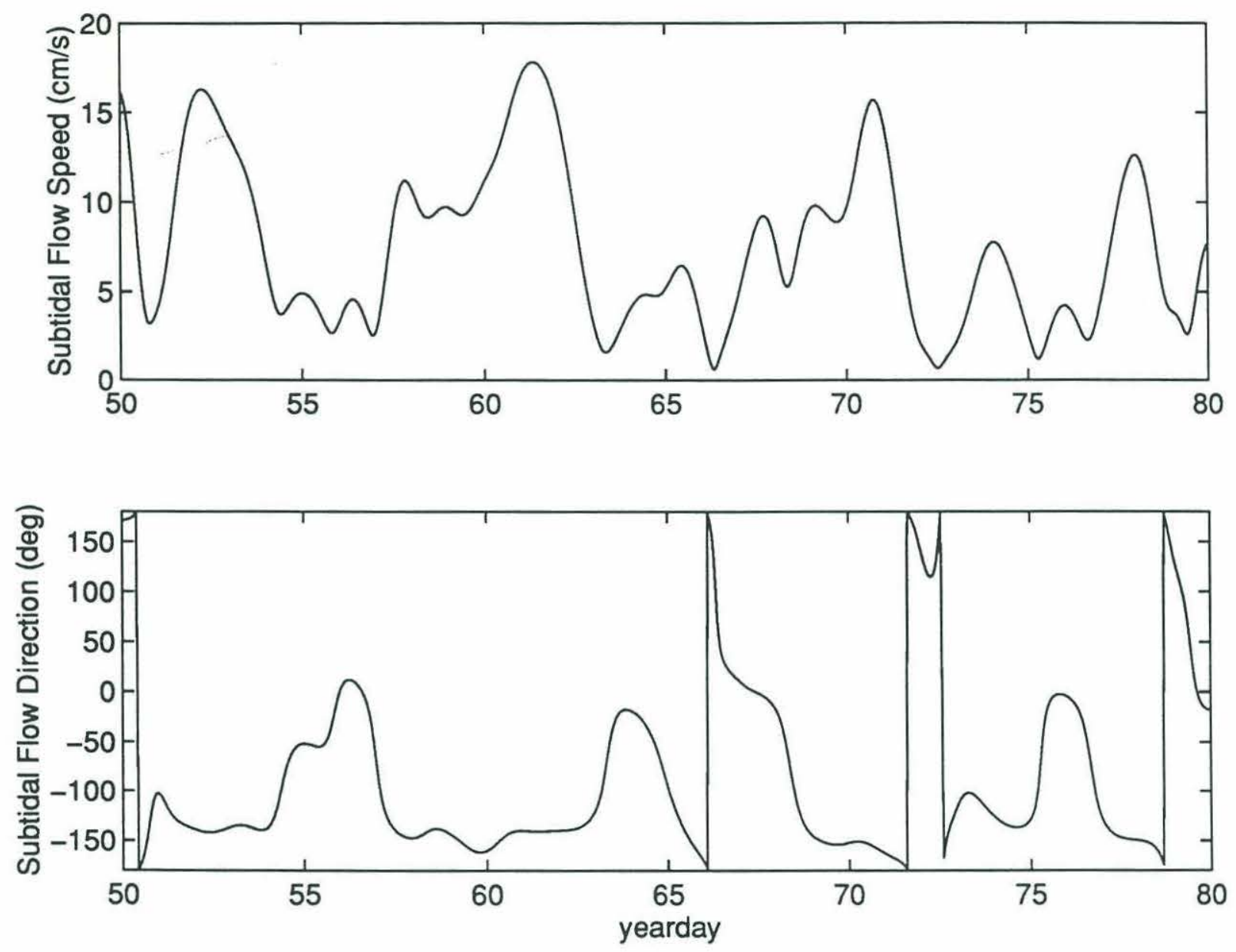

Figure 19: Time series of depth-averaged, low-pass filtered flow speeds (upper) and flow directions (lower). The low-pass filter applies to frequencies smaller than diurnal. Flow orientations are eastward at $0^{\circ}$, and northward at $90^{\circ}$. 
a)

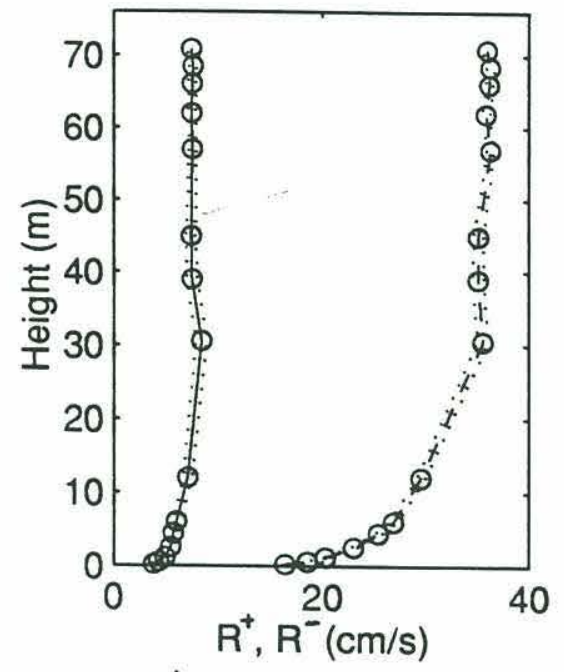

d)

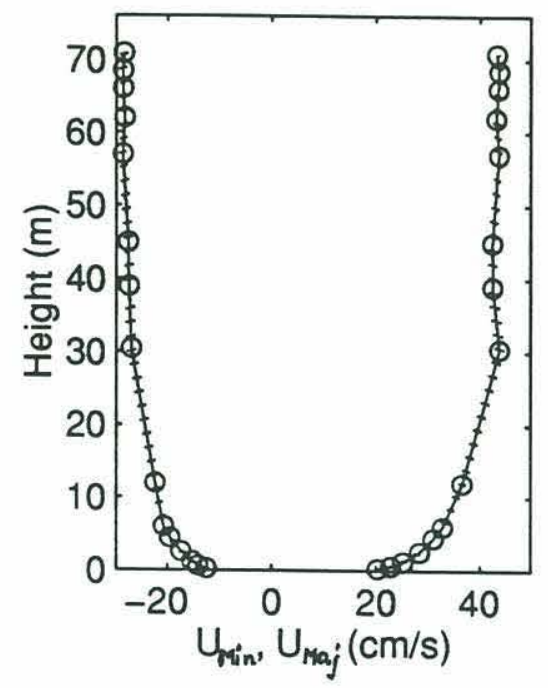

b)

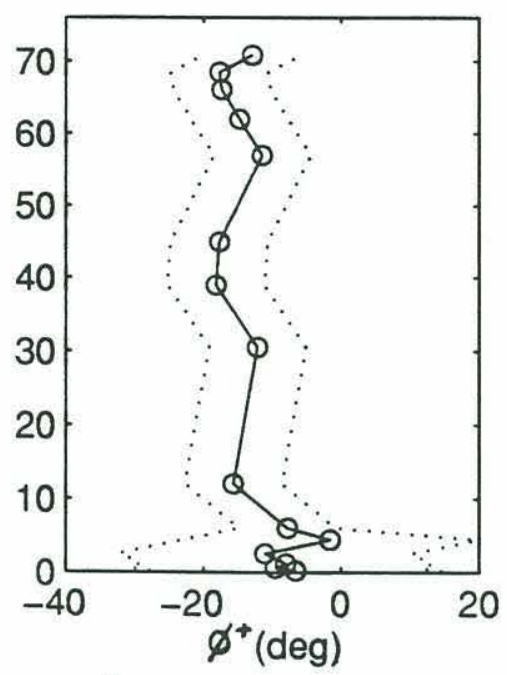

e)

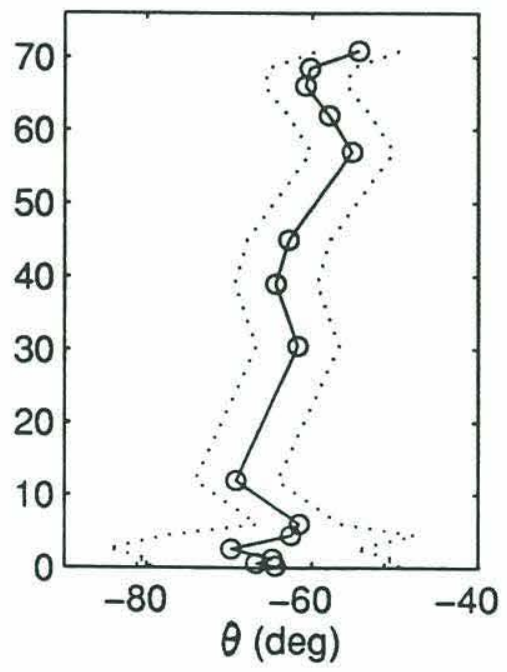

c)

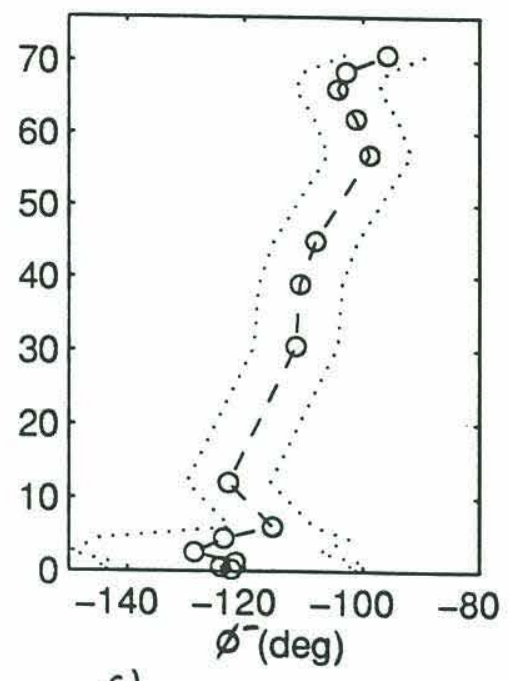

f)

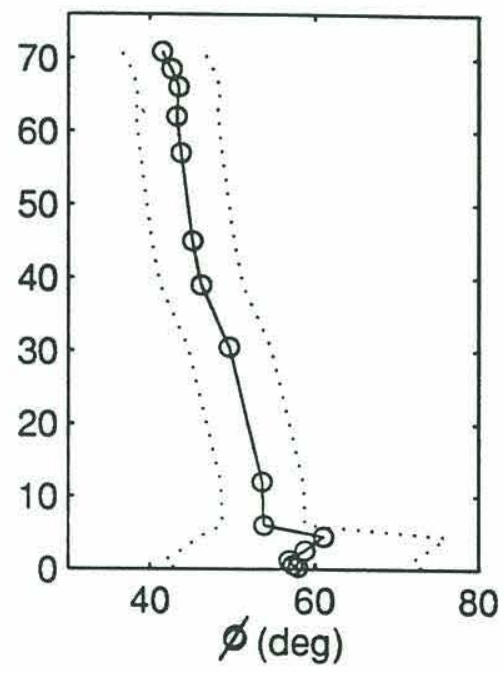

Figure 20: Vertical structure of the $M_{2}$ current ellipse obtained from tidal analysis of BASS and VMCM data for yearday 50-80. Profiles show the magnitudes of the rotary components $R^{+}$(solid line) and $R^{-}$(dashed line) (a), corresponding phase angles $\phi^{+}(\mathrm{b})$ and $\phi^{-}(\mathrm{c})$, current amplitudes $U_{M a j}$ and $U_{M i n}(\mathrm{~d})$, inclination of the major axis $\theta(\mathrm{e})$, and phase of the velocity vector $\phi(\mathrm{f})$. Dotted lines represent error bars. Inclination and phase angles of the rotary components are given with respect to east, where negative values are clockwise from east. Phase angles describe the orientation of the $M_{2}$ velocity vector at the beginning of the time series. 


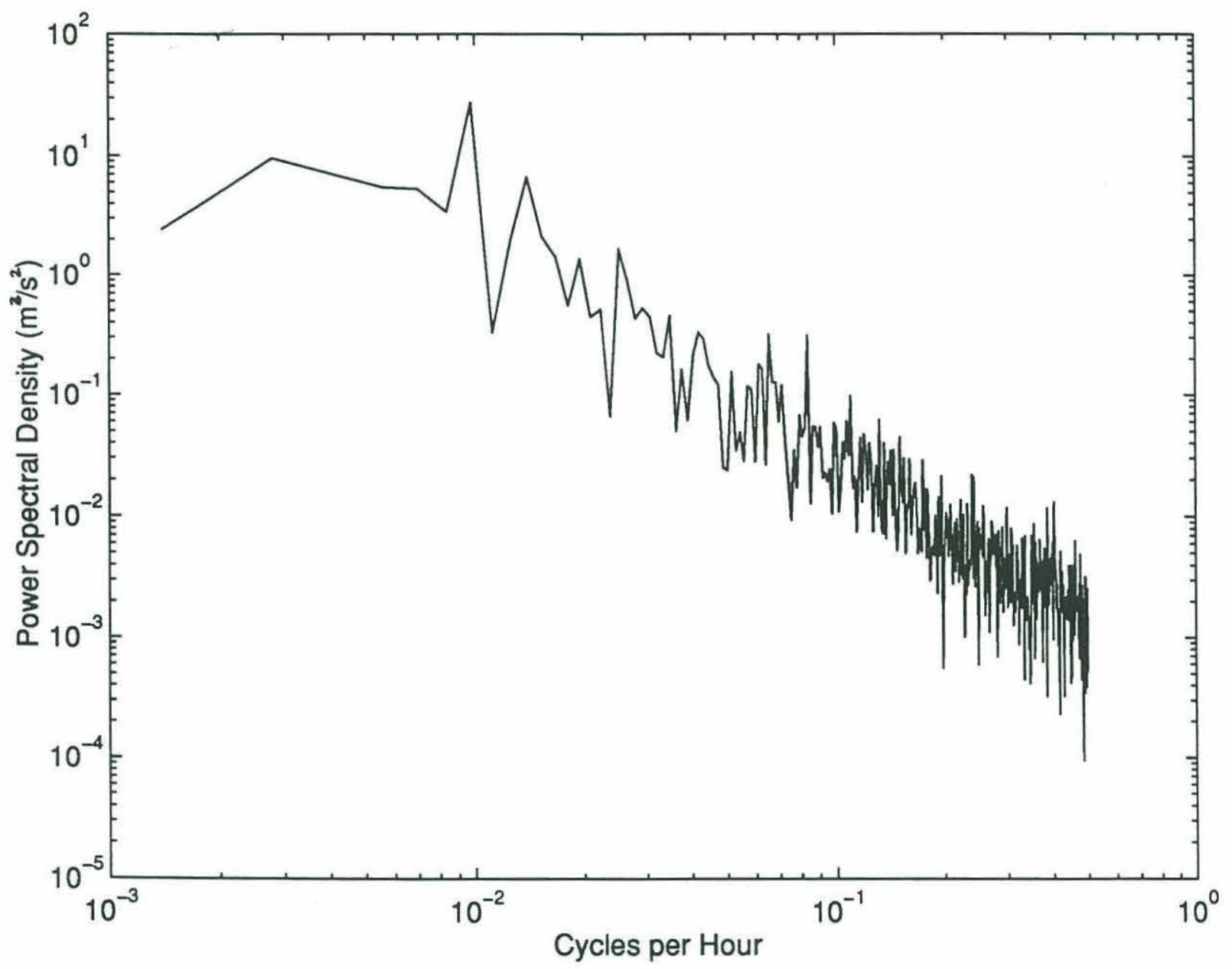

Figure 21: Kinetic energy spectrum of wind speeds measured by the IMET between yearday $50-80$. 
a)

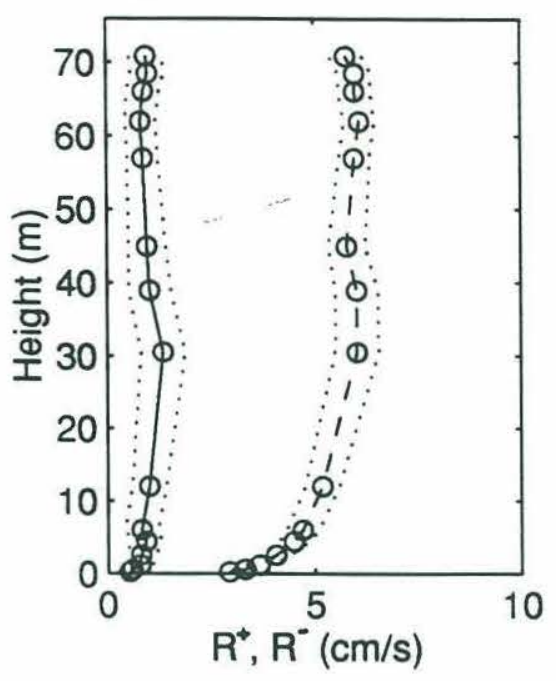

d)

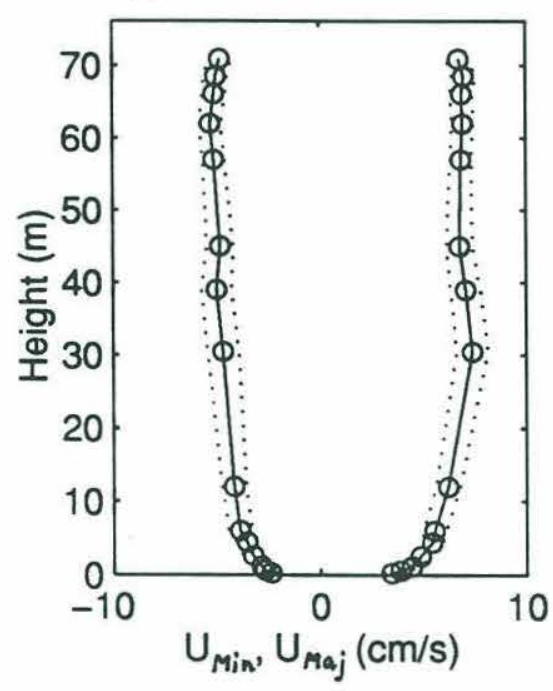

b)

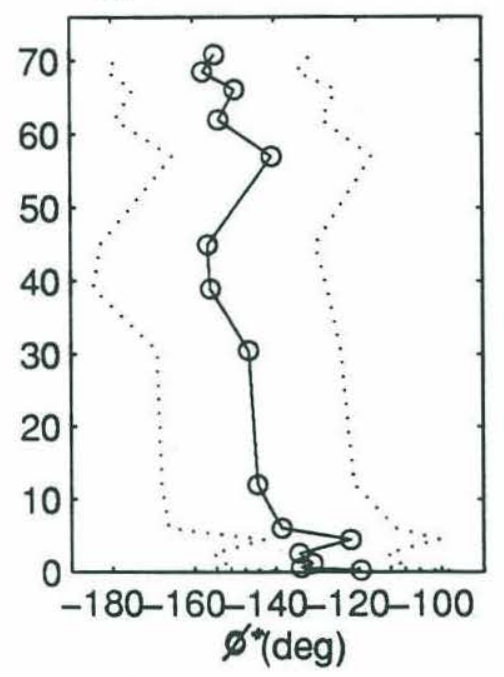

e)

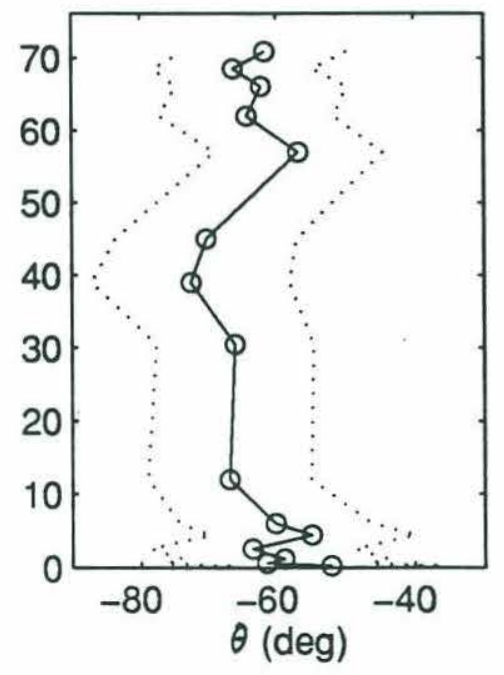

c)

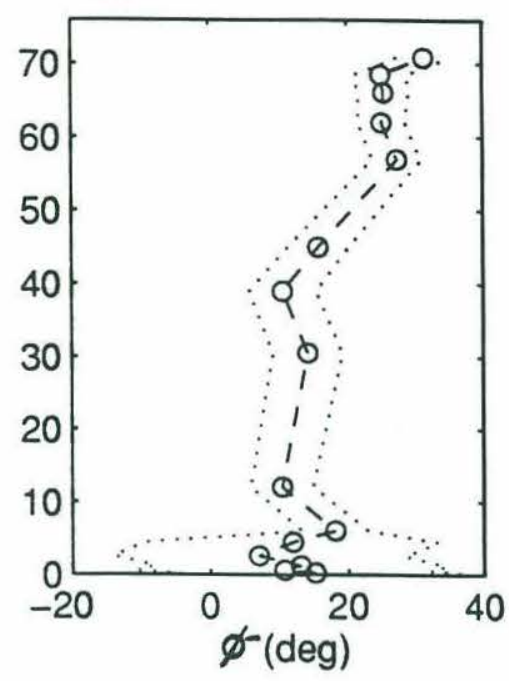

f)

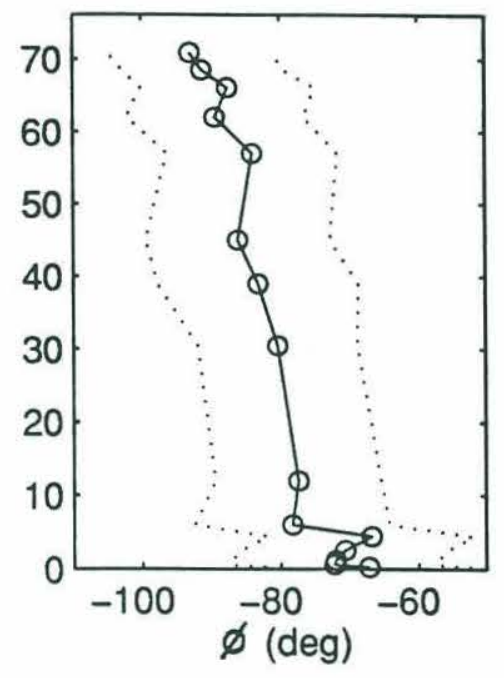

Figure 22: Vertical structure of the $\mathrm{N}_{2}$ current ellipse obtained from tidal analysis of BASS and VMCM data for yearday 50-80. Profiles show the magnitudes of the rotary components $R^{+}$(solid line) and $R^{-}$(dashed line) (a), corresponding phase angles $\phi^{+}(\mathrm{b})$ and $\phi^{-}(\mathrm{c})$, current amplitudes $U_{M a j}$ and $U_{M i n}(\mathrm{~d})$, inclination of the major axis $\theta$ (e), and phase of the velocity vector $\phi(f)$. Dotted lines represent error bars. Inclination and phase angles of the rotary components are given with respect to east, where negative values are clockwise from east. Phase angles describe the orientation of the $\mathrm{N}_{2}$ velocity vector at the beginning of the time series. 

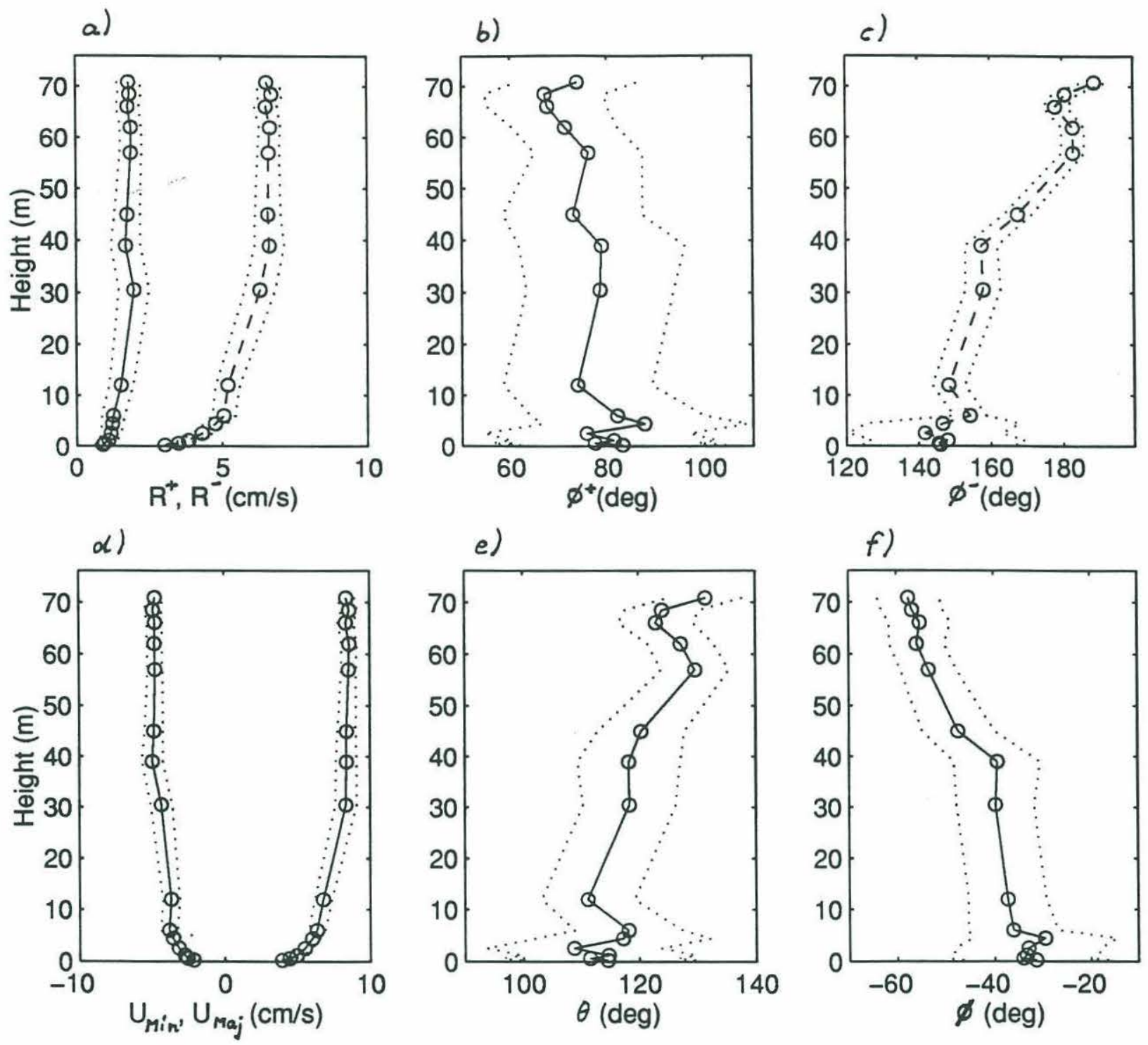

e)
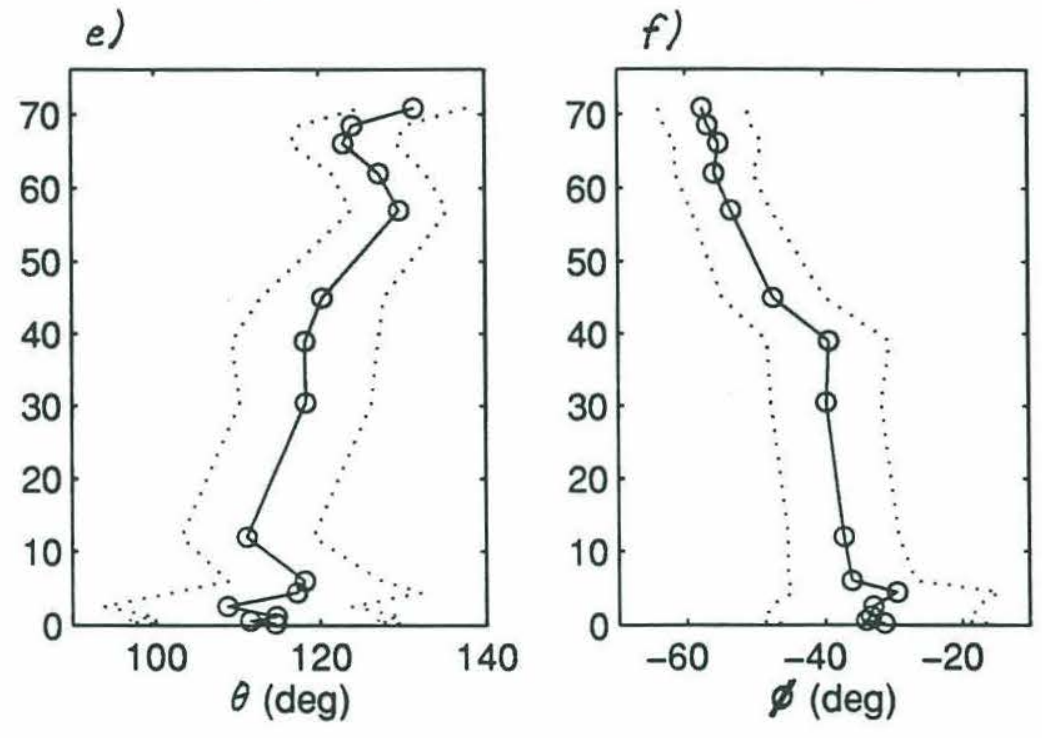

Figure 23: Vertical structure of the $S_{2}$ current ellipse obtained from tidal analysis of BASS and VMCM data for yearday 50-80. Profiles show the magnitudes of the rotary components $R^{+}$(solid line) and $R^{-}$(dashed line) (a), corresponding phase angles $\phi^{+}(\mathrm{b})$ and $\phi^{-}(\mathrm{c})$, current amplitudes $U_{M a j}$ and $U_{M i n}(\mathrm{~d})$, inclination of the major axis $\theta$ (e), and phase of the velocity vector $\phi(f)$. Dotted lines represent error bars. Inclination and phase angles of the rotary components are given with respect to east, where negative values are clockwise from east. Phase angles describe the orientation of the $S_{2}$ velocity vector at the beginning of the time series. 
a)
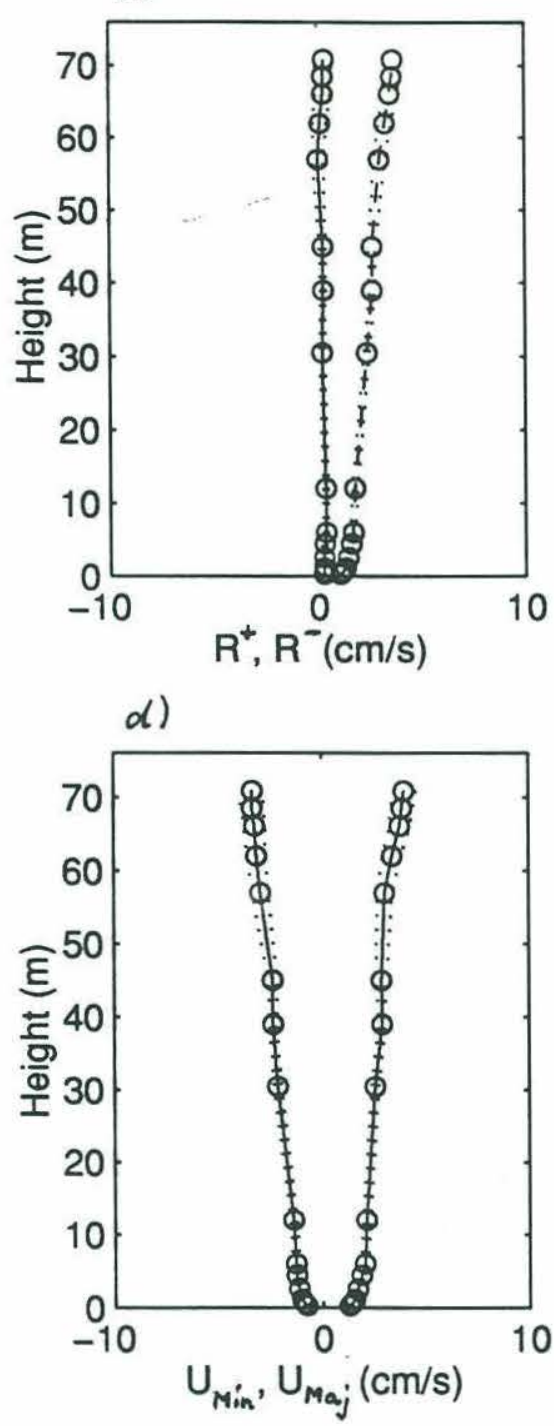

b)
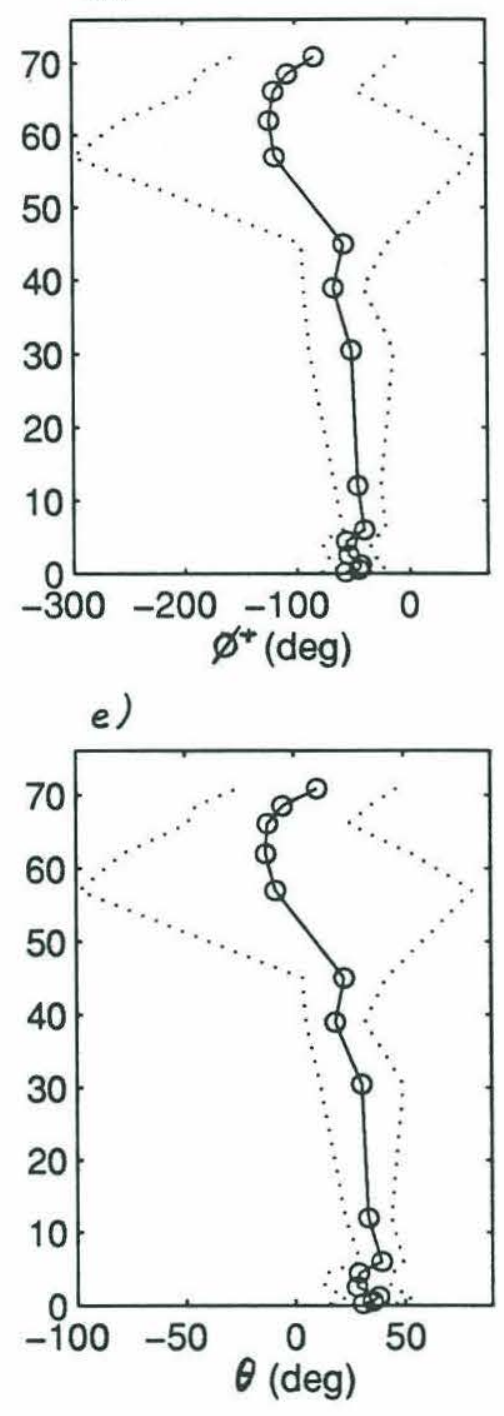
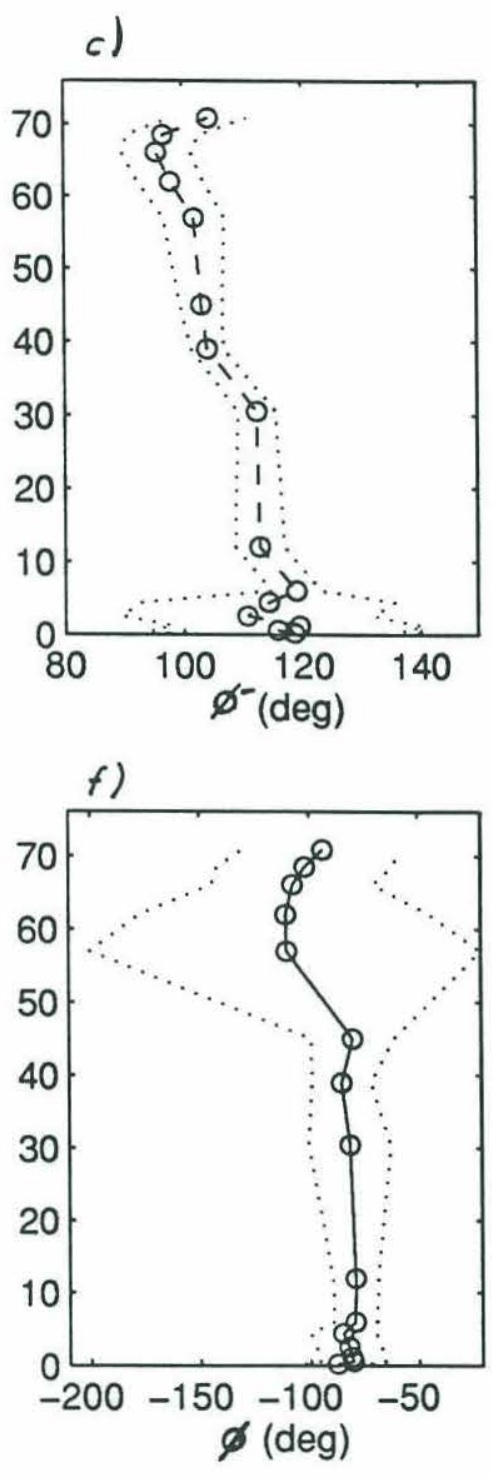

Figure 24: Vertical structure of the $K_{1}$ current ellipse obtained from tidal analysis of BASS and VMCM data for yearday 50-80. Profiles show the magnitudes of the rotary components $R^{+}$(solid line) and $R^{-}$(dashed line) (a), corresponding phase angles $\phi^{+}(\mathrm{b})$ and $\phi^{-}(\mathrm{c})$, current amplitudes $U_{M a j}$ and $U_{M i n}(\mathrm{~d})$, inclination of the major axis $\theta$ (e), and phase of the velocity vector $\phi(\mathrm{f})$. Dotted lines represent error bars. Inclination and phase angles of the rotary components are given with respect to east, where negative values are clockwise from east. Phase angles describe the orientation of the $K_{1}$ velocity vector at the beginning of the time series. 
a)

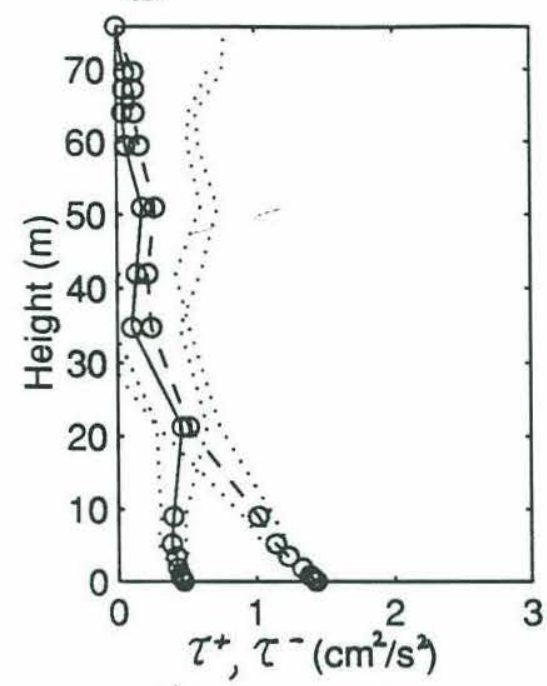

d)

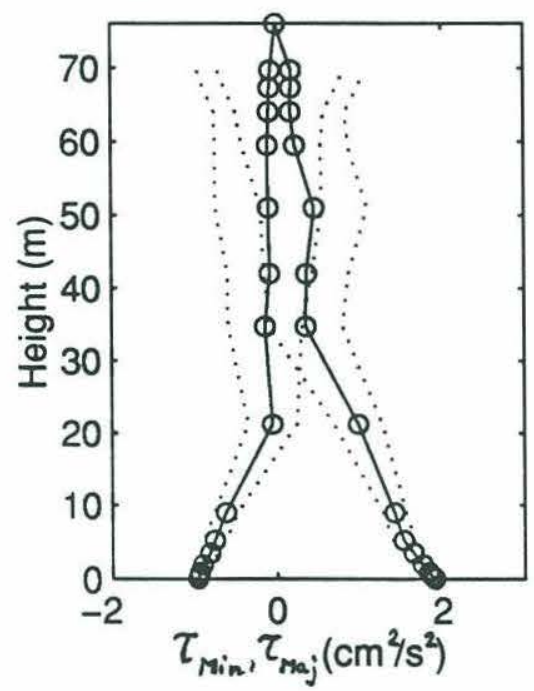

b)

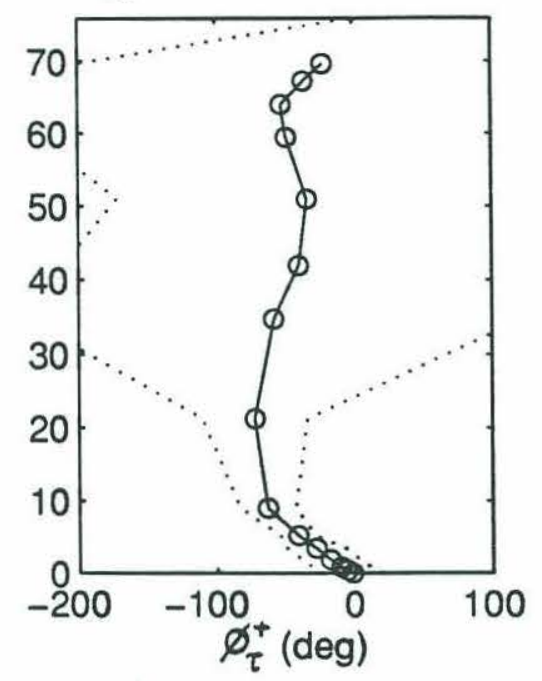

e)

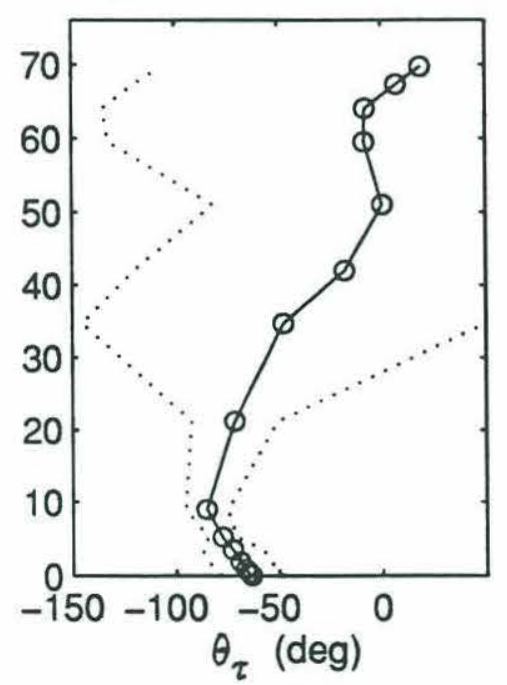

c)

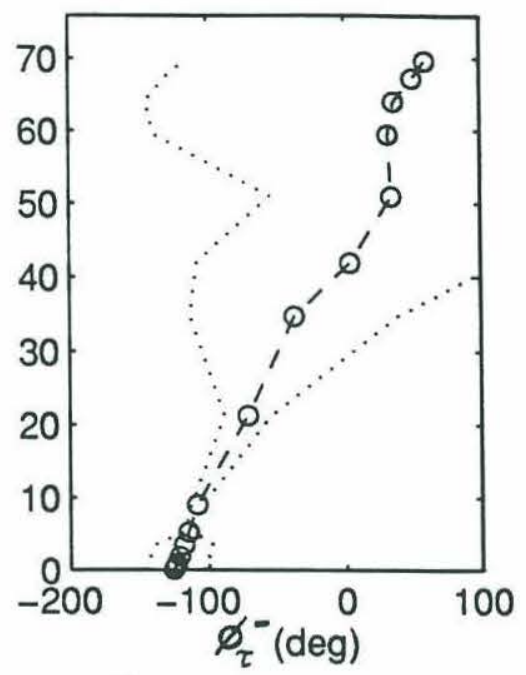

f)

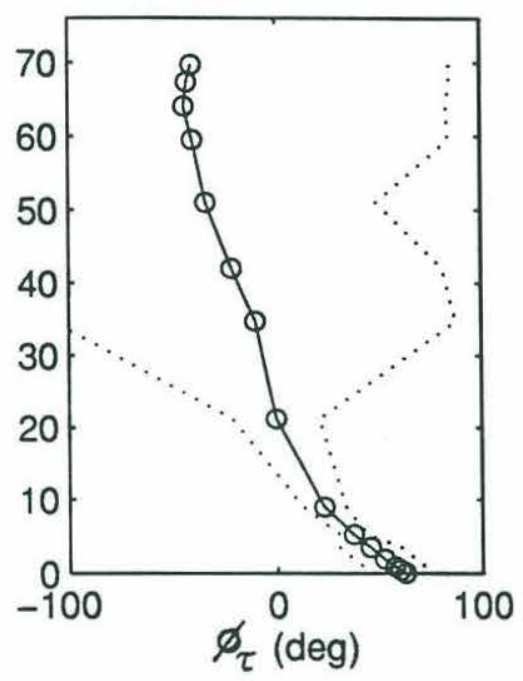

Figure 25: Profiles of the $M_{2}$ stress ellipse obtained from vertical integration of the momentum equations, using the BASS bottom stress estimates as a lower boundary condition. Profiles show the magnitudes of the rotary components $\tau^{+}$(solid line) and $\tau^{-}$(dashed line) (a), phase angles $\phi_{\tau}^{+}$(b) and $\phi_{\tau}^{-}$(c), current amplitudes $\tau_{M a j}$ and $\tau_{M i n}$ (d), inclination of the major axis $\theta_{\tau}(\mathrm{e})$, and phase of the stress vector $\phi_{\tau}(\mathrm{f})$. Dotted lines represent error bars. Inclination and phase angles of the rotary components are given with respect to east, where negative values are clockwise from east. Phase angles describe the orientation of the $\mathrm{M}_{2}$ stress vector at the beginning of the time series. 
a)

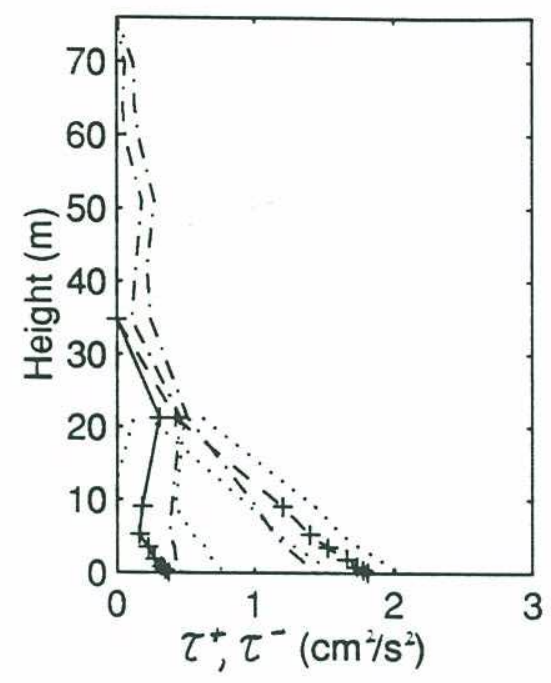

d)

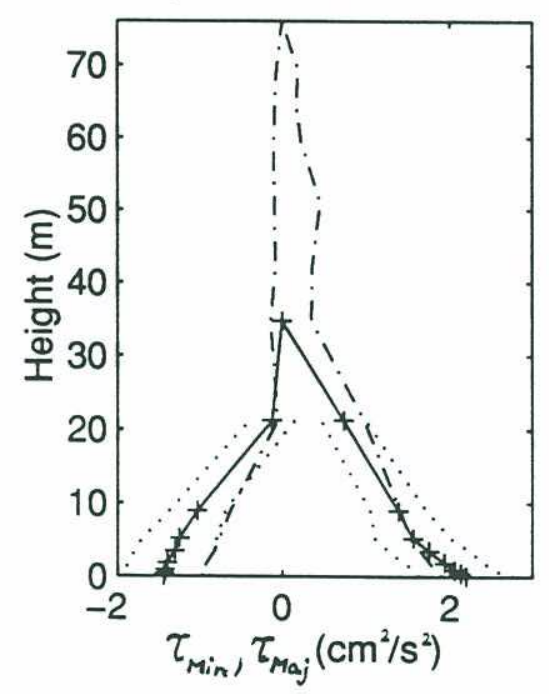

b)

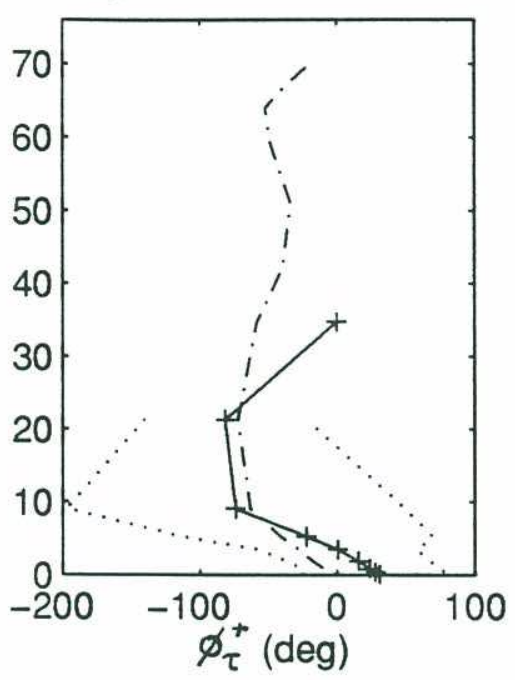

e)

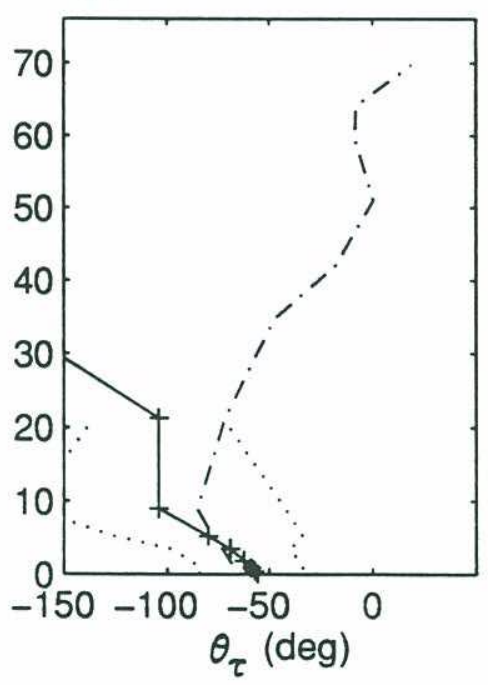

c)

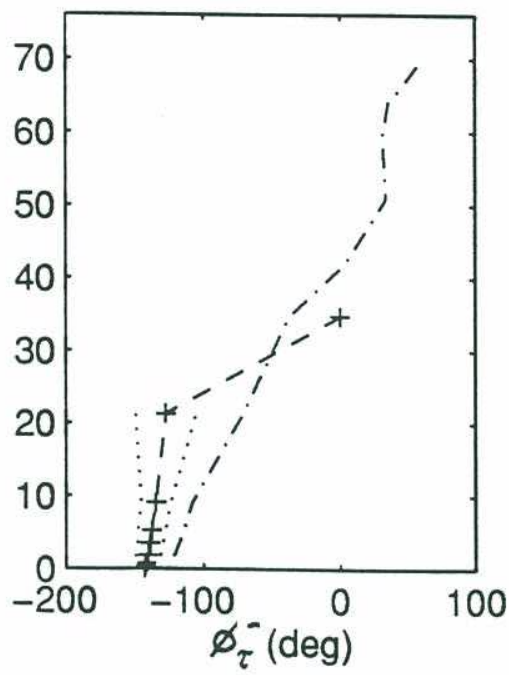

f)

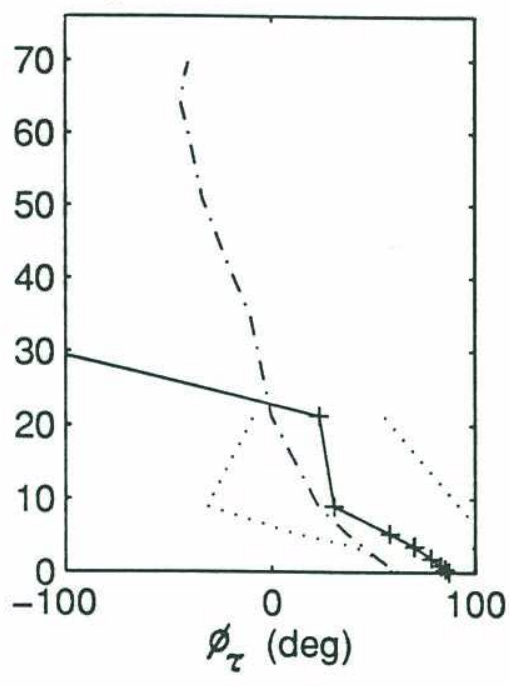

Figure 26: Profiles of the $\mathrm{M}_{2}$ stress ellipse parameters obtained from vertical integration of the velocity defect, assuming a region of zero stress divergence at heights $z>35 \mathrm{~m}$. Dashdotted lines correspond to results displayed in Figure 25. Profiles show the magnitudes of the rotary components $\tau^{+}$(solid line) and $\tau^{-}$(dashed line) (a), phase angles $\phi_{\tau}^{+}(\mathrm{b})$ and $\phi_{\tau}^{-}(\mathrm{c})$, current amplitudes $\tau_{M a j}$ and $\tau_{M i n}(\mathrm{~d})$, inclination of the major axis $\theta_{\tau}(\mathrm{e})$, and phase of the stress vector $\phi_{\tau}(\mathrm{f})$. Dotted lines represent error bars associated with the velocity defect method. Inclination and phase angles of the rotary components are given with respect to east, where negative values are clockwise from east. Phase angles describe the orientation of the $\mathrm{M}_{2}$ stress vector at the beginning of the time series. 

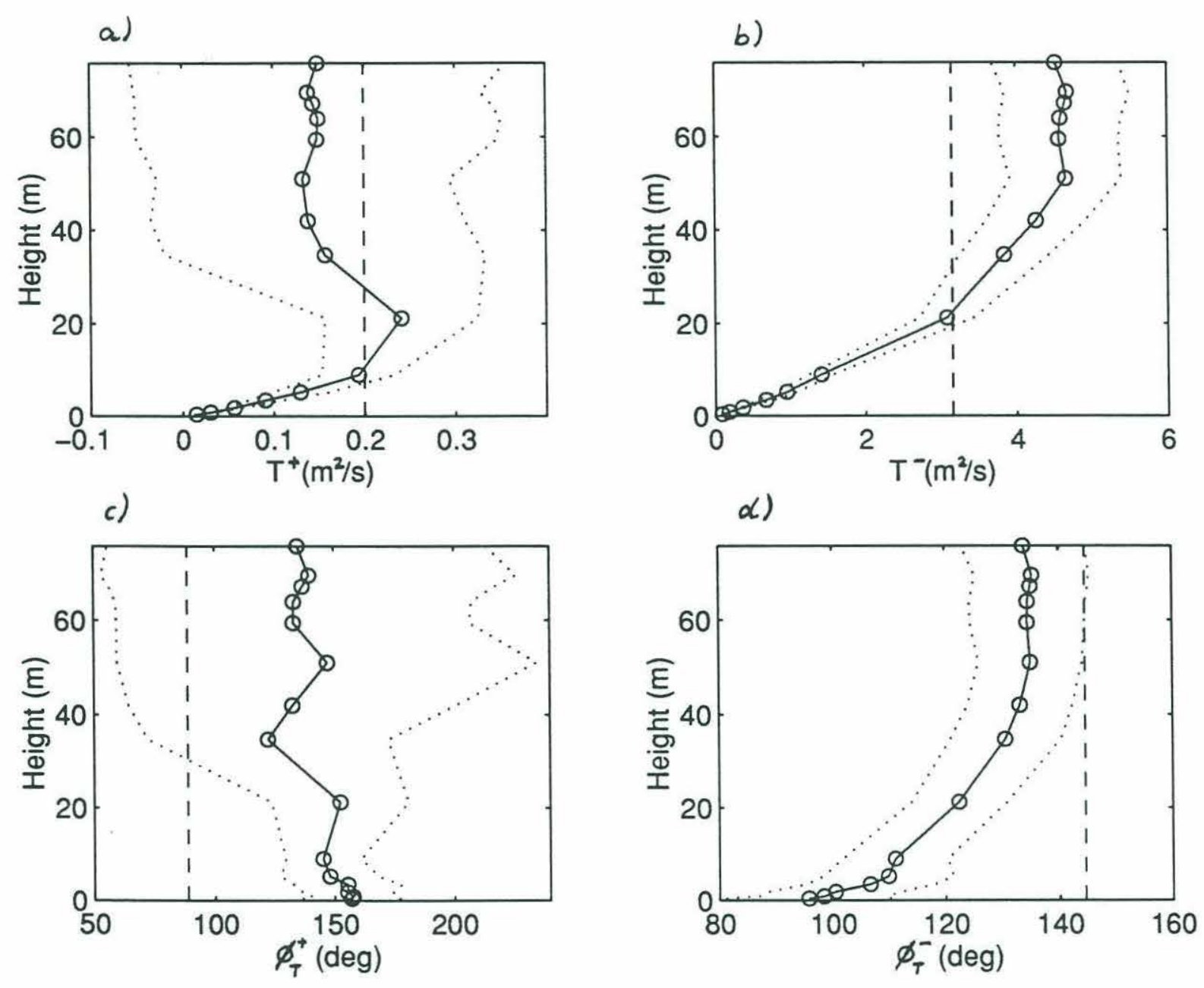

Figure 27: Vertical structure of the integrated velocity defect for the $\mathrm{M}_{2}$ constituent (solid lines), and boundary layer transports predicted by $M_{2}$ bottom stress estimates obtained from BASS data (dashed lines). Profiles show transport magnitudes $T^{+}$(a) and $T^{-}(\mathrm{b})$, and corresponding phase angles $\phi_{T}^{+}(\mathrm{c})$ and $\phi_{T}^{-}(\mathrm{d})$. Dotted lines are error bars. Phase angles describe the orientation of the $\mathrm{M}_{2}$ transport vector at the beginning of the time series and are given with respect to east, where negative values are clockwise from east. 

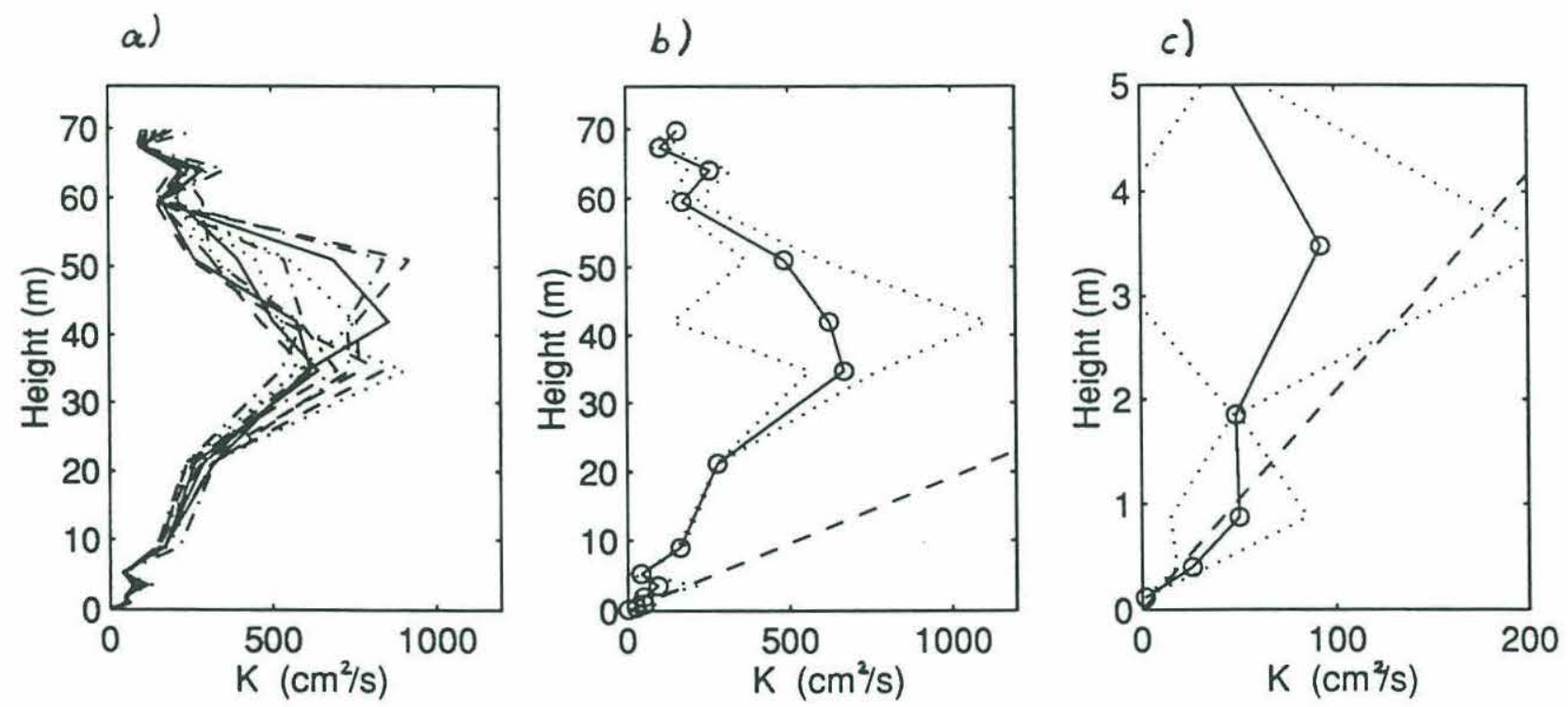

Figure 28: Vertical structure of mean eddy viscosities $K$ calculated for yearday $50-80$. Profiles show the average hourly variation of $K$ during one tidal cycle (a), and the vertical structure of the time mean ( $b$ and $c$ ). Dotted lines in ( $b$ and $c$ ) are error bars. Dashed lines in (b and c) depict the slope $\kappa \bar{u}_{*} z$, where $\bar{u}_{*}=1.2 \mathrm{~cm} / \mathrm{s}$ according to estimates derived from BASS data. 

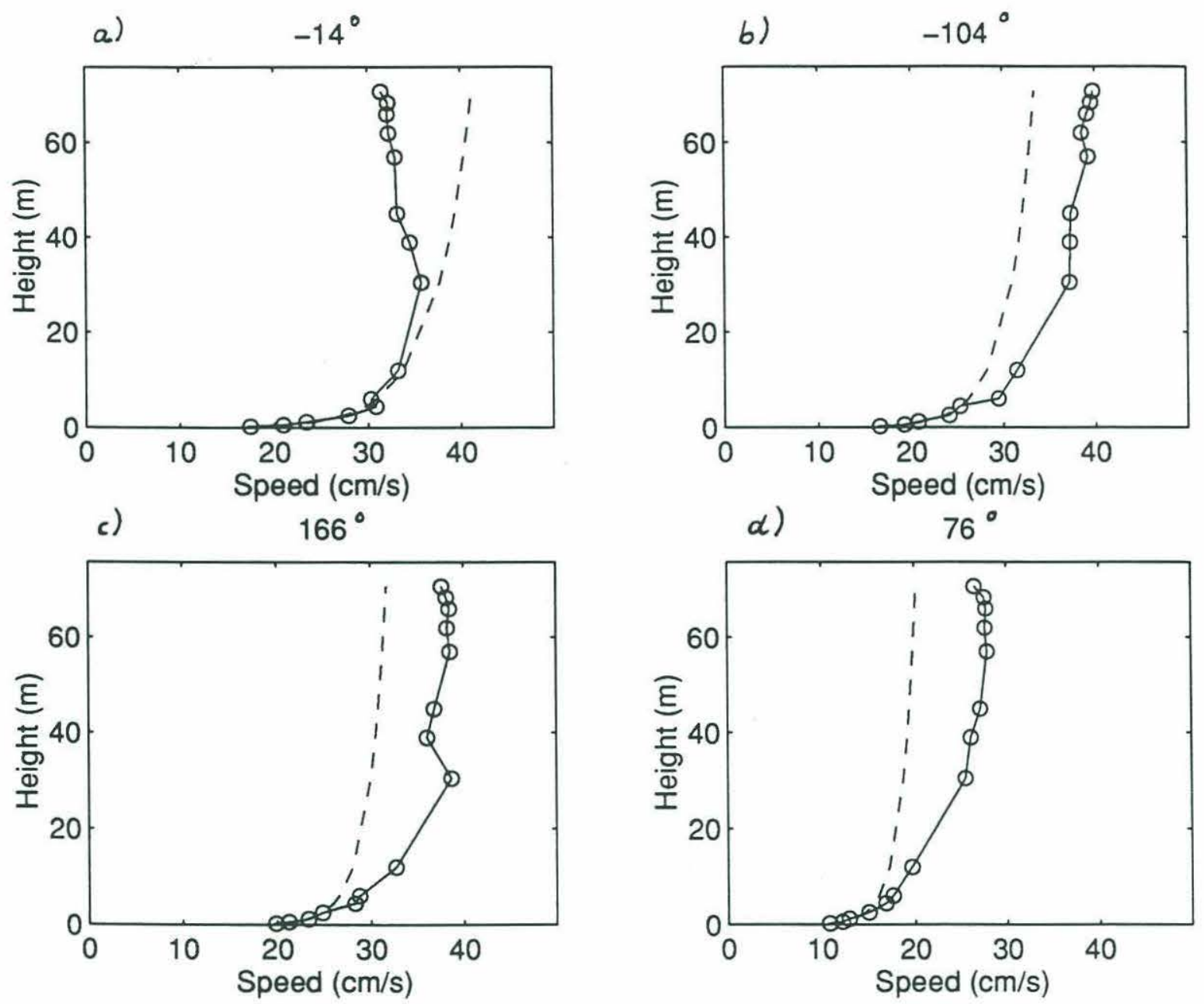

Figure 29: Vertical structure of the total current speeds $U$ from BASS and VMCM data taken between yearday 50-80. Dashed lines represent results from a least-squares logarithmic fit to velocity data at pods 1-4. Profiles are from averaged data, where the average was taken of current measurements with flow directions less than $\pm 5^{\circ}$ from angles of maximum acceleration and deceleration of the near surface tidal speeds. $\frac{\partial U}{\partial t}$ is largest around $-14^{\circ}$ (a) and $166^{\circ}(\mathrm{c})$, and smallest around $-104^{\circ}(\mathrm{b})$ and $76^{\circ}$ (d), where minus signs denote clockwise from east. 


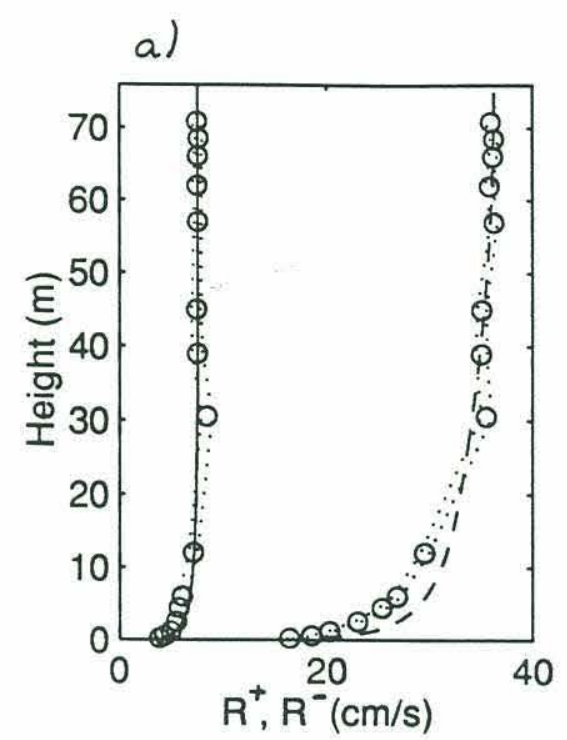

d)

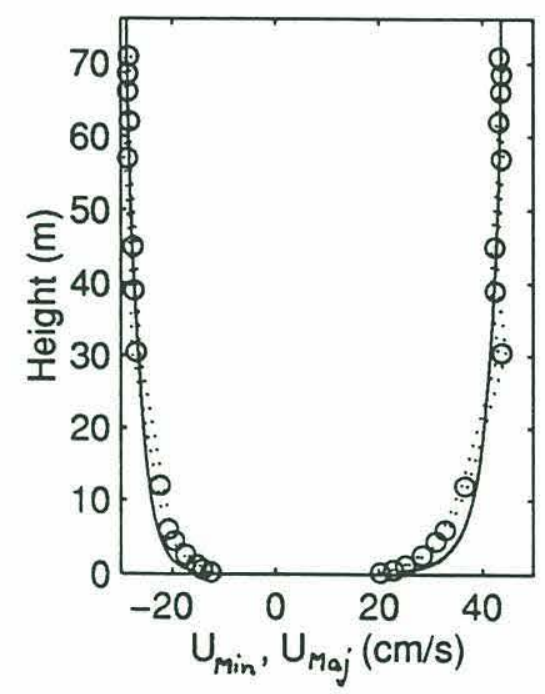

b)

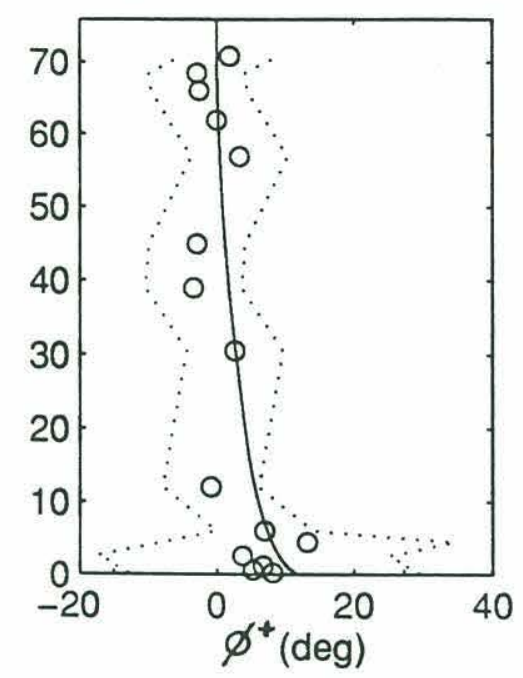

e)

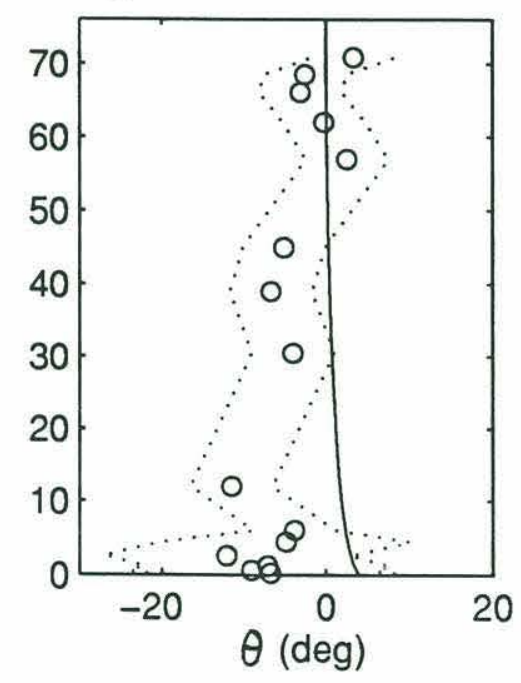

c)

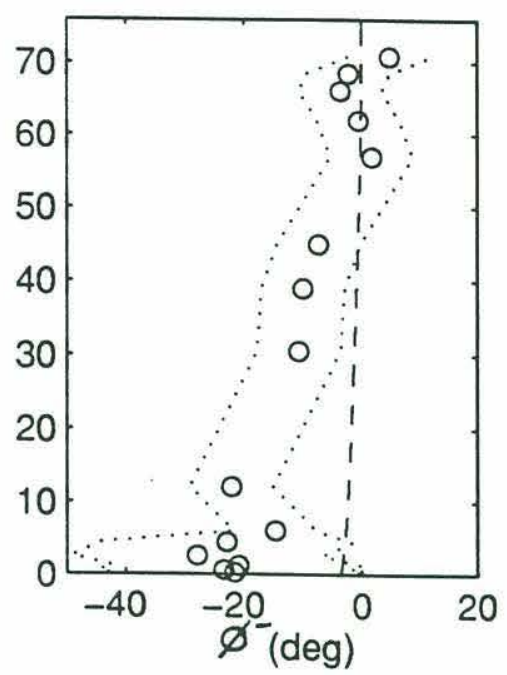

f)

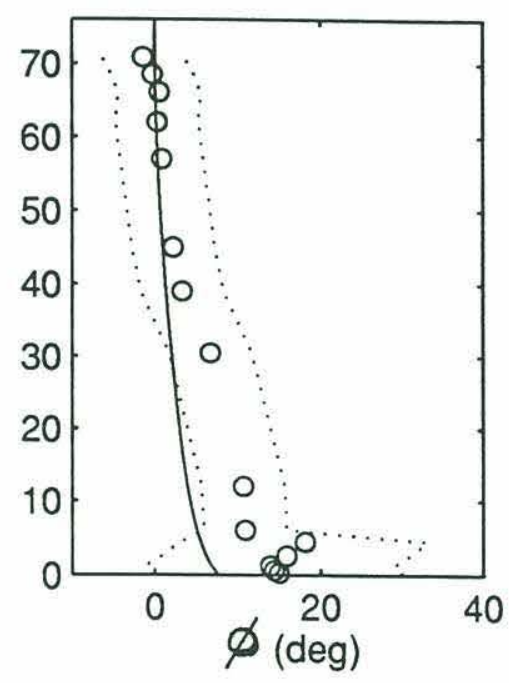

Figure 30: Model results for the vertical structure of the $M_{2}$ current ellipse obtained from the K-profile model with sublayer height $l=\frac{1}{6} \cdot \frac{0.4 \bar{u}_{*}}{\sigma-f}$. Circles represent results obtained from BASS and VMCM measurements, and dotted lines depict the associated uncertainties (Figure 20). Profiles show the magnitudes of the rotary components $R^{+}$(solid line) and $R^{-}$(dashed line) (a), relative veering of the corresponding phase angles $\Delta \phi^{+}$(c) and $\Delta \phi^{-}(\mathrm{d})$, current amplitudes $U_{M a j}$ and $U_{M i n}(\mathrm{~d})$, relative veering of the inclination of the major axis $\Delta \theta$ (e) and of the phase of the velocity vector $\Delta \phi(\mathrm{f})$. Decreasing angles $\Delta \theta$ and $\Delta \phi^{ \pm}$depict clockwise rotation of the major axis $U_{M a j}$ and rotary components $R^{ \pm}$, respectively. Decreasing angles $\Delta \phi$ represent phase lag. 


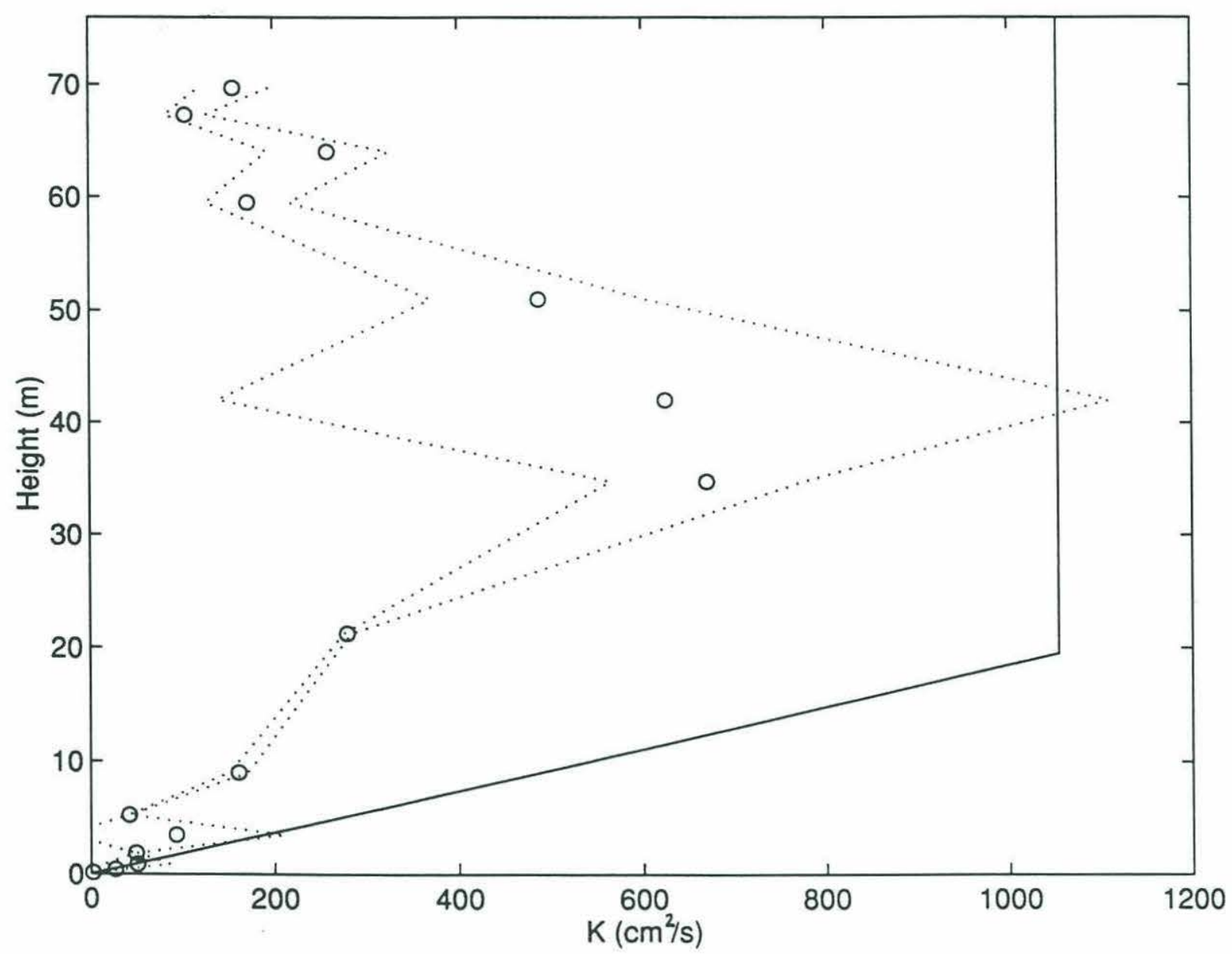

Figure 31: Numerical eddy viscocities $K$ obtained from the K-profile model with sublayer height $l=\frac{1}{6} \cdot \frac{0.4 \bar{u}_{0}}{\sigma-f}$ (solid line). Circles represent results from measured data, and dotted lines are the corresponding uncertainties (Figure 28). In the sublayer, $K=\kappa \bar{u}_{*} z$, where $\bar{u}_{*}=1.35 \mathrm{~cm} / \mathrm{s}$ according to model results. 
a)

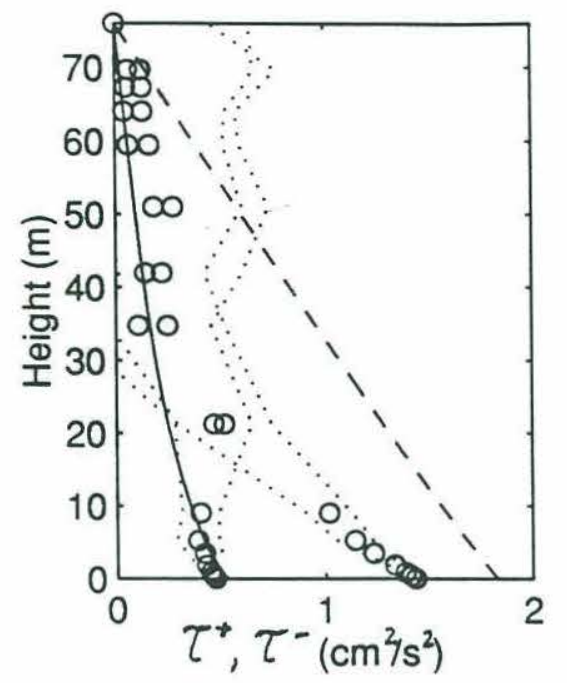

d)

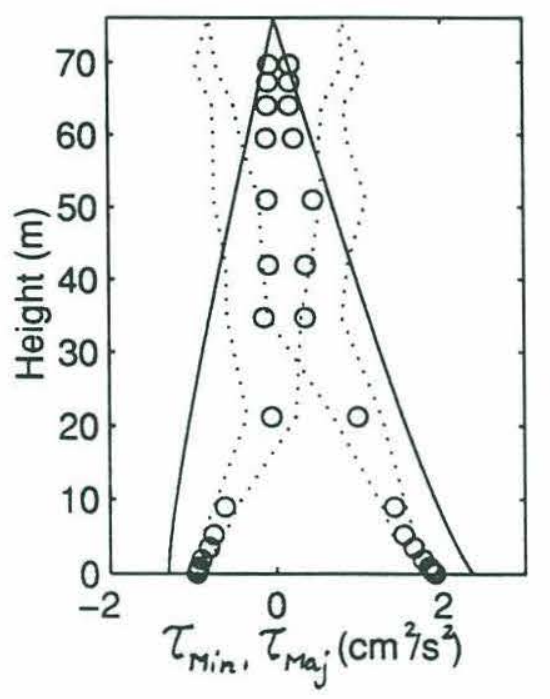

b)

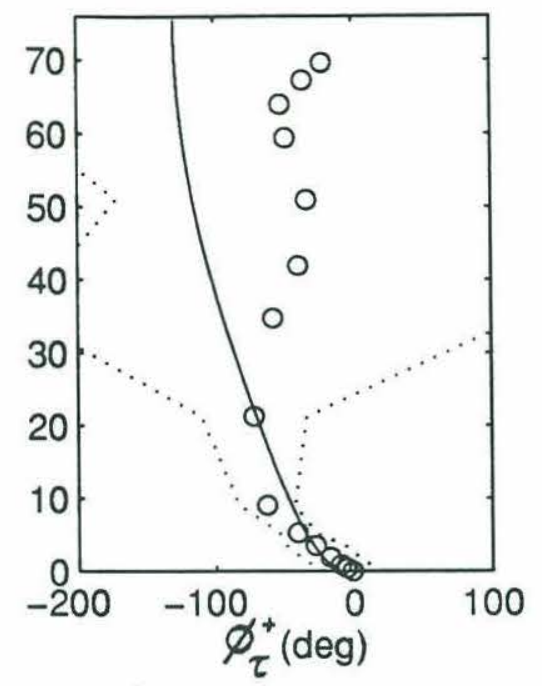

e)

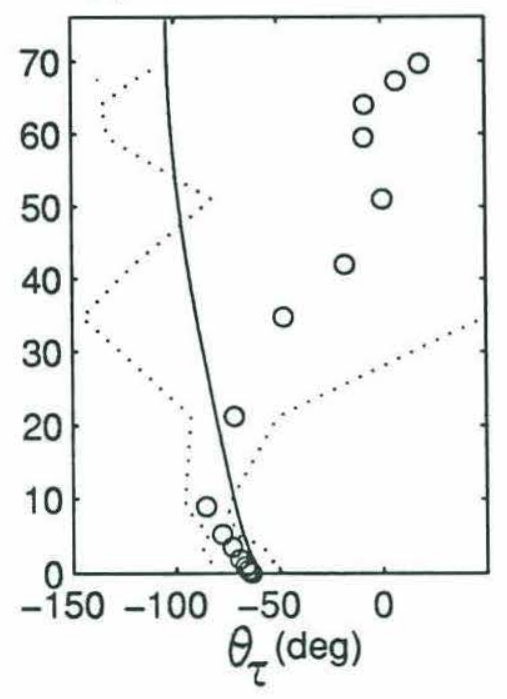

c)
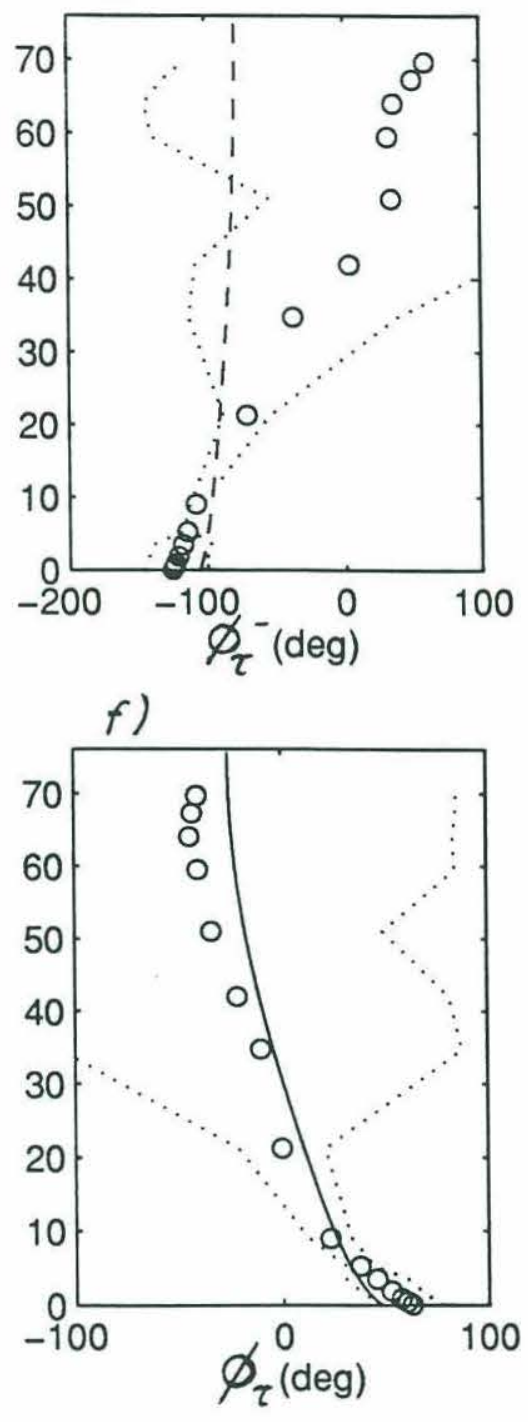

Figure 32: Model results for the vertical structure of the $M_{2}$ stress ellipse obtained from the K-profile model with sublayer height $l=\frac{1}{6} \cdot \frac{0.4 \bar{u}_{0}}{\sigma-f}$. Circles represent results from actual data, and dotted lines are the associated uncertainties (Figure 25). Profiles show the magnitudes of the rotary components $\tau^{+}$(solid line) and $\tau^{-}$(dashed line) (a), corresponding phase angles $\phi_{\tau}^{+}(\mathrm{c})$ and $\phi_{\tau}^{-}(\mathrm{d})$, current amplitudes $\tau_{M a j}$ and $\tau_{M i n}(\mathrm{~d})$, inclination of the major axis $\theta_{\tau}(\mathrm{e})$, and phase of the stress vector $\phi_{\tau}(\mathrm{f})$. Inclination and phase angles of the rotary components are given with respect to east, where negative values are clockwise from east. Phase angles describe the orientation of the $\mathrm{M}_{2}$ stress vector at the beginning of the time series. 
a)

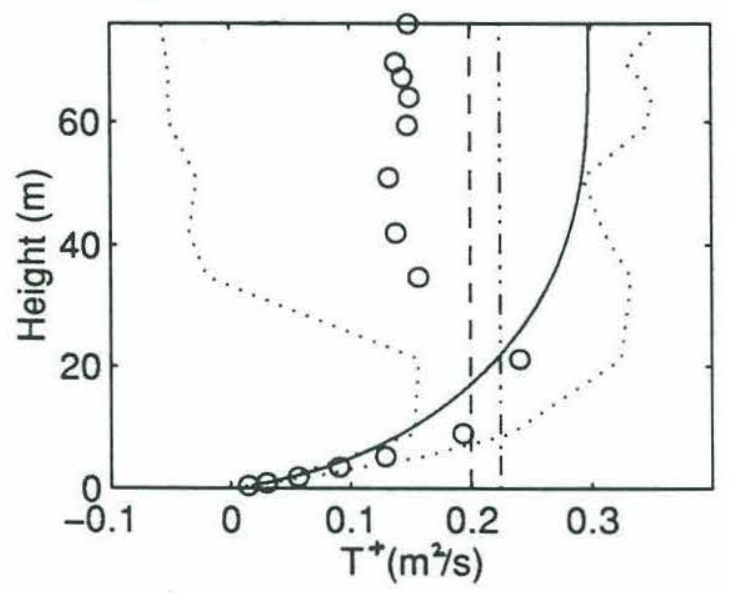

c)

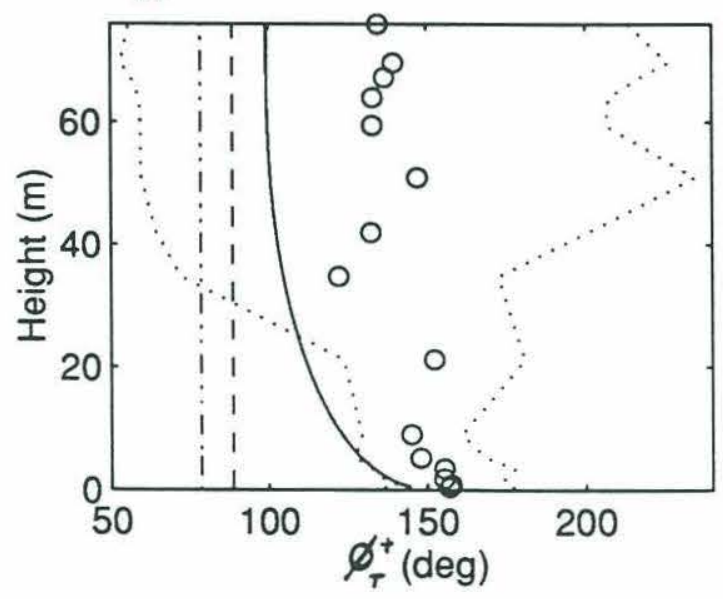

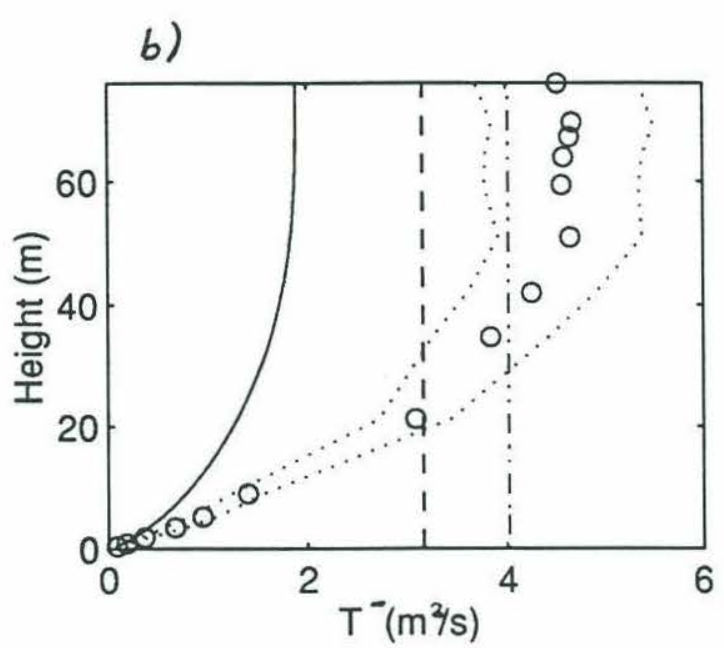

d)

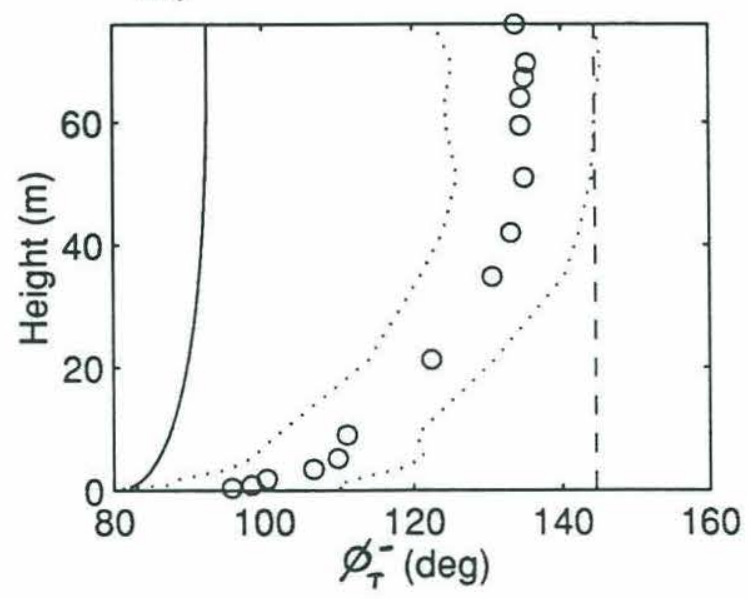

Figure 33: Model results for the integrated velocity defect of the $M_{2}$ constituent (solid lines), and tidal boundary layer transports predicted by numerical bottom stresses (dotdashed lines) obtained from the K-profile model with sublayer height $l=\frac{1}{6} \cdot \frac{0.4 \bar{u}_{\text {. }}}{\sigma-f}$. Circles represent results from velocity defects of actual data, and dashed lines are boundary layer transports predicted by BASS bottom stress estimates (Figure 27). Dotted lines are uncertainties associated with the data. Profiles show transport magnitudes $T^{+}$(a) and $T^{-}(\mathrm{b})$, and corresponding phase angles $\phi_{T}^{+}$(c) and $\phi_{T}^{-}(\mathrm{d})$. Phase angles describe the orientation of the $\mathrm{M}_{2}$ transport vector at the beginning of the time series and are given with respect to east, where negative values are clockwise from east. 
a)

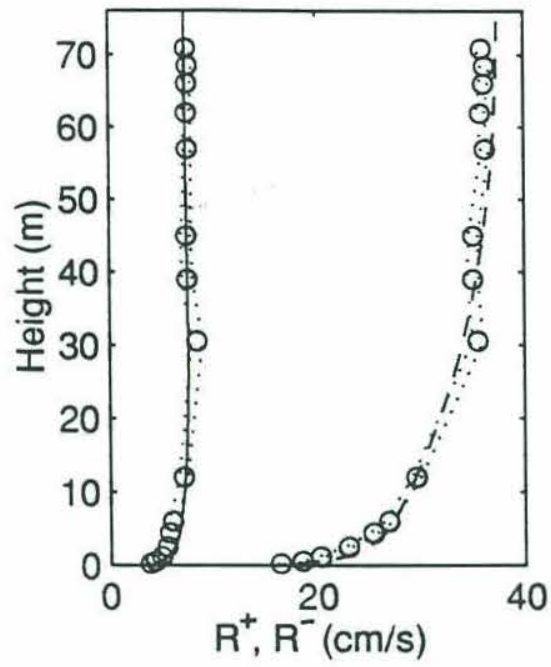

d)

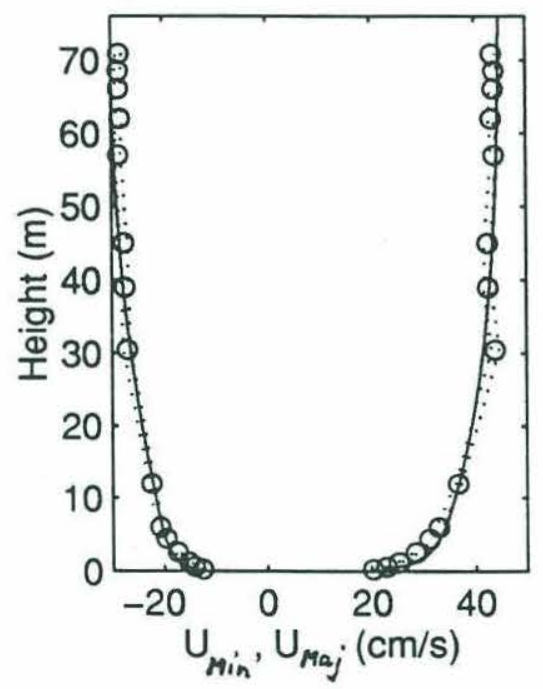

b)

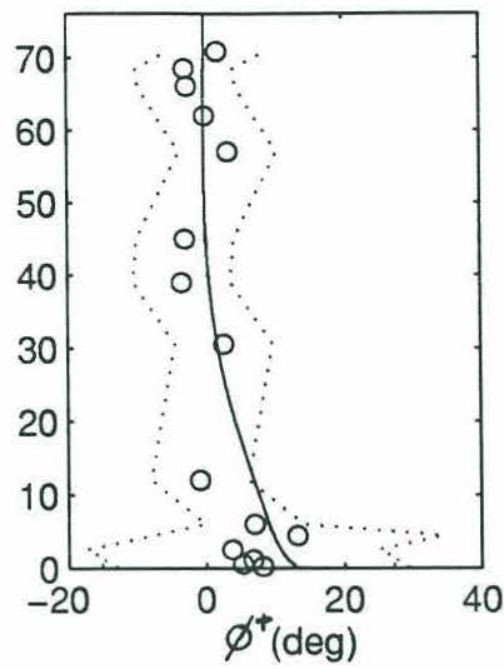

e)

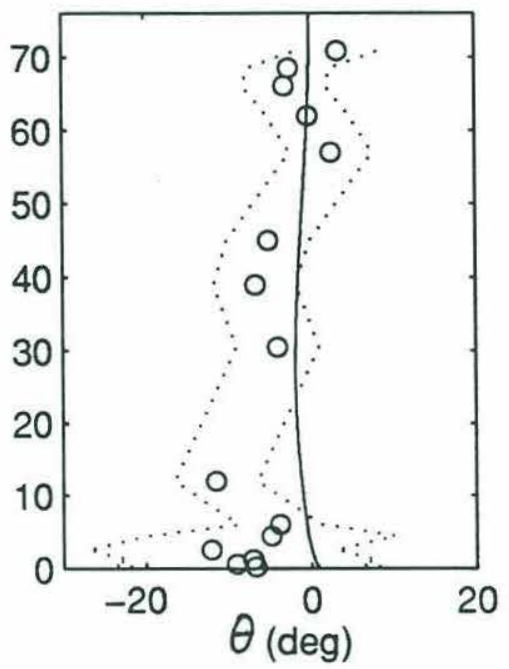

c)

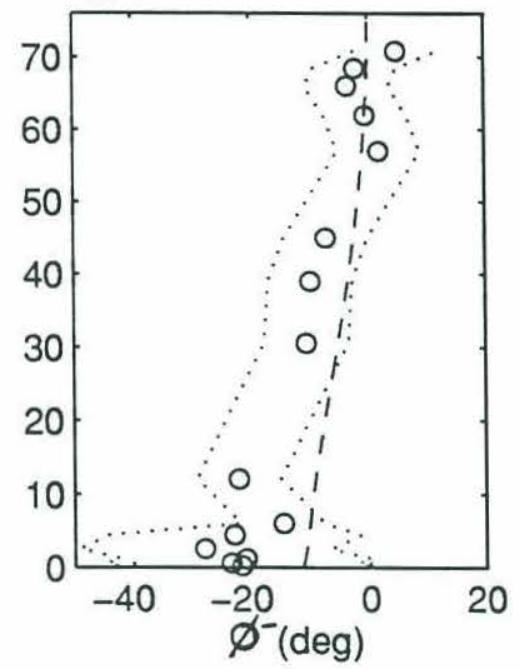

f)

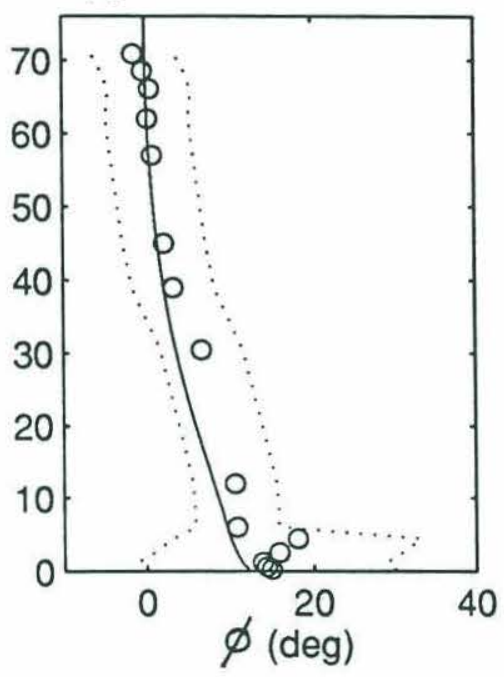

Figure 34: Model results for the vertical structure of the $M_{2}$ current ellipse obtained from the K-profile model with sublayer height $l=\frac{1}{6} \cdot 0.39 \frac{\kappa \bar{u}_{0}}{(\sigma-f)}$. Circles represent results obtained from BASS and VMCM measurements, and dotted lines depict the associated uncertainties (Figure 20). Profiles show the magnitudes of the rotary components $R^{+}$(solid line) and $R^{-}$(dashed line) (a), relative veering of the corresponding phase angles $\Delta \phi^{+}$(c) and $\Delta \phi^{-}(\mathrm{d})$, current amplitudes $U_{M a j}$ and $U_{M i n}(\mathrm{~d})$, relative veering of the inclination of the major axis $\Delta \theta$ (e) and of the phase of the velocity vector $\Delta \phi(\mathrm{f})$. Decreasing angles $\Delta \theta$ and $\Delta \phi^{ \pm}$depict clockwise rotation of the major axis $U_{M a j}$ and rotary components $R^{ \pm}$, respectively. Decreasing angles $\Delta \phi$ represent phase lag. 


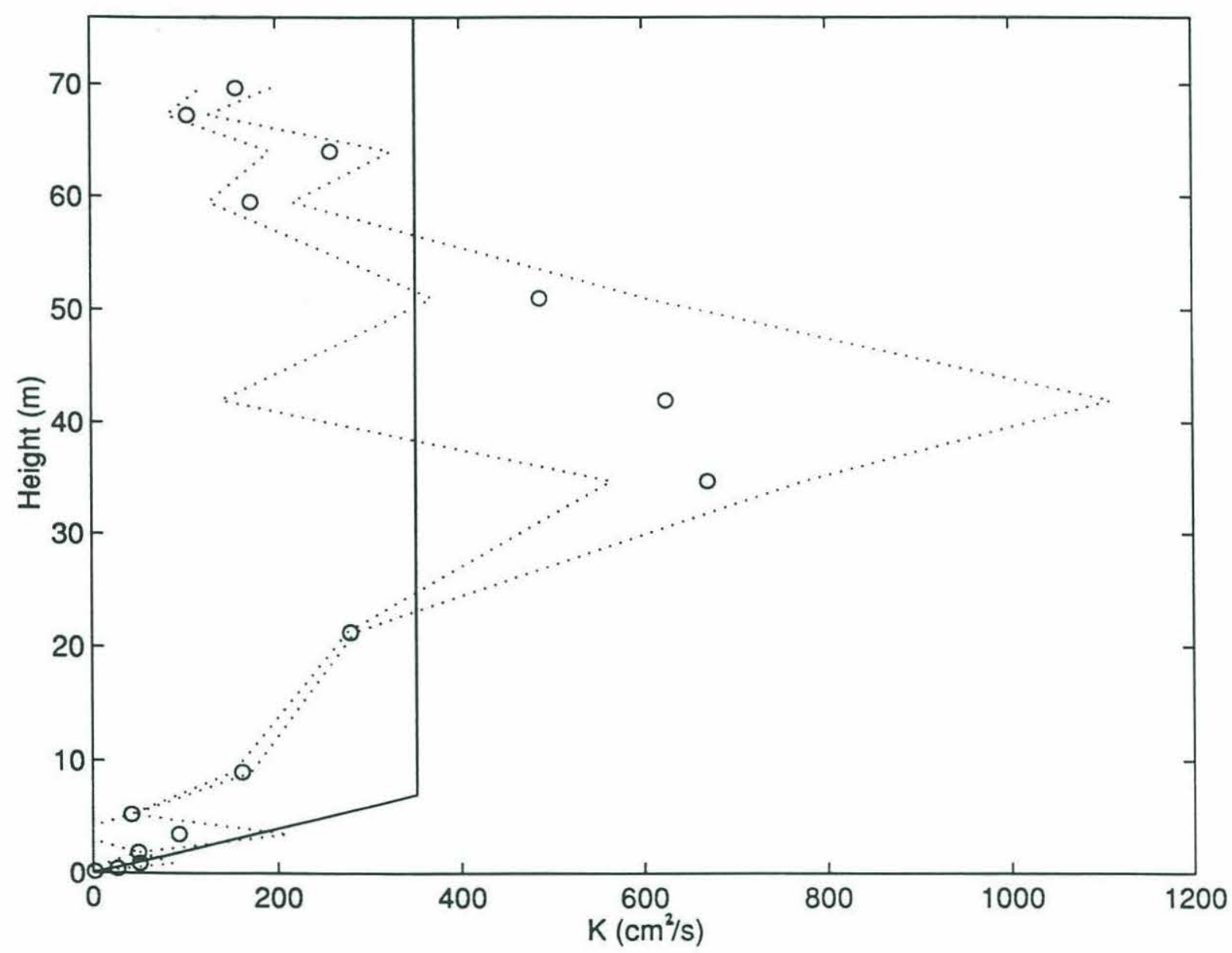

Figure 35: Numerical eddy viscocities $K$ obtained from the K-profile model with sublayer height $l=\frac{1}{6} \cdot 0.39 \frac{\kappa \bar{u}_{0}}{(\sigma-f)}$ (solid line). Circles represent results from measured data, and dotted lines are the corresponding uncertainties (Figure 28). In the sublayer, $K=\kappa \bar{u}_{*} z$, where $\bar{u}_{*}=1.25 \mathrm{~cm} / \mathrm{s}$ according to model results. 
a)

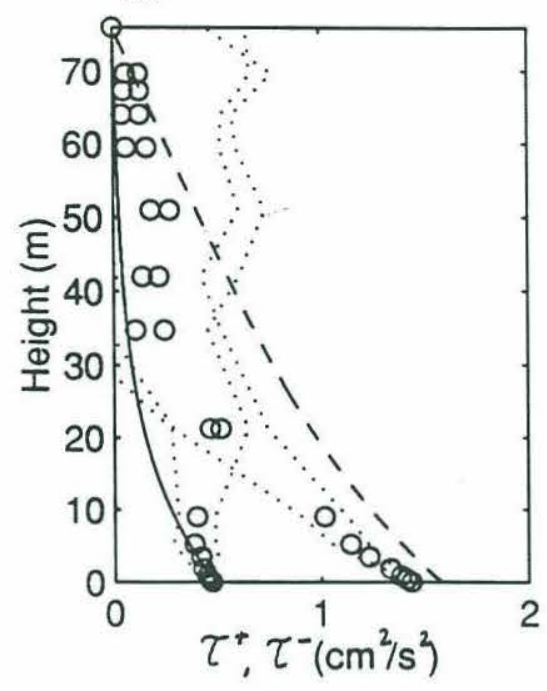

d)

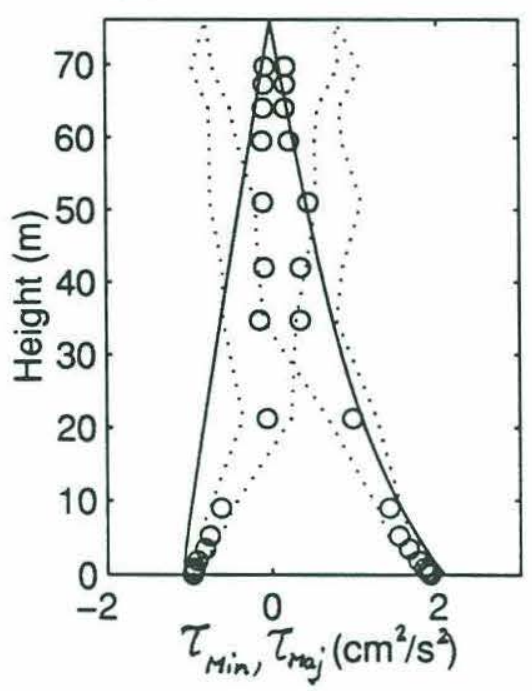

b)

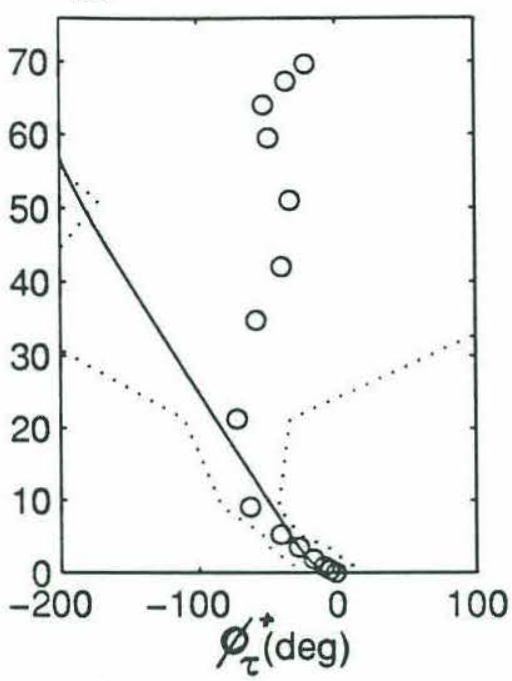

e)

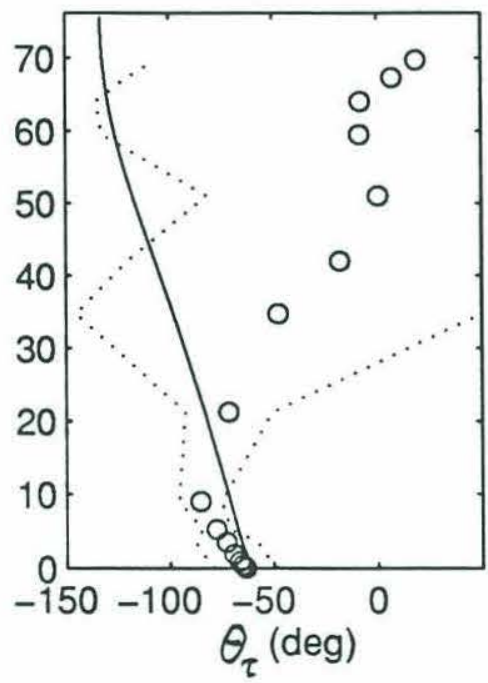

c)

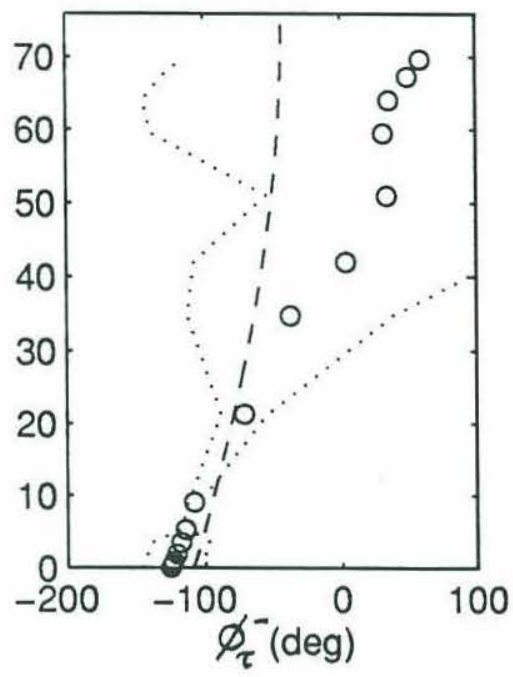

f)

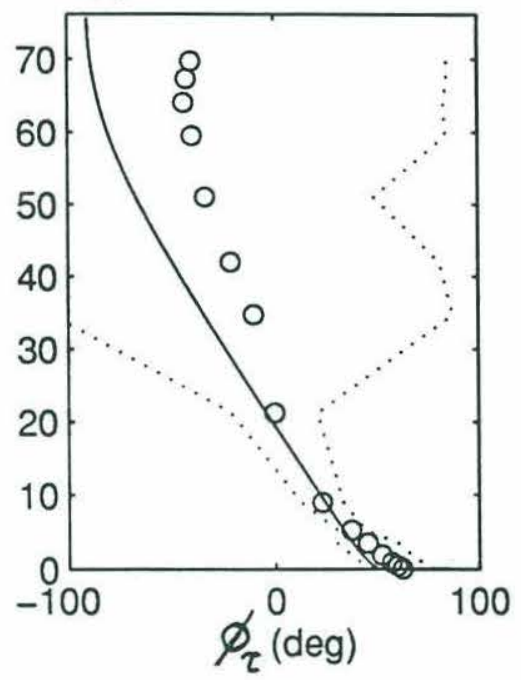

Figure 36: Model results for the vertical structure of the $M_{2}$ stress ellipse obtained from the K-profile model with sublayer height $l=\frac{1}{6} \cdot 0.39 \frac{\kappa \bar{u}_{*}}{(\sigma-f)}$. Circles represent results from actual data, and dotted lines are the associated uncertainties (Figure 25). Profiles show the magnitudes of the rotary components $\tau^{+}$(solid line) and $\tau^{-}$(dashed line) (a), corresponding phase angles $\phi_{\tau}^{+}(\mathrm{c})$ and $\phi_{\tau}^{-}(\mathrm{d})$, current amplitudes $\tau_{M a j}$ and $\tau_{M \text { in }}(\mathrm{d})$, inclination of the major axis $\theta_{\tau}(\mathrm{e})$, and phase of the stress vector $\phi_{\tau}$ (f). Inclination and phase angles of the rotary components are given with respect to east, where negative values are clockwise from east. Phase angles describe the orientation of the $M_{2}$ stress vector at the beginning of the time series. 
a)

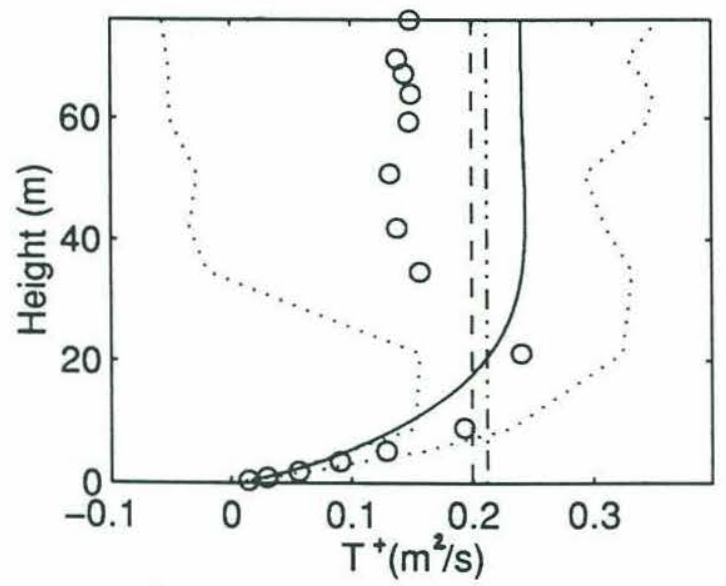

c)

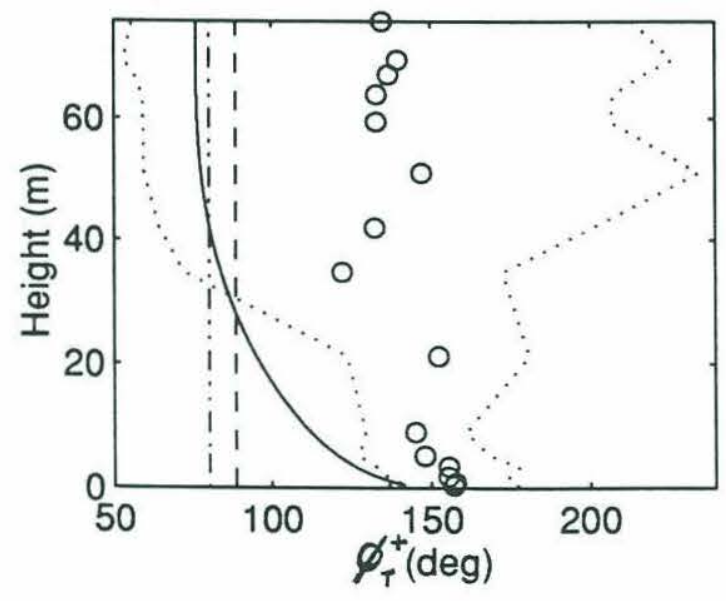

b)

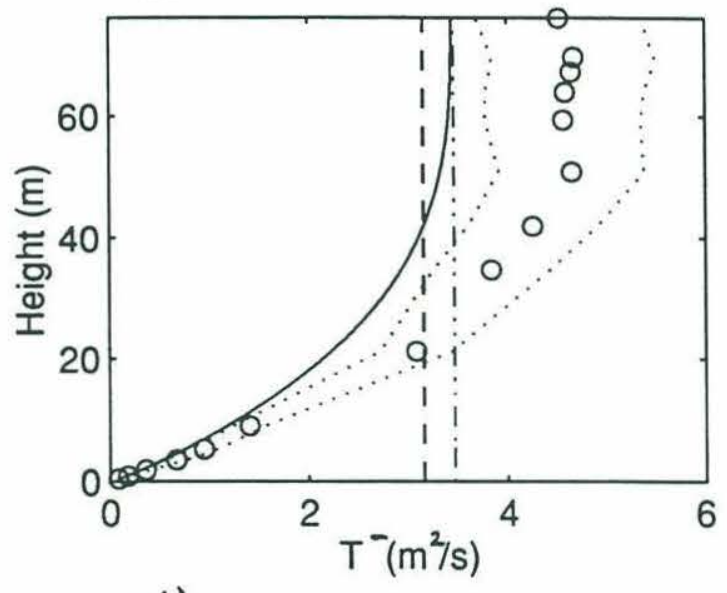

d)

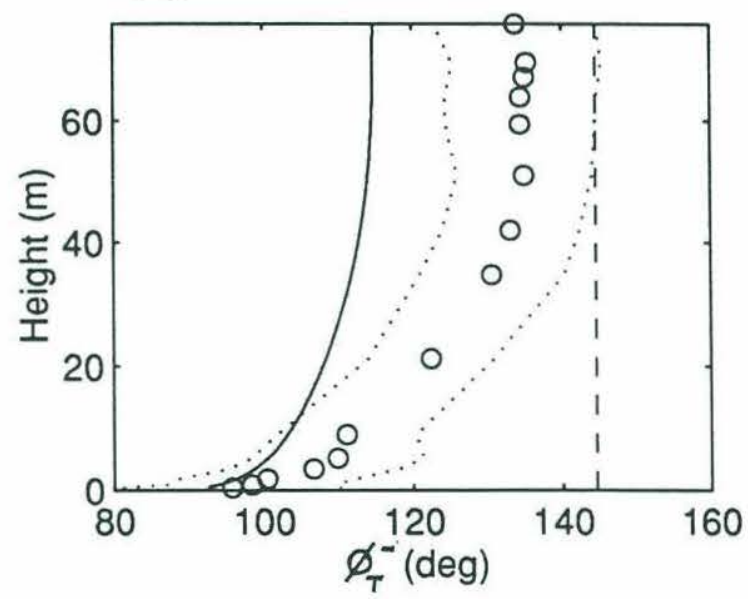

Figure 37: Model results for the integrated velocity defect of the $M_{2}$ constituent (solid lines), and tidal boundary layer transports predicted by numerical bottom stresses (dotdashed lines) obtained from the K-profile model with sublayer height $l=\frac{1}{6} \cdot 0.39 \frac{\kappa \bar{u} \text {. }}{(\sigma-f)}$. Circles represent results from velocity defects of actual data, and dashed lines are boundary layer transports predicted by BASS bottom stress estimates (Figure 27). Dotted lines are uncertainties associated with the data. Profiles show transport magnitudes $T^{+}(\mathrm{a})$ and $T^{-}$(b), and corresponding phase angles $\phi_{T}^{+}$(c) and $\phi_{T}^{-}$(d). Phase angles describe the orientation of the $\mathrm{M}_{2}$ transport vector at the beginning of the time series and are given with respect to east, where negative values are clockwise from east. 
a)

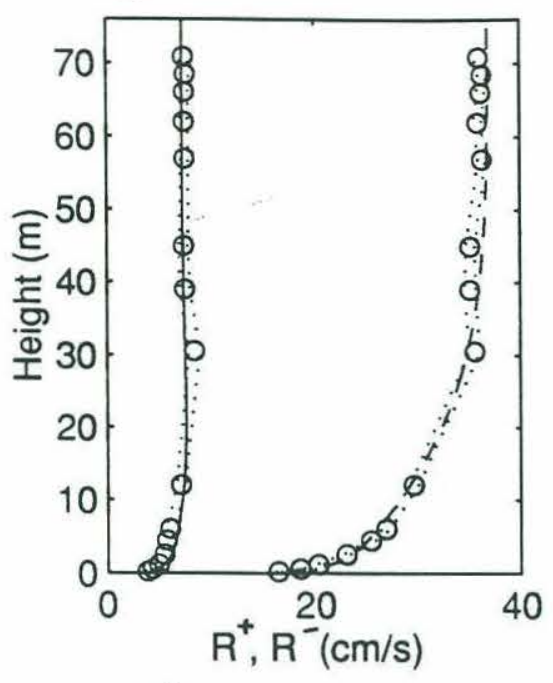

d)

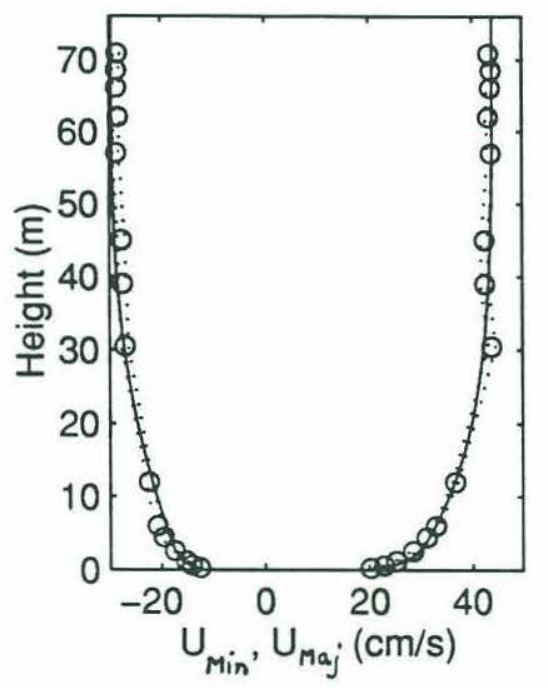

b)

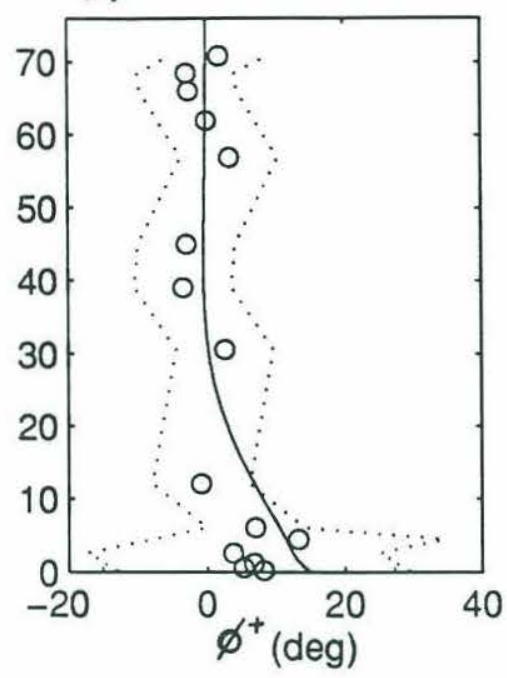

e)

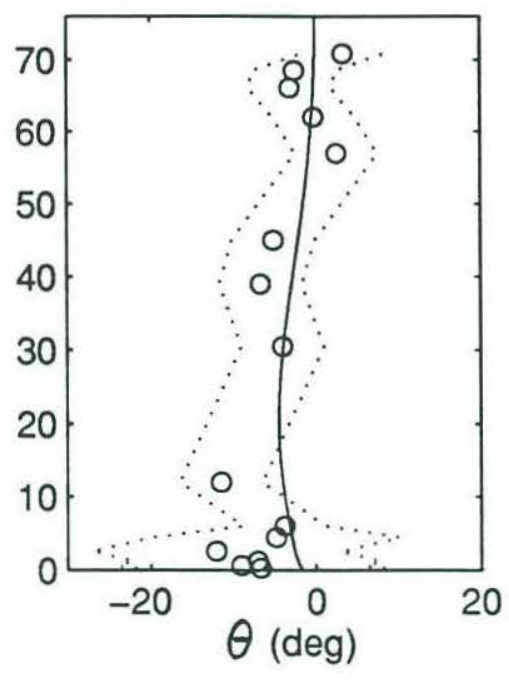

c)

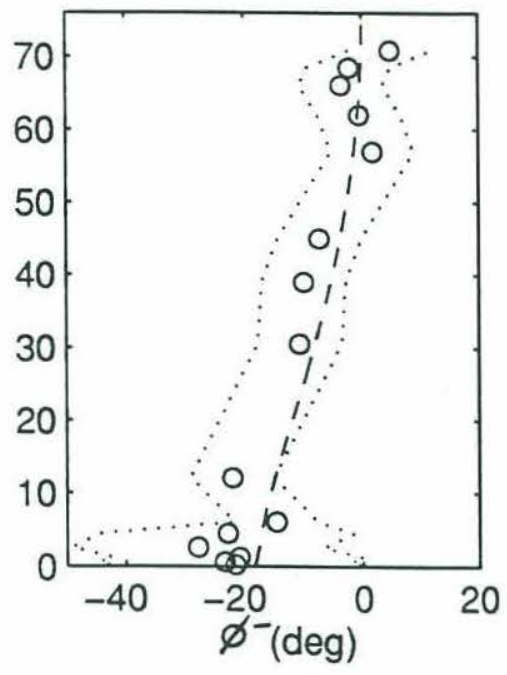

f)

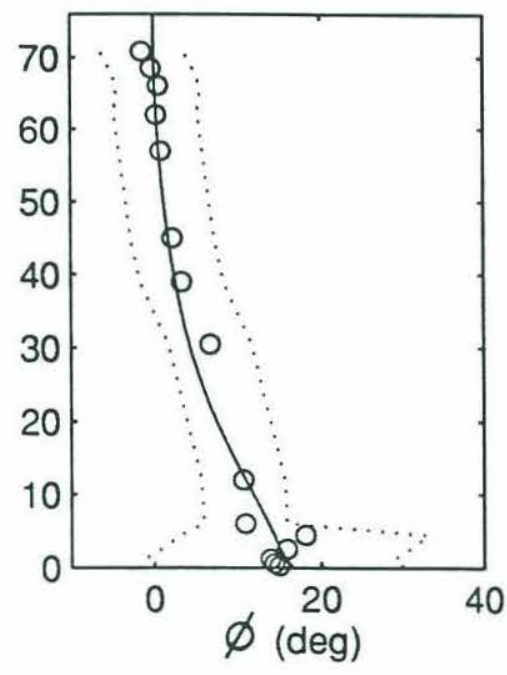

Figure 38: Model results for the vertical structure of the $M_{2}$ current ellipse obtained from the K-profile model with sublayer height $l=\frac{1}{10} \cdot 0.39 \frac{\kappa \bar{u}_{*}}{(\sigma-f)}$. Circles represent results obtained from BASS and VMCM measurements, and dotted lines depict the associated uncertainties (Figure 20). Profiles show the magnitudes of the rotary components $R^{+}$(solid line) and $R^{-}$(dashed line) (a), relative veering of the corresponding phase angles $\Delta \phi^{+}$(c) and $\Delta \phi^{-}(\mathrm{d})$, current amplitudes $U_{M a j}$ and $U_{M i n}(\mathrm{~d})$, relative veering of the inclination of the major axis $\Delta \theta(\mathrm{e})$ and of the phase of the velocity vector $\Delta \phi(\mathrm{f})$. Decreasing angles $\Delta \theta$ and $\Delta \phi^{ \pm}$depict clockwise rotation of the major axis $U_{M a j}$ and rotary components $R^{ \pm}$, respectively. Decreasing angles $\Delta \phi$ represent phase lag. 


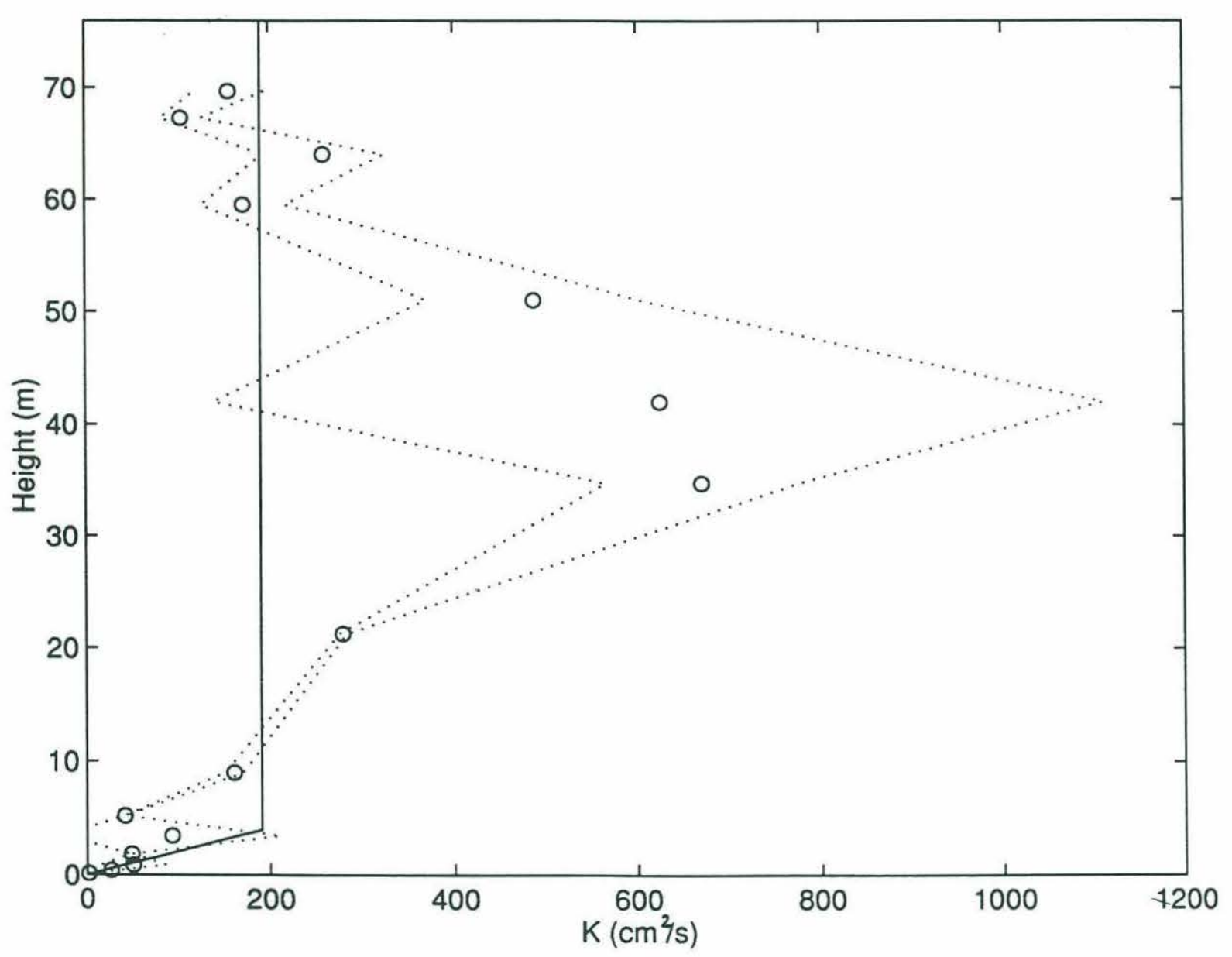

Figure 39: Numerical eddy viscocities $K$ obtained from the K-profile model with sublayer height $l=\frac{1}{10} \cdot 0.39 \frac{\kappa \bar{u}_{*}}{(\sigma-f)}$ (solid line). Circles represent results from measured data, and dotted lines are the corresponding uncertainties (Figure 28). In the sublayer, $K=\kappa \bar{u}_{*} z$, where $\bar{u}_{*}=1.19 \mathrm{~cm} / \mathrm{s}$ according to model results. 
a)

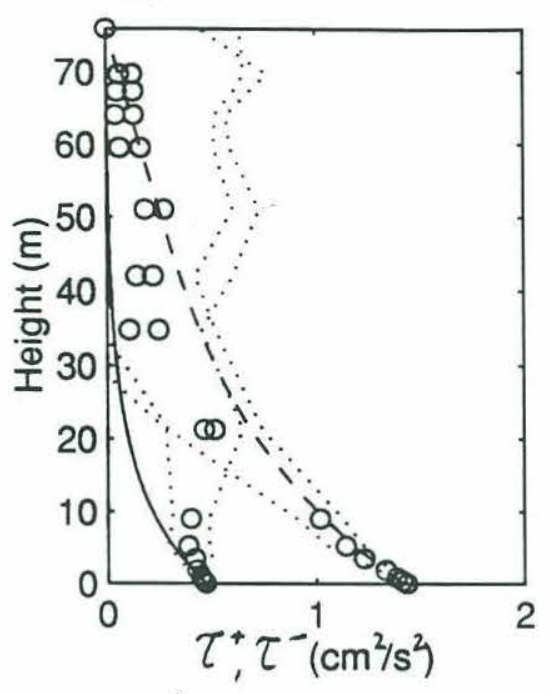

d)

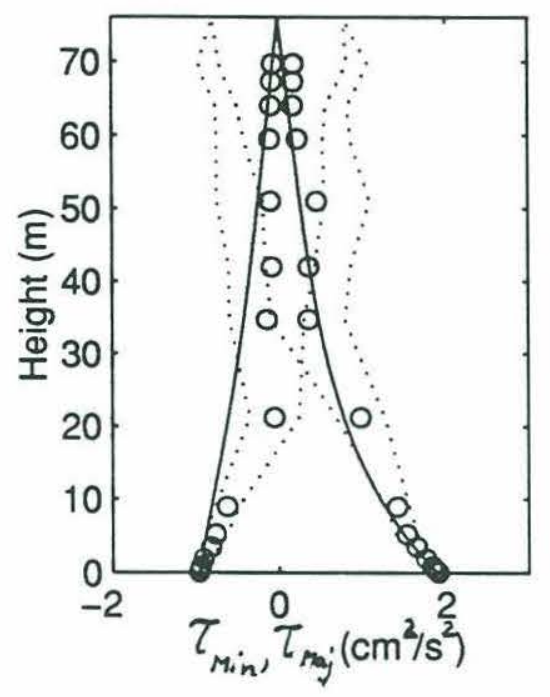

b)

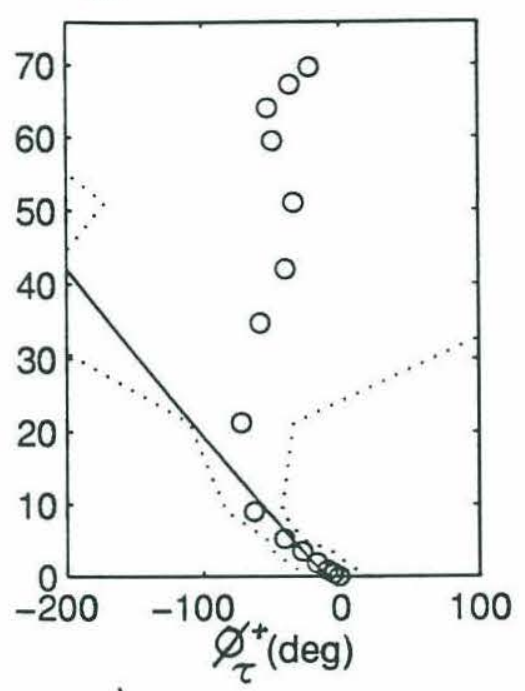

e)

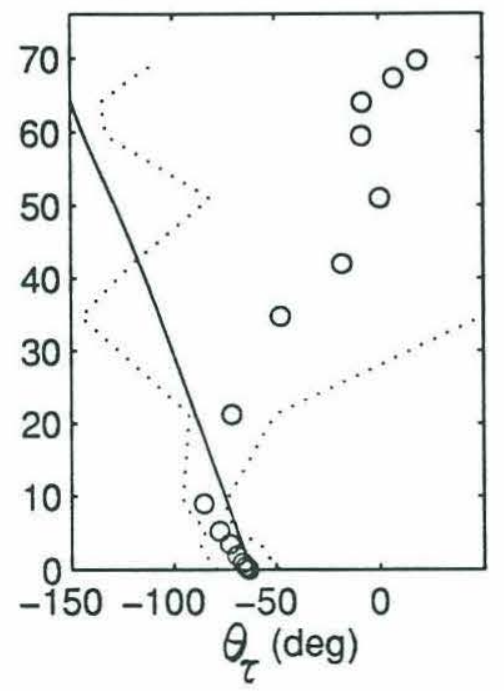

c)
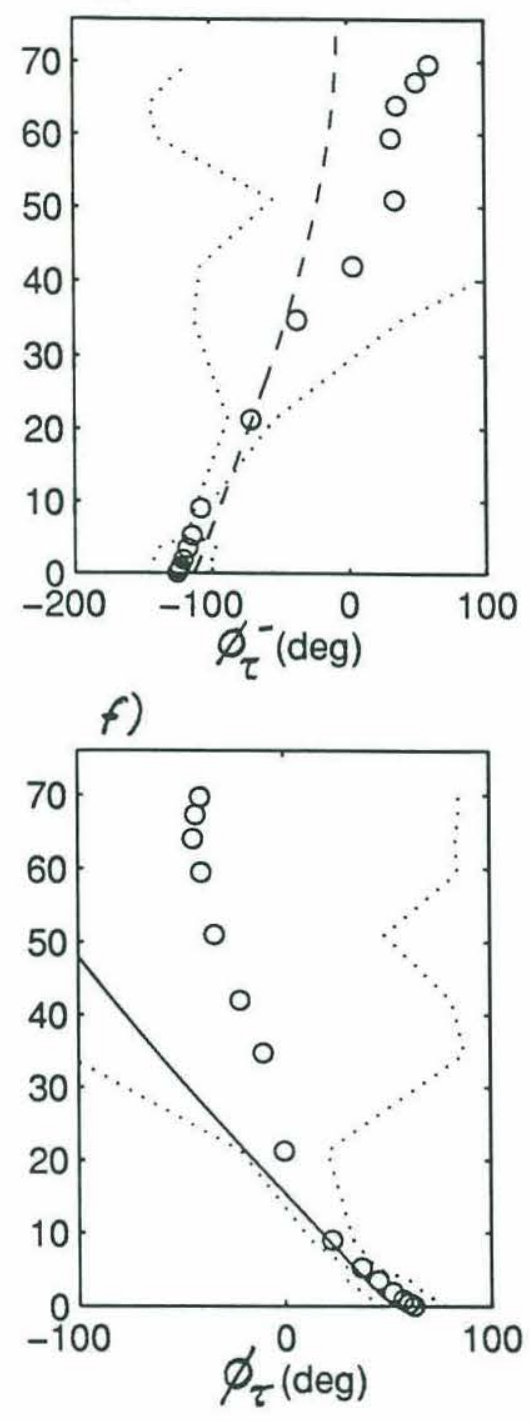

Figure 40: Model results for the vertical structure of the $M_{2}$ stress ellipse obtained from the K-profile model with sublayer height $l=\frac{1}{10} \cdot 0.39 \frac{\kappa \bar{u}_{\circ}}{(\sigma-f)}$. Circles represent results from actual data, and dotted lines are the associated uncertainties (Figure 25). Profiles show the magnitudes of the rotary components $\tau^{+}$(solid line) and $\tau^{-}$(dashed line) (a), corresponding phase angles $\phi_{\tau}^{+}(\mathrm{c})$ and $\phi_{\tau}^{-}(\mathrm{d})$, current amplitudes $\tau_{M a j}$ and $\tau_{M \text { in }}(\mathrm{d})$, inclination of the major axis $\theta_{\tau}(\mathrm{e})$, and phase of the stress vector $\phi_{\tau}$ (f). Inclination and phase angles of the rotary components are given with respect to east, where negative values are clockwise from east. Phase angles describe the orientation of the $M_{2}$ stress vector at the beginning of the time series. 
a)

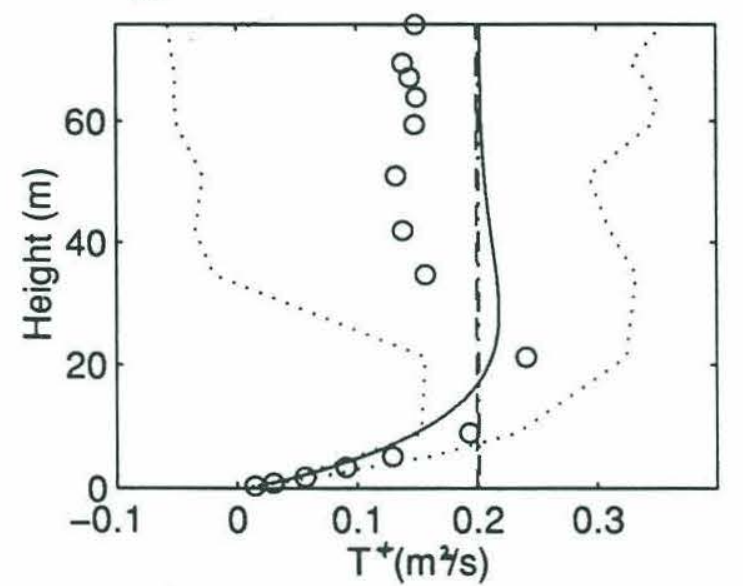

c)

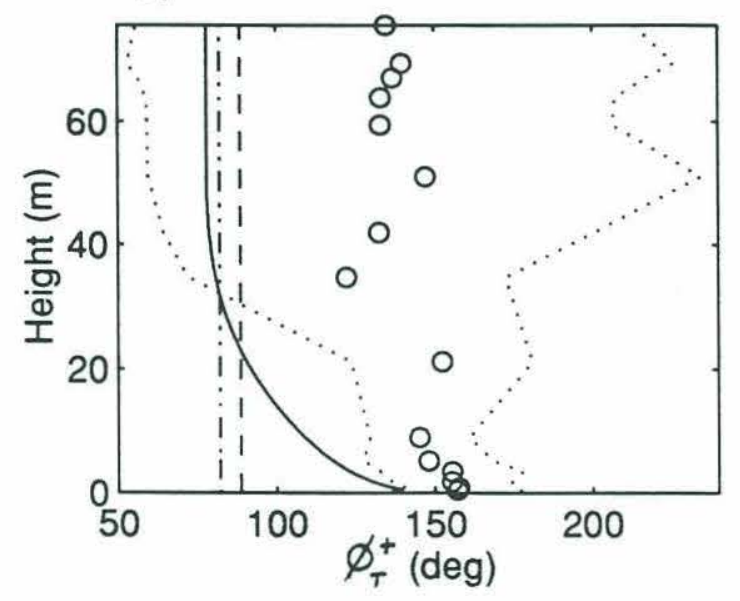

b)

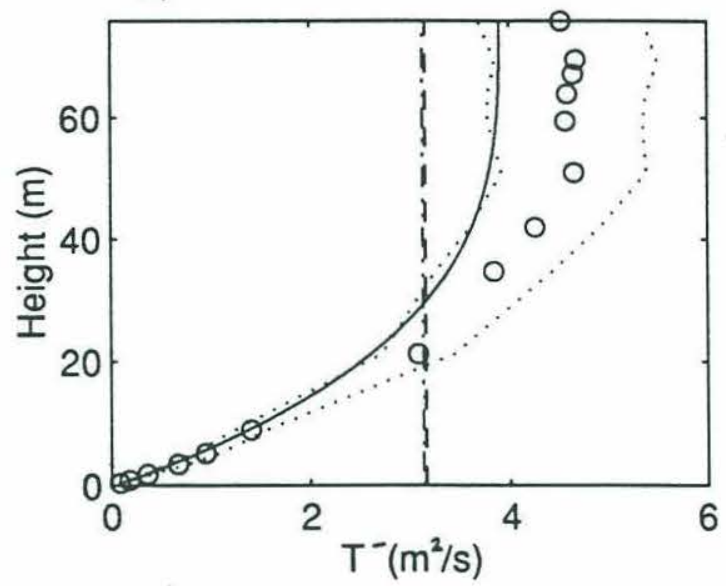

d)

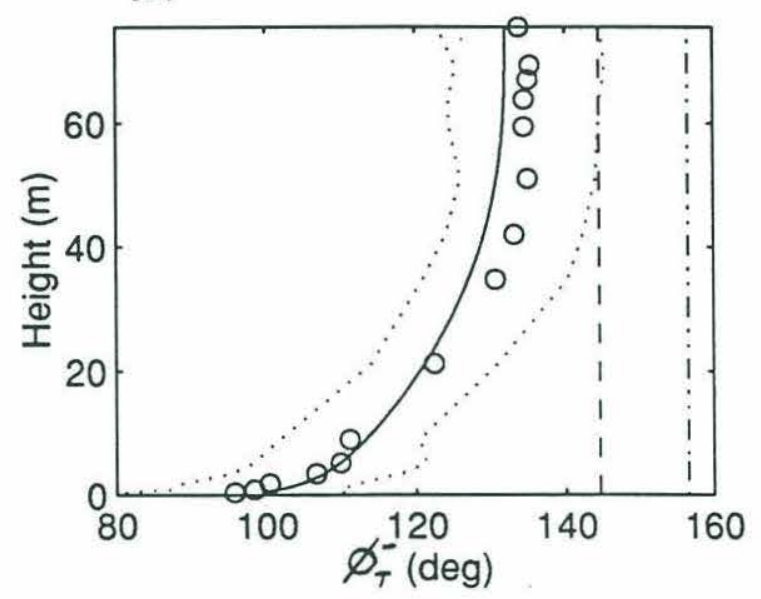

Figure 41: Model results for the integrated velocity defect of the $M_{2}$ constituent (solid lines), and tidal boundary layer transports predicted by numerical bottom stresses (dotdashed lines) obtained from the K-profile model with sublayer height $l=\frac{1}{10} \cdot 0.39 \frac{\kappa \bar{u}}{(\sigma-f)}$. Circles represent results from velocity defects of actual data, and dashed lines are boundary layer transports predicted by BASS bottom stress estimates (Figure 27). Dotted lines are uncertainties associated with the data. Profiles show transport magnitudes $T^{+}(\mathrm{a})$ and $T^{-}$(b), and corresponding phase angles $\phi_{T}^{+}$(c) and $\phi_{T}^{-}$(d). Phase angles describe the orientation of the $M_{2}$ transport vector at the beginning of the time series and are given with respect to east, where negative values are clockwise from east. 
a)

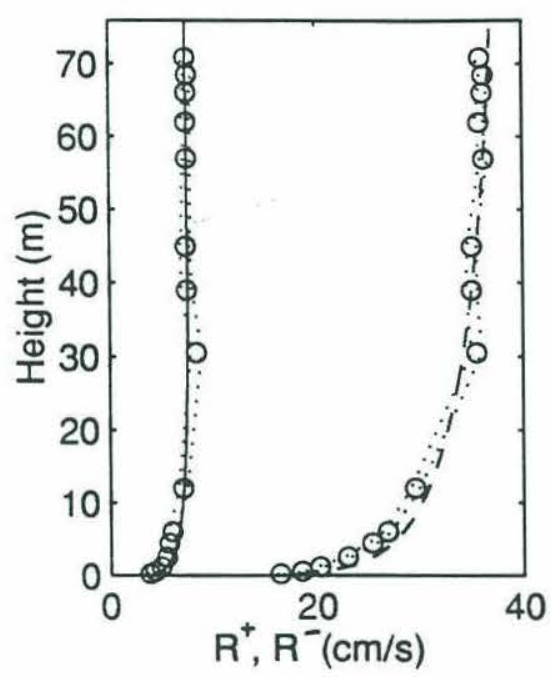

d)

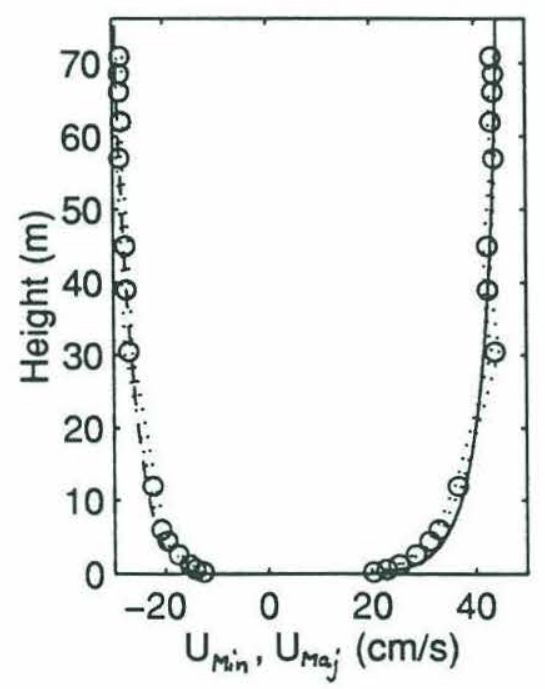

b)

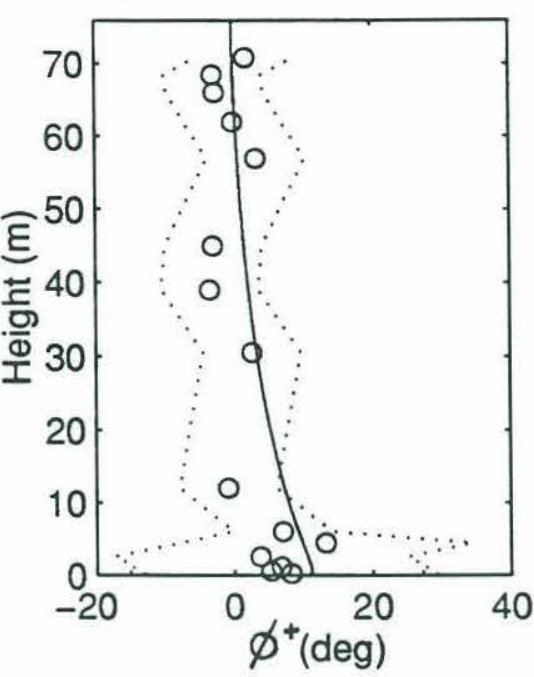

e)

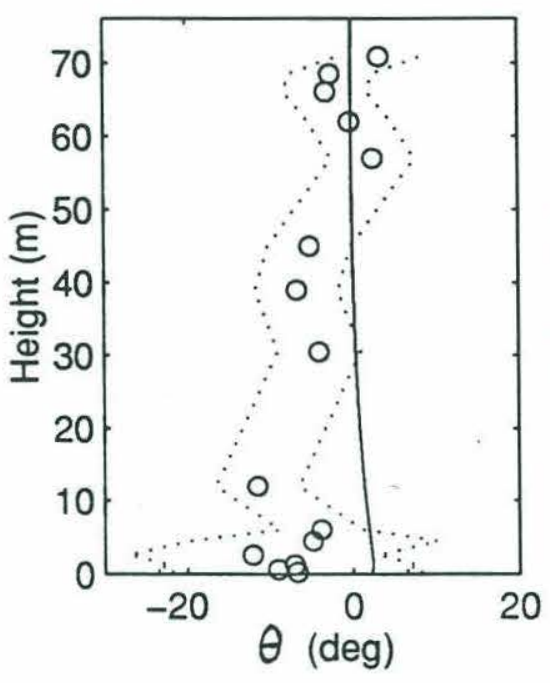

c)

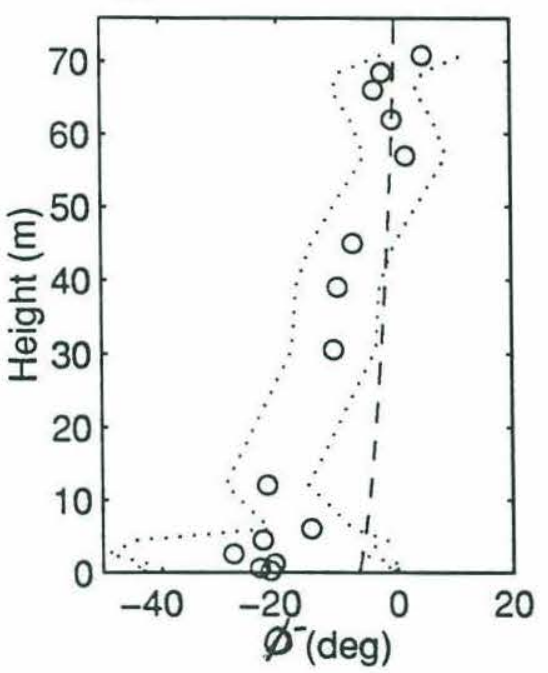

f)

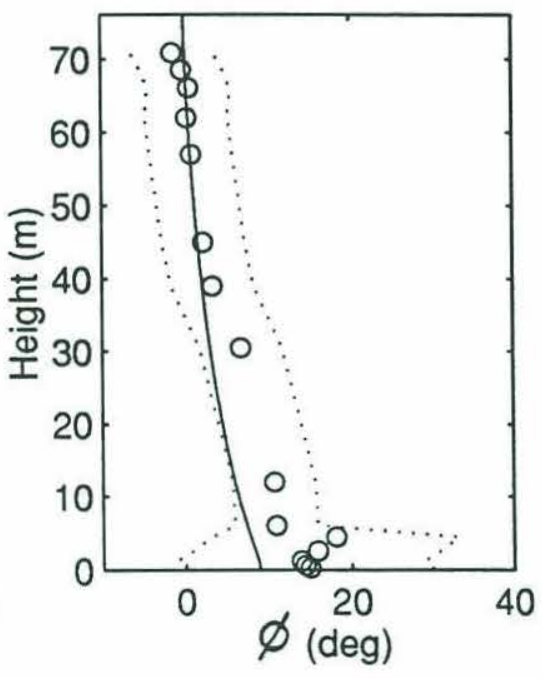

Figure 42: Model results for the vertical structure of the $M_{2}$ current ellipse obtained from the MY 2.5. Circles represent results obtained from BASS and VMCM measurements, and dotted lines depict the associated uncertainties (Figure 20). Profiles show the magnitudes of the rotary components $R^{+}$(solid line) and $R^{-}$(dashed line) (a), relative veering of the corresponding phase angles $\Delta \phi^{+}(\mathrm{c})$ and $\Delta \phi^{-}(\mathrm{d})$, current amplitudes $U_{M a j}$ and $U_{M i n}(\mathrm{~d})$, relative veering of the inclination of the major axis $\Delta \theta$ (e) and of the phase of the velocity vector $\Delta \phi(f)$. Decreasing angles $\Delta \theta$ and $\Delta \phi^{ \pm}$depict clockwise rotation of the major axis $U_{M a j}$ and rotary components $R^{ \pm}$, respectively. Decreasing angles $\Delta \phi$ represent phase lag. 


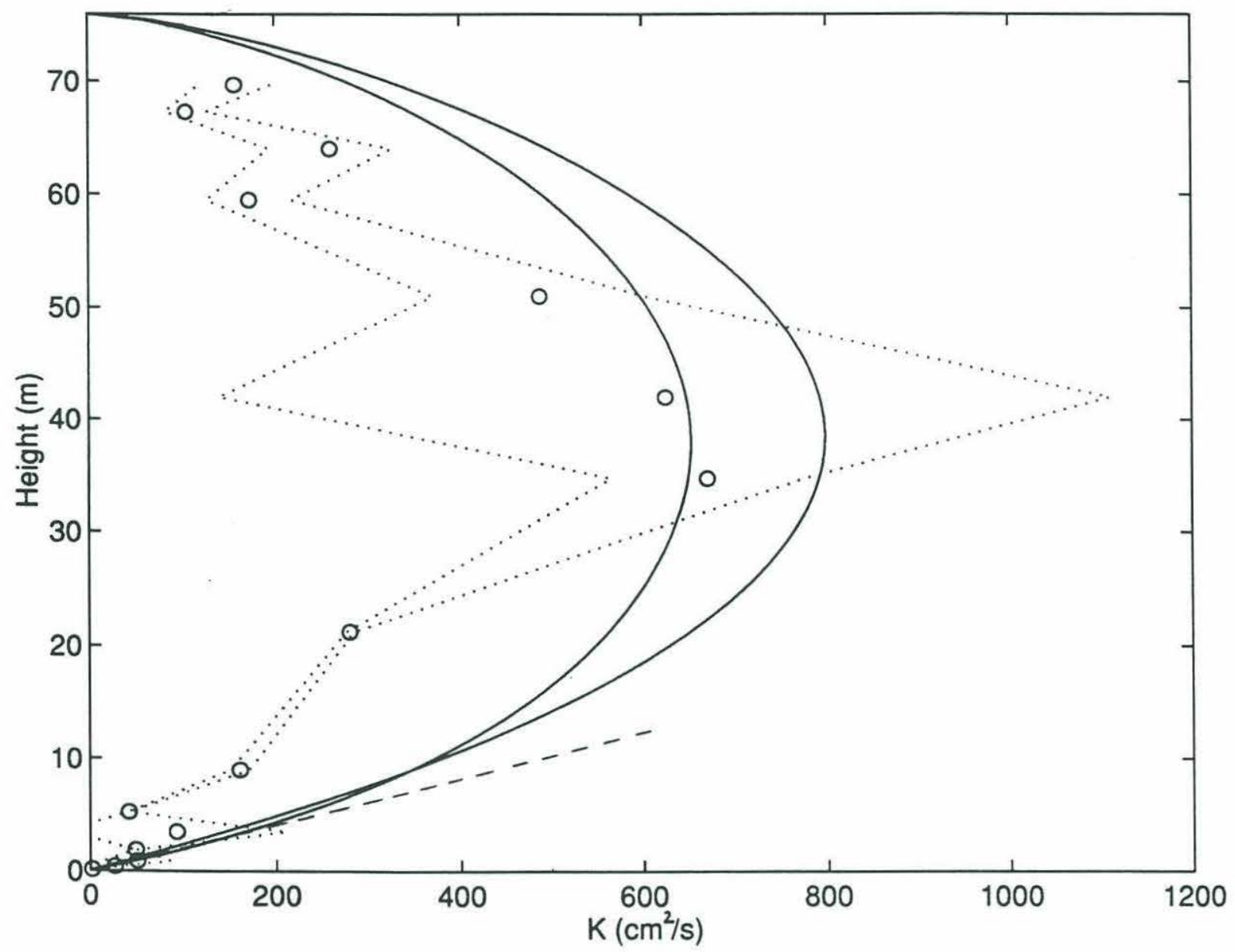

Figure 43: Numerical eddy viscocities $K$ obtained from the MY 2.5 according to their lowest and highest values during one tidal cycle. Circles represent results from measured data, and dotted lines are the corresponding uncertainties (Figure 28). The dashed line depicts the slope $\kappa \bar{u}_{*} z$, where $\bar{u}_{*}=1.27 \mathrm{~cm} / \mathrm{s}$ according to model results. 
a)

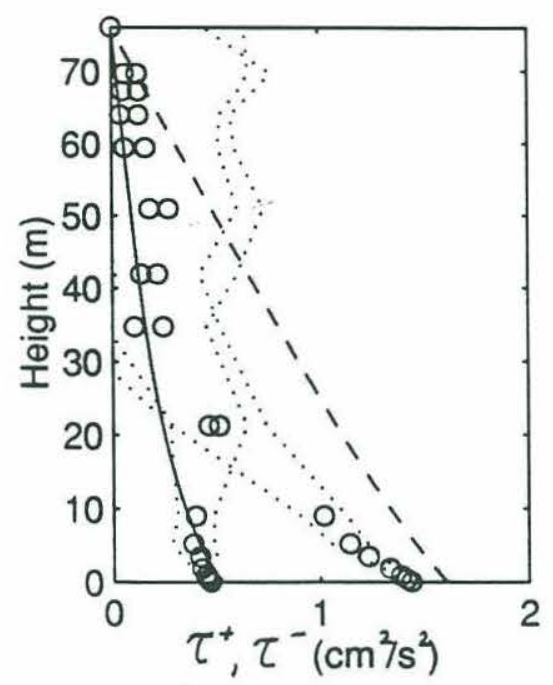

d)

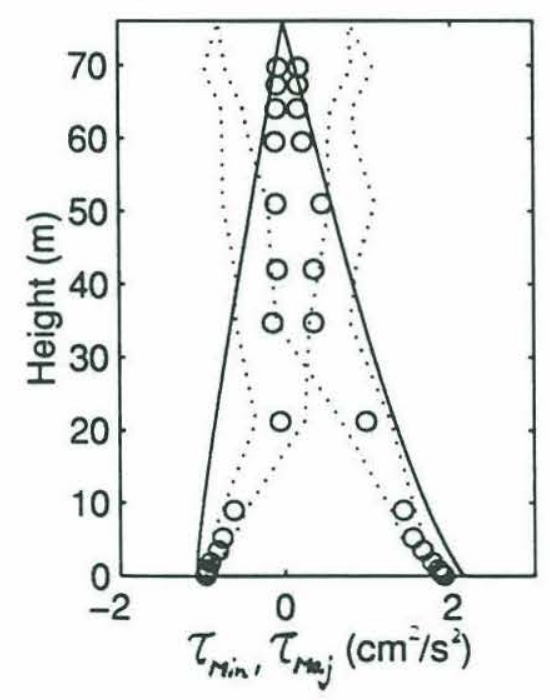

b)

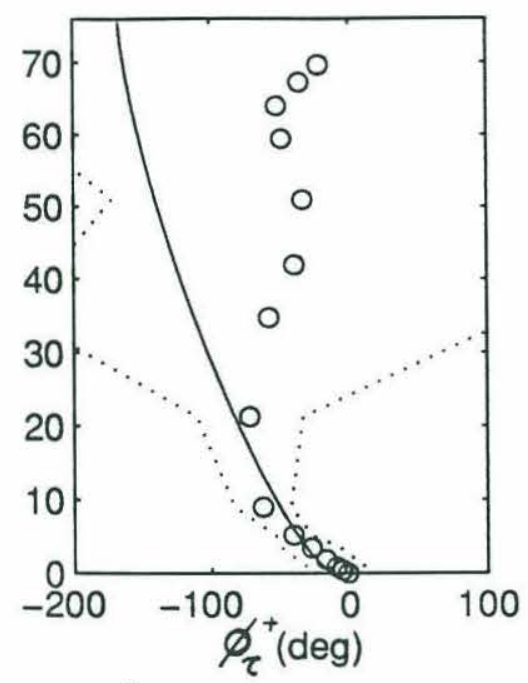

e)

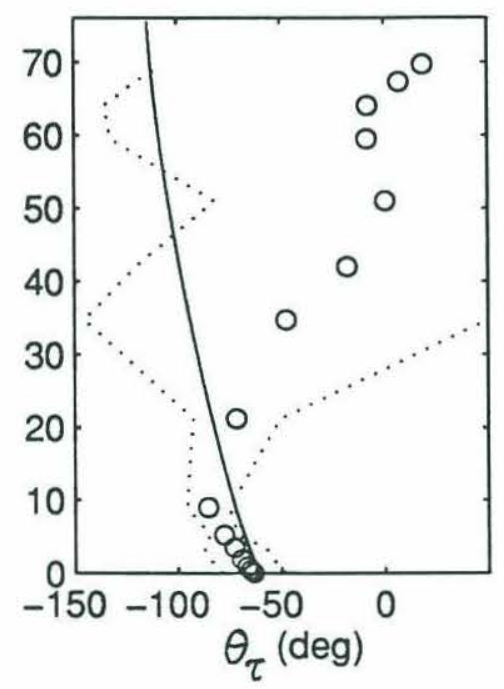

c)

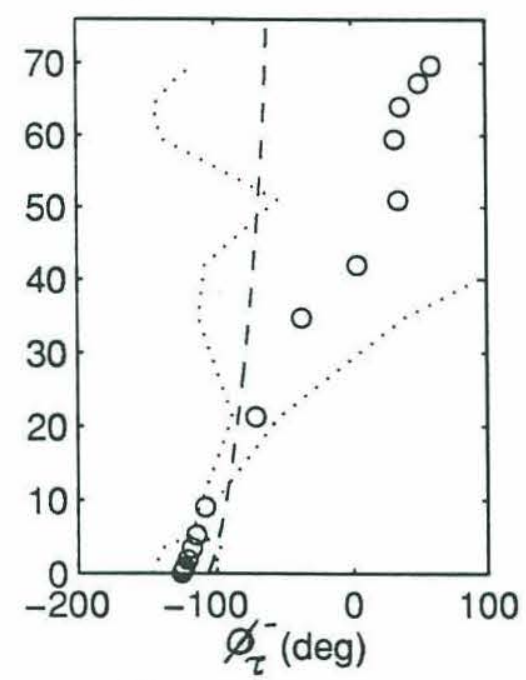

f)

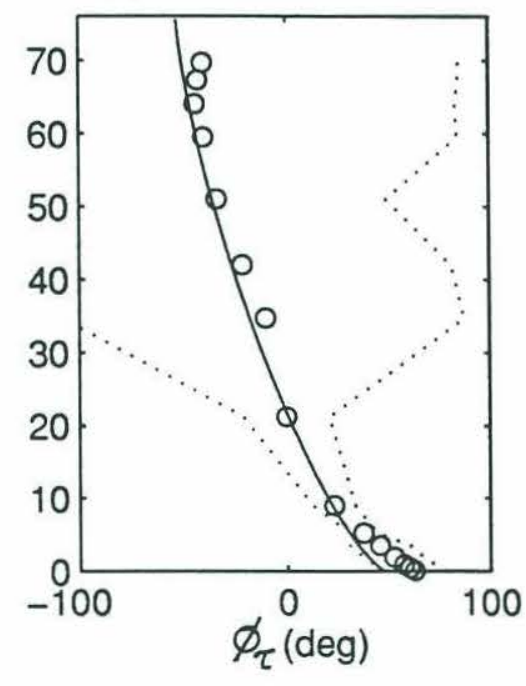

Figure 44: Model results for the vertical structure of the $M_{2}$ stress ellipse obtained from the MY 2.5. Circles represent results from actual data, and dotted lines are the associated uncertainties (Figure 25). Profiles show the magnitudes of the rotary components $\tau^{+}$(solid line) and $\tau^{-}$(dashed line) (a), corresponding phase angles $\phi_{\tau}^{+}(\mathrm{c})$ and $\phi_{\tau}^{-}(\mathrm{d})$, current amplitudes $\tau_{M a j}$ and $\tau_{M i n}(\mathrm{~d})$, inclination of the major axis $\theta_{\tau}(\mathrm{e})$, and phase of the stress vector $\phi_{\tau}(\mathrm{f})$. Inclination and phase angles of the rotary components are given with respect to east, where negative values are clockwise from east. Phase angles describe the orientation of the $M_{2}$ stress vector at the beginning of the time series. 
a)

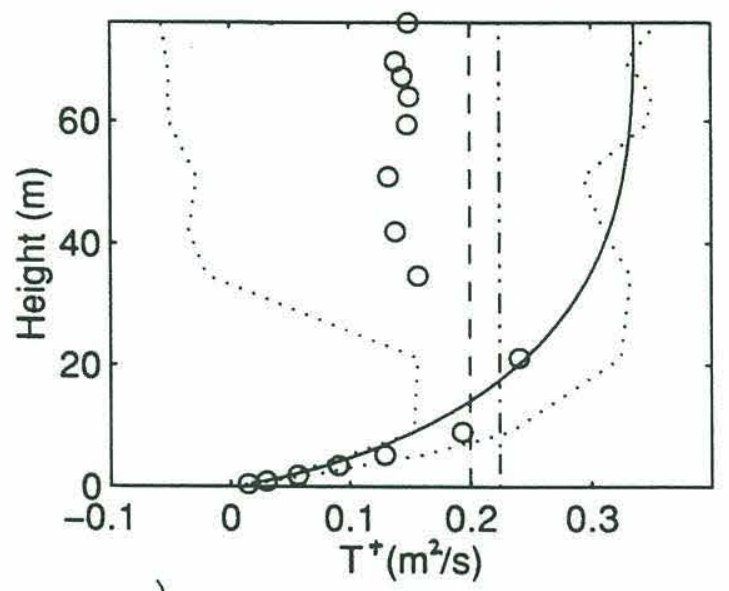

c)

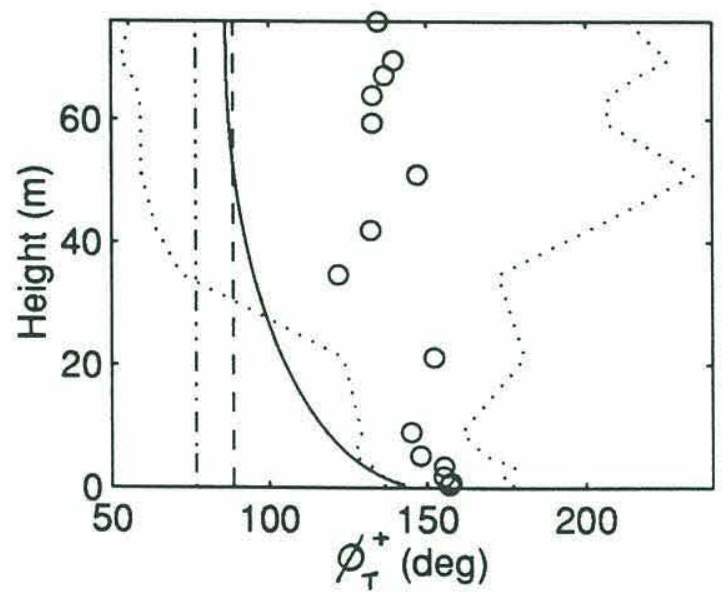

b)

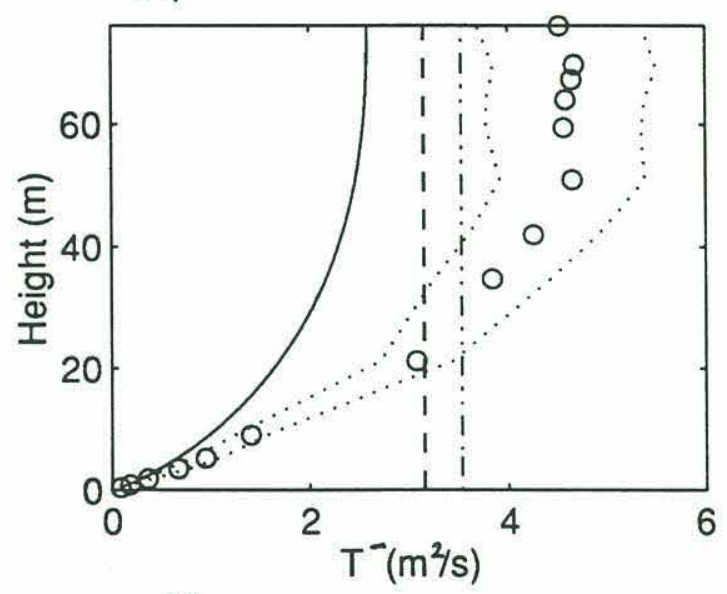

d)

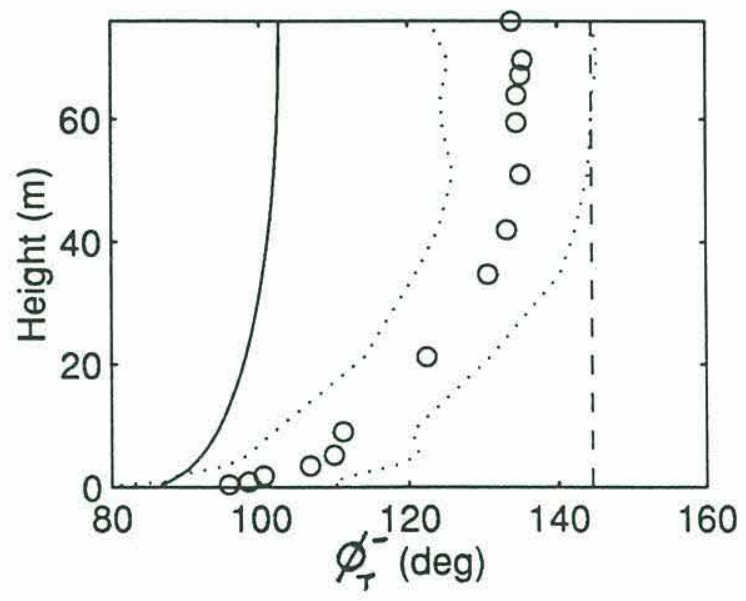

Figure 45: Model results for the integrated velocity defect of the $M_{2}$ constituent (solid lines), and tidal boundary layer transports predicted by numerical bottom stresses (dotdashed lines) obtained from the MY 2.5. Circles represent results from velocity defects of actual data, and dashed lines are boundary layer transports predicted by BASS bottom stress estimates (Figure 27). Dotted lines are uncertainties associated with the data. Profiles show transport magnitudes $T^{+}(\mathrm{a})$ and $T^{-}$(b), and corresponding phase angles $\phi_{T}^{+}$(c) and $\phi_{T}^{-}$(d). Phase angles describe the orientation of the $\mathrm{M}_{2}$ transport vector at the beginning of the time series and are given with respect to east, where negative values are clockwise from east. 


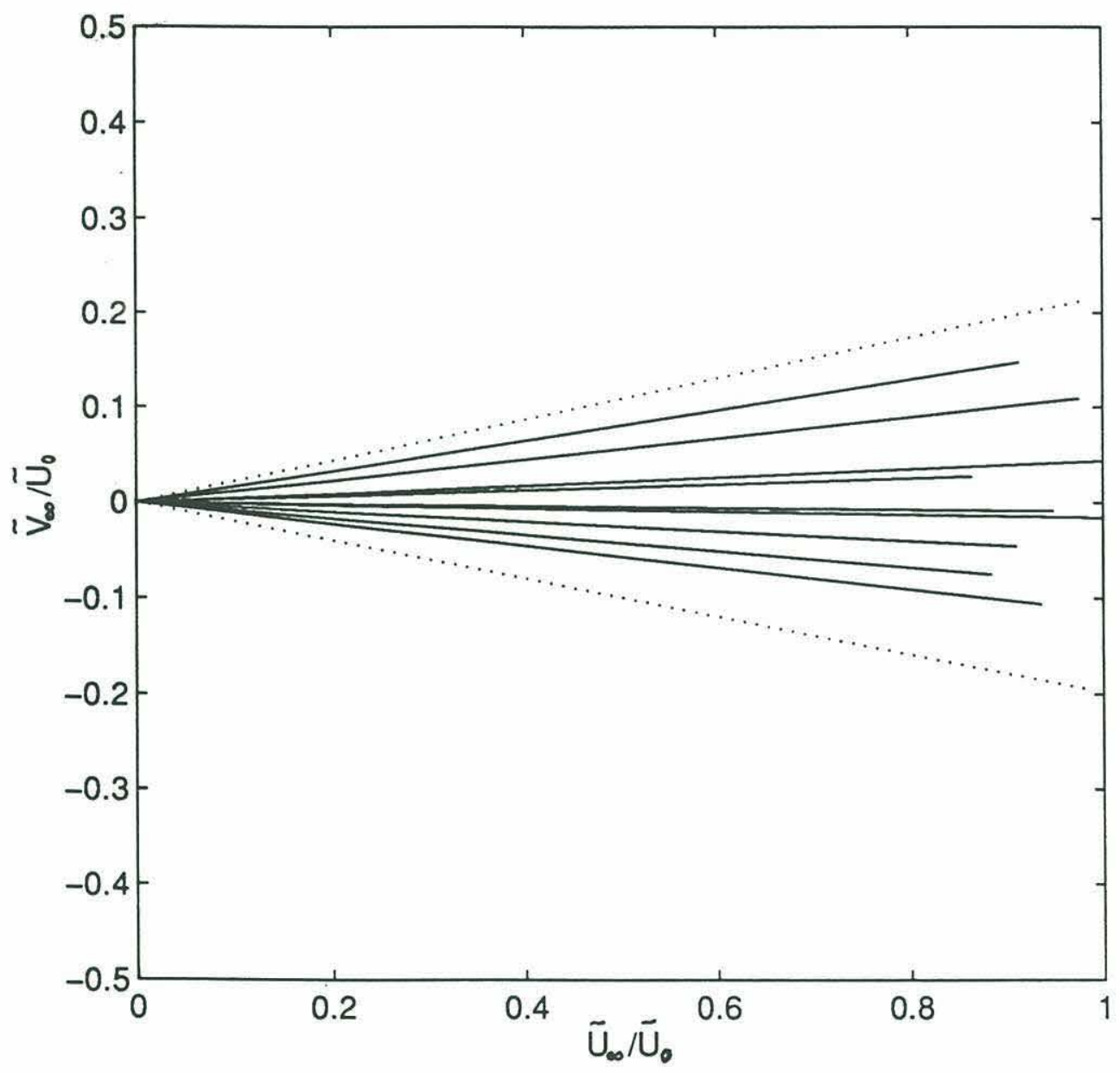

Figure 46: $\frac{\tilde{V}_{\infty}}{\tilde{U}_{0}}$ versus $\frac{\tilde{U}_{\infty}}{\tilde{U}_{0}}$, where $\tilde{U}_{\infty}$ and $\tilde{V}_{\infty}$ are characteristic interior subtidal velocities deduced from observations, and representative of times where acceleration of the mean flow as well as horizontal density gradients are small. $\tilde{U}_{0}$ is a characteristic free stream velocity computed under the assumption of barotropic, geostrophic flow, and is aligned with $\tilde{U}_{\infty} . \quad \tilde{V}_{0}$ is zero by definition. Dotted lines depict the outer margins of error bars. 
a)

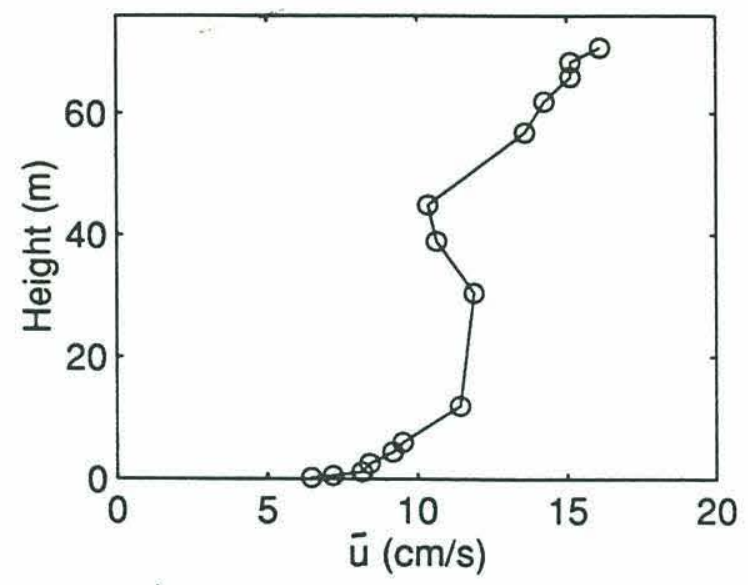

c)

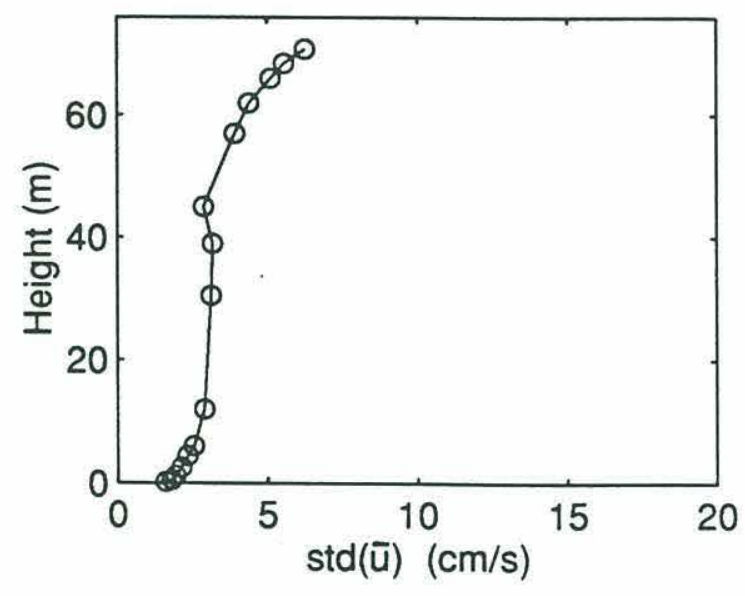

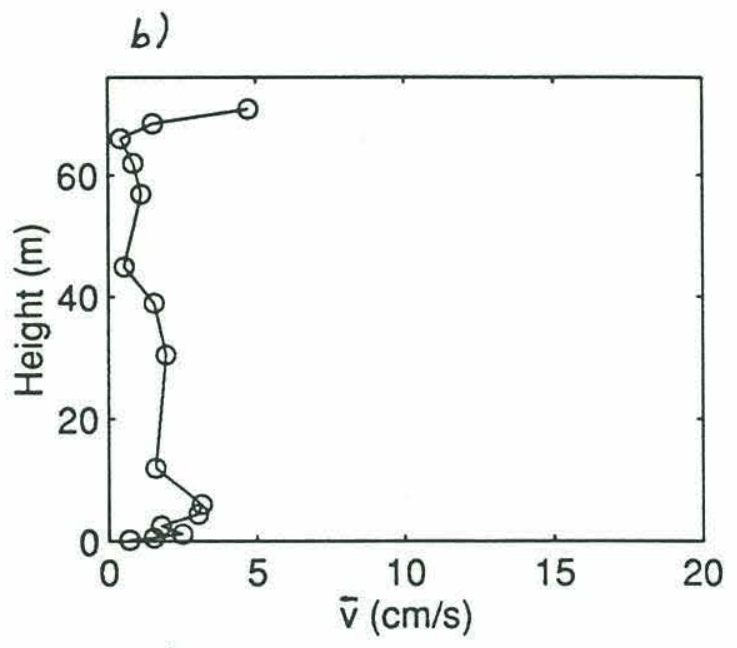

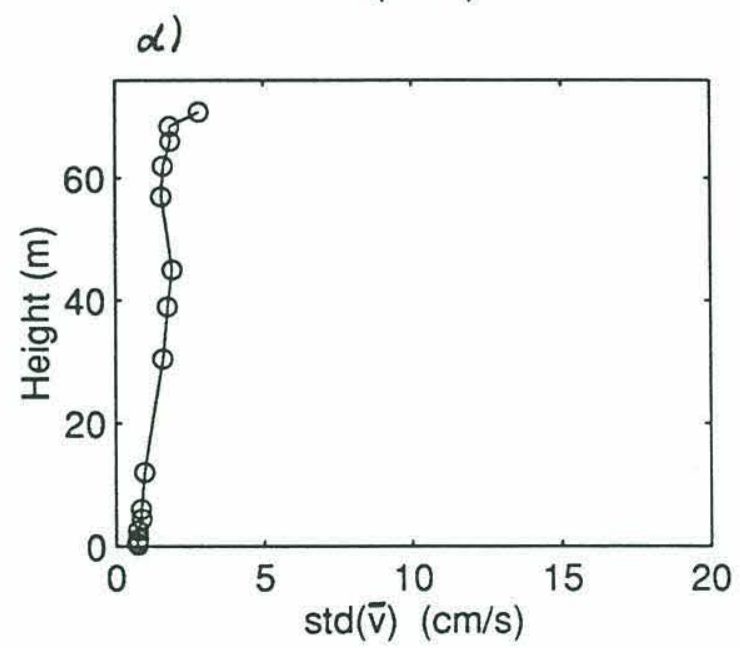

Figure 47: Velocity profiles of the subtidal current representative of times where acceleration of the mean flow and horizontal density gradients are small. The components $\bar{u}(\mathrm{a})$ and $\bar{v}(\mathrm{~b})$ are along-bank and cross-bank, respectively, where the along-bank direction was taken as $-149^{\circ}$ from east. Profiles are from averaged data, where the average was taken of all nine $12.42 \mathrm{hrs}$-bin events meeting the requirements of quasi-steadiness and small baroclinic pressure forcing. Standard deviations from the mean are shown (c) and (d). 
a)

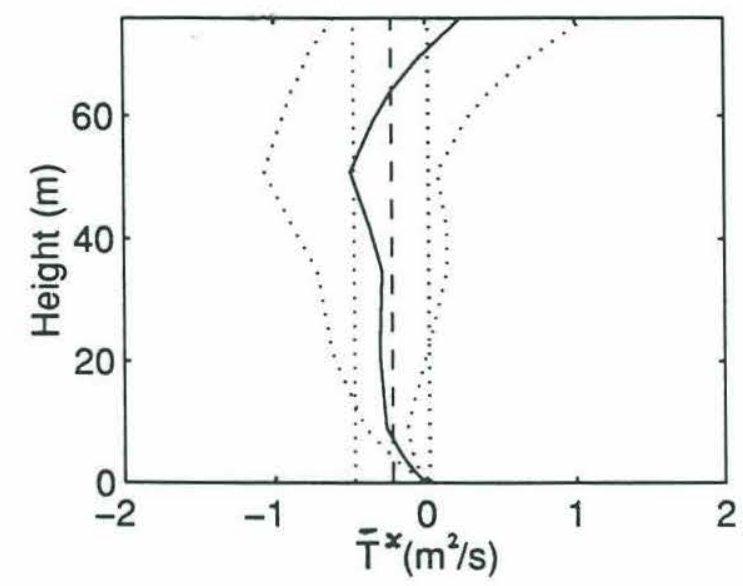

b)

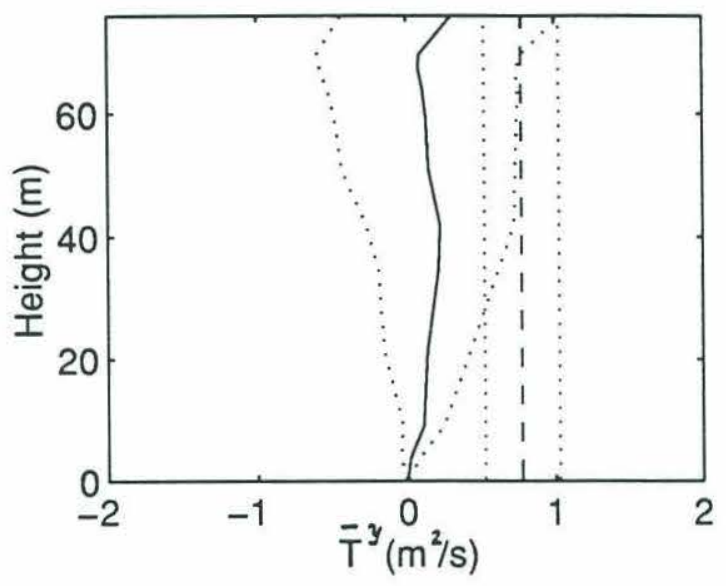

Figure 48: Integrated velocity defects $\bar{T}^{x}$ and $\bar{T}^{y}$ for the along-bank (a) and acrossbank (b) components of the subtidal flow (solid lines), and predicted boundary layer transports - $\frac{\bar{\tau}_{b}^{y}}{\rho_{0} f}$ and $\frac{\bar{\tau}_{b}^{g}}{\rho_{0} f}$ from low-pass filtered bottom stress estimates (dashed lines). Profiles are from averaged data, where the average was taken of all nine $12.42 \mathrm{hrs}$-bin events meeting the requirements of quasi-steadiness and small baroclinic pressure forcing. Dotted lines depict the standard deviation from the mean. 